\title{
Glucagon-Like Peptide-1 and Its Class B G Protein-Coupled Receptors: A Long March to Therapeutic Successes
}

Chris de Graaf, Dan Donnelly, Denise Wootten, Jesper Lau, Patrick M. Sexton, Laurence J. Miller, Jung-Mo Ahn, Jiayu Liao,

Madeleine M. Fletcher, Dehua Yang, Alastair J. H. Brown, Caihong Zhou, Jiejie Deng, and Ming-Wei Wang

Division of Medicinal Chemistry, Faculty of Sciences, Vrije Universiteit Amsterdam, Amsterdam, The Netherlands (C.d.G.); School of Biomedical Sciences, University of Leeds, Leeds, United Kingdom (D.D.); Drug Discovery Biology Theme and Department of Pharmacology, Monash Institute of Pharmaceutical Sciences, Parkville, Victoria, Australia (D.W., P.M.S., M.M.F.); Protein and Peptide Chemistry, Global Research, Novo Nordisk A/S, Måløv, Denmark (J.La.); Department of Molecular Pharmacology and Experimental Therapeutics, Mayo Clinic, Scottsdale, Arizona (L.J.M.); Department of Chemistry and Biochemistry, University of Texas at Dallas, Richardson, Texas (J.-M.A.); Department of Bioengineering, Bourns College of Engineering, University of California at Riverside, Riverside, California (J.Li.); National Center for Drug Screening and CAS Key Laboratory of Receptor Research, Shanghai Institute of Materia Medica, Chinese Academy of Sciences, Shanghai, China (D.Y., C.Z., J.D., M.-W.W.); Heptares Therapeutics, BioPark, Welwyn Garden City, United Kingdom (A.J.H.B.); and School of Pharmacy, Fudan University, Zhangjiang High-Tech Park, Shanghai, China (M.-W.W.)

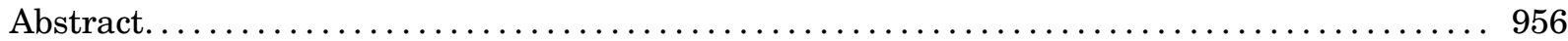

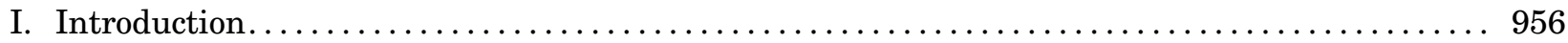

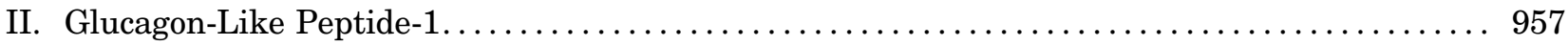

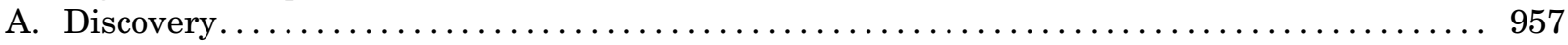

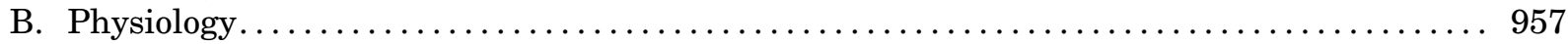

1. Effect on Glucose Homeostasis. ..................................... 958

2. Effect on Gastric Emptying. ..................................... 958

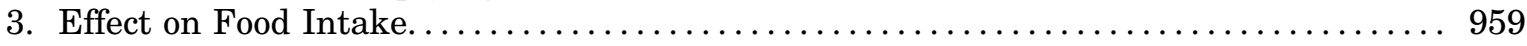

4. Effect on Cardiovascular Activity.................................... 959

5. Effect on Immune Response......................................... 959

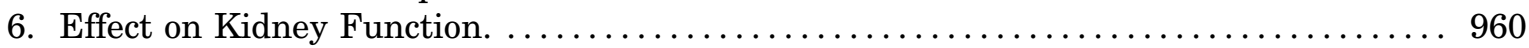

7. Effect on Nervous System......................................... 960

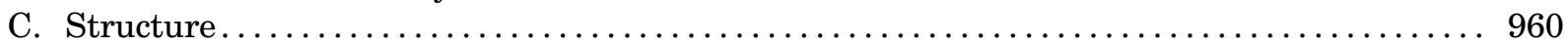

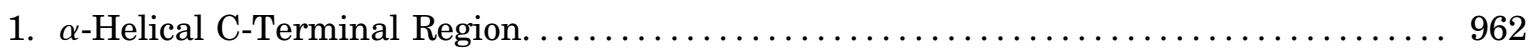

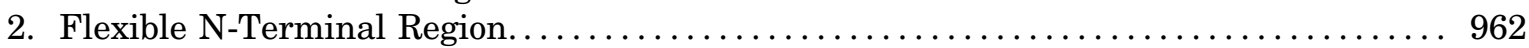

3. Interaction between the C-Terminal Helix and ECD . . . . . . . . . . . . . . . . . . . 963

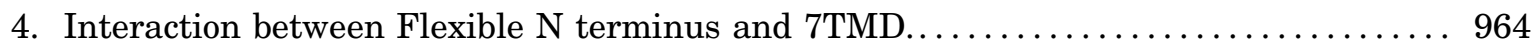

D. Mutagenesis ................................................... 964

1. Truncated GLP-1 Analogs........................................ 964

2. Chimeric GLP-1 Analogs. . . . . . . . . . . . . . . . . . . . . . . . . . . . . 965

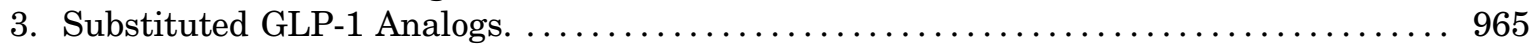

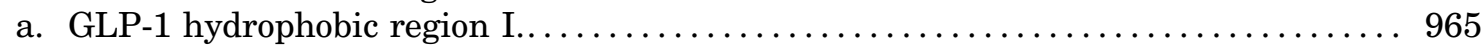

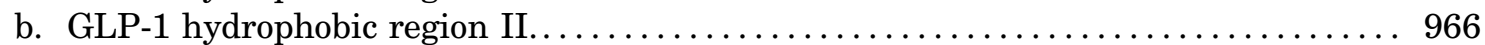

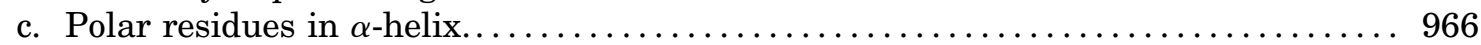

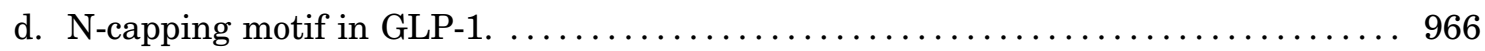

This work was supported by National Health and Family Planning Commission of China [2012ZX09304-011, 2013ZX09401003-005, 2013ZX09507001, and 2013ZX09507-002 to M.-W.W.]; Chinese Academy of Sciences [to M.-W.W.]; Shanghai Science and Technology Development Fund [15DZ2291600 to M.-W.W.]; Thousand Talents Program in China [to M.-W.W.]; the CAS-Novo Nordisk Research Fund [to D.Y.]; National Natural Science Foundation of China [81373463 to D.Y.]; National Health and Medical Research Council of Australia (NHMRC) Principal Research Fellowship [to P.M.S.]; NHMRC Program [Grant 1055134 to P.M.S.]; NHMRC Career Development Fellowship [to D.W.]; NHMRC Project [Grant 1061044 to P.M.S. and Grant 1065410 to D.W.]; Welch Foundation [AT-1595 to J.-M.A.]; and Juvenile Diabetes Research Foundation [37-2011-20 to J.-M.A.].

C.d.G., D.D., and D.W. contributed equally to this work.

Address correspondence to: Dr. Ming-Wei Wang, National Center for Drug Screening, Shanghai Institute of Materia Medica, Chinese Academy of Sciences, 189 Guo Shou Jing Road, Shanghai 201203, China. E-mail: mwwang@simm.ac.cn

dx.doi.org/10.1124/pr.115.011395. 


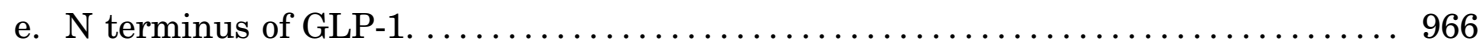

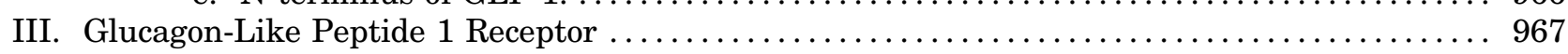

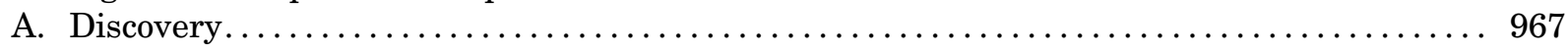

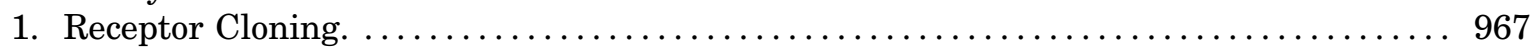

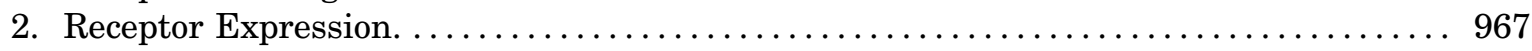

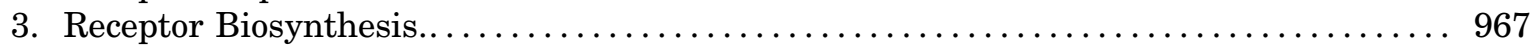

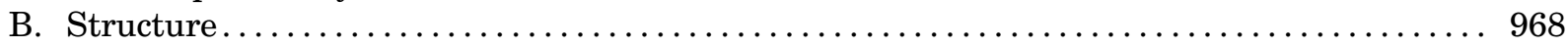

1. N-Terminal Domain. ............................................ 969

a. Structure determination of the N-terminal ECD..................... 969

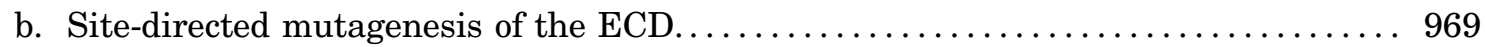

2. Seven-Transmembrane Domain. ................................. 969

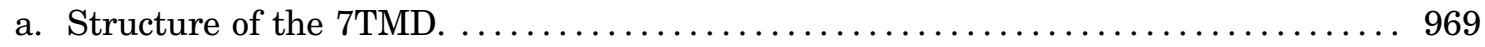

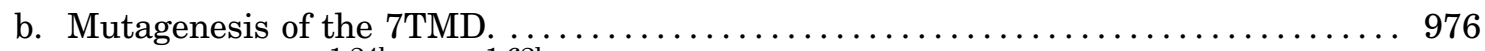

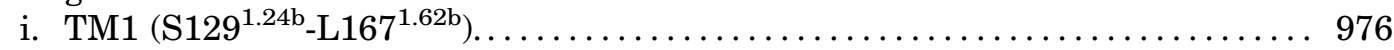

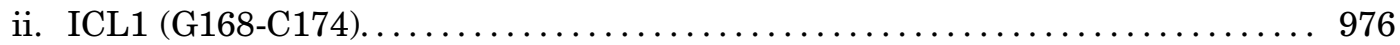

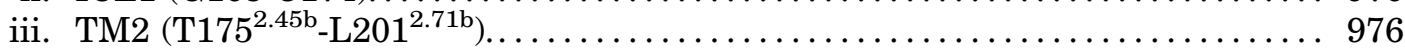

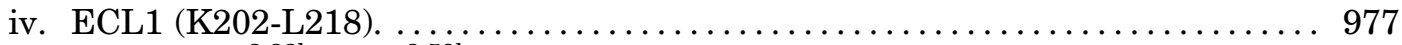

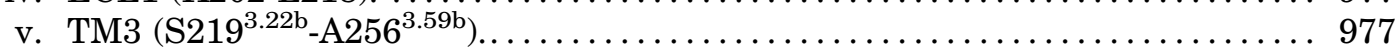

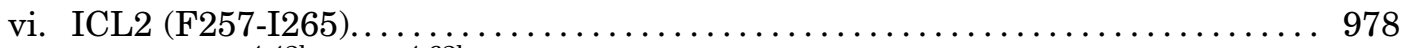

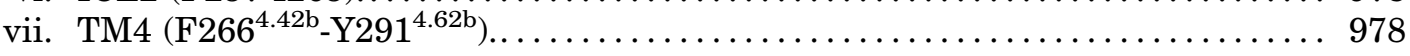

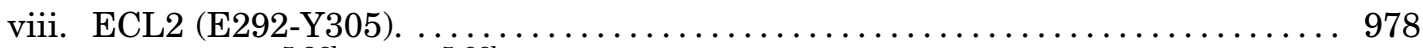

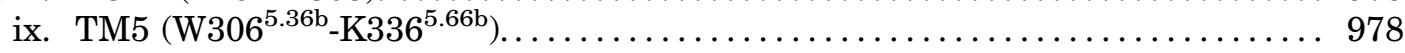

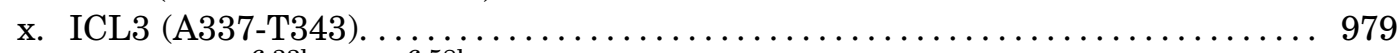

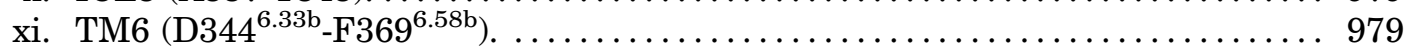

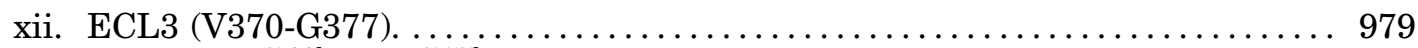

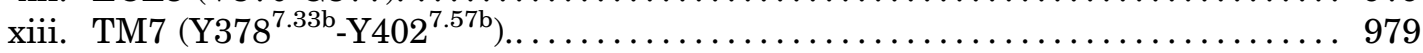

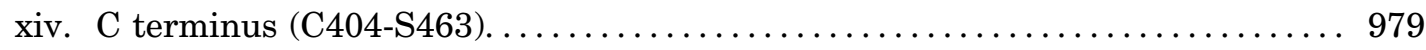

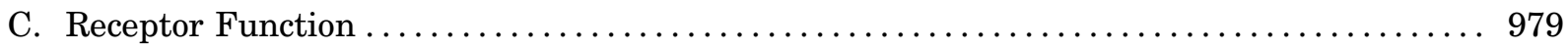

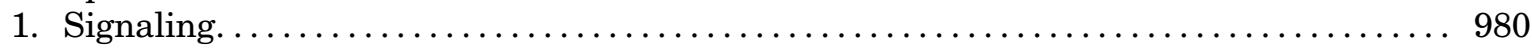

a. Recombinant cells. ........................................... 980

b. Pancreatic $\beta$ cells.............................................. 980

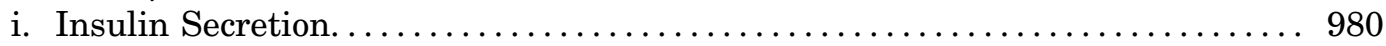

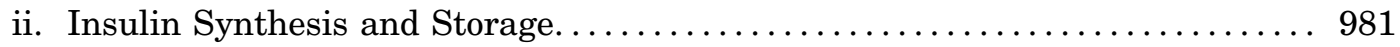

iii. $\beta$ Cell Survival, Proliferation, and Neogenesis..................... 981

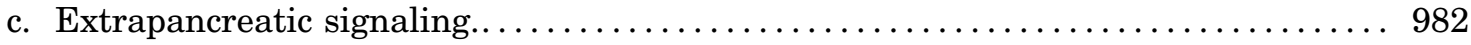

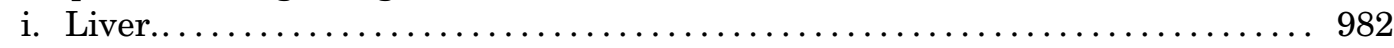

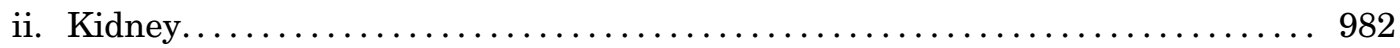

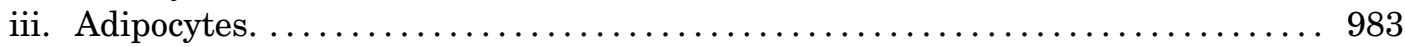

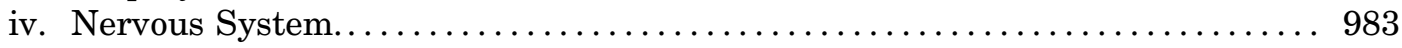

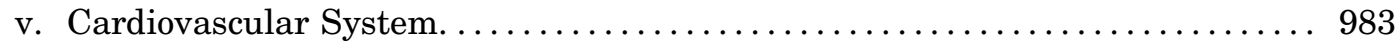

2. Ligand-Directed Signal Bias. .................................... 983

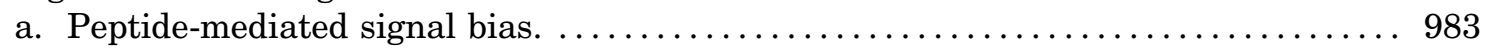

b. Nonpeptide-mediated bias. ................................. 984

ABBREVIATIONS: $\mathrm{A} \beta$, amyloid $\beta$ protein; $\mathrm{AD}$, Alzheimer's disease; Aib, aminoisobutyric acid; AMPK, AMP-activated protein kinase; $\mathrm{BCM}, \beta$ cell mass; BETP, 4-(3-benzyloxyphenyl)-2-ethylsulfinyl-6-(trifluoromethyl)pyrimidine; CD, circular dichroism; CHI, congenital hyperinsulinism; CHL, Chinese hamster lung; CHO, Chinese hamster ovary; CICR, calcium-induced calcium release; CNS, central nervous system; CREB, cAMP response element-binding protein; $\mathrm{CRF}_{1} \mathrm{R}$, corticotropin-releasing factor-1 receptor; $\mathrm{DPC}$, dodecylphosphocholine; DPP-4, dipeptidyl peptidase-4; ECD, extracellular domain; ECL, extracellular loop; $\mathrm{E}_{\max }$, maximal response; Epac, exchange protein activated by cAMP; ER, endoplasmic reticulum; ERK, extracellular regulated kinase; FDA, Food and Drug Administration; GCGR, glucagon receptor; GIP, gastric inhibitory polypeptide; GIPR, GIP receptor; GLP, glucagon-like peptide; GLP-1R, GLP-1 receptor; GPCR, G protein-coupled receptor; GRK, G proteincoupled receptor kinase; HbA1c, hemoglobin A1c; HEK, human embryonic kidney; hGLP-1R, human GLP-1R; iCa ${ }^{2+}$, intracellular calcium; ICL, intracellular loop; IP1, intervening peptide 1; IRS2, insulin receptor substrate 2; $\mathrm{K}_{\mathrm{ATP}}$, ATP-sensitive potassium; MAPK, mitogen-activated protein kinase; NEP 24.11, neutral endopeptidase 24.11; NHE3, $\mathrm{Na}^{+} / \mathrm{H}^{+}$exchanger isoform 3; PACAP, pituitary adenylate cyclase-activating polypeptide; PD, Parkinson's disease; PDB, Protein Data Bank; PDX-1, pancreatic and duodenal homeobox-1; PET, positron emission tomography; PI3K, phosphatidylinositol 3-kinase; PKA, protein kinase A; PKB, protein kinase B; PKC, protein kinase C; PMA, phorbol myristate acetate; PPAR, peroxisome proliferator-activated receptor; Rim, Rab-3-interacting molecule; SAR, structure-activity relationship; SNP, single-nucleotide polymorphism; SPECT, single-photon emission-computed tomography; SRP, signal recognition particle; SUR1, sulfonylurea receptor; T2DM, type 2 diabetes mellitus; TM, transmembrane; 7TM, seven transmembrane; 7TMD, 7TM domain. 


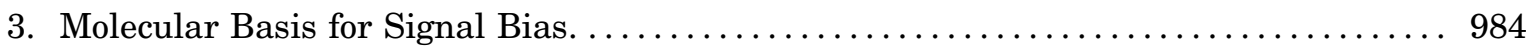

4. Allosteric Modulation. ............................................. 985

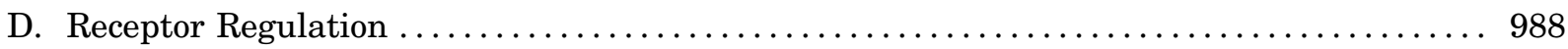

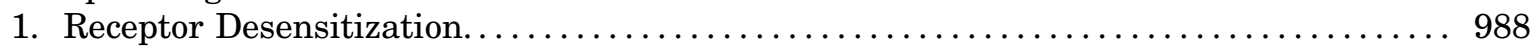

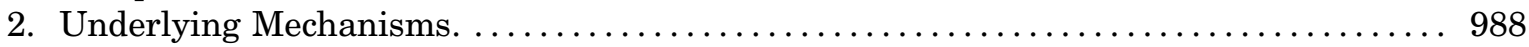

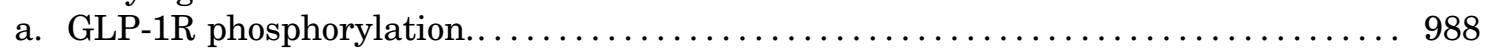

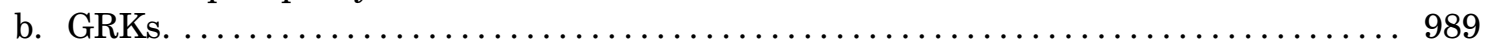

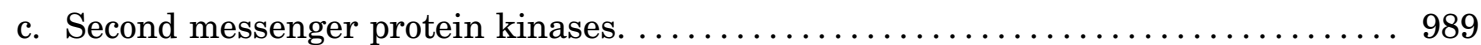

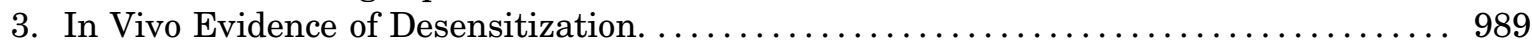

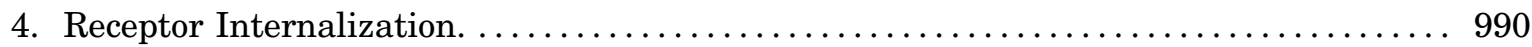

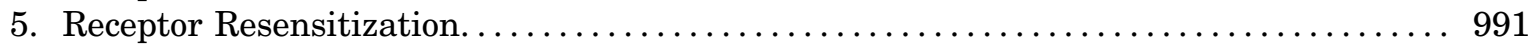

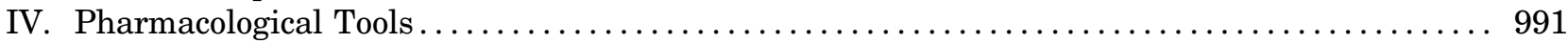

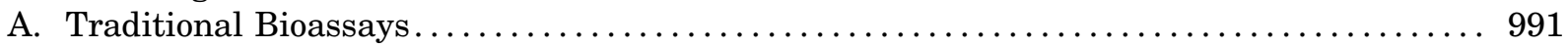

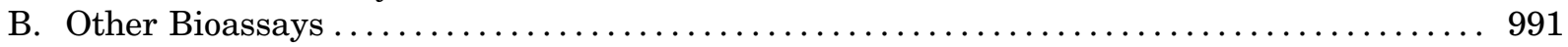

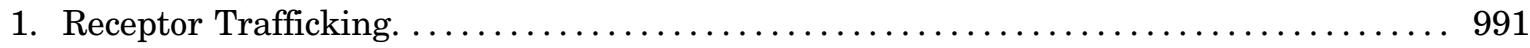

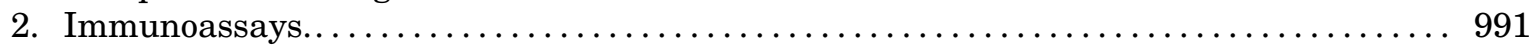

3. Pancreatic $\beta$ Cell Regeneration................................... 992

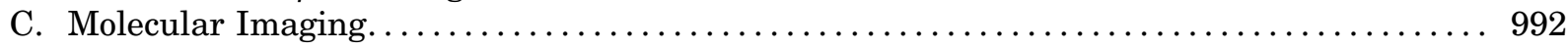

V. Pharmaceutical Development and Therapeutics ............................. 994

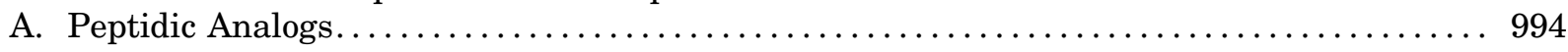

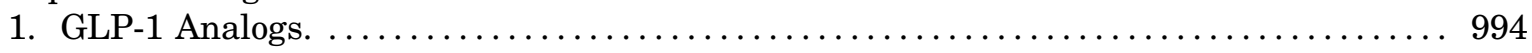

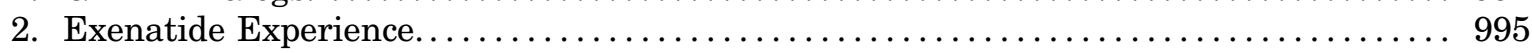

3. GLP-1 Mimetics. ........................................... 996

B. Nonpeptidic Modulators ...................................... 996

C. Currently Approved Clinical Applications .............................. 998

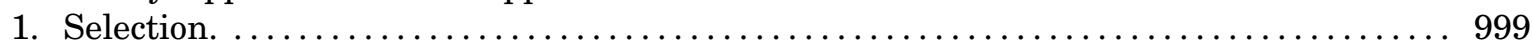

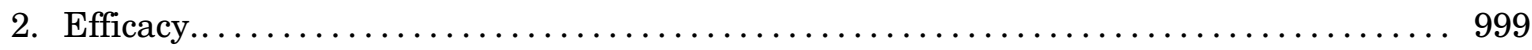

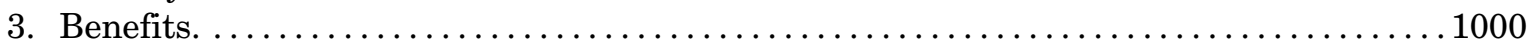

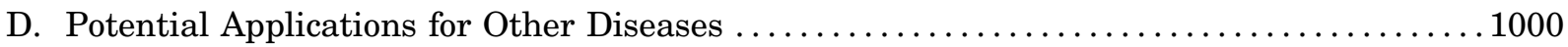

1. Neurologic Disorders............................................... 1000

2. Oncological Association. ........................................... 1001

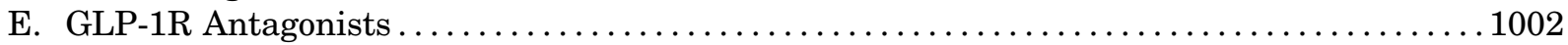

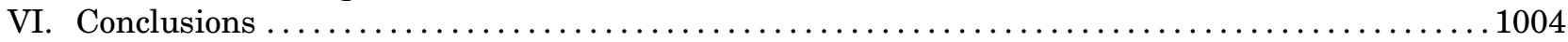

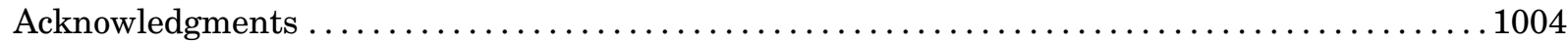

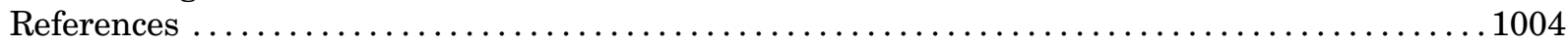

\begin{abstract}
The glucagon-like peptide (GLP)-1 receptor (GLP-1R) is a class B G protein-coupled receptor (GPCR) that mediates the action of GLP-1, a peptide hormone secreted from three major tissues in humans, enteroendocrine $L$ cells in the distal intestine, $\alpha$ cells in the pancreas, and the central nervous system, which exerts important actions useful in the management of type 2 diabetes mellitus and obesity, including glucose homeostasis and regulation of gastric motility and food intake. Peptidic analogs of GLP-1 have been successfully developed with enhanced bioavailability and pharmacological activity. Physiologic and biochemical studies with truncated, chimeric, and mutated peptides and GLP-1R variants, together with ligand-bound crystal structures of the extracellular domain and the first three-dimensional

structures of the 7-helical transmembrane domain of class B GPCRs, have provided the basis for a twodomain-binding mechanism of GLP-1 with its cognate receptor. Although efforts in discovering therapeutically viable nonpeptidic GLP-1R agonists have been hampered, small-molecule modulators offer complementary chemical tools to peptide analogs to investigate ligand-directed biased cellular signaling of GLP-1R. The integrated pharmacological and structural information of different GLP-1 analogs and homologous receptors give new insights into the molecular determinants of GLP-1R ligand selectivity and functional activity, thereby providing novel opportunities in the design and development of more efficacious agents to treat metabolic disorders.
\end{abstract}

\section{Introduction}

Glucagon-like peptide (GLP)-1 is a gastrointestinal peptide hormone secreted from three major tissues in humans, enteroendocrine L cells in the distal intestine, $\alpha$ cells in the pancreas, and the central nervous system, which has multiple therapeutic effects useful in the management of type 2 diabetes mellitus (T2DM). These include most prominently a glucose-dependent insulinotropic function and other actions on glucose homeostasis, as well as benefits to gastric emptying and appetite regulation 
valuable in reducing food intake and body weight. This hormone exerts its effects by binding to and activating a class B G protein-coupled receptor (GPCR), namely, GLP-1 receptor (GLP-1R). We review the current understanding of the structures of GLP-1 and GLP-1R, the molecular basis of their interaction, and the signaling events associated with it. We also discuss the peptide analogs and nonpeptidic ligands that have been developed to target GLP1R, the molecular basis of their action, and the implications for ligand-biased activity and allosteric regulation of this hormone-receptor system. Some of these GLP-1R agonists are already in clinical use, with many more currently being developed, and likely to provide enhancements in their ease of administration, tolerability, and effectiveness.

\section{Glucagon-Like Peptide-1}

\section{A. Discovery}

GLP-1 is a member of the incretin family of gastrointestinal hormones (Creutzfeldt, 1979; Baggio and Drucker, 2007; Campbell and Drucker, 2013; Heppner and PerezTilve, 2015). In 1906, Moore and his colleagues tested the hypothesis that the pancreas might be stimulated by factors from the gut to help disposal of nutrients and started using porcine small intestine extract to treat diabetic patients (Moore, 1906). In 1928, Zunc and LaBarre were able to show a hypoglycemic effect following injection of secretin extracted from the small intestinal mucosa, and this effect was mediated through the pancreas (Zunz and LaBarre, 1928). Subsequently, the term incrétine (incretin) was introduced by LaBarre for a substance extracted from the upper gut mucosa, which produces hypoglycemia, but does not stimulate pancreatic exocrine secretion (LaBarre, 1932). It was later observed that orally administered glucose evoked a much stronger insulin release than that induced by i.v. injected glucose, supporting the concept of an entero-insular axis, that is, gut factor-stimulated insulin secretion (Elrick et al., 1964; McIntyre et al., 1964; Perley and Kipnis, 1967). The first discovered incretin hormone was gastric inhibitory polypeptide (GIP), which was isolated from crude extracts of the porcine small intestine for its activity to inhibit gastric acid secretion (Brown et al., 1975). This was followed by the observation that GIP could also stimulate insulin secretion in animals and humans, and thus, it was later renamed as glucose-dependent insulinotropic polypeptide, while retaining the same acronym (Dupre et al., 1973; Elahi et al., 1979; Sarson et al., 1984; Creutzfeldt and Ebert, 1985). GIP is released from the K cells of the small intestine. However, antibodies raised against GIP did not abolish the incretin effect, implying the existence of other prominent gut insulinotropic factors (Ebert and Creutzfeldt, 1982).

In 1981, GLP-1, the second incretin hormone, was identified in the translational products of mRNAs isolated from the pancreatic islets of anglerfish (Lund et al., 1981; Shields et al., 1981). Subsequently, both
GLP-1 and GLP-2 were confirmed from cloned hamster and human preproglucagon cDNAs, but only GLP-1 was able to stimulate insulin secretion (Bell et al., 1983a, 1983b; Mojsov et al., 1987). The proglucagon gene is expressed in the $\alpha$ cells of the pancreas, the L cells of the intestine, and neurons in the caudal brainstem and hypothalamus (Mojsov et al., 1986; Drucker and Asa, 1988) (Fig. 1). Although its transcription produces the same single mRNA in these cell types, the 180-residue preproglucagon protein translated from it is cleaved differently in the pancreas than in the intestine (and brain) by differential posttranslational processing: the former releases glicentin-related pancreatic peptide, glucagon, intervening peptide 1 (IP1), and major proglucagon fragment (containing GLP-1, IP1, and GLP-2 as a single fusion peptide), whereas the latter releases glicentin, oxyntomodulin, GLP-1, IP1, and GLP-2 (Mojsov et al., 1986) (Fig. 1). Endogenous GLP-1 exists in two forms: one corresponds to proglucagon 78-107 with its C-terminal Arg amidated, that is, GLP-1 $1_{7-36}$ amide, and the other is longer and not amidated, GLP- $1_{7-37}$ (Holst et al., 1987; Orskov et al., 1989). Both have similar biologic activities, although the amide form may have slightly improved stability in the circulation (Wettergren et al., 1998). GLP-1, but not GLP-2, was later demonstrated to enhance glucose-stimulated insulin secretion in response to nutrient ingestion (Schmidt et al., 1985; Kreymann et al., 1987; Mojsov et al., 1987; Orskov et al., 1987).

The half-life of GLP-1 peptide in the circulation is very short (less than 2 minutes). Its rapid inactivation is mainly due to the cleavage of two amino acids from the $\mathrm{N}$ terminus by the ubiquitous proteolytic enzyme dipeptidyl peptidase-4 (DPP-4) (Deacon et al., 1995b) (Fig. 1). In addition, a membrane-bound zinc metallopeptidase, neutral endopeptidase 24.11 (NEP 24.11), has also been shown to cleave GLP-1 at its C terminus both in vitro and in vivo (Plamboeck et al., 2005). The metabolites of GLP-1 are then subject to renal clearance by several mechanisms (Ruiz-Grande et al., 1993).

\section{B. Physiology}

The main action of GLP-1 is to work as an incretin, that is, as a gut-derived hormone capable of potentiating insulin secretion in the presence of high plasma glucose levels. The incretin concept came from observations that insulin release was higher when the glucose was administered orally rather than i.v., even when the same plasma glucose concentration was achieved from both routes (McIntyre et al., 1964; Perley and Kipnis, 1967). Although many hormones were originally suspected to contribute to the incretin effect, the current view is that GLP-1 and GIP are responsible for most incretin activity normally observed (Holst and Orskov, 2001; Vilsboll and Holst, 2004; Creutzfeldt, 2005; Campbell and Drucker, 2013). Oral administration of glucose results in a two- to threefold greater insulin secretion than i.v. glucose. Both GLP-1 and GIP can 


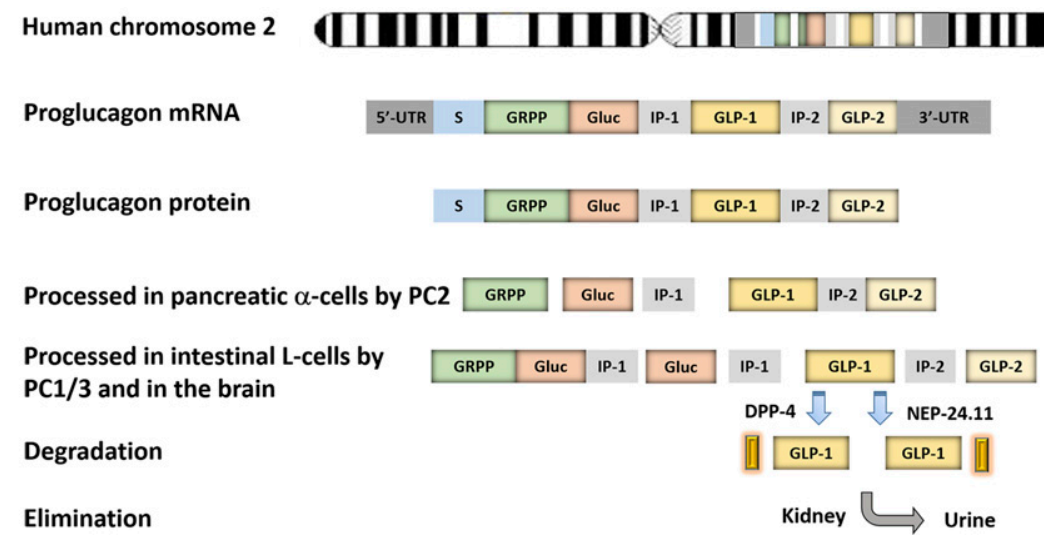

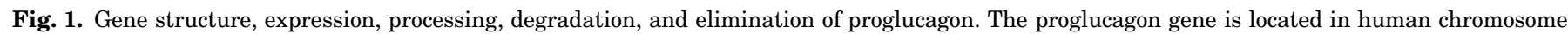

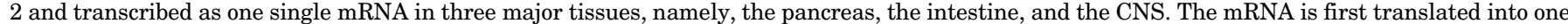

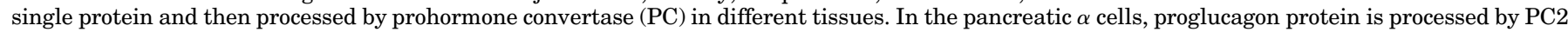

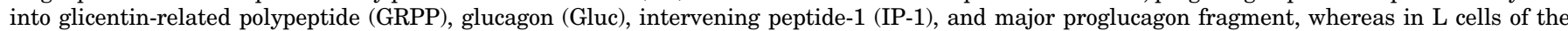

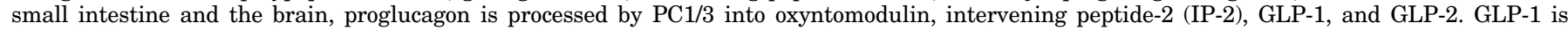

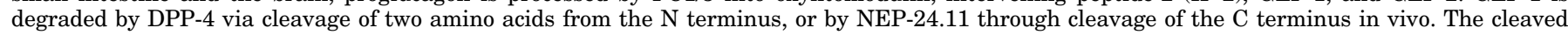
products are eventually eliminated in the kidney. UTR, untranslated region.

enhance insulin secretion after a mixed meal, but GLP1 is more potent than GIP (Nauck et al., 1993b; Elahi et al., 1994). In the human circulation, GIP concentration is eightfold higher than GLP-1; in type 2 diabetic patients, GLP-1 has more activity than GIP, but their effects on insulin secretion seem to be additive (Nauck et al., 1993a, 2004; Elahi et al., 1994).

1. Effect on Glucose Homeostasis. The insulinotropic activity of GLP-1 is strictly glucose-dependent mediated through its receptor at the membrane of pancreatic $\beta$ cells (Kreymann et al., 1987; Mojsov et al., 1987; Holst, 2007). GLP-1 could not stimulate insulin secretion at low levels of glucose in humans (Kreymann et al., 1987). GLP-1R belongs to the class B GPCR subfamily whose members include receptors for peptidic hormones such as glucagon, secretin, GIP, etc. The binding of GLP-1 to its receptor activates heterotrimeric Gs protein, which subsequently elicits adenylate cyclase activity, resulting in cAMP formation (Gromada et al., 1998, 2004). The increased level of cAMP in turn leads to activation of protein kinase $\mathrm{A}$ (PKA) and the cAMP-regulated guanine nucleotide exchange factor II (Ozaki et al., 2000). In the presence of high levels of glucose, GLP-1 has an effect on ATP-sensitive $\left(\mathrm{K}_{\mathrm{ATP}}\right)$ or voltage-gated potassium and calcium channels, resulting in membrane depolarization and $\mathrm{Ca}^{2+}$ release from both internal and extracellular stores. Increased $\mathrm{Ca}^{2+}$ together with cAMP will then promote exocytosis of vesicles containing insulin (Prentki and Matschinsky, 1987; Renstrom et al., 1997). This glucosedependent insulinotropic action of GLP-1 involves the glucose transporter, metabolic $\mathrm{ADP} / \mathrm{ATP}$ ratio, $\mathrm{K}_{\mathrm{ATP}}$ inhibition, $\mathrm{Ca}^{2+}$ channel opening, and, ultimately, insulin secretion (Gromada et al., 2004; Dyachok et al., 2006).

Another major activity of GLP-1 to reduce blood glucose relates to the suppression of glucagon secretion from $\alpha$ cells of the endocrine pancreas (Gromada and Rorsman, 2004). Glucagon is a major hyperglycemic hormone, in addition to epinephrine, and its release is reciprocally correlated with insulin secretion in glucose oscillation to mobilize hepatic glucose in the fasting state, thereby helping to ensure the maintenance of normoglycemia. In T2DM, both fasting hyperglucagonemia and exaggerated glucagon responses, which most likely contribute to the hyperglycemia of patients, were observed (Shah et al., 2000; Toft-Nielsen et al., 2001). Interestingly, only GLP-1, but not GIP, inhibits glucagon secretion (Nauck et al., 1993b). However, the detailed mechanism(s) by which GLP-1 suppresses glucagon secretion remains unclear. Because the levels of GLP-1 mRNA detected in $\alpha$ cells varied between none and $20 \%$ of a cell population, it is generally thought that local elevated insulin and somatostatin in response to GLP-1 stimulation are capable of suppressing glucagon secretion in $\alpha$ cells (Orskov et al., 1988; Heller et al., 1997; de Heer et al., 2008; Godoy-Matos, 2014). Nonetheless, in type 1 diabetic patients with absent $\beta$ cell activity who lack insulin and somatostatin, GLP-1 could still reduce glucose concentrations, suggesting a direct suppression of glucagon secretion (Creutzfeldt et al., 1996; Gromada and Rorsman, 2004).

2. Effect on Gastric Emptying. GLP-1 also has an important inhibitory activity on gut motility and gastrointestinal secretion (Wettergren et al., 1993; Nauck et al., 1997). It not only inhibits meal-induced pancreatic secretion, but also the gastric emptying process in humans (Wettergren et al., 1993). Its suppression of gastrin-induced acid secretion was demonstrated by injection of GLP-1 and/or peptide YY. Both peptides are released from $L$ cells in the ileal mucosa of healthy people (Wettergren et al., 1997) and can exhibit additive effects on gastrin-stimulated acid secretion, a function of unabsorbed nutrients in the ileum (Holst, 1997). It was subsequently shown that this inhibitory action of GLP-1 is mediated via a vagal pathway (Wettergren et al., 1994). This ileal-brake activity of GLP-1 was 
further demonstrated using the GLP-1R antagonist exendin $_{9-39}$, and therefore, is believed to have physiologic relevance (Schirra et al., 2006; Maljaars et al., 2008).

3. Effect on Food Intake. Another physiologic function of GLP-1 concerns inhibition of food intake that may have therapeutic value for body weight reduction. Whether this is related to its ileal brake effect is still debated. At least two neural mechanisms, central and peripheral, are involved in GLP-1 suppression of appetite and food intake. GLP-1 is expressed in the neurons of the brain stem, and GLP-1R is present in the hypothalamic areas that control energy homeostasis and food intake, including the arcuate nucleus, paraventricular nucleus, and dorsomedial nucleus (Jin et al., 1988; Kanse et al., 1988; Zheng et al., 2015). Intracerebroventricular injection of GLP-1 inhibits food intake in rats, and this activity is blocked by exendin ${ }_{9-39}$ (Tang-Christensen et al., 1996; Turton et al., 1996), or by the arcuate nucleus-damaging reagent, monosodium glutamate (Tang-Christensen et al., 1998). In contrast, GLP-1 released by L cells of the intestine after a meal inhibits gut mobility and gastric emptying, allowing nutrients in the ileum to reduce food intake (Read et al., 1994). Indeed, infusion of GLP-1 into normal human subjects significantly enhances satiety and decreases food intake (Flint et al., 1998). Consistent findings have shown that GLP-1R agonism promotes weight loss and improves glucose homeostasis in rodents, monkeys, and humans (Verdich et al., 2001; Barrera et al., 2011), and such weight-reducing properties have also been well-documented for two marketed GLP-1 mimetics, exenatide (exendin-4) and liraglutide (Moretto et al., 2008; Astrup et al., 2009; Norris et al., 2009; Lean et al., 2014). Although the exact mechanism mediating reduced food intake by peripherally administered GLP-1 has yet to be elucidated, it may involve signals generated by GLP-1 binding to its receptors on neurons in the gastrointestinal tract or hepatoportal bed (Burcelin et al., 2001; Holst, 2007).

4. Effect on Cardiovascular Activity. Recently, there is increasing evidence suggesting that GLP-1 may play a crucial role in the cardiovascular system (Grieve et al., 2009; Ussher and Drucker, 2014). GLP-1R is widely expressed in the heart and blood vessels, such as vascular smooth muscle, cardiomyocytes, endocardium, and coronary endothelium/smooth muscle, in both rodents and humans (Campos et al., 1994; Wei and Mojsov, 1995; Bullock et al., 1996). In an early study, treatment of adult rat cardiac myocytes with GLP-1 increased cAMP levels, but did not lead to increased cardiomyocyte contractility, as would be anticipated in the heart (Vila Petroff et al., 2001). Interestingly, treatment of mouse cardiomyocytes with GLP-1 $1_{9-36}$ amide (a GLP-1 degradation product in vivo) resulted in Akt activation, extracellular regulated kinase (ERK) phosphorylation, and reduced apoptosis induced by hypoxia or hydrogen peroxide stress, implying an unconventional action of GLP-1 (Ban et al., 2010). In a rat ischemiareperfusion model using isolated perfused heart and whole animal, GLP-1 significantly decreased infarction size, and this protection was abolished by exendin ${ }_{9-39}$, as well as inhibitors of adenylyl cyclase, phosphatidylinositol 3-kinase (PI3K), and p42/44 mitogen-activated protein kinase (MAPK), suggesting that these pathways were involved in GLP-1-mediated cardio-protection (Bose et al., 2005a). A direct action of GLP-1 on myocardial contractility and glucose uptake in normal and postischemic isolated rat hearts was also observed (Zhao et al., 2006). GLP-1 treatment significantly increased glucose uptake and decreased left ventricular end-diastolic and developed pressures. Infusion of GLP-1 into live dogs increased myocardial glucose uptake, which could be blocked by p38 MAPK kinase or endothelial nitric oxide synthase inhibitors, pointing to a direct effect of GLP-1 in myocardium (Nikolaidis et al., 2004a; Bhashyam et al., 2010). Meanwhile, GLP$1 \mathrm{R}$ was detected in human coronary artery endothelial cells and umbilical vein endothelial cells, and GLP-1 or exendin-4 treatment led to nitric oxide production in both cell types, indicative of GLP-1 involvement in the vasculature (Nystrom et al., 2004; Erdogdu et al., 2010; Ishii et al., 2014). GLP-1 infusion into rats also increased heart rate and blood pressure, thereby reflecting its direct role in the heart (Barragan et al., 1996). In recent clinical trials with either GLP-1R agonists or DPP-4 inhibitors, general beneficial effects of GLP-1 on the cardiovascular system in both normal and diabetic subjects have been revealed, an interesting finding that may lead to potential new treatment of cardiovascular diseases, although these effects require a long-term validation (van Genugten et al., 2013; Avogaro et al., 2014; Ussher and Drucker, 2014; see V. Pharmaceutical Development and Therapeutics).

5. Effect on Immune Response. GLP-1 can also regulate immune responses. Its receptor mRNA was discovered in multiple immune cell types from mice, including thymoyctes, splenocytes, bone marrow-derived cells, regulatory T cells, macrophages, and invariant natural killer T cells (Hadjiyanni et al., 2010; Hogan et al., 2011; Panjwani et al., 2013). Liraglutide treatment of patients with psoriasis, an inflammatory condition associated with metabolic diseases such as obesity, diabetes, and dyslipidemia, led to improvement of psoriasis area and severity index as well as decreased cytokine secretion from invariant natural killer $\mathrm{T}$ cells in a glycemic control-independent manner (Hogan et al., 2011). High-fat diet-fed mice treated with exendin-4 displayed decreased mRNA levels of the proinflammatory cytokines monocyte chemoattractant protein 1 , tumor necrosis factor- $\alpha$, and signal transducer and activator of transcription 3 (Koehler et al., 2009), and, in a type 1 diabetes animal model in which islets were transplanted into nonobese diabetic mice, GLP-1/gastrin 
treatment increased the number of transforming growth factor- $\beta 1$-secreting lymphocytes and decreased IFN- $\gamma-$ secreting lymphocytes with delayed onset of diabetes (Suarez-Pinzon et al., 2008).

6. Effect on Kidney Function. Some experimental data point to the participation of GLP-1 in kidney function. Infusion of GLP-1 into healthy and obese human subjects enhanced sodium excretion, urinary secretion, and glomerular filtration rate, suggesting a renal protective effect of this peptide (Gutzwiller et al., 2004). In rats, GLP-1 was able to downregulate $\mathrm{Na}^{+} / \mathrm{H}^{+}$exchanger isoform 3 (NHE3) in the renal proximal tubule, implying a potential therapeutic value for hypertension and disorders of sodium retention (Crajoinas et al., 2011). Rats administered with exendin-4 significantly improved renal function and reduced inflammation, proteinuria, and fibrosis in the kidney via a mechanism that was independent of glucose lowering (Kodera et al., 2011).

7. Effect on Nervous System. In addition to the metabolic function in the brain, GLP-1 may also exert neuroprotective and neurotropic effects (Heppner and Perez-Tilve, 2015). The aggregation of amyloid $\beta$ protein $(\mathrm{A} \beta)$ and the microtubule-associated protein Tau cause senile plaques and neurofibrillary tangles, resulting in loss of long-term potentiation, one of the major characteristics of Alzheimer's disease (AD). Infusion of GLP-1 or exendin- 4 into the lateral ventricles of mice decreased endogenous level of $\mathrm{A} \beta$, and treatment of rat hippocampus neurons with GLP-1 and exendin-4 prevented $\mathrm{A} \beta$-induced cell death (Perry et al., 2003). Intracerebroventricular dosing of GLP-1 enhanced synaptic plasticity in the hippocampus and completely reversed impairment in long-term potentiation caused by subsequent injection of $\mathrm{A} \beta$ (Gault and Holscher, 2008). GLP-1R was found in nigrostriatal neurons, and loss of these neurons is a feature of Parkinson's disease (PD). GLP-1 or exendin-4 supported cell viability during hypoxic injury in primary neurons from rat cerebral cortical tissues; this activity was blocked by GLP-19-36 (a GLP-1R antagonist) and not observed in neurons from $G L P-1^{-1-}$ mice (Li et al., 2009). Exendin-4 also maintained cell viability and reduced apoptosis caused by $\mathrm{H}_{2} \mathrm{O}_{2}$-induced oxidative stress in NSC19 neuronal cells, a spinal cord cell line with similarities to cells in the central nervous system (CNS) (Li et al., 2012b).

Obviously, GLP-1 and GLP-1R are widely expressed in many tissues, and their physiologic actions have been incrementally elucidated, especially after the extensive clinical application of GLP-1 mimetics, including GLP-1R agonists and DPP-4 inhibitors, in the last decade. Although its roles in some tissues, such as adipocytes or skeletal muscles, are still illusive, GLP1-related therapies have clearly provided multiple benefits to millions of patients suffering from metabolic disorders, and such effects are predominantly, but perhaps not always, based on signaling pathways mediated by its receptor.

\section{Structure}

Structurally, class B GPCRs consist of a large N-terminal extracellular domain (ECD) and a seven-transmembrane (7TM) helix domain, comprising the GPCR signature of seven membrane spanning $\alpha$-helices [transmembrane (TM)1-7], connected by three extracellular (ECL) and intracellular (ICL) loops, and a C-terminal helix 8 (Schioth et al., 2003; Hollenstein et al., 2014). Pharmacological studies with truncated, chimeric, and mutated ligand and receptor variants, together with peptide ligand-bound ECD crystal structures and the first 7TM crystal structures of class B GPCRs, have provided the basis for a two-domain-binding mechanism of peptide hormone ligands to secretin-like class B GPCRs (Parthier et al., 2009; Donnelly, 2012; Hollenstein et al., 2014). According to this peptide ligand-binding mechanism: 1) the $\mathrm{C}$ terminus of the peptide ligand forms an initial complex with the ECD, and this allows 2) the $\mathrm{N}$ terminus of the peptide ligand to interact with the 7TM domain (7TMD) and activate the class B GPCR to couple to G proteins and other effectors to mediate intracellular signaling processes (see III. Glucagon-Like Peptide-1 Receptor). This section gives an overview of the structureactivity relationship (SAR) of GLP-1 peptides, thereby providing important information regarding the molecular determinants of ligand binding and functional activities at the GLP-1R. The development of GLP-1 analogs is described in detail in V. Pharmaceutical Development and Therapeutics, whereas SAR of GLP$1 \mathrm{R}$ will be discussed explicitly in III. Glucagon-Like Peptide-1 Receptor. Alignments of the sequences and structures of GLP-1 with GLP-2, glucagon, GIP, and exendin-4 are presented in Fig. 2. This also annotates the structural properties of GLP-1, effects of GLP-1 mutation studies, and GLP-1R and exendin-4 interaction sites in the corresponding GLP-1R ECD crystal structures (Runge et al., 2008; Underwood et al., 2010). Peptide ligand residues shown are annotated as threeletter amino acid codes with residue number as superscripts (e.g., His ${ }^{7}$, histidine at position 7), whereas receptor residue numbers are annotated as single-letter codes, Uniprot numbers, and Ballesteros-Weinstein/Wootten numbers/secondary structure motif as superscripts, according to IUPHAR guidelines (Pawson et al., 2014) and class B GPCR residue-numbering guidelines (Wootten et al., 2013c; Isberg et al., 2015), respectively. According to the Ballesteros-Weinstein class A GPCR (Ballesteros and Weinstein, 1995) and Wootten class B GPCR (Wootten et al., 2013c) residue-numbering schemes, the single most conserved residue in each TM helix is designated X.50 (Ballesteros-Weinstein number used for comparison within class A GPCRs as well as to compare across GPCR classes) or X.50b (Wootten number for comparison with class B GPCRs). $\mathrm{X}$ is the TM helix number, and all other residues in that helix are numbered relative to this conserved position (Hollenstein et al., 2014). GLP-1R residues that 


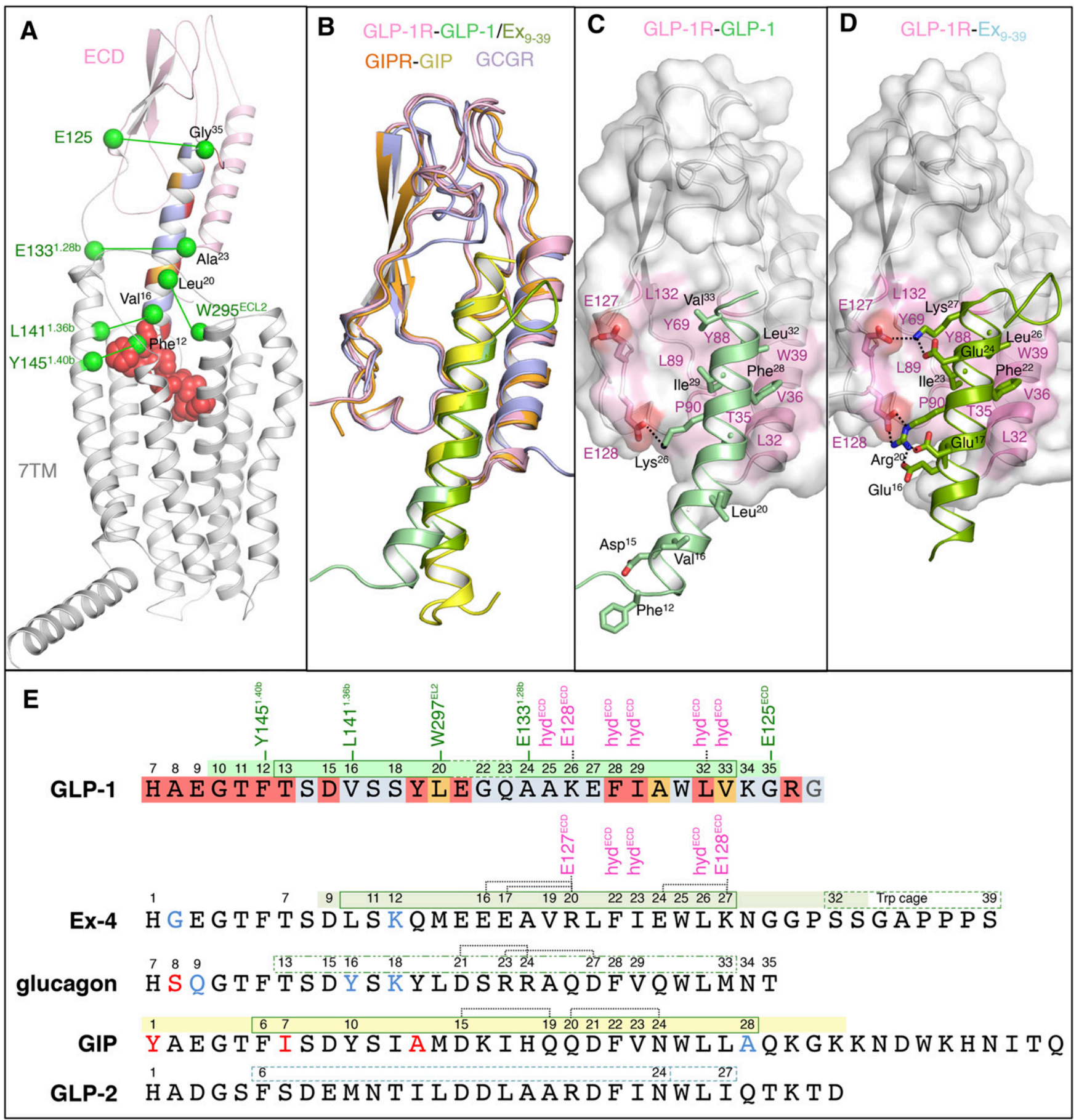

Fig. 2. Structural characteristics of GLP-1 and its cognate receptor. (A) GLP-1-bound full-length GLP-1R homology model based on a previously published full-length glucagon-bound GCGR model combining structural and experimental information from the GCGR 7TMD crystal structure (PDB: 4L6R), the GCGR ECD structure (PDB: 4ERS), and the ECD structure of GLP-1-bound GLP-1R (PDB: 3IOL), complemented by site-directed mutagenesis, electron microscopy, hydrogen-deuterium exchange, and cross-linking studies (Siu et al., 2013; Yang et al., 2015b, 2016). The C-terminal helix of GLP-1 bound to the ECD region of GLP-1R is depicted as cartoon, whereas the atoms of the flexible N-terminal region of GLP-1 predicted to be bound to the 7TMD of GLP-1R are depicted as spheres. GLP-1 is color coded according to mutation effects (blue: < fourfold effect, orange: 4- to 10 -fold effect, red: > 10-fold effect IC $_{50}$; see II. Glucagon-Like Peptide-1); mutation effects of GLP-1R are reported in Fig. 3 and Table 1. The $\mathrm{C} \alpha / \mathrm{C} \beta$ atoms of GLP-1/GLP-1R residue pairs identified in photo cross-linking studies (Chen et al., 2009, 2010b; Miller et al., 2011) are depicted

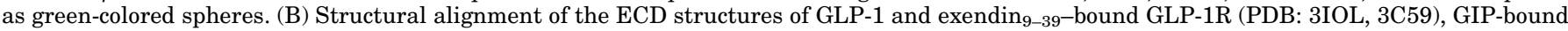
GIPR (PDB: 2QKH), and the mAb23-bound GCGR ECD structure (PDB: 4ERS). Comparison of the crystal structure binding modes of (C) GLP-1 and (D) exendin ${ }_{9-39}$. The surfaces of GLP-1R residues involved in important apolar interactions with GLP-1/apolar are colored pink, whereas residues involved in polar interactions described in II. Glucagon-Like Peptide-1 are also depicted as sticks (and their H-bond interaction networks are depicted as dashed lines). (E) Structure-based sequence alignment of GLP-1, exendin ${ }_{9-39}$, glucagon, GIP, and GLP-2. The regions of the peptide ligands solved in ECD-ligand complex crystal structures are marked above the amino acid sequences using the same color coding as in (B). Amino acids of GLP-1 are marked according to mutation study effects, as indicated in (A). The residues that are boxed are found in an $\alpha$-helical conformation in the crystal structure complex (solid lines: GLP-1, exendin ${ }_{9-39}$, GIP) or in NMR studies in micelle DPC (dashed lines: glucagon, GLP-2), as described in II. Glucagon-Like Peptide-1. 
are most conserved in secretin-like class B GPCRs are $\mathrm{S} 155^{1.50 \mathrm{~b}}, \mathrm{H} 180^{2.50 \mathrm{~b}}, \mathrm{E} 247^{3.50 \mathrm{~b}}, \mathrm{~W} 274^{4.50 \mathrm{~b}}, \mathrm{~N} 320^{5.50 \mathrm{~b}}$, G361 ${ }^{6.50 b}$, and G395 ${ }^{7.50 b}$. GLP-1 and glucagon peptide ligands start with amino acid residue $7\left(\mathrm{His}^{7}\right)$ due to posttranslational processing (Fig. 2), whereas the homologous GLP-2, GIP, and exendin-4 start with His ${ }^{1}$ and $\mathrm{Tyr}^{1}$, respectively.

1. $\alpha$-Helical C-Terminal Region. NMR, circular dichroism (CD) spectroscopy, and X-ray crystallography studies show that GLP-1 is unstructured in aqueous solution, but adopts a helical structure in a membranelike environment and by binding to its receptor (Thornton and Gorenstein, 1994; Neidigh et al., 2001; Runge et al., 2007; Underwood et al., 2010). This conformational change upon receptor binding is also proposed for other class B GPCR peptide ligands, including glucagon (Braun et al., 1983; Siu et al., 2013), GIP (Alana et al., 2006; Parthier et al., 2007), and exendin-4 (Runge et al., 2007, 2008) (Fig. 2), implying a conserved receptorligand-binding mechanism (Parthier et al., 2009). Two-dimensional NMR experiments indicate that the C-terminal region of GLP-1 $\left(\mathrm{Thr}^{13}\right.$-Lys $\left.^{34}\right)$ is in an $\alpha$-helical conformation in trifluorethanol and dodecylphosphocholine (DPC) micelles, with a less well-defined $\alpha$-helical region around $\mathrm{Gly}^{22}$ (Thornton and Gorenstein, 1994; Neidigh et al., 2001; Underwood et al., 2010). NMR studies suggest that exendin-4 adopts a more well-defined single helix ( $\mathrm{Ser}^{11}$-Lys $^{27}$ ) than GLP-1 in DPC micelles (Neidigh et al., 2001). These NMR data are in line with ligand-bound GLP-1R ECD crystal structures showing that the GLP-1 is a kinked but continuous $\alpha$-helix $\left(\mathrm{Thr}^{13}-\mathrm{Val}^{33}\right)$, whereas the truncated exendin ${ }_{9-39}$ is a straighter helix (Leu ${ }^{10}$-Lys $^{27}$ ) than GLP-1 when bound to GLP-1R (Runge et al., 2008; Underwood et al., 2010). In the ECD-bound crystal structure, the C-terminal $\alpha$-helix of GLP-1 ( $\left.\mathrm{Ala}^{24}-\mathrm{Val}^{33}\right)$ is stabilized by interaction with the ECD of GLP-1R, whereas the N-terminal helix $\left(\mathrm{Thr}^{13}-\mathrm{Glu}^{21}\right)$ does not interact with the ECD. Although it cannot be excluded that the kink in GLP-1 observed in the crystal structure is a result of crystal packing between the N-terminal part of GLP-1 (Gly ${ }^{10}$ $\mathrm{Glu}^{21}$ ) and symmetry-related ECDs, the presence of two helical segments is consistent with the SAR studies with conformationally constrained GLP-1 analogs (Miranda et al., 2008; Murage et al., 2008). Cyclization of (mutated) residues $\mathrm{Lys}^{16}-\mathrm{Glu}^{20}$ and $\mathrm{Met}^{18}-\mathrm{Ala}^{22}$ by a lactam bridge (constraining these regions in an $\alpha$-helical conformation) does not affect binding affinity and activity, whereas cyclization of residues $\mathrm{Thr}^{11}$-Asp ${ }^{15}$ even improved potency for GLP-1R compared with the corresponding linear analogs (Miranda et al., 2008; Murage et al., 2008).

Characterization of the stability and conformational changes of full-length, truncated, and GLP-1/exendin-4 chimeric peptides upon binding to the isolated ECD of GLP-1R by far-UV CD, differential scanning calorimetry, and fluorescence spectroscopy measurements demonstrated that exendin-4 has a higher $\alpha$-helical propensity than GLP-1 in solution (Runge et al., 2007). Combination of these biophysical data with pharmacological studies showed that there is a positive correlation between the stability and $\alpha$-helical propensity of the ligand in solution and its affinity for the ECD of GLP-1R (Runge et al., 2007). Comparison of the crystal structures of GLP-1-bound and exendin ${ }_{9-39}$-bound ECD provides possible explanations for the higher stability of the $\alpha$-helix of exendin- 4 as opposed to GLP-1 (Runge et al., 2008; Underwood et al., 2010). Although the $\alpha$-helix of exendin- 4 is stabilized by strong intramolecular ionic interactions between $\mathrm{Glu}^{16} / \mathrm{Glu}^{17}$ and $\mathrm{Arg}^{20}$ and between $\mathrm{Glu}^{24}$ and $\mathrm{Lys}^{27}$, corresponding $\left(\mathrm{Gln}^{23}, \mathrm{Lys}^{26}\right)$ or differentially positioned $\left(\mathrm{Glu}^{27}, \mathrm{Lys}^{34}\right)$ polar residues in GLP-1 do not form such intramolecular interactions when bound to the isolated ECD of GLP1-R [Protein Data Bank (PDB): 3IOL]. The GIPbound GIP receptor (GIPR) ECD crystal structure indicates that the $\alpha$-helix of GIP is stabilized by similar $\left(\mathrm{Asp}^{15}-\mathrm{Gln}^{19}\right)$ and alternative $\left(\mathrm{Gln}^{20}-\mathrm{Asn}^{24}\right)$ intramolecular H-bond interactions (Parthier et al., 2007), whereas glucagon may also be able to form helix-stabilizing intramolecular H-bond networks (e.g., $\mathrm{Asp}^{21}-\mathrm{Arg}^{24}$, $\mathrm{Arg}^{23}-\mathrm{Asp}^{27}$ ) (Siu et al., 2013). In addition to the higher $\alpha$-helix propensity, the more pronounced amphiphilic nature of exendin-4 enables stronger polar and hydrophobic interactions with the ECD of GLP-1R via opposite sides of the $\alpha$-helix than GLP-1 (vide infra). Moreover, exendin- 4 is eight residues longer than GLP-1, and NMR studies have shown that this extended C-terminal region, comprising $\mathrm{Ser}^{32}-\mathrm{Ser}^{39}$, forms a stable tertiary structure that folds around $\operatorname{Trp}^{25}$ in trifluorethanol and glycol (Neidigh et al., 2001). This Trp-cage conformation is, however, not observed in NMR studies in DPC micelles (Neidigh et al., 2001) and has weak electron density in the exendin $9-39$-bound ECD GLP-1R crystal structure (Runge et al., 2008), suggesting that a stable Trp-cage conformation (which is absent in GLP-1) is only partially populated in the receptor-bound state of exendin- 4 .

2. Flexible N-Terminal Region. NMR and X-ray crystallography studies indicate that the $\mathrm{N}$-terminal regions preceding the conserved $\alpha$-helix of GLP-1 (His ${ }^{7}-\mathrm{Thr}^{13}$ ), exendin-4 $\left(\mathrm{His}^{1}-\mathrm{Thr}^{7}\right)$, GIP $\left(\mathrm{Tyr}^{1}-\mathrm{Ile}^{7}\right)$, glucagon (His ${ }^{7}$ $\mathrm{Thr}^{13}$ ), and other class B GPCR peptide ligands are flexible in solution as well as in membrane-bound and ligand-bound states (Braun et al., 1983; Neidigh et al., 2001; Parthier et al., 2007; Runge et al., 2008; Underwood et al., 2010). The structure of the N-terminal region of GLP-1 $\left(\mathrm{His}^{7}-\mathrm{Thr}^{13}\right.$ ) was not clearly elucidated in NMR studies in DPC micelles due to high conformational flexibility (Neidigh et al., 2001). In the ECD-bound GLP-1 crystal structure, this $\mathrm{N}$-terminal region is unstructured, and no electron density was observed for $\mathrm{His}^{7}-\mathrm{Glu}^{9}$ (Underwood et al., 2010). Whereas the GLP-1R ECD crystal structures provide atomic details of the molecular 
interactions of the C-terminal regions of GLP-1 and exendin $_{9-39}$ with the ECD of GLP-1R (Runge et al., 2008; Underwood et al., 2010), these structures do not give information on the receptor-bound conformation of the flexible ligand $\mathrm{N}$ terminus, nor on its interactions with GLP-1R. Ligand and receptor mutation studies suggest that an extended flexible conformation of these first residues allows the peptide ligands of class B GPCRs to reach deep into the pocket (Hollenstein et al., 2014). This receptor-bound peptide conformation is proposed to be stabilized by an amino acid motif $\left(\mathrm{Thr}^{11}-\mathrm{Phe}^{12}-\mathrm{Thr}^{13}\right.$ in GLP) that is conserved in class B GPCR ligands (Neumann et al., 2008), which can induce an N-capping conformation similar to the one observed in the receptorbound NMR structure of pituitary adenylate cyclaseactivating polypeptide (PACAP) (Inooka et al., 2001). Substituting three residues in the flexible $\mathrm{N}$ terminus of GLP-1 by corresponding PACAP residues (Ala ${ }^{8} \mathrm{Ser} /$ $\mathrm{Glu}^{9} \mathrm{Asp} / \mathrm{Thr}^{11} \mathrm{Ile}$ ) does not affect affinity and potency for GLP-1R (Xiao et al., 2001), implying that GLP-1 adopts a similar conformation to PACAP upon binding to its receptor. Recently, NMR structures of an 11-mer GLP-1 analog were solved in alterative conformations containing a C-terminal $\alpha$-helix (PDB: 2N08) and an N-terminal $\beta$-turn (PDB: $2 \mathrm{~N} 09$ ), and stabilization of these conformations by cyclization cross-links (PDB: $2 \mathrm{NON}$ and 2NOI) differentially influenced GLP-1R-binding affinity and agonist potency (Hoang et al., 2015). The accumulated ligand and receptor SAR (see II. Glucagon-Like Peptide-1 and III. Glucagon-Like Peptide-1 Receptor) suggests that a flexible conformation of the first seven residues allows GLP-1 to interact with residues in the 7TM-binding pocket of GLP-1R, and that the ligand $\mathrm{N}$ terminus may adopt a more constrained conformation to activate the receptor.

3. Interaction between the C-Terminal Helix and $E C D$. Comparison of the crystal structures of GLP-1 and exendin ${ }_{9-39}$-bound GLP-1R ECD (Runge et al., 2008; Underwood et al., 2010), GIP-bound GIPR ECD (Parthier et al., 2007), and the antibody bound glucagon receptor (GCGR) ECD (Koth et al., 2012) provides information about similarities and differences in class B GPCR-bound peptide ligand conformations, and gives insights into the structural determinants of class B GPCR ligand recognition and selectivity (Fig. 2). The crystal structures of the ECDs of different class B GPCRs show that this domain has a conserved fold, including two central antiparallel $\beta$-sheets $\left(\beta_{1}-\beta_{4}\right)$ and an N-terminal $\alpha$-helix $\left(\alpha_{1}\right)$ interconnected by several loops and stabilized by three conserved disulfide bonds (Donnelly, 2012; Pal et al., 2012; Hollenstein et al., 2014) (for more detailed description of the ECD of GLP1R, see III. Glucagon-Like Peptide-1 Receptor). Class B GPCR ECD peptide ligand complexes exhibit overall a similar binding mode in which the $\mathrm{C}$ terminus of the peptide ligand adopts an $\alpha$-helical conformation that binds between $\alpha_{1}$ and $\beta_{1}-\beta_{4}$ of the ECD (Parthier et al., 2009; Donnelly, 2012; Hollenstein et al., 2014). The conserved structural fold and binding orientation suggest that common mechanisms underlie ligand recognition of class B GPCRs and indicate that peptide ligand selectivity is in part determined by specific interactions of the C-terminal $\alpha$-helix of the ligand with the ECD. The ligand-binding mode observed in the GLP-1R crystal structure is consistent with GLP-1 (vide infra) and GLP-1R (see III. Glucagon-Like Peptide-1 Receptor) mutation studies, as well as photo cross-linking experiments placing Gly ${ }^{35}$ located in the $\alpha$-helix of GLP- 1 in close proximity to $\mathrm{E} 125^{\mathrm{ECD}}$ located in the linker region between the ECD and 7TMD of GLP-1R (Chen et al., 2009). The structural details of the ECD and 7TMD of GLP-1R are discussed in detail in III. Glucagon-Like Peptide-1 Receptor.

The $\alpha$-helices of GLP-1, exendin-4, glucagon, and GIP are amphiphilic, containing a hydrophilic and hydrophobic region at opposite sides of the helix. Conserved apolar residues in the C-terminal part of GLP-1 (Phe ${ }^{28}$, $\left.\mathrm{Ile}^{29}, \mathrm{Leu}^{32}\right)$, exendin-4 $\left(\mathrm{Phe}^{22}, \mathrm{Ile}^{23}, \mathrm{Leu}^{26}\right)$, GIP $\left(\mathrm{Phe}^{22}\right.$, $\mathrm{Val}^{23}, \mathrm{Leu}^{26}$ ), and glucagon $\left(\mathrm{Phe}^{28}, \mathrm{Val}^{29}, \mathrm{Leu}^{32}\right.$ ) share a similar hydrophobic interaction site with the ECD of the corresponding receptor (GLP-1R/GIPR/GCGR: L32 ${ }^{\mathrm{ECD}} / \mathrm{A} 32 / \mathrm{M} 29$, T35 ${ }^{\mathrm{ECD}} / \mathrm{L} 35 / \mathrm{L} 32, \mathrm{~V} 6^{\mathrm{ECD}} / \mathrm{Y} 36 / \mathrm{F} 33$, and $\mathrm{W} 39^{\mathrm{ECD}} / 39 / 36$ in $\alpha_{1}, \mathrm{Y} 69^{\mathrm{ECD}} / 68 / 65$ in $\beta$-turn 1 connecting $\beta_{1}-\beta_{2}, \mathrm{Y} 88^{\mathrm{ECD}} / 87 / 84, \mathrm{~L} 89^{\mathrm{ECD}} / 88 / 85$, and $\mathrm{P} 90^{\mathrm{ECD}} / 89 /$ 86 in $\beta$-turn 2 connecting $\beta_{3}-\beta_{4}$ ) (Parthier et al., 2007; Runge et al., 2008; Underwood et al., 2010; Koth et al., 2012). Differences in hydrophobic/polar interactions with the $\mathrm{N}$-terminal part of the ligand $\alpha$-helix as well as ligand-specific ionic interactions with the ECD can partially explain the different relative affinities of GLP-1, exendin-4, GIP, and glucagon for the ECD of the corresponding receptor. The positively ionizable $\mathrm{Lys}^{26}$ of GLP-1 and homologous $\mathrm{Arg}^{20}$ of exendin ${ }_{9-39}$ both form ionic interactions with $\mathrm{E} 128^{\mathrm{ECD}}$ in the C-terminal region of the ECD of GLP-1R. The Lys ${ }^{27}$ residue of exending-39 forms an additional ionic interaction with $\mathrm{E} 128^{\mathrm{ECD}}$ in GLP-1R (Runge et al., 2008; Underwood et al., 2010), which in combination with the higher $\alpha$-helical propensity of exendin-4 compared with GLP-1 (Runge et al., 2007) might explain the increased affinity of exendin-4/exendin $9-39$ for the isolated ECD of GLP-1R compared with GLP-1. There is no ionic interaction between peptide ligand and ECD side chains observed in the GIP-bound GIPR ECD crystal structure and the ECD GCGR-glucagon docking model based on the antibody-bound GCGR and GLP-1-bound GLP-1R crystal structures. It should be noted, however, that the apolar residues of GLP-1 $\left(\mathrm{Val}^{33}\right)$, GIP $\left(\mathrm{Leu}^{27}\right)$, and glucagon $\left(\mathrm{Met}^{33}\right.$ ) that are aligned with $\mathrm{Lys}^{27}$ of exendin 9 -39 form an additional hydrophobic interaction site with the ECD of GLP-1 (Y69 $\left.{ }^{\mathrm{ECD}} / \mathrm{L} 123^{\mathrm{ECD}}\right)$, GIPR (Y68 ${ }^{\mathrm{ECD}} / \mathrm{H} 115^{\mathrm{ECD}}$ ), and GCGR (Y65 $\left.{ }^{\mathrm{ECD}} / \mathrm{A} 118^{\mathrm{ECD}}\right)$, respectively. $\mathrm{Ala}^{24}$ and $\mathrm{Ala}^{25}$ of GLP-1 and $\mathrm{Val}^{19}$ in exendin-4 form another hydrophobic interaction surface with $\alpha_{1}$ of the ECD of GLP-1R. The structurally aligned Gln ${ }^{19}$ of GIP forms 
an H-bond network with $\mathrm{Asp}^{15}$ and the N-terminal backbone of $\alpha_{1}$ of the GIPR ECD (Q30 ${ }^{\mathrm{ECD}}$ and A32 ${ }^{\mathrm{ECD}}$ ), whereas corresponding residues in glucagon $\left(\mathrm{Arg}^{24}\right.$, $\mathrm{Ala}^{25}$ ) may form a polar/apolar interaction site with the $\mathrm{N}$-terminal region of the $\alpha_{1}$ helix of GCGR (Fig. 2).

4. Interaction between Flexible $N$ terminus and $7 T M D$. The peptide ligand-bound crystal structures of the ECDs of class B GPCRs (including GLP-1- and exendin-4-bound GLP-1R, GIP-bound GIPR, and antibody-bound GCGR) do not provide information about the molecular interactions between the receptor and the flexible $\mathrm{N}$-terminal region of peptide ligands. Receptor/ligand mutagenesis and photo cross-linking studies nevertheless indicate that the 7TMD of class B GPCRs determines binding (selectivity) of the flexible $\mathrm{N}$-terminal region of peptide ligands (Donnelly, 2012; Pal et al., 2012; Hollenstein et al., 2014). A recently reported full-length GCGR-glucagon model based on the crystal structures of the GCGR 7TMD (Siu et al., 2013), the antibody-bound GCGR ECD (Koth et al., 2012), the GLP-1-bound GLP-1R ECD (Underwood et al., 2010), and the N-capped conformation of PACAP (Inooka et al., 2001) offers a template for full-length GLP-1-bound GLP-1R models (Fig. 2A) that is consistent with the results of mutation studies of GLP-1 (vide infra), GLP-1R (see III. Glucagon-Like Peptide-1 Receptor), and other class B GPCRs (Hollenstein et al., 2014). This full-length GLP-1R model furthermore satisfies spatial constraints defined by GLP-1R cross-linking studies connecting the following: 1) $\mathrm{Ala}^{24}$ in the C-terminal part of the $\alpha$-helix of GLP- 1 to $\mathrm{E} 133^{\mathrm{ECD}}$ in the region linking the ECD and 7TMD of GLP-1R (not solved in the GLP-1R crystal structure); 2) $\mathrm{Leu}^{20}$ in the N-terminal part of the $\alpha$-helix of GLP-1 to W297 ${ }^{\text {ECL2 }}$ in ECL2 between TM4 and TM5 in GLP-1R; 3) $\mathrm{Phe}^{12}$ and $\mathrm{Val}^{16}$ in the flexible N-terminal region of GLP-1 that are positioned near Y145 $5^{1.40 \mathrm{~b}}$ and L141 $1^{1.36 \mathrm{~b}}$ in the TM1 of GLP-1R, respectively; and 4) a photolabile probe at position 6 (one position before $\mathrm{His}^{7}$ ) in GLP-1 to Y205 ${ }^{\mathrm{ECL} 1}$ in ECL1 between TM2 and TM3 in GLP-1R (Chen et al., 2009, 2010b; Miller et al., 2011) (Fig. 2A).

\section{Mutagenesis}

The molecular determinants of GLP-1R ligand binding and functionality have been investigated extensively using truncated, chimeric, and site-specifically substituted GLP-1, exendin-4, and peptide ligands of closely related class B GPCRs (including glucagon and GIP). This section gives an overview as to how these data complement the structural information on GLP-1 (and the ECD-GLP-1 complex) described above. It should be noted that the current overview only focuses on changes to natural amino acids, as the incorporation of photoactive labels in GLP-1 for GLP-1/GLP-1R crosslinking studies (Chen et al., 2009, 2010b; Miller et al., 2011 ) is covered in this section, whereas the development of GLP-1 analogs by other modifications, for example, unnatural amino acid substitution and conjugation (Manandhar and Ahn, 2015), is discussed in $V$. Pharmaceutical Development and Therapeutics.

1. Truncated GLP-1 Analogs. N-terminally truncated forms of GLP-1 (including des-[His $\left.{ }^{7}\right]-$ GLP-1 ${ }_{8-36}$ and des-[His $\left.{ }^{7}, \mathrm{Ala}^{8}\right]$-GLP-19-36) are competitive antagonists of GLP-1R, whereas C-terminally truncated GLP-1 constructs (including des-[Gly $\left.{ }^{37}, \mathrm{Arg}^{36}\right]-\mathrm{GLP}-\mathrm{1}_{7-37}$ ) remain agonists (Mojsov, 1992; Montrose-Rafizadeh et al., 1997). The binding affinities of $\mathrm{N}$-terminally truncated GLP-1 constructs are not affected by mutations in the 7TMD of GLP-1R (Al-Sabah and Donnelly, 2003a,b; López de Maturana et al., 2004), indicating that interactions between the N-terminal region of GLP-1 and the 7TMD of GLP-1R are required for receptor activation. Most truncated GLP-1 constructs have a decreased affinity for GLP-1R (Mojsov, 1992; MontroseRafizadeh et al., 1997; Donnelly, 2012), suggesting that interactions with both the ECD and the 7TMD are important determinants of GLP-1 binding. Similar SARs have been observed for the endogenous ligands of related class B GPCRs, including glucagon/GCGR (Unson et al., 1989) and GIP/GIPR (Hinke et al., 2001), demonstrating that interactions of peptide ligands in the 7TMD of class B GPCRs are required for receptor activation. The N-terminally truncated des-[His $\left.{ }^{7}\right]-G L P-1_{8-36}$ and des-[His $\left.{ }^{7}, \mathrm{Ala}^{8}\right]-\mathrm{GLP}-1_{9-36}$ variants of GLP-1 have a 100- and 1000-fold lower affinity than that of wildtype, whereas truncation of more than two N-terminal residues further diminishes GLP-1R binding (Mojsov, 1992; Montrose-Rafizadeh et al., 1997). Truncation of up to three C-terminal residues $\left(\mathrm{Gly}^{35}, \mathrm{Arg}^{36}\right.$, and $\mathrm{Gly}^{37}$ ) only has a moderate effect on GLP-1R-binding affinity (up to fivefold decrease), but further deletion of C-terminal residues decreases binding affinity significantly. Nevertheless, several undecapeptide GLP- $1_{7-15}$ analogs in which the C-terminal 21 residues are replaced with a biphenylalanine dipeptide have been reported to possess almost the same potency as wild-type GLP-1 (Mapelli et al., 2009).

In contrast to GLP-1, which requires its N-terminal region for high-affinity binding to GLP-1R, the homologous agonist exendin-4 and the N-terminally truncated antagonist exendin ${ }_{9-39}$ have similar affinities for GLP-1R (Montrose-Rafizadeh et al., 1997). Although full-length GLP-1R binds exendin-4 and GLP-1 with similar high affinity, the isolated ECD maintains high

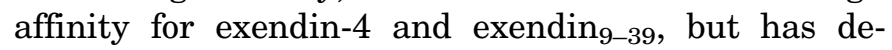
creased affinity for GLP-1 (Al-Sabah and Donnelly, 2003a; López de Maturana et al., 2003). In addition, GLP-1 binding is more sensitive to site-directed mutagenesis of the 7TMD of GLP-1R than exendin-4 (AlSabah and Donnelly, 2003a,b; López de Maturana et al., 2003; see III. Glucagon-Like Peptide-1 Receptor). Consistently, radioligand competition studies combining isolated C-terminal (ECD) and N-terminal (7TMD) GLP-1R constructs with native and N-terminally or 
C-terminally truncated GLP-1 and exendin-4 showed that: 1) GLP-1 binding is primarily determined by interactions with the ECD, but also requires interactions with the 7TMD of GLP-1R; and 2) exendin-4binding affinity is mainly determined by interactions with the ECD of GLP-1R and does not heavily depend on interactions with the 7TMD (López de Maturana et al., 2003). Based on pharmacological studies with truncated GLP-1 and exendin-4, it was further postulated that the eight-residue C-terminal extension of exendin-4 (Trp-cage, see above) may play a role in its superior affinity for the ECD of GLP-1R (Al-Sabah and Donnelly, 2003a).

2. Chimeric GLP-1 Analogs. Chimeric constructs of GLP-1 in combination with other class B GPCR peptide ligands (including exendin-4, glucagon, GIP, and PACAP) have been used to identify structural determinants of ligand selectivity for GLP-1R (and other class B GPCRs). Radioligand competition studies with truncated and chimeric GLP-1 and exendin-4 peptides suggested that divergent residues in the central part of GLP-1 $\left(\mathrm{Val}^{16}{ }^{-\mathrm{Arg}^{36}}{ }^{3}\right)$ and exendin-4 $\left(\mathrm{Leu}^{10}\right.$-Gly $\left.^{30}\right)$ determine GLP-1/exendin-4 selectivity for the ECD of GLP-1R, and indicated that the Trp-cage only plays a minor role in the increased affinity of exendin- 4 for the ECD of GLP-1R compared with GLP-1 (Runge et al., 2003b). These pharmacological data are consistent with comparative CD and fluorescence spectroscopy, NMR, and X-ray crystallography analyses of (truncated and chimeric forms of) GLP-1 and exendin-4 (Runge et al., 2007), implying that the differential affinity for the ECD can be explained by the higher $\alpha$-helical propensity of exendin- 4 in solution and by stronger (ionic) interactions between GLP-1R and exendin-4, compared with GLP-1 (see above). GLP-1/glucagon chimera experiments showed that a peptide ligand consisting of the N-terminal part of glucagon combined with the C-terminal part of GLP-1 has high affinity for both GCGR and GLP-1R (Hjorth et al., 1994). Substitution of N-terminal GLP-1 residues with corresponding glucagon residues [e.g., (Ala $\left.{ }^{8} \mathrm{Ser} / \mathrm{Glu}^{9} \mathrm{Gln}\right)$-GLP-1 and (Val ${ }^{16}$ Tyr/Ser ${ }^{12}$ Lys)-GLP-1] maintains high GLP-1Rbinding and low GCGR-binding affinities, whereas substitution of $\mathrm{N}$-terminal glucagon residues with corresponding GLP-1 residues decreases GCGR binding and maintains low GLP-1R-binding affinities. In contrast, substitution of C-terminal GLP-1 residues with corresponding glucagon residues decreases GLP$1 \mathrm{R}$ binding and maintains low GCGR-binding affinities, whereas replacement of, for example, the last three C-terminal residues of glucagon $\left(\mathrm{Met}^{33}, \mathrm{Asn}^{34}\right.$, and $\mathrm{Thr}^{35}$ ) with the corresponding GLP-1 residues $\left(\mathrm{Val}^{33}\right.$, $\mathrm{Lys}^{34}$, and $\mathrm{Gly}^{35}$ ) and an additional C-terminal GLP-1 residue ( $\mathrm{Arg}^{36}$ ) increases GLP-1R binding with affinity for GCGR remaining moderate. Glucagon/GLP-1 chimeras were combined with GCGR/ GLP-1R chimeras to identify the receptor domains that interact with $\mathrm{N}$-terminal and $\mathrm{C}$-terminal parts of glucagon and GLP-1 (Runge et al., 2003b). The chimeric GLP-1$\left(\mathrm{His}^{7}-\mathrm{Leu}^{20}\right) /$ glucagon- $\left(\mathrm{Asp}^{21}-\mathrm{Thr}^{35}\right)$ is unable to bind and activate GCGR, but its binding affinity and potency are rescued by substituting the 7TMD of GCGR with the 7TMD of GLP-1R, suggesting that the N-terminal region of GLP-1 $\left(\mathrm{His}^{7}-\mathrm{Leu}^{20}\right.$ ) interacts with the 7TMD of GLP-1R (Runge et al., 2003b). The GCGR(ECD)/ GLP-1R(7TM) chimera has equal binding affinity and potency for GLP-1 and the chimeric glucagon-(His ${ }^{7}$ $\left.\mathrm{Leu}^{20}\right) / \mathrm{GLP}-1-\left(\mathrm{Asp}^{21}-\mathrm{Gly}^{37}\right)$, although these are decreased compared with wild-type GLP-1R, indicating that the ECD of GLP-1R is the major determinant of GLP-1/glucagon selectivity by interacting with the C-terminal region of GLP-1. Chimeras combining different $\mathrm{N}$-terminal and C-terminal regions of GLP-1 $\left(\mathrm{His}^{7}-\mathrm{Leu}^{20}, \mathrm{Asp}^{21}-\mathrm{Arg}^{36}\right)$ and GIP $\left(\mathrm{His}^{1}-\mathrm{Leu}^{14}, \mathrm{Asp}^{14}\right.$ Lys $^{30}$ ), or substituting the middle parts of GIP with corresponding residues of GLP-1 ( $\mathrm{Ser}^{18}-\mathrm{Ala}^{24}, \mathrm{Glu}^{21}$. $\mathrm{Ala}^{24}$ ), all had more than 100-fold lower binding affinity for GLP1-R compared with GLP-1-(His ${ }^{7}-\mathrm{Arg}^{36}$ ) (Hareter et al., 1997). Substituting three to five residues in the N-terminal part of GLP-1 by corresponding residues from GIP (His ${ }^{7} \mathrm{Tyr} / \mathrm{Thr}^{13} \mathrm{Ile} / \mathrm{Val}^{16} \mathrm{Tyr}$ ), secretin (Ala $\left.{ }^{8} \mathrm{Ser} / \mathrm{Glu}^{9} \mathrm{Asp} / \mathrm{Asp}^{15} \mathrm{Glu} / \mathrm{Val}{ }^{16} \mathrm{Leu}\right)$, vasoactive intestinal peptide (Ala ${ }^{8} \mathrm{Ser} / \mathrm{Glu}^{9} \mathrm{Asp} / \mathrm{Gly}^{10} \mathrm{Ala} / \mathrm{Thr}{ }^{11} \mathrm{Val}$ ), or PACAP (Ala ${ }^{8}$ Ser/Glu ${ }^{9}$ Asp/Thr ${ }^{11}$ Ile/Ser ${ }^{14}$ Asp/Asp ${ }^{15}$ Ser) diminished ligand potency and decreased binding affinity by more than 10-fold (Hareter et al., 1997). Substituting two to three residues in the N-terminal part of GLP-1 by corresponding residues from glucagon ( $\mathrm{Ala}{ }^{8} \mathrm{Ser} / \mathrm{Glu}^{9} \mathrm{Gln}$ ), peptide histidine isoleucine (Glu ${ }^{9} \mathrm{Asp} /$ $\mathrm{Thr}^{11} \mathrm{Ile}$ ), or PACAP (Ala ${ }^{8} \mathrm{Ser} / \mathrm{Glu}^{9} \mathrm{Asp} / \mathrm{Thr}^{11} \mathrm{Ile}$ ), in contrast, had only small effects on affinity for GLP-1R.

3. Substituted GLP-1 Analogs. In addition to ligand (and receptor) truncation and chimera studies, several site-directed substitution experiments were performed to provide more detailed information regarding the molecular determinants of GLP-1/GLP-1R binding and selectivity.

a. GLP-1 hydrophobic region I. Point substitution of $\mathrm{Phe}^{28}$, $\mathrm{Ile}^{29}$, and $\mathrm{Leu}^{32}$ residues into Ala all had a significant negative impact on GLP-1-binding affinity and potency, confirming the important role of this hydrophobic region I in the C terminus of GLP-1 in binding the ECD of GLP-1R (Adelhorst et al., 1994; Gallwitz et al., 1994). The effect of substitution on binding affinity (GLP-1 radioligand competition $\mathrm{IC}_{50}$ ) and potency (cAMP activity $\mathrm{EC}_{50}$ ) was, however, much larger for $\mathrm{Phe}^{28}$ (1300/1000-fold decrease in affinity/ potency) and $\mathrm{Ile}^{29}$ (93/27-fold reduction) compared with $\mathrm{Leu}^{32}$ (17/twofold decrease), indicating that interactions of $\mathrm{Phe}^{28}$ and $\mathrm{Ile}^{29}$ with the conserved hydrophobic core of the ECD of GLP-1R are particularly important (Adelhorst et al., 1994). Substitution of Ala ${ }^{24}$ by Arg (the corresponding residue in glucagon) did not affect GLP-1 affinity, whereas $\mathrm{Val}^{33} \mathrm{Ala}$ (glucagon mimicking) and $\mathrm{Ala}^{30} \mathrm{Gln}$ mutations only had a moderate 5 - to 6 -fold 
effect on GLP-1 affinity (Adelhorst et al., 1994). These results imply that apolar interactions of $\mathrm{Ala}^{24}$ and $\mathrm{Val}^{33}$ with the ECD of GLP-1R [observed in the GLP-1-bound GLP-1R ECD crystal structure (Underwood et al., 2010)] are not essential for GLP-1R binding and suggest that GLP-1 residues at positions 24 (Ala/Arg) and $30(\mathrm{Ala} / \mathrm{Gln})$ are not key determinants of GLP-1R/ GCGR selectivity.

b. GLP-1 hydrophobic region II. Substitution of $\mathrm{Val}^{16}$, $\mathrm{Tyr}^{19}$, and Leu ${ }^{20}$ into Ala decreased GLP-1 affinity by sixfold $\left(\mathrm{Val}^{16}, \mathrm{Leu}^{20}\right)$ to 19 -fold $\left(\mathrm{Tyr}^{19}\right)$, and had similar negative effects on potency (Adelhorst et al., 1994), showing that $\mathrm{Tyr}^{19}$ in particular is an important interaction site in hydrophobic region II located in the middle of the GLP-1 $\alpha$-helix.

c. Polar residues in $\alpha$-helix. Alanine substitution of most polar residues in the $\alpha$-helix of GLP-1 had either no significant $\left(\operatorname{Ser}^{14}, \operatorname{Ser}^{17}, \operatorname{Ser}^{18}, \mathrm{Gly}^{22}, \mathrm{Gln}^{23}, \operatorname{Trp}^{32}\right.$ ) or only a moderate 6 -fold effect $\left(\mathrm{Lys}^{26}\right.$, $\mathrm{Lys}^{34}$ ) on GLP$1 \mathrm{R}$-binding affinity and potency, with the exception of the three negatively charged residues $\mathrm{Asp}^{15}, \mathrm{Glu}^{21}$, and $\mathrm{Glu}^{27}$ (Adelhorst et al., 1994). Replacement of Asp ${ }^{15}$ by Ala resulted in a 41-fold decrease in affinity and loss of potency (Adelhorst et al., 1994). A recent systematic mutation study combining GLP-1 (Asp ${ }^{15} \mathrm{Glu}, \mathrm{Asp}^{15} \mathrm{Lys}$, $\mathrm{Asp}^{15} \mathrm{Arg}$ ) and GLP-1R (residues L379 ${ }^{\mathrm{ECL} 3} \mathrm{R} / \mathrm{E}$, R380 ${ }^{\mathrm{ECL} 3} \mathrm{D} / \mathrm{G}, \mathrm{F} 381^{\mathrm{ECL} 3} \mathrm{R} / \mathrm{E}$ ) demonstrated that an ionic interaction between $\mathrm{Asp}^{15}$ and $\mathrm{R}^{15} \mathrm{O}^{\mathrm{ECL} 3}$ in the third extracellular loop of GLP-1R is indeed required for ligand recognition and receptor activation (Moon et al., 2015). The diminished GLP-1 binding of the GLP-1R $\mathrm{R} 380^{\text {ECL3 }} \mathrm{D}$ mutant was partially restored by $\mathrm{Asp}^{15} \mathrm{Glu}$ substitution and almost fully restored by the Asp ${ }^{15} \mathrm{Lys}$ and inverted $\mathrm{Asp}^{15} \mathrm{Arg}$ substitutions of GLP-1. The abolished potency of GLP-1 for the GLP-1R R380 ${ }^{\mathrm{ECL} 3} \mathrm{D}$ was partially restored by the Asp ${ }^{15}$ Lys and inverted Asp ${ }^{15}$ Arg substitutions. Replacement of $\mathrm{Glu}^{21}$ by Ala and Gly significantly decreased GLP-1 affinity by 15-fold and 60-fold, respectively (Adelhorst et al., 1994; Watanabe et al., 1994), indicating that the side chain of $\mathrm{Glu}^{21}$ plays a key role in GLP-1 binding. $\mathrm{Glu}^{27} \mathrm{Ala}$ substitution had a moderate sixfold effect on binding affinity, but a greater, 240-fold, effect on potency, albeit with a large S.E.M. in the $\mathrm{EC}_{50}$ of the mutant (Adelhorst et al., 1994). Replacement of $\mathrm{Glu}^{27}$ by Lys did not have a significant effect on binding affinity or potency (Watanabe et al., 1994), suggesting that a charged/polar residue at position 27 may facilitate GLP$1 \mathrm{R}$ activation, possibly by stabilizing the $\alpha$-helix of GLP-1 and/or stabilizing the active conformation of GLP-1R.

d. $N$-capping motif in GLP-1. Alanine substitution of $\mathrm{Thr}^{11}$, Phe ${ }^{12}$, and $\mathrm{Thr}^{13}$, the three residues in GLP-1 that are proposed to stabilize the N-capped conformation (Inooka et al., 2001; Neumann et al., 2008) of the N terminus of GLP-1, had a significant impact on ligand affinity. The effect of the Thr ${ }^{11}$ Ala substitution was, however, smaller (13-fold decrease) compared with $\mathrm{Phe}^{12} \mathrm{Ala}$ and $\mathrm{Thr}^{13}$ Ala substitutions (133-fold decrease), whereas GLP-1 potency was only significantly affected by the latter two substitutions. These results suggest that $\mathrm{Phe}^{12}$ and $\mathrm{Thr}^{13}$ play a more important role in stabilizing the $\mathrm{N}$-capped conformation via intramolecular interactions within GLP-1 and/or in stabilizing the activated conformation of GLP-1R via intermolecular interactions with the receptor.

e. $N$ terminus of GLP-1. Substitution studies indicate that the electrostatic, steric, and conformational properties of the four residues following the $\mathrm{N}$-capping motif of GLP-1 $\left(\mathrm{His}^{7}, \mathrm{Ala}^{8}, \mathrm{Glu}^{9}, \mathrm{Gly}^{10}\right)$ are important determinants for GLP-1R binding and/or activation. Substitution of $\mathrm{His}^{7}$ by Phe did not affect binding affinity or ligand potency, whereas substitution with positively ionizable (Arg, Lys), smaller (Ala), or larger (Trp, GIP-mimicking Tyr) residues diminished binding affinity (Adelhorst et al., 1994; Hareter et al., 1997; Gallwitz et al., 2000; Sarrauste de Menthiere et al., 2004). Altogether, these amino acid replacement data suggest that a small aromatic residue is required at position 7 that is sterically compatible with the GLP1-R binding site. The $\mathrm{Ala}^{8}-\mathrm{Val}$ and the exendin-4-mimicking $\mathrm{Ala}^{8}$-Gly mutants only showed a small (two- to threefold) decrease in binding affinity and potency, the $\mathrm{Ala}^{8}$-Ser analog displayed moderate to large impact (4- to 10-fold) on binding affinity, whereas substitution of $\mathrm{Ala}^{8}$ with Leu or Thr reduced GLP-1R binding (Adelhorst et al., 1994; Hareter et al., 1997; Deacon et al., 1998; Burcelin et al., 1999). These observations demonstrate that only small residues are tolerated at position 8 , suggesting that steric constraints of the GLP-1R binding site and/or conformational flexibility of the $\mathrm{N}$-terminal region around $\mathrm{Ala}^{8}$ play a crucial role in GLP-1 binding. Substitution of $\mathrm{Glu}^{9}$ with Asp, Met, or Leu did not significantly affect ligand affinity/potency; substitution with Ser, Tyr, Phe, or Pro moderately decreased affinity (5- to 10-fold); whereas replacement with Ala, Lys, and Val reduced ligand binding (Xiao et al., 2001; Green et al., 2003, 2004; Sarrauste de Menthiere et al., 2004). The substitution data of $\mathrm{Glu}^{9}$ indicate that residues with similar negative charge (Asp) or comparable size (Met, Leu) are preferred, and positively charged moieties (Lys) are not tolerated at position 9. Substitution of $\mathrm{Gly}^{10}$ by Ala, Asp, Glu, His, Lys, or Arg diminished both GLP-1 affinity and potency (Adelhorst et al., 1994; Moon et al., 2015), demonstrating the essential function of this residue in GLP-1R binding. Combined GLP-1 and GLP-1R mutation studies showed that diminished binding affinity and potency of GLP-1 for the GLP-1R R380 ${ }^{\mathrm{ECL} 3} \mathrm{D}$ mutant could, for a small part, be recovered by the GLP-1 $\mathrm{Gly}^{10}$-Arg mutant (Moon et al., 2015), indicating that the Gly/Arg ${ }^{10}$ is in the vicinity of R/D380 ${ }^{\text {ECL3 }}$.

In summary, combining GLP-1 truncation, chimera, and point substitution studies presented in this section with structural information on GLP-1 conformation and interactions with GLP-1R and GLP-1R mutation data, a 
binding model for the full-length GLP-1-GLP-1R complex can be proposed (Fig. 2), in which: 1) hydrophobic region I in the C-terminal $\alpha$-helix of GLP-1 consisting of $\mathrm{Phe}^{28}, \mathrm{Ile}^{29}$, and Leu ${ }^{32}$ binds the ECD (as observed in the GLP-1-bound GLP-1R ECD crystal structure); 2) a second hydrophobic region II in the middle of GLP-1 $\left(\mathrm{Val}^{16}, \mathrm{Tyr}^{19}, \mathrm{Leu}^{20}\right)$ interacts with the region connecting the ECD and 7TMD (the so-called stalk region in the GCGR crystal structure); 3) the $\mathrm{N}$ terminus (His ${ }^{7}-\mathrm{Thr}^{13}$ ) of glucagon interacts with the 7TMD in a so-called N-capped conformation (stabilized by $\mathrm{Thr}^{11}, \mathrm{Phe}^{12}, \mathrm{Thr}^{13}$ ). In addition to these main determinants of GLP-1/GLP-1R binding, other residues in the $\mathrm{C}$-terminal $/ \alpha$-helical region can play an important role in stabilizing and maintaining the amphiphilic character of the $\alpha$-helix of GLP- 1 or by forming additional hydrophobic ( $\mathrm{Ala}^{24} / \mathrm{Ala}^{25}$ with the ECD) or ionic/polar ( Lys $^{26}$ with the ECD, $D^{15}$ with ECL3) interaction sites with GLP-1R.

\section{Glucagon-Like Peptide 1 Receptor}

\section{A. Discovery}

Between 1979 and early 1980, Habener and colleagues found that 29-amino-acid pancreatic glucagon was the major bioactive peptide released from the pancreatic islets (Goodman et al., 1980a; Lund, 2005). The anglerfish islet was thus regarded as a rich source containing coding sequences for glucagon-related peptides. Hybrid arrest and hybrid selection of mRNAs encoding two preproglucagons led to the first identification of a cDNA encoding a 29-residue peptide highly homologous to mammalian glucagon (Goodman et al., 1980b; Lund et al., 1981), which was then used as a hybridization probe to screen the anglerfish islet cDNA library for other cDNAs to derive the entire coding sequence of anglerfish preproglucagon (Lund et al., 1982).

In 1982, the first mammalian (hamster) pancreatic preproglucagon cDNA was reported (Bell et al., 1983a), which resulted in the rapid isolation and sequencing of the human glucagon gene (Bell et al., 1983b), followed by bovine and rat preproglucagon cDNAs (Heinrich et al., 1984). These landmark discoveries led to our current concepts of GLP-1 as an incretin and a satiety hormone (Ebert and Creutzfeldt, 1987; Kreymann et al., 1987; Dupre et al., 1991; Mattson et al., 2003). It was found that truncated forms of GLP-1 are also biologically active in stimulating both insulin gene transcription and insulin secretion (Mojsov et al., 1986; Drucker et al., 1987; Fehmann and Habener, 1991; II. GlucagonLike Peptide-1).

1. Receptor Cloning. To better understand the action of GLP-1, Thorens isolated and characterized the first cDNA of the rat pancreatic $\beta$ cell GLP-1 receptor in 1992 (Thorens, 1992), followed by cloning of the human receptor in 1993 (Dillon et al., 1993; Graziano et al., 1993; Thorens et al., 1993), revealing a sequence of 463 residues. This deduced primary sequence resembled that of the receptors for secretin, parathyroid hormone, and calcitonin, and hence enabled it to be classified within what was then a new branch of the GPCR superfamily (family B, class B, or secretin receptor-like) that now includes 15 members (Segre and Goldring, 1993; Hoare, 2005).

2. Receptor Expression. Using RNA enzyme protection techniques, GLP-1R was found widely expressed in the human and mouse pancreas, lung, brain, stomach, heart, and kidney, but was not seen in tissues involved in glucose metabolism, such as the liver, skeletal muscle, and fat (Dunphy et al., 1998). DNA hybridization experiments showed that the human GLP-1R ( $h G L P-1 R)$ gene is localized to chromosome $6 \mathrm{p} 21$, and the size is $40 \mathrm{~kb}$, containing about 14 exons. Both human and rat receptors contain 463 amino acids, whereas that of the mouse has 489 amino acids, displaying a homology with hGLP$1 \mathrm{R}$ of $91 \%$ and $84 \%$, respectively. Although the expression levels of GLP-1R vary among different tissues and cell types, Northern hybridization studies demonstrated that its expression in pancreatic islets, heart, and lungs is significantly higher (Dunphy et al., 1998). In addition, GLP-1R was also found in the lateral septum, thalamus, and hippocampus of the rat brain, and the cDNA fragment cloned from the brain, heart, and pancreas encodes the same amino acids of the receptor, suggesting its physiologic relevance in the cardiovascular and central nervous systems (Satoh et al., 2000).

It is known that the expression of GLP-1R in the islets of Langerhans is regulated by glucose and dexamethasone, but not by the PKA-dependent signaling pathway (Abrahamsen et al., 1995). Starvation and refeeding could influence its expression in the rat hypothalamus and other sites of the CNS (MacLusky et al., 2000). Although our knowledge about the transcriptional regulation mechanisms of GLP-1R is still limited, promoter analysis indicate that 1 ) transcription factors $\mathrm{Sp} 1$ and Sp3 may play important regulatory roles and 2) homologous desensitization and internalization are closely related to the intracellular sites of 441/442, 444/445, and 451/452 (Wildhage et al., 1999).

3. Receptor Biosynthesis. About 5-10\% of GPCRs contain an N-terminal cleavable signal peptide (Schulein et al., 2012). Most GPCRs mediate the integration of receptor into cell membrane by a signal anchor sequence that is located at the first TMD (Audigier et al., 1987; Wallin and von Heijne, 1995). Both types of signal sequences regulate the endoplasmic reticulum (ER) signal process at the beginning of the secretory pathway (Belin et al., 1996; Kochl et al., 2002).

The signal peptide is a sequence of approximately 20 amino acids in the $\mathrm{N}$ terminus of the receptor that includes a polar and charged N-terminal (n) region, a central hydrophobic (h) region, and a polar C-terminal (c) region (Schneider and Fechner, 2004; Clerico et al., 2008). The C-terminal side regularly contains helixbreaking proline and glycine residues and small uncharged residues at positions 1 and 3 of the cleavage 
site. After synthesis of the receptor's N-tail in the cytoplasm, the translation ceases by its binding to the signal recognition particle (SRP) (Halic and Beckmann, 2005; Shan and Walter, 2005). A translocon complex is attached to the membranes of ER that includes a GTPdependent interaction between SRP and SRP receptor, translocon gating, protein synthesis, and integration of nascent chains with the bilayer (Kochl et al., 2002). The signal peptide is finally cleaved off by signal peptidases on the ER membrane. In contrast, signal anchor sequences are not cleaved and form a part of the mature protein (Audigier et al., 1987; Wallin and von Heijne, 1995; Higy et al., 2004; Schulein et al., 2012). In addition to recognition and binding of SRP and translocation to $\mathrm{ER}$, the N-terminal signal sequence may play key roles in protein folding and trafficking. This is highly dependent upon whether the signal sequence is a cleaved signal peptide or an uncleaved signal anchor.

For GLP-1R, studies on the signal peptides of corticotropin-releasing factor-1 receptor $\left(\mathrm{CRF}_{1} \mathrm{R}\right)$ and corticotropin-releasing factor-2 receptor give a good model to compare. Both CRF receptors exhibit a high probability of N-terminal cleavable signal peptide by computational prediction. In reality, however, only the signal peptide of $\mathrm{CRF}_{1} \mathrm{R}$ is cleaved, whereas that of corticotropin-releasing factor-2 receptor is translocated and embedded to the plasma membrane by taking TM1 as a signal anchor sequence. It is thus integrated to be a part of the N-terminal ECD: not mediating ER targeting, but contributing to receptor activation (Alken et al., 2005). Interestingly, a mutant of rat $\mathrm{CRF}_{1} \mathrm{R}$ without the signal peptide sequence was able to translocate to the cell surface and display similar biologic function to the wild-type. The rat $\mathrm{CRF}_{2 \mathrm{a}} \mathrm{R}$ contains an uncleaved $\mathrm{N}$-terminal signal peptide, and deletion of this resulted in a significant reduction in its membrane expression, suggesting that receptor transportation was affected (Rutz et al., 2006). The complexity of these signal sequences highlights the need for experimental verification of the role of the signal sequence in GLP-1R.

According to the signal peptide prediction program "SignalP 4.0" (Nielsen et al., 1997; Bendtsen et al., 2004), the sequence of the first 23 amino acids in GLP$1 \mathrm{R}$ fits all the criteria of an $\mathrm{N}$-terminal signal peptide. The existence of a functional signal peptide was demonstrated experimentally by Huang et al. (2010), who showed that it was required for GLP-1R synthesis and was cleaved thereafter. Mutation of the signal peptide (A21-R) resulted in retention of the receptor within ER, whereas mutation of E34-R augmented its cell surface expression when the signal peptide was deleted. The amino acid sequence following the signal peptide in the GLP-1R, G27 ${ }^{\mathrm{ECD}}-\mathrm{W} 39^{\mathrm{ECD}}$, is relatively hydrophobic, and this region may be recognized by SRP (Huang et al., 2010). Ge et al. (2014) went deep into the function of this signal peptide by use of constructs containing epitope tags at the $\mathrm{N}$ and/or $\mathrm{C}$ terminus. A mutant GLP-1R without the signal peptide sequence was expressed in human embryonic kidney (HEK) 293 cells and displayed normal functionality with respect to ligand binding and cAMP activation, suggesting that the putative signal peptide may not be required for receptor synthesis. Immunoblotting analysis showed that the amount of GLP-1R synthesized in HEK293 cells was low without the signal peptide, indicating its role in facilitating receptor expression (Ge et al., 2014). Epitope tags at the N terminus of GLP-1R were detectable by immunofluorescence and immunoblotting, an observation that is inconsistent with another report that studied both signal peptide and the hydrophobic region after the signal peptide. It was found that the signal peptide was cleaved in the mature hGLP-1R, and cell surface expression was almost abolished by the mutation A21- $R$ that prevented the cleavage, demonstrating that hydrophobic region after the signal peptide is necessary for efficient hGLP-1R trafficking to the cell surface. Because glycosylation is vital to cell surface expression, cleavage of the signal peptide will affect this process. In addition, mutating $\mathrm{W} 39^{\mathrm{ECD}}, \mathrm{Y} 69^{\mathrm{ECD}}$, and Y $88^{\mathrm{ECD}}$ of hGLP-1R to alanine eliminated its cell surface expression without influencing N-linked glycosylation and cleavage of the signal peptide (Thompson and Kanamarlapudi, 2014).

Clearly, such discrepancies may not only reflect differences in the methods employed among these three studies, but also the cellular background and the complexity of the underlying mechanism(s).

\section{B. Structure}

The human GLP-1R is a 463-residue glycoprotein containing an $\mathrm{N}$-terminal signal peptide and various glycosylation sites that are essential for the correct trafficking and processing of the receptor (Thorens, 1992; Dillon et al., 1993; Graziano et al., 1993; Thorens et al., 1993; Goke et al., 1994; Chen et al., 2010a; Huang et al., 2010). Like all class B GPCRs, GLP-1R possesses an N-terminal ECD of 100-150 residues connected to an integral TM domain (7TM) that is typical of all GPCRs, having seven $\alpha$-helices (TM1-TM7) separated by ICL1-ICL3 and ECL1-ECL3 (Palczewski et al., 2000; Siu et al., 2013). However, despite this structural resemblance to other GPCRs, the sequence of the class B 7TMD is devoid of the consensus sequence motifs that typify the class A GPCRs (e.g., rhodopsin and adrenergic receptors). In contrast, the $\mathrm{N}$-terminal ECD of class B GPCRs is a unique domain found only in this GPCR subfamily and is central to ligand recognition. Class B GPCRs are believed to all bind their peptide ligands via a common mechanism known as the two-domain model, in which the ECD first binds to the $\mathrm{C}$-terminal region of the ligand, enabling a second interaction between the $\mathrm{N}$-terminal region of the ligand and the 7TMD of the receptor (Bergwitz et al., 1996; Hoare, 2005). 


\section{N-Terminal Domain.}

a. Structure determination of the N-terminal ECD. The two-domain structure of GLP-1R enabled a strategy whereby the ECD could be expressed as an isolated domain suitable for structural and functional studies that were used to demonstrate its critical role in ligand binding (Wilmen et al., 1996; Xiao et al., 2000; Bazarsuren et al., 2002; Al-Sabah and Donnelly, 2003a; López de Maturana et al., 2003; Mann et al., 2007, 2010b; Runge et al., 2007, 2008; Underwood et al., 2010). The definitive study came from X-ray crystallography whereby the isolated ECD of hGLP-1R from an Escherichia coli inclusion body preparation was incubated with the peptide antagonist exendin ${ }_{9-39}$, before being further purified, crystallized, and analyzed using X-ray diffraction to yield a $2.2 \AA$ crystal structure (Runge et al., 2008). The protein fold closely resembles that of other class B GPCR ECD structures (Parthier et al., 2009) and contained two regions of antiparallel $\beta$-sheet, three disulfide bonds $\left(46^{\mathrm{ECD}}-71^{\mathrm{ECD}}, 62^{\mathrm{ECD}}-104^{\mathrm{ECD}}\right.$, and $\left.85^{\mathrm{ECD}}-126^{\mathrm{ECD}}\right)$, and an N-terminal $\alpha$-helix. The core of the structure contains six conserved residues $\left(\mathrm{D} 67^{\mathrm{ECD}}\right.$, W72 ${ }^{\mathrm{ECD}}$, $\mathrm{P} 86^{\mathrm{ECD}}$, R102 ${ }^{\mathrm{ECD}}, \mathrm{G} 108^{\mathrm{ECD}}$, and $\mathrm{W} 110^{\mathrm{ECD}}$ ), which are critical for the folding stability. Residues on the $\alpha$-helix, turn 1 , loop 2 , and the $\mathrm{C}$-terminal region form a ligand-binding groove for the antagonist's well-defined $\alpha$-helix $\left(\mathrm{Leu}^{10}-\mathrm{Asn}^{28}\right)$ that interacts with the ECD using residues within the Glu $15^{\mathrm{ECD}}-\mathrm{Ser} 32^{\mathrm{ECD}}$ region, the most critical residues being Val19 ${ }^{\mathrm{ECD}}$, Phe $22^{\mathrm{ECD}}$, Ile $23^{\mathrm{ECD}}$, and Leu $26^{\mathrm{ECD}}$, which are deeply buried in the ECD's groove. The residues on the N-terminal side of $\mathrm{Glu}^{15}$ of exendin ${ }_{9-39}$ do not interact with the ECD, although residues 10-14 are nevertheless critical for high-affinity binding (Runge et al., 2007), presumably because they are required to stabilize the helical structure of the ligand. The nine-residue $\mathrm{C}$-terminal extension of exendin ${ }_{9-39}$, which has no equivalent in GLP-1, plays no significant role in the peptide's affinity at hGLP-1R (Runge et al., 2007; Mann et al., 2010b). A later crystal structure showed that GLP-1 also forms an $\alpha$-helix when bound to the ECD, with the principal contacts being the equivalent hydrophobic interface formed by residues $\mathrm{Ala}^{24}, \mathrm{Ala}^{25}, \mathrm{Phe}^{28}, \mathrm{Ile}^{29}, \mathrm{Leu}^{32}$, and $\mathrm{Val}^{33}$ (Underwood et al., 2010). In contrast to that in exendin ${ }_{9-39}$, the ECD-bound $\alpha$-helix of GLP-1 is kinked around $\mathrm{Gly}^{22}$, as observed in earlier NMR studies (Thornton and Gorenstein, 1994). The modest eightfold differential affinity between exendin- 4 and GLP-1 at the isolated membrane-bound ECD of hGLP1R (Mann et al., 2010b) could largely be explained through $\mathrm{E} 127^{\mathrm{ECD}}$, which interacts with exendin ${ }_{9-39}$ but not GLP-1, where mutagenesis to Ala resulted in a sevenfold reduction in affinity for exendin ${ }_{9-39}$ (Underwood et al., 2010; Patterson et al., 2013). Leu32 ${ }^{\mathrm{ECD}}$ also appears to play a role in ligand selectivity, although it is less clear from the crystal structure how this occurs: substitution of Leu $32^{\mathrm{ECD}}$ by Ala had no effect upon GLP-1 affinity or potency but did reduce exendin ${ }_{9-39}$ affinity by sevenfold and $\mathrm{Gly}^{2}-$ GLP-1 by 10-fold (Underwood et al., 2010; Patterson et al., 2013).

b. Site-directed mutagenesis of the ECD. The $\mathrm{N}$-terminal domain has been the subject of a number of mutagenesis studies summarized in Table 1 and Fig. 3 (Wilmen et al., 1997; Tibaduiza et al., 2001; Mann et al., 2010b; Underwood et al., 2010; Day et al., 2011; Koole et al., 2011; Patterson et al., 2013). Residues that have been mutated but do not greatly affect GLP-1 affinity/efficacy include $\mathrm{P}^{\mathrm{ECD}} \mathrm{L}, \mathrm{R} 20^{\mathrm{ECD}} \mathrm{K}, \mathrm{L} 32^{\mathrm{ECD}} \mathrm{A}$, T35 ${ }^{\mathrm{ECD}} \mathrm{A}, \mathrm{V} 36^{\mathrm{ECD}} \mathrm{A}, \mathrm{R} 44^{\mathrm{ECD}} \mathrm{H}, \mathrm{E} 68^{\mathrm{ECD}} \mathrm{A}, \mathrm{L} 123^{\mathrm{ECD}} \mathrm{A}$, $\mathrm{E} 127^{\mathrm{ECD}} \mathrm{A}, \mathrm{E} 127^{\mathrm{ECD}} \mathrm{Q}, \mathrm{E} 128^{\mathrm{ECD}} \mathrm{A}, \mathrm{E} 128^{\mathrm{ECD}} \mathrm{Q}$, and E128 ${ }^{\mathrm{ECD}} \mathrm{M}$. To study a species-selective small-molecule antagonist, Tibaduiza et al. (2001) mutated W33 ${ }^{\mathrm{ECD}}$ to Ser (the human to rat GLP-1R substitution), as well as to various other residue types, showing that there was no effect on GLP-1 albeit that the affinity and potency values were not given for the peptide ligands used. Wilmen et al. (1997) substituted six Trp residues in the ECD with Ala and found that, whereas W87A behaved like wild-type GLP-1R in binding and cAMP assays, the remaining substitutions at $39,72,91,110$, and 120 resulted in the abolition of detectable radioligand binding. Modest five- to eightfold reductions in GLP-1 potency have been observed for $\mathrm{E} 68^{\mathrm{ECD}} \mathrm{K}, \mathrm{P} 90^{\mathrm{ECD}} \mathrm{A}$, and R121 ${ }^{\mathrm{ECD}} \mathrm{A}$ (Underwood et al., 2010; Day et al., 2011). The ECD structures suggest that $\mathrm{E} 68^{\mathrm{ECD}}$ is close to the C-terminal region of the peptide ligand, but, although it appears to have little significant interaction with exendin $_{9-39}$ in the human receptor, the equivalent residue in rat GLP-1R, D68 ${ }^{\mathrm{ECD}}$, enhances exendin ${ }_{9-39}$ affinity by forming a hydrogen bond with $\mathrm{Ser}^{32}$ of the peptide (Mann et al., 2010b). Other mutations, Y $69^{\mathrm{ECD}} \mathrm{A}$, $\mathrm{Y} 88^{\mathrm{ECD}} \mathrm{A}$, and $\mathrm{L} 89^{\mathrm{ECD}} \mathrm{A}$, have caused catastrophic effects on the ability of the receptor to be detected in binding or signaling assays (Underwood et al., 2010).

\section{Seven-Transmembrane Domain.}

a. Structure of the 7TMD. To date, with the exception of a recently solved electron microscopy map of GCGR (Yang et al., 2015b), there are no experimentally determined three-dimensional structures available for any full-length class B GCPR, but, in addition to the isolated ECD structures mentioned above, there are now X-ray crystal structures for the 7TMD of human GCGR (Siu et al., 2013; Jazayeri et al., 2016) and $\mathrm{CRF}_{1} \mathrm{R}$ (Hollenstein et al., 2013). The partial $\mathrm{CRF}_{1} \mathrm{R}$ structure (PDB: 4K5Y) was solved in the presence of a smallmolecule antagonist (CP-376395) and contained 12 thermo-stabilizing mutations and a T4 lysozyme fusion partner inserted into ICL2 (Hollenstein et al., 2013). The first GCGR 7TMD structure (PDB: 4L6R) was solved with an N-terminal fusion partner fused at residue 123 (Siu et al., 2013), and it is this structure that is of particular interest to understand GLP-1R because both the receptors and native ligands are closely related. Recently, another GCGR 7TMD crystal structure was 
TABLE 1

Summary of effects on GLP-1 pharmacology (affinity and ability to activate cAMP pathway) in published site-directed mutagenesis studies of GLP-1R

WT (wild-type) refers to mutations that resulted in either < fivefold or no statistically significant change from wild-type GLP-1R. ND (not determinable) refers to a property

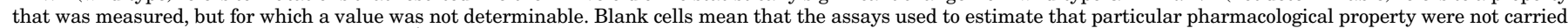

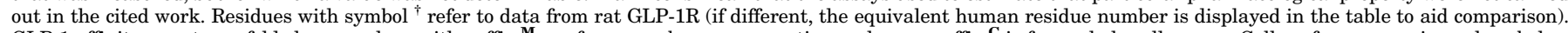

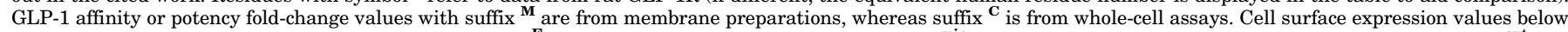

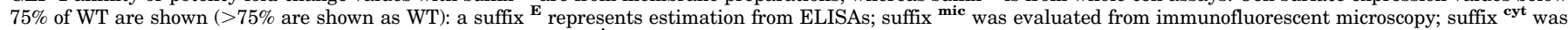

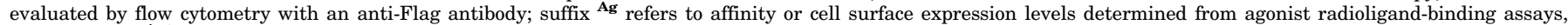

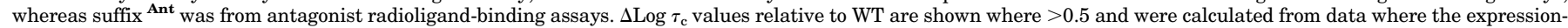

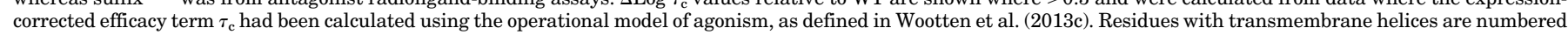
according to Wootten et al. (2013c).

\begin{tabular}{|c|c|c|c|c|c|c|}
\hline Residue & $\begin{array}{l}\text { Mutated } \\
\text { to }\end{array}$ & $\begin{array}{l}\text {-Fold Reduction } \\
\text { Affinity }\end{array}$ & $\begin{array}{l}\text {-Fold Reduction } \\
\text { Potency }^{*}\end{array}$ & $\begin{array}{l}\text { Cell Surface } \\
\text { Expression } \\
(\% \text { Wild-Type) }\end{array}$ & $\begin{array}{l}\text { Comments and/or Other } \\
\text { Observed Effects }\end{array}$ & Reference \\
\hline $\mathrm{P} 7^{\mathrm{ECD}}$ & $\mathrm{L}$ & $\mathrm{WT}^{\mathrm{C}, \mathrm{Ant}}$ & WT & $\mathrm{WT}^{\mathrm{E}}$ & & Koole et al., 2011 \\
\hline $\mathrm{R} 20^{\mathrm{ECD}}$ & $\mathrm{K}$ & $\mathrm{WT}^{\mathrm{C}, \mathrm{Ant}}$ & WT & $\mathrm{WT}^{\mathrm{E}}$ & & Koole et al., 2011 \\
\hline $\mathrm{L} 32^{\mathrm{ECD}}$ & $\mathrm{A}$ & $\mathrm{WT}^{\mathrm{M}, \mathrm{Ag}}$ & WT & $\mathrm{WT}^{\mathrm{Ag}}$ & & Underwood et al., 2010 \\
\hline $\mathrm{L} 32^{\mathrm{ECD}}$ & $\mathrm{A}$ & & WT & & & Patterson et al., 2013 \\
\hline $\mathrm{W} 33^{\mathrm{ECD}}$ & $\mathrm{S}$ & $\mathrm{WT}^{\mathrm{C}, \mathrm{Ant}}$ & & & Species change (human to & Tibaduiza et al., 2001 \\
\hline
\end{tabular}

\begin{tabular}{|c|c|c|c|c|c|c|}
\hline & & & & & data are shown & \\
\hline $\mathrm{T} 35^{\mathrm{ECD}}$ & A & WT & & $7 \% \mathrm{Ag}$ & & Underwood et al., 2010 \\
\hline Val-36 $6^{\mathrm{ECD}}$ & $\mathrm{A}$ & $\mathrm{WT}^{\mathrm{M}, \mathrm{Ag}}$ & WT & $\mathrm{WT}^{\mathrm{Ag}}$ & & Underwood et al., 2010 \\
\hline $\mathrm{W} 39^{\mathrm{ECD} \dagger}$ & & $\mathrm{ND}^{\mathrm{C}, \mathrm{Ag}}$ & & & $\begin{array}{c}\text { Membrane expression } \\
\text { confirmed via WB }\end{array}$ & Wilmen et al., 1997 \\
\hline $\mathrm{R} 44^{\mathrm{ECD}}$ & $\mathrm{H}$ & $\mathrm{WT}^{\mathrm{C}, \mathrm{Ant}}$ & WT & $\mathrm{WT}^{\mathrm{E}}$ & & Koole et al., 2011 \\
\hline$N 63^{\mathrm{ECD}}$ & $\mathrm{L}$ & $\mathrm{WT}^{\mathrm{C}, \mathrm{Ag}}$ & WT & $\mathrm{WT}^{\mathrm{Ag}}$ & & Chen et al., 2010a \\
\hline $\mathrm{E} 68^{\mathrm{ECD}}$ & $\mathrm{A}$ & $\mathrm{WT}^{\mathrm{M}, \mathrm{Ag}}$ & WT & $\mathrm{WT}^{\mathrm{Ag}}$ & & Underwood et al., 2010 \\
\hline $\mathrm{E} 68^{\mathrm{ECD}}$ & $\mathrm{K}$ & & 8 & & & Day et al., 2011 \\
\hline Y $69^{\mathrm{ECD}}$ & $\mathrm{A}$ & $\mathrm{ND}^{\mathrm{M}, \mathrm{Ag}}$ & $\mathrm{ND}$ & & $\mathrm{ND}^{\mathrm{Ag}}$ & Underwood et al., 2010 \\
\hline $\mathrm{W} 72^{\mathrm{ECD} \dagger}$ & & $\mathrm{ND}^{\mathrm{C}, \mathrm{Ag}}$ & & & $\begin{array}{l}\text { Membrane expression } \\
\text { confirmed via WB }\end{array}$ & Wilmen et al., 1997 \\
\hline $\mathrm{N} 82^{\mathrm{ECD}}$ & $\mathrm{L}$ & $\mathrm{WT}^{\mathrm{C}, \mathrm{Ag}}$ & WT & $\mathrm{WT}^{\mathrm{Ag}}$ & & Chen et al., $2010 \mathrm{a}$ \\
\hline $\mathrm{W} 87^{\mathrm{ECD}}$ & & $\mathrm{WT}^{\mathrm{C}, \mathrm{Ag}}$ & WT & $24-62 \%$ & $\begin{array}{l}\text { Transient and stable cell } \\
\text { lines analyzed }\end{array}$ & Wilmen et al., 1997 \\
\hline$Y 88^{\mathrm{ECD}}$ & $\mathrm{A}$ & $\mathrm{ND}^{\mathrm{M}, \mathrm{Ag}}$ & ND & & $\mathrm{ND}^{\mathrm{Ag}}$ & Underwood et al., 2010 \\
\hline $\mathrm{L} 89^{\mathrm{ECD}}$ & $\mathrm{A}$ & $\mathrm{ND}^{\mathrm{M}, \mathrm{Ag}}$ & $\mathrm{ND}$ & & $\mathrm{ND}^{\mathrm{Ag}}$ & Underwood et al., 2010 \\
\hline Pro- $90^{\text {ECD }}$ & $\mathrm{A}$ & $\mathrm{WT}^{\mathrm{M}, \mathrm{Ag}}$ & WT & $\mathrm{WT}^{\mathrm{Ag}}$ & & Underwood et al., 2010 \\
\hline $\mathrm{W} 91^{\mathrm{ECD} \dagger}$ & & $\mathrm{ND}^{\mathrm{C}, \mathrm{Ag}}$ & & & $\begin{array}{l}\text { Membrane expression } \\
\text { confirmed via WB }\end{array}$ & Wilmen et al., 1997 \\
\hline $\mathrm{W} 110^{\mathrm{ECD} \dagger}$ & & $\mathrm{ND}^{\mathrm{C}, \mathrm{Ag}}$ & & & $\begin{array}{l}\text { Membrane expression } \\
\text { confirmed via WB }\end{array}$ & Wilmen et al., 1997 \\
\hline $\mathrm{N} 115^{\mathrm{ECD}}$ & $\mathrm{L}$ & $\mathrm{WT}^{\mathrm{C}, \mathrm{Ag}}$ & WT & $\mathrm{WT}^{\mathrm{Ag}}$ & & Chen et al., 2010a \\
\hline $\mathrm{W} 120^{\mathrm{ECD} \dagger}$ & & $\mathrm{ND}^{\mathrm{C}, \mathrm{Ag}}$ & & & $\begin{array}{l}\text { Membrane expression } \\
\text { confirmed via WB }\end{array}$ & Wilmen et al., 1997 \\
\hline $\mathrm{R} 121^{\mathrm{ECD}}$ & $\mathrm{A}$ & $\mathrm{WT}^{\mathrm{M}, \mathrm{Ag}}$ & WT & $\mathrm{WT}^{\mathrm{Ag}}$ & & Underwood et al., 2010 \\
\hline $\mathrm{L} 123^{\mathrm{ECD}}$ & $\mathrm{A}$ & $\mathrm{WT}^{\mathrm{M}, \mathrm{Ag}}$ & WT & $47 \% \mathrm{Ag}$ & & Underwood et al., 2010 \\
\hline $\mathrm{E} 127^{\mathrm{ECD}}$ & $\mathrm{A}$ & $\mathrm{WT}^{\mathrm{M}, \mathrm{Ag}}$ & WT & $\mathrm{WT}^{\mathrm{Ag}}$ & & Underwood et al., 2010 \\
\hline $\mathrm{E} 127^{\mathrm{ECD}}$ & $\mathrm{A}$ & & WT & & & Patterson et al., 2013 \\
\hline $\mathrm{E} 127^{\mathrm{ECD}}$ & $\mathrm{E}$ & $\mathrm{WT}^{\mathrm{M}, \mathrm{Ag}}$ & WT & $\mathrm{WT}^{\mathrm{Ag}}$ & & Underwood et al., 2010 \\
\hline $\mathrm{E} 128^{\mathrm{ECD}}$ & $\mathrm{A}$ & $\mathrm{WT}^{\mathrm{M}, \mathrm{Ag}}$ & WT & $\mathrm{WT}^{\mathrm{Ag}}$ & & Underwood et al., 2010 \\
\hline $\mathrm{E} 128^{\mathrm{ECD}}$ & $\mathrm{A}$ & & WT & & & Patterson et al., 2013 \\
\hline $\mathrm{E} 128^{\mathrm{ECD}}$ & $\mathrm{Q}$ & $\mathrm{WT}^{\mathrm{M}, \mathrm{Ag}}$ & WT & $\mathrm{WT}^{\mathrm{Ag}}$ & & Underwood et al., 2010 \\
\hline $\mathrm{E} 128^{\mathrm{ECD}}$ & $\mathrm{M}$ & & WT & & & Day et al., 2011 \\
\hline $\mathrm{R} 131^{1.26 \mathrm{~b}}$ & $\mathrm{~N}$ & $\mathrm{WT}^{\mathrm{C}, \mathrm{Ant}}$ & WT & $\mathrm{WT}^{\mathrm{E}}$ & & Koole et al., 2011 \\
\hline $\mathrm{L} 141^{1.36 \mathrm{~b}}$ & A & $\mathrm{WT}^{\mathrm{C}, \mathrm{Ag}}$ & WT & $67 \%$ cyt & & Yang et al., 2016 \\
\hline $\mathrm{Y} 145^{1.40 b}$ & $\mathrm{~A}$ & $\mathrm{WT}^{\mathrm{C}, \mathrm{Ag}}$ & WT & $47 \%$ cyt & & Yang et al., 2016 \\
\hline $\mathrm{Y} 148^{1.43 b}$ & $\mathrm{~A}$ & $\mathrm{ND}^{\mathrm{C}, \mathrm{Ag}}$ & 26 & $\mathrm{WT}^{\mathrm{cyt}}$ & & Yang et al., 2016 \\
\hline $\mathrm{Y} 148^{1.43 b}$ & $\mathrm{~N}$ & $15^{\mathrm{C}, \mathrm{Ag}}$ & 8 & $\mathrm{WT}^{\text {cyt }}$ & & Yang et al., 2016 \\
\hline $\mathrm{Y} 148^{1.43 b}$ & $\mathrm{~F}$ & $\mathrm{ND}^{\mathrm{C}, \mathrm{Ag}}$ & 14 & $\mathrm{WT}^{\mathrm{cyt}}$ & & Yang et al., 2016 \\
\hline Y148 $1.43 b$ & $\mathrm{~F}$ & $10^{\mathrm{C}, \mathrm{Ant}}$ & 14 & $\mathrm{WT}^{\mathrm{cyt}}$ & & Yang et al., 2016 \\
\hline $\mathrm{T} 149^{1.44 b}$ & $\mathrm{M}$ & $60^{\mathrm{C}, \mathrm{Ant}}$ & $14-33$ & $\mathrm{WT}^{\mathrm{E} \& \text { Ant }}$ & & Beinborn et al., 2005 \\
\hline $\mathrm{T} 149^{1.44 \mathrm{~b}}$ & $\mathrm{M}^{*}$ & $250^{\mathrm{C}, \text { Ant }}$ & 160 & $<50 \%{ }^{\mathrm{E}}$ & $\begin{array}{l}\mathrm{E}_{\max }=\mathrm{ND} \\
\text { For additional residue } \\
\text { substiutions, see Koole } \\
\text { et al., } 2015\end{array}$ & Koole et al., 2011 \\
\hline $\mathrm{T} 149^{1.43 b}$ & $\mathrm{M}$ & $\mathrm{ND}^{\mathrm{C}, \mathrm{Ag}}$ & 59 & $\mathrm{WT}^{\mathrm{cyt}}$ & & Yang et al., 2016 \\
\hline $\mathrm{T} 149^{1.43 \mathrm{~b}}$ & $\mathrm{~A}$ & $\mathrm{ND}^{\mathrm{C}, \mathrm{Ag}}$ & 82 & $\mathrm{WT}^{\mathrm{cyt}}$ & & Yang et al., 2016 \\
\hline $\mathrm{T} 149^{1.43 \mathrm{~b}}$ & $\mathrm{~S}$ & $\mathrm{WT}^{\mathrm{C}, \mathrm{Ag}}$ & WT & $\mathrm{WT}^{\mathrm{cyt}}$ & & Yang et al., 2016 \\
\hline $\mathrm{Y} 152^{1.47 \mathrm{~b}}$ & $\mathrm{~A}$ & $30^{\mathrm{M}, \mathrm{Ant}}$ & ND & $7 \%$ Ant & & Coopman et al., 2011 \\
\hline $\mathrm{Y} 152^{1.47 \mathrm{~b}}$ & $\mathrm{H}$ & $\mathrm{WT}^{\mathrm{C}, \mathrm{Ag}}$ & WT & $\mathrm{WT}^{\mathrm{cyt}}$ & & Yang et al., 2016 \\
\hline $\mathrm{S} 155^{1.50 \mathrm{~b}}$ & $\mathrm{~A}$ & $\mathrm{WT}^{\mathrm{C}, \mathrm{Ant}}$ & 10 & $55 \%{ }^{\mathrm{E}}, 48 \%^{\mathrm{Ant}}$ & $\Delta \log \tau_{c}=0.75$ & Wootten et al., 2013c \\
\hline $\mathrm{G} 168^{\mathrm{ICL} 1}$ & $\mathrm{~S}$ & $\mathrm{WT}^{\mathrm{C}, \mathrm{Ant}}$ & WT & $<50 \%{ }^{\mathrm{E}}$ & & Koole et al., 2011 \\
\hline F169 & $\mathrm{A}$ & $\mathrm{WT}^{\mathrm{C}, \mathrm{Ag}}$ & & $\mathrm{WT}^{\mathrm{Ag}}$ & WT cAMP $10^{-7}$ M GLP-1 & Mathi et al., 1997 \\
\hline $\mathrm{R} 170^{\mathrm{ICL} 1 \dagger}$ & $\mathrm{A}$ & $\mathrm{WT}^{\mathrm{C}, \mathrm{Ag}}$ & & $\mathrm{WT}^{\mathrm{Ag}}$ & WT cAMP $10^{-7}$ M GLP-1 & Mathi et al., 1997 \\
\hline
\end{tabular}

rat) - the expected lack of effect on GLP-1

pharmacology was implied in text, but no data are shown

Membrane expression confirmed via WB

\section{Membrane expression}

ransient and stable cell alyzed $\mathrm{ND}^{\mathrm{Ag}}$

embrane expression confirmed via WB

embrane expression

embrane expression

$\mathrm{WT}^{\mathrm{Ag}}$

$\mathrm{WT}^{\mathrm{Ag}}$

$\mathrm{WT}^{\mathrm{Ag}}$

$\mathrm{WT}^{\mathrm{cy}}$

$\mathrm{WT}^{\mathrm{cy}}$

WT

$\mathrm{WT}^{\mathrm{E} \& \text { Ant }}$

T149 1.44

$\mathrm{T} 149^{1.44 \mathrm{~b}}$

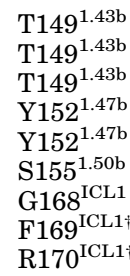

$\mathrm{WT}^{\mathrm{c}}$

$7 \%$ Ant

$\%^{\mathrm{E}}, 48 \%$ Ant $\quad \Delta \log \tau_{\mathrm{c}}=0.75$ $\mathrm{WT}^{\mathrm{Ag}} \quad$ WT cAMP $10^{-7}$ M GLP-1 
TABLE 1-Continued

\begin{tabular}{|c|c|c|c|c|c|c|}
\hline Residue & $\begin{array}{l}\text { Mutated } \\
\text { to }\end{array}$ & $\begin{array}{l}\text {-Fold Reduction } \\
\text { Affinity }\end{array}$ & $\begin{array}{l}\text {-Fold Reduction } \\
\text { Potency }\end{array}$ & $\begin{array}{l}\text { Cell Surface } \\
\text { Expression } \\
(\% \text { Wild-Type })\end{array}$ & $\begin{array}{c}\text { Comments and/or Other } \\
\text { Observed Effects }\end{array}$ & Reference \\
\hline $\mathrm{H} 171^{\mathrm{ICL} 1 \dagger}$ & $\mathrm{A}$ & $\mathrm{WT}^{\mathrm{C}, \mathrm{Ag}}$ & & $\mathrm{WT}^{\mathrm{Ag}}$ & WT cAMP $10^{-7}$ M GLP-1 & Mathi et al., 1997 \\
\hline $\mathrm{L} 172^{\mathrm{ICL} 1 \dagger}$ & $\mathrm{A}$ & $\mathrm{WT}^{\mathrm{C}, \mathrm{Ag}}$ & & $35 \% \mathrm{Ag}$ & WT cAMP $10^{-7}$ M GLP-1 & Mathi et al., 1997 \\
\hline $\mathrm{H} 173^{\mathrm{ICL} 1 \dagger}$ & $\mathrm{A}$ & $\mathrm{WT}^{\mathrm{C}, \mathrm{Ag}}$ & & $48 \% \mathrm{Ag}$ & WT cAMP $10^{-7}$ M GLP-1 & Mathi et al., 1997 \\
\hline $\mathrm{C} 174^{\mathrm{ICL} 1 \dagger}$ & $\mathrm{A}$ & $\mathrm{WT}^{\mathrm{C}, \mathrm{Ag}}$ & & $45 \% \mathrm{Ag}$ & $37 \%$ cAMP $10^{-7}$ M GLP-1 & Mathi et al., 1997 \\
\hline $\mathrm{C} 174^{\mathrm{ICL} 1}$ & $\mathrm{~A}$ & & 6 & & & Underwood et al., 2013 \\
\hline $\mathrm{C} 174^{\mathrm{ICL} 1}$ & $\mathrm{~S}$ & & 7 & & & Underwood et al., 2013 \\
\hline $\mathrm{T} 175^{2.45 \mathrm{~b} \dagger}$ & $\mathrm{A}$ & $\mathrm{WT}^{\mathrm{C}, \mathrm{Ag}}$ & & $57 \% \mathrm{Ag}$ & WT cAMP $10^{-7}$ M GLP-1 & Mathi et al., 1997 \\
\hline $\mathrm{R} 176^{2.46 \mathrm{~b} \dagger}$ & $\mathrm{A}$ & $\mathrm{WT}^{\mathrm{C}, \mathrm{Ag}}$ & 13 & $\mathrm{WT}^{\mathrm{Ag}}$ & $26 \%$ cAMP $10^{-7}$ M GLP-1 & Mathi et al., 1997 \\
\hline $\mathrm{N} 177^{2.47 \mathrm{~b} \dagger}$ & $\mathrm{A}$ & $\mathrm{WT}^{\mathrm{C}, \mathrm{Ag}}$ & & $22 \% \mathrm{Ag}$ & $43 \%$ cAMP $10^{-7}$ M GLP-1 & Mathi et al., 1997 \\
\hline $\mathrm{H} 180^{2.50 \mathrm{~b} \dagger}$ & $\mathrm{R}$ & $21^{\mathrm{C}, \mathrm{Ag}}$ & & $\mathrm{WT}^{\mathrm{mic}}$ & & Heller et al., 1996 \\
\hline $\mathrm{H} 180^{2.50 b}$ & $\mathrm{~A}$ & $\mathrm{ND}^{\mathrm{C}, \mathrm{Ant}}$ & 12 & $18 \%^{\mathrm{E}}, \mathrm{ND}^{\mathrm{Ant}}$ & $\Delta \log \tau_{\mathrm{c}}=0.86$ & Wootten et al., 2013c \\
\hline $\mathrm{N} 182^{2.52 \mathrm{~b}_{\dagger}^{\dagger}}$ & $\mathrm{A}$ & $\mathrm{WT}^{\mathrm{C}, \mathrm{Ag}}$ & & $24 \% \mathrm{Ag}$ & "36\% of WT" cAMP & Xiao et al., 2000 \\
\hline $\mathrm{S} 186^{2.56 \mathrm{~b}}$ & $\mathrm{~A}$ & $\mathrm{WT}^{\mathrm{C}, \mathrm{Ant}}$ & WT & $\mathrm{WT}^{\mathrm{E}, \mathrm{Ant}}$ & & Wootten et al., 2013c \\
\hline $\mathrm{F} 187^{2.57 \mathrm{~b}}$ & $\mathrm{~A}$ & $\mathrm{ND}^{\mathrm{C}, \mathrm{Ag}}$ & ND & $5 \%$ cyt & & Yang et al., 2016 \\
\hline $\mathrm{R} 190^{2.60 \mathrm{~b} \dagger}$ & $\mathrm{A}$ & $>20^{\mathrm{C}, \mathrm{Ag}}$ & & $21 \%{ }^{\mathrm{Ag}}$ & “27\% of WT" cAMP & Xiao et al., 2000 \\
\hline $\mathrm{R} 190^{2.60 \mathrm{~b}}$ & $\mathrm{~A}$ & $32^{\mathrm{M}, \mathrm{Ant}}$ & 270 & $7 \%$ Ant & & Coopman et al., 2011 \\
\hline $\mathrm{R} 190^{2.60 \mathrm{~b}}$ & $\mathrm{~A}$ & $20^{\text {C,Ant }}$ & 34 & $53 \%^{\mathrm{E}}, 44^{\text {Ant }}$ & $\Delta \log \tau_{c}=0.53$ & Wootten et al., 2013c \\
\hline $\mathrm{R} 190^{2.60 \mathrm{~b}}$ & $\mathrm{~A}$ & $\mathrm{ND}^{\mathrm{C}, \mathrm{Ag}}$ & ND & $\mathrm{WT}^{\mathrm{cyt}}$ & & Yang et al., 2016 \\
\hline $\mathrm{R} 190^{2.60 \mathrm{~b}}$ & $\mathrm{~K}$ & $\mathrm{ND}^{\mathrm{C}, \mathrm{Ag}}$ & 17 & $\mathrm{WT}^{\mathrm{cyt}}$ & & Yang et al., 2016 \\
\hline $\mathrm{R} 190^{2.60 \mathrm{~b}}$ & $\mathrm{~K}$ & $32^{\mathrm{C}, \mathrm{Ant}}$ & 17 & $\mathrm{WT}^{\mathrm{cyt}}$ & & Yang et al., 2016 \\
\hline $\mathrm{L} 192^{2.62 b}$ & $\mathrm{~S}$ & & WT & & & Underwood et al., 2011 \\
\hline $\mathrm{L} 192^{2.62 b}$ & $\mathrm{~S}$ & $\mathrm{WT}^{\mathrm{C}, \mathrm{Ag}}$ & WT & $\mathrm{WT}^{\mathrm{cyt}}$ & & Yang et al., 2016 \\
\hline V194 $2.64 \mathrm{~b}$ & $\mathrm{~A}$ & $\mathrm{WT}^{\mathrm{C}, \mathrm{Ag}}$ & WT & $\mathrm{WT}^{\text {cyt }}$ & & Yang et al., 2016 \\
\hline F195 & $\mathrm{L}$ & & WT & $59 \% \mathrm{Ag}$ & & Underwood et al., 2011 \\
\hline $\mathrm{I} 196^{2.66 \mathrm{~b} \dagger}$ & $\mathrm{S}$ & $\mathrm{WT}^{\mathrm{C}, \mathrm{Ag}}$ & ND & & & Moon et al., 2012 \\
\hline $\mathrm{K} 197^{2.67 \mathrm{~b}}$ & $\mathrm{~A}$ & $28^{\mathrm{M}, \mathrm{Ant}}$ & 630 & $57 \%$ Ant & & Coopman et al., 2011 \\
\hline $\mathrm{K} 197^{2.67 \mathrm{~b} \dagger}$ & $\mathrm{A}$ & $5^{\mathrm{C}, \mathrm{Ag}}$ & & $30 \% \mathrm{Ag}$ & " $25 \%$ of WT" cAMP & Xiao et al., 2000 \\
\hline $\mathrm{K} 197^{2.67 \mathrm{~b}}$ & $\mathrm{~A}$ & $\mathrm{ND}^{\mathrm{C}, \mathrm{Ag}}$ & ND & $51 \%{ }^{\mathrm{cyt}}$ & & Yang et al., 2016 \\
\hline $\mathrm{K} 197^{2.67 \mathrm{~b}}$ & $\mathrm{I}$ & $\mathrm{ND}^{\mathrm{C}, \mathrm{Ag}}$ & ND & $51 \%$ cyt & & Yang et al., 2016 \\
\hline $\mathrm{K} 197^{2.67 \mathrm{~b}}$ & $\mathrm{I}$ & $28^{\mathrm{C}, \mathrm{Ant}}$ & $\mathrm{ND}$ & $51 \%{ }^{\mathrm{cyt}}$ & & Yang et al., 2016 \\
\hline $\mathrm{K} 197^{2.67 \mathrm{~b}}$ & $\mathrm{Q}$ & $\mathrm{ND}^{\mathrm{C}, \mathrm{Ag}}$ & ND & $\mathrm{WT}^{\mathrm{cyt}}$ & & Yang et al., 2016 \\
\hline $\mathrm{K} 197^{2.67 \mathrm{~b}}$ & $\mathrm{R}$ & $\mathrm{ND}^{\mathrm{C}, \mathrm{Ag}}$ & 23 & $\mathrm{WT}^{\mathrm{cyt}}$ & & Yang et al., 2016 \\
\hline $\mathrm{D} 198^{2.68 \mathrm{~b} \dagger}$ & A & $10^{\mathrm{C}, \mathrm{Ag}}$ & & $16 \% \mathrm{Ag}$ & " $45 \%$ of WT" cAMP & Xiao et al., 2000 \\
\hline $\mathrm{D} 198^{2.68 \mathrm{~b} \dagger}$ & $\mathrm{A}$ & $63^{\mathrm{M}, \mathrm{Ant}}$ & 44 & $\mathrm{WT}^{\mathrm{Ant}}$ & & $\begin{array}{l}\text { López de Maturana and } \\
\text { Donnelly, } 2002\end{array}$ \\
\hline $\mathrm{D} 198^{2.68 \mathrm{~b}}$ & $\mathrm{~A}$ & $43^{\mathrm{M}, \mathrm{Ant}}$ & 977 & $66 \%$ Ant & & Coopman et al., 2011 \\
\hline $\mathrm{D} 198^{2.68 \mathrm{~b}}$ & $\mathrm{~A}$ & $\mathrm{ND}^{\mathrm{C}, \mathrm{Ag}}$ & ND & $\mathrm{WT}^{\mathrm{cyt}}$ & & Yang et al., 2016 \\
\hline $\mathrm{D} 198^{2.68 \mathrm{~b} \dagger}$ & $\mathrm{N}$ & $\mathrm{WT}^{\mathrm{M}, \mathrm{Ag}}$ & & & & $\begin{array}{l}\text { López de Maturana and } \\
\text { Donnelly, } 2002\end{array}$ \\
\hline $\mathrm{D} 198^{2.68 \mathrm{~b}}$ & $\mathrm{~N}$ & $\mathrm{ND}^{\mathrm{C}, \mathrm{Ag}}$ & 89 & $\mathrm{WT}^{\mathrm{cyt}}$ & & Yang et al., 2016 \\
\hline $\mathrm{D} 198^{2.68 \mathrm{~b}}$ & $\mathrm{E}$ & $\mathrm{ND}^{\mathrm{C}, \mathrm{Ag}}$ & 434 & $\mathrm{WT}^{\mathrm{cyt}}$ & & Yang et al., 2016 \\
\hline $\mathrm{D} 198^{2.68 b}$ & $\mathrm{E}$ & $58^{\mathrm{C}, \mathrm{Ant}}$ & 434 & $\mathrm{WT}^{\mathrm{cyt}}$ & & Yang et al., 2016 \\
\hline A200-L201 & V/A & $\mathrm{WT}^{\mathrm{M}, \mathrm{Ag}}$ & WT & & & López de Maturana et al., 2004 \\
\hline $\mathrm{L} 201^{2.68 \mathrm{~b}}$ & $\mathrm{~A}$ & $\mathrm{ND}^{\mathrm{C}, \mathrm{Ag}}$ & ND & $\mathrm{WT}^{\mathrm{cyt}}$ & & Yang et al., 2016 \\
\hline $\mathrm{K} 202^{\mathrm{ECL} 1 \dagger}$ & $\mathrm{A}$ & $\mathrm{WT}^{\mathrm{C}, \mathrm{Ag}}$ & & $45 \% \mathrm{Ag}$ & "71\% of WT" cAMP & Xiao et al., 2000 \\
\hline $\mathrm{K} 202 / \mathrm{W} 203^{\dagger}$ & $\mathrm{A} / \mathrm{A}$ & $\mathrm{WT}^{\mathrm{M}, \mathrm{Ag}}$ & WT & & & López de Maturana et al., 2004 \\
\hline $\mathrm{W} 203^{\mathrm{ECL} 1}$ & $\mathrm{~T}$ & $\mathrm{WT}^{\mathrm{C}, \mathrm{Ag}}$ & WT & $\mathrm{WT}^{\mathrm{cyt}}$ & & Yang et al., 2016 \\
\hline $\mathrm{M} 204 / \mathrm{Y}_{205^{\dagger}}$ & $\mathrm{A} / \mathrm{A}$ & $37^{\mathrm{M}, \mathrm{Ant}}$ & 51 & $28 \%$ Ant & & López de Maturana et al., 2004 \\
\hline $\mathrm{M} 204 / \mathrm{Y}^{2} 25^{\dagger}$ & V/A & $23^{\mathrm{M}, \mathrm{Ag}}$ & 32 & $\mathrm{WT}^{\mathrm{Ant}}$ & & López de Maturana et al., 2004 \\
\hline M204/Y205 & $\mathrm{A} / \mathrm{V}$ & $29^{\mathrm{M}, \mathrm{Ag}}$ & 87 & $\mathrm{WT}^{\mathrm{Ant}}$ & & López de Maturana et al., 2004 \\
\hline $\mathrm{M} 204^{\mathrm{ECL} 1 \dagger}$ & $\mathrm{A}$ & $\mathrm{WT}^{\mathrm{M}, \mathrm{Ag}}$ & WT & $44 \%$ Ant & & López de Maturana et al., 2004 \\
\hline $\mathrm{M} 204^{\mathrm{ECL} 1}$ & $\mathrm{~A}$ & $\mathrm{ND}^{\mathrm{C}, \mathrm{Ag}}$ & 334 & $\mathrm{WT}^{\mathrm{cyt}}$ & & Yang et al., 2016 \\
\hline $\mathrm{M} 204^{\mathrm{ECL} 1}$ & $\mathrm{R}$ & $\mathrm{ND}^{\mathrm{C}, \mathrm{Ag}}$ & 93 & $\mathrm{WT}^{\text {cyt }}$ & & Yang et al., 2016 \\
\hline Y $205^{\text {ECL } 1 \dagger}$ & $\mathrm{A}$ & $\mathrm{WT}^{\mathrm{M}, \mathrm{Ag}}$ & WT & $\mathrm{WT}^{\text {Ant }}$ & & López de Maturana et al., 2004 \\
\hline $\mathrm{Y} 205^{\mathrm{ECL} 1}$ & $\mathrm{~A}$ & $\mathrm{ND}^{\mathrm{C}, \mathrm{Ag}}$ & 62 & $\mathrm{WT}^{\mathrm{cyt}}$ & & Yang et al., 2016 \\
\hline $\mathrm{S} 206 / \mathrm{T} 207^{\dagger}$ & A/A & $\mathrm{WT}^{\mathrm{M}, \mathrm{Ag}}$ & WT & & & López de Maturana et al., 2004 \\
\hline A208/A209 & $\mathrm{V} / \mathrm{V}$ & $\mathrm{WT}^{\mathrm{M}, \mathrm{Ag}}$ & WT & & & López de Maturana et al., 2004 \\
\hline $\mathrm{Q} 210 \mathrm{Q} 211^{\dagger}$ & $\mathrm{A} / \mathrm{A}$ & $\mathrm{WT}^{\mathrm{M}, \mathrm{Ag}}$ & WT & & & López de Maturana et al., 2004 \\
\hline $\mathrm{Q} 211^{\mathrm{ECL} 1}$ & $\mathrm{D}$ & $\mathrm{WT}^{\mathrm{C}, \mathrm{Ag}}$ & WT & $\mathrm{WT}^{\mathrm{cyt}}$ & & Yang et al., 2016 \\
\hline $\mathrm{Q} 211^{\mathrm{ECL} 1}$ & $\mathrm{R}$ & $\mathrm{WT}^{\mathrm{C}, \mathrm{Ag}}$ & WT & $\mathrm{WT}^{\mathrm{cyt}}$ & & Yang et al., 2016 \\
\hline $\mathrm{H} 212^{\mathrm{ECL} 1 \dagger}$ & $\mathrm{A}$ & $\mathrm{WT}^{\mathrm{C}, \mathrm{Ag}}$ & & $\mathrm{WT}^{\mathrm{Ag}}$ & "WT cAMP" & Xiao et al., 2000 \\
\hline $\mathrm{H} 212^{\mathrm{ECL} 1}$ & $\mathrm{~A}$ & $\mathrm{WT}^{\mathrm{C}, \mathrm{Ag}}$ & WT & $\mathrm{WT}^{\mathrm{cyt}}$ & & Yang et al., 2016 \\
\hline $\mathrm{H} 212 / \mathrm{Q} 213^{\dagger}$ & $\mathrm{A} / \mathrm{A}$ & $\mathrm{WT}^{\mathrm{M}, \mathrm{Ag}}$ & WT & & & López de Maturana et al., 2004 \\
\hline $\mathrm{D} 215^{\mathrm{ECL} 1 \dagger}$ & $\mathrm{A}$ & $\mathrm{WT}^{\mathrm{C}, \mathrm{Ag}}$ & & $51 \% \mathrm{Ag}$ & "57\% of WT" cAMP & Xiao et al., 2000 \\
\hline $\mathrm{W} 214^{\mathrm{ECL} 1}$ & $\mathrm{~V}$ & $\mathrm{WT}^{\mathrm{C}, \mathrm{Ag}}$ & WT & $\mathrm{WT}^{\text {cyt }}$ & & Yang et al., 2016 \\
\hline $\mathrm{W} 214 / \mathrm{D} 215^{\dagger}$ & $\mathrm{A} / \mathrm{A}$ & $\mathrm{WT}^{\mathrm{M}, \mathrm{Ag}}$ & WT & & & López de Maturana et al., 2004 \\
\hline G216/L217 & $\mathrm{A} / \mathrm{A}$ & $\mathrm{WT}^{\mathrm{M}, \mathrm{Ag}}$ & WT & & & López de Maturana et al., 2004 \\
\hline $\mathrm{L} 217^{\mathrm{ECL} 1}$ & $\mathrm{~A}$ & $\mathrm{WT}^{\mathrm{C}, \mathrm{Ag}}$ & WT & $\mathrm{WT}^{\mathrm{cyt}}$ & & Yang et al., 2016 \\
\hline $\mathrm{L} 218 / \mathrm{S} 219^{\dagger}$ & $\mathrm{A} / \mathrm{A}$ & $\mathrm{WT}^{\mathrm{M}, \mathrm{Ag}}$ & WT & & & López de Maturana et al., 2004 \\
\hline $\mathrm{Y} 220 / \mathrm{Q} 221^{\dagger}$ & $\mathrm{A} / \mathrm{A}$ & $\mathrm{WT}^{\mathrm{M}, \mathrm{Ag}}$ & WT & & & López de Maturana et al., 2004 \\
\hline $\mathrm{Y} 220^{\mathrm{ECL} 1}$ & $\mathrm{D}$ & $\mathrm{ND}^{\mathrm{C}, \mathrm{Ag}}$ & 105 & $\mathrm{WT}^{\mathrm{cyt}}$ & & Yang et al., 2016 \\
\hline $\mathrm{D} 222^{3.25 \mathrm{~b} \dagger}$ & $\mathrm{A}$ & $\mathrm{WT}^{\mathrm{C}, \mathrm{Ag}}$ & & $\mathrm{WT}^{\mathrm{Ag}}$ & " $82 \%$ of WT" cAMP & Xiao et al., 2000 \\
\hline $\mathrm{D} 222 / \mathrm{S} 223^{\dagger}$ & $\mathrm{A} / \mathrm{A}$ & $\mathrm{WT}^{\mathrm{M}, \mathrm{Ag}}$ & WT & & & López de Maturana et al., 2004 \\
\hline
\end{tabular}


TABLE 1-Continued

\begin{tabular}{|c|c|c|c|c|c|c|}
\hline Residue & $\begin{array}{l}\text { Mutated } \\
\text { to }\end{array}$ & $\begin{array}{l}\text {-Fold Reduction } \\
\text { Affinity }\end{array}$ & $\begin{array}{l}\text {-Fold Reduction } \\
\text { Potency }^{*}\end{array}$ & $\begin{array}{c}\text { Cell Surface } \\
\text { Expression } \\
\text { (\% Wild-Type) }\end{array}$ & $\begin{array}{l}\text { Comments and/or Other } \\
\text { Observed Effects }\end{array}$ & Reference \\
\hline $\mathrm{L} 224 / \mathrm{G} 225^{\dagger}$ & $\mathrm{A} / \mathrm{A}$ & $\mathrm{WT}^{\mathrm{M}, \mathrm{Ag}}$ & WT & & & López de Maturana et al., 2004 \\
\hline $\mathrm{C} 226^{3.29 \mathrm{~b} * \dagger}$ & A & $25^{\mathrm{M}, \mathrm{Ant}}$ & 38 & $65 \%$ Ant & & Mann et al., $2010 \mathrm{a}$ \\
\hline $\mathrm{C} 226^{3.29 \mathrm{~b}}$ & $\mathrm{~A}$ & & $>90$ & & & Underwood et al., 2013 \\
\hline $\mathrm{R} 227^{3.30 \mathrm{~b} \dagger}$ & $\mathrm{A}$ & $>20^{\mathrm{C}, \mathrm{Ag}}$ & & $31 \%{ }^{\mathrm{Ag}}$ & “90\% WT" cAMP & Xiao et al., 2000 \\
\hline $\mathrm{R} 227 / \mathrm{L} 228^{\dagger}$ & $\mathrm{A} / \mathrm{A}$ & $\mathrm{WT}^{\mathrm{M}, \mathrm{Ag}}$ & WT & & & López de Maturana et al., 2004 \\
\hline $\mathrm{V} 229 / \mathrm{F} 230^{\dagger}$ & $\mathrm{A} / \mathrm{A}$ & $\mathrm{WT}^{\mathrm{M}, \mathrm{Ag}}$ & WT & & & López de Maturana et al., 2004 \\
\hline $\mathrm{L} 232 / \mathrm{M} 233^{\dagger}$ & $\mathrm{V} / \mathrm{T}$ & $10^{\mathrm{C}, \mathrm{Ag}}$ & 100 & & & Moon et al., 2012 \\
\hline $\mathrm{M} 233^{3.36 \mathrm{~b}}$ & $\mathrm{~A}$ & $\mathrm{ND}^{\mathrm{C}, \mathrm{Ag}}$ & 70 & $\mathrm{WT}^{\mathrm{cyt}}$ & & Yang et al., 2016 \\
\hline $\mathrm{M} 233^{3.36 \mathrm{~b}}$ & $\mathrm{~A}$ & $16^{\mathrm{C}, \mathrm{Ant}}$ & 70 & $\mathrm{WT}^{\mathrm{cyt}}$ & & Yang et al., 2016 \\
\hline $\mathrm{M} 233^{3.36 \mathrm{~b}}$ & $\mathrm{~T}$ & $\mathrm{ND}^{\mathrm{C}, \mathrm{Ag}}$ & 62 & $\mathrm{WT}^{\mathrm{cyt}}$ & & Yang et al., 2016 \\
\hline M233 $3.36 \mathrm{~b}$ & $\mathrm{~F}$ & $\mathrm{WT}^{\mathrm{C}, \mathrm{Ag}}$ & WT & $\mathrm{WT}^{\mathrm{cyt}}$ & & Yang et al., 2016 \\
\hline $\mathrm{Q} 234^{3.37 \mathrm{~b}}$ & $\mathrm{~A}$ & $13^{\mathrm{M}, \text { Ant }}$ & 45 & $27 \%$ Ant & & Coopman et al., 2011 \\
\hline $\mathrm{Q} 234^{3.37 \mathrm{~b}}$ & A & $\mathrm{ND}^{\mathrm{C}, \mathrm{Ag}}$ & 151 & $35 \%$ cyt & & Yang et al., 2016 \\
\hline $\mathrm{Q} 234^{3.37 \mathrm{~b}}$ & $\mathrm{~N}$ & $\mathrm{WT}^{\mathrm{C}, \mathrm{Ag}}$ & WT & $\mathrm{WT}^{\mathrm{cyt}}$ & & Yang et al., 2016 \\
\hline $\mathrm{Q} 234^{3.37 \mathrm{~b}}$ & $\mathrm{E}$ & $\mathrm{ND}^{\mathrm{C}, \mathrm{Ag}}$ & 29 & $69 \%^{\text {cyt }}$ & & Yang et al., 2016 \\
\hline $\mathrm{Q} 234^{3.37 \mathrm{~b}}$ & $\mathrm{E}$ & $17^{\mathrm{C}, \mathrm{Ant}}$ & 29 & $69 \%$ cyt & & Yang et al., 2016 \\
\hline Y $235^{3.38 b}$ & $\mathrm{~A}$ & $24^{\mathrm{M}, \text { Ant }}$ & 23 & $12 \%$ Ant & & Coopman et al., 2011 \\
\hline $\mathrm{C} 236^{3.39 \mathrm{~b}}$ & $\mathrm{~A}$ & & WT & & & Underwood et al., 2013 \\
\hline $\mathrm{N} 240^{3.43 \mathrm{~b} \dagger}$ & $\mathrm{A}$ & $>20^{\mathrm{C}, \mathrm{Ag}}$ & & $14 \% \mathrm{Ag}$ & "8\% WT" cAMP & Xiao et al., 2000 \\
\hline $\mathrm{N} 240^{3.43 b}$ & A & $\mathrm{WT}^{\mathrm{C}, \mathrm{Ant}}$ & 7 & $\mathrm{WT}^{\mathrm{E}, \mathrm{Ant}}$ & $\Delta \log \tau_{\mathrm{c}}=0.67$ & Wootten et al., 2013c \\
\hline $\mathrm{N} 240^{3.43 \mathrm{~b}}$ & $\mathrm{Q}$ & $\mathrm{WT}^{\mathrm{C}, \mathrm{Ant}}$ & WT & $\mathrm{WT}^{\mathrm{E}, \mathrm{Ant}}$ & & Wootten et al., 2016 \\
\hline N240/Q394 & A/A & $\mathrm{WT}^{\mathrm{C}, \mathrm{Ant}}$ & WT & $71 \%{ }^{\mathrm{E}, \mathrm{Ant}}$ & $\Delta \log \tau_{\mathrm{c}}=0.70$ & Wootten et al., 2016 \\
\hline N240/Q394 & $\mathrm{Q} / \mathrm{N}$ & $5^{\mathrm{C}, \mathrm{Ant}}$ & WT & $71 \%{ }^{\mathrm{E}, \text { Ant }}$ & & Wootten et al., 2016 \\
\hline $\mathrm{Y} 241^{3.44 \mathrm{~b}}$ & A & & WT & $42 \% \mathrm{Ag}$ & & Underwood et al., 2011 \\
\hline $\mathrm{Y} 241^{3.44 \mathrm{~b}}$ & $\mathrm{~A}$ & $\mathrm{WT}^{\mathrm{C}, \mathrm{Ag}}$ & WT & $\mathrm{WT}^{\mathrm{cyt}}$ & & Yang et al., 2016 \\
\hline $\mathrm{E} 247^{3.50 \mathrm{~b}}$ & $\mathrm{~A}$ & $\mathrm{WT}^{\mathrm{C}, \mathrm{Ag}}$ & & $64 \% \mathrm{Ag}$ & “39\% WT" cAMP & Xiao et al., 2000 \\
\hline $\mathrm{E} 247^{3.50 \mathrm{~b}}$ & $\mathrm{~A}$ & $\mathrm{ND}^{\mathrm{C}, \mathrm{Ant}}$ & 14 & $18 \%{ }^{\mathrm{E}}, \mathrm{ND}^{\mathrm{Ant}}$ & $\Delta \log \tau_{\mathrm{c}}=0.99$ & Wootten et al., 2013c \\
\hline F260 ${ }^{\text {ICL2 }}$ & $\mathrm{L}$ & $\mathrm{WT}^{\mathrm{C}, \mathrm{Ant}}$ & WT & $<50 \%{ }^{\mathrm{E}}$ & & Koole et al., 2011 \\
\hline $\mathrm{E} 262^{\mathrm{ICL} 2 \dagger}$ & $\mathrm{A}$ & $\mathrm{WT}^{\mathrm{C}, \mathrm{Ag}}$ & & $\mathrm{WT}^{\mathrm{Ag}}$ & WT cAMP $10^{-7}$ M GLP-1 & Mathi et al., 1997 \\
\hline $\mathrm{Q} 263^{\mathrm{ICL} 2 \dagger}$ & $\mathrm{A}$ & $\mathrm{WT}^{\mathrm{C}, \mathrm{Ag}}$ & & $\mathrm{WT}^{\mathrm{Ag}}$ & WT cAMP $10^{-7}$ M GLP-1 & Mathi et al., 1997 \\
\hline $\mathrm{R} 264^{\mathrm{ICL} 2 \dagger}$ & $\mathrm{A}$ & $\mathrm{WT}^{\mathrm{C}, \mathrm{Ag}}$ & & $\mathrm{WT}^{\mathrm{Ag}}$ & WT cAMP $10^{-7}$ M GLP-1 & Mathi et al., 1997 \\
\hline $\mathrm{I} 265^{\mathrm{ICL} 2 \dagger}$ & $\mathrm{A}$ & $\mathrm{WT}^{\mathrm{C}, \mathrm{Ag}}$ & & $\mathrm{WT}^{\mathrm{Ag}}$ & WT cAMP $10^{-7}$ M GLP-1 & Mathi et al., 1997 \\
\hline $\mathrm{F} 266^{4.42 \mathrm{~b} \dagger}$ & A & $\mathrm{WT}^{\mathrm{C}, \mathrm{Ag}}$ & & $\mathrm{WT}^{\mathrm{Ag}}$ & WT cAMP $10^{-7}$ M GLP-1 & Mathi et al., 1997 \\
\hline $\mathrm{K} 267^{4.43 \mathrm{~b} \dagger}$ & $\mathrm{A}$ & $\mathrm{WT}^{\mathrm{C}, \mathrm{Ag}}$ & & $\mathrm{WT}^{\mathrm{Ag}}$ & WT cAMP $10^{-7}$ M GLP-1 & Mathi et al., 1997 \\
\hline $\mathrm{L} 268^{4.44 \mathrm{~b} \dagger}$ & $\mathrm{A}$ & $\mathrm{WT}^{\mathrm{C}, \mathrm{Ag}}$ & & $\mathrm{WT}^{\mathrm{Ag}}$ & WT cAMP $10^{-7}$ M GLP-1 & Mathi et al., 1997 \\
\hline $\mathrm{L} 278^{4.54 b}$ & $\mathrm{M}$ & & WT & & & Underwood et al., 2011 \\
\hline $\mathrm{W} 284^{4.60 \mathrm{~b}}$ & $\mathrm{~A}$ & $32^{\mathrm{M}, \mathrm{Ant}}$ & 1349 & $34 \%$ Ant & & Coopman et al., 2011 \\
\hline $\mathrm{G} 285^{4.61 \mathrm{~b}}$ & $\mathrm{~A}$ & $\mathrm{WT}^{\mathrm{C}, \mathrm{Ant}}$ & WT & $\mathrm{WT}^{\mathrm{E}}$ & & Koole et al., $2012 \mathrm{a}$ \\
\hline $\mathrm{I} 286^{4.62 \mathrm{~b}}$ & $\mathrm{~A}$ & $\mathrm{WT}^{\mathrm{C}, \mathrm{Ant}}$ & WT & $\mathrm{WT}^{\mathrm{E}}$ & & Koole et al., $2012 \mathrm{a}$ \\
\hline $\mathrm{V} 287^{4.63 \mathrm{~b}}$ & $\mathrm{~A}$ & $\mathrm{WT}^{\mathrm{C}, \mathrm{Ant}}$ & WT & $\mathrm{WT}^{\mathrm{E}}$ & & Koole et al., $2012 \mathrm{a}$ \\
\hline I286/V287 & $\mathrm{A} / \mathrm{A}$ & & WT & & $85 \% \mathrm{SB}^{\mathrm{M}, \mathrm{Ag}}$ & Dods and Donnelly, 2015 \\
\hline $\mathrm{K} 288^{4.64 \mathrm{~b}}$ & A & $126^{\mathrm{C}, \mathrm{Ant}}$ & ND & $\mathrm{WT}^{\mathrm{E}}$ & & Koole et al., $2012 \mathrm{a}$ \\
\hline $\mathrm{K} 288^{4.64 \mathrm{~b}}$ & $\mathrm{~A}$ & $23^{\mathrm{M}, \text { Ant }}$ & 5732 & $\mathrm{WT}^{\mathrm{Ant}}$ & $\Delta \log \tau_{c}=1.39$ & Dods and Donnelly, 2015 \\
\hline $\mathrm{K} 288^{4.64 \mathrm{~b} \dagger}$ & $\mathrm{A}$ & $79^{\mathrm{M}, \text { Ant }}$ & 251 & & & Al-Sabah and Donnelly, 2003b \\
\hline $\mathrm{K} 288^{4.64 \mathrm{~b} \dagger}$ & $\mathrm{L}$ & $63^{\mathrm{M}, \text { Ant }}$ & 79 & & & Al-Sabah and Donnelly, 2003b \\
\hline $\mathrm{K} 288^{4.64 b}$ & $\mathrm{~L}$ & $\mathrm{ND}^{\mathrm{C}, \mathrm{Ag}}$ & $\mathrm{ND}$ & $70 \%$ cyt & & Yang et al., 2016 \\
\hline $\mathrm{K} 288^{4.64 \mathrm{~b} \dagger}$ & $\mathrm{R}$ & $\mathrm{WT}^{\mathrm{M}, \mathrm{Ag}}$ & WT & & & Al-Sabah and Donnelly, 2003b \\
\hline K288/Y289 & $\mathrm{A}, \mathrm{A}$ & & 2188 & & $28 \% \mathrm{SB}^{\mathrm{M}, \mathrm{Ag}}$ & Dods and Donnelly, 2015 \\
\hline $\mathrm{Y} 289^{4.65 b}$ & A & $\mathrm{WT}^{\mathrm{C}, \mathrm{Ant}}$ & WT & $\mathrm{WT}^{\mathrm{E}}$ & & Koole et al., $2012 \mathrm{a}$ \\
\hline Y289 $4.65 b$ & $\mathrm{~A}$ & $\mathrm{WT}^{\mathrm{M}, \mathrm{Ant}}$ & WT & $20 \%$ Ant & & Dods and Donnelly, 2015 \\
\hline $\mathrm{L} 290^{4.66 \mathrm{~b}}$ & $\mathrm{~A}$ & $\mathrm{WT}^{\mathrm{C}, \mathrm{Ant}}$ & WT & $\mathrm{WT}^{\mathrm{E}}$ & & Koole et al., $2012 \mathrm{a}$ \\
\hline$Y 291^{4.67 b}$ & $\mathrm{~A}$ & $\mathrm{WT}^{\mathrm{C}, \mathrm{Ant}}$ & WT & $\mathrm{WT}^{\mathrm{E}}$ & & Koole et al., $2012 \mathrm{a}$ \\
\hline $\mathrm{L} 290 / \mathrm{Y} 291^{\dagger}$ & $\mathrm{A} / \mathrm{A}$ & $\mathrm{WT}^{\mathrm{M}, \mathrm{Ag}}$ & WT & & & Mann et al., 2010a \\
\hline L290-Y291 & $\mathrm{A} / \mathrm{A}$ & & WT & & $99 \% \mathrm{SB}^{\mathrm{M}, \mathrm{Ag}}$ & Dods and Donnelly, 2015 \\
\hline $\mathrm{E} 292^{\mathrm{ECL} 2}$ & A & $100^{\mathrm{C}, \mathrm{Ant}}$ & 126 & $\mathrm{WT}^{\mathrm{E}}$ & $\Delta \log \tau_{\mathrm{c}}=0.57$ & Koole et al., 2012a \\
\hline $\mathrm{E} 292^{\mathrm{ECL} 2}$ & $\mathrm{~A}$ & $\mathrm{ND}^{\mathrm{C}, \mathrm{Ag}}$ & 33 & $39 \%{ }^{\text {cyt }}$ & & Yang et al., 2016 \\
\hline $\mathrm{D} 293^{\mathrm{ECL} 2}$ & $\mathrm{~A}$ & $25^{\mathrm{C}, \text { Ant }}$ & 16 & $62 \%^{\mathrm{E}}$ & & Koole et al., 2012a \\
\hline $\mathrm{E} 292 / \mathrm{D} 293^{\dagger}$ & A/A & $8^{\mathrm{M}, \mathrm{Ag}}$ & 79 & & & Mann et al., $2010 \mathrm{a}$ \\
\hline E292/D293 & $\mathrm{A} / \mathrm{A}$ & & 25 & & $75 \% \mathrm{SB}^{\mathrm{M}, \mathrm{Ag}}$ & Dods and Donnelly, 2015 \\
\hline $\mathrm{E} 294^{\mathrm{ECL} 2}$ & A & $\mathrm{WT}^{\mathrm{C}, \mathrm{Ant}}$ & WT & $\mathrm{WT}^{\mathrm{E}}$ & $\Delta \log \tau_{\mathrm{c}}=0.65$ & Koole et al., $2012 \mathrm{a}$ \\
\hline $\mathrm{G} 295^{\mathrm{ECL} 2}$ & $\mathrm{~A}$ & $\mathrm{WT}^{\mathrm{C}, \mathrm{Ant}}$ & WT & $\mathrm{WT}^{\mathrm{E}}$ & & Koole et al., 2012a \\
\hline E294-G295 & $\mathrm{A} / \mathrm{A}$ & $\mathrm{WT}^{\mathrm{M}, \mathrm{Ag}}$ & WT & & & Mann et al., 2010a \\
\hline E294-G295 & $\mathrm{A} / \mathrm{A}$ & & WT & & $97 \% \mathrm{SB}^{\mathrm{M}, \mathrm{Ag}}$ & Dods and Donnelly, 2015 \\
\hline $\mathrm{C} 296^{\mathrm{ECL} 2 * \dagger}$ & A & $18^{\mathrm{M}, \text { Ant }}$ & WT & $23 \%$ Ant & & Mann et al., 2010a \\
\hline $\mathrm{C} 296^{\mathrm{ECL} 2}$ & $\mathrm{~A}$ & $13^{\mathrm{C}, \mathrm{Ant}}$ & 126 & $60^{\mathrm{E}}$ & & Koole et al., 2012a \\
\hline $\mathrm{C} 296^{\mathrm{ECL} 2}$ & $\mathrm{~S}$ & $\mathrm{ND}^{\mathrm{C}, \mathrm{Ag}}$ & 96 & $\mathrm{WT}^{\mathrm{cyt}}$ & & Yang et al., 2016 \\
\hline W297 ${ }^{\mathrm{ECL} 2}$ & $\mathrm{~A}$ & $63^{\mathrm{C}, \mathrm{Ant}}$ & 316 & $\mathrm{WT}^{\mathrm{E}}$ & $\Delta \log \tau_{c}=1.00$ & Koole et al., $2012 \mathrm{a}$ \\
\hline $\mathrm{W} 297^{\mathrm{ECL} 2}$ & $\mathrm{~A}$ & $\mathrm{ND}^{\mathrm{C}, \mathrm{Ag}}$ & ND & $\mathrm{WT}^{\mathrm{cyt}}$ & & Yang et al., 2016 \\
\hline W297 ${ }^{\text {ECL2 }}$ & $\mathrm{H}$ & $\mathrm{ND}^{\mathrm{C}, \mathrm{Ag}}$ & 50 & $40 \%$ cyt & & Yang et al., 2016 \\
\hline $\mathrm{T} 298^{\mathrm{ECL} 2}$ & $\mathrm{~A}$ & $\mathrm{WT}^{\mathrm{C}, \mathrm{Ant}}$ & WT & $\mathrm{WT}^{\mathrm{E}}$ & & Koole et al., 2012a \\
\hline $\mathrm{W} 297 / \mathrm{T} 298^{\dagger}$ & $\mathrm{A} / \mathrm{A}$ & $100^{\mathrm{M}, \mathrm{Ant}}$ & 50 & & & Mann et al., 2010a; \\
\hline W297/T298 & $\mathrm{A} / \mathrm{A}$ & & 22 & & $57 \% \mathrm{SB}^{\mathrm{M}, \mathrm{Ag}}$ & Dods and Donnelly, 2015 \\
\hline
\end{tabular}


TABLE 1-Continued

\begin{tabular}{|c|c|c|c|c|c|c|}
\hline Residue & $\begin{array}{l}\text { Mutated } \\
\text { to }\end{array}$ & $\begin{array}{l}\text {-Fold Reduction } \\
\text { Affinity }\end{array}$ & $\begin{array}{l}\text {-Fold Reduction } \\
\text { Potency }\end{array}$ & $\begin{array}{l}\text { Cell Surface } \\
\text { Expression } \\
(\% \text { Wild-Type })\end{array}$ & $\begin{array}{l}\text { Comments and/or Other } \\
\text { Observed Effects }\end{array}$ & Reference \\
\hline R299 ${ }^{\text {ECL2 }}$ & $\mathrm{A}$ & $32^{\mathrm{C}, \mathrm{Ant}}$ & 85 & $\mathrm{WT}^{\mathrm{E}}$ & & Koole et al., 2012a \\
\hline R299 ${ }^{\mathrm{ECL} 2}$ & $\mathrm{~A}$ & $\mathrm{WT}^{\mathrm{M}, \mathrm{Ant}}$ & WT & $40 \%$ Ant & $\Delta \log \tau_{c}=0.60$ & Dods and Donnelly, 2015 \\
\hline $\mathrm{R} 299^{\mathrm{ECL} 2}$ & $\mathrm{~S}$ & $\mathrm{ND}^{\mathrm{C}, \mathrm{Ag}}$ & 43 & $\mathrm{WT}^{\mathrm{cyt}}$ & & Yang et al., 2016 \\
\hline $\mathrm{N} 300^{\mathrm{ECL} 2}$ & $\mathrm{~A}$ & $126^{\mathrm{C}, \text { Ant }}$ & 501 & $\mathrm{WT}^{\mathrm{E}}$ & $\Delta \log \tau_{\mathrm{c}}=0.80$ & Koole et al., 2012a \\
\hline $\mathrm{N} 300^{\mathrm{ECL} 2}$ & $\mathrm{~A}$ & $36^{\mathrm{M}, \mathrm{Ant}}$ & 104 & $18 \%$ Ant & & Dods and Donnelly, 2015 \\
\hline $\mathrm{N} 300^{\mathrm{ECL} 2}$ & $\mathrm{~A}$ & $\mathrm{ND}^{\mathrm{C}, \mathrm{Ag}}$ & ND & $16 \%$ cyt & & Yang et al., 2016 \\
\hline $\mathrm{R} 299 / \mathrm{N}^{\prime} 300^{\dagger}$ & $\mathrm{A} / \mathrm{A}$ & $25^{\mathrm{M}, \text { Ant }}$ & $>3000$ & & & $\begin{array}{l}\text { Mann et al., } 2010 \text { a; } \\
\text { Donnelly, } 2012\end{array}$ \\
\hline R299/N300 & $\mathrm{A} / \mathrm{A}$ & & 331 & & $41 \% \mathrm{SB}^{\mathrm{M}, \mathrm{Ag}}$ & Dods and Donnelly, 2015 \\
\hline $\mathrm{S} 301^{\mathrm{ECL} 2}$ & $\mathrm{~A}$ & $\mathrm{WT}^{\mathrm{C}, \mathrm{Ant}}$ & WT & $63 \%^{\mathrm{E}}$ & & Koole et al., $2012 \mathrm{a}$ \\
\hline N302 $2^{\text {ECL2 }}$ & $\mathrm{A}$ & $25^{\mathrm{C}, \text { Ant }}$ & 16 & $\mathrm{WT}^{\mathrm{E}}$ & $\Delta \log \tau_{c}=0.53$ & Koole et al., $2012 \mathrm{a}$ \\
\hline $\mathrm{S} 301 / \mathrm{N} 302^{\dagger}$ & $\mathrm{A} / \mathrm{A}$ & $\mathrm{WT}^{\mathrm{M}, \mathrm{Ag}}$ & WT & & & Mann et al., 2010a \\
\hline S301/N302 & $\mathrm{A} / \mathrm{A}$ & & WT & & $86 \% \mathrm{SB}^{\mathrm{M}, \mathrm{Ag}}$ & Dods and Donnelly, 2015 \\
\hline N302/M303 & $\mathrm{V} / \mathrm{K}$ & $\mathrm{WT}^{\mathrm{C}, \mathrm{Ag}}$ & 10 & & & Moon et al., 2012 \\
\hline M303 ${ }^{\mathrm{ECL} 2}$ & A & $\mathrm{WT}^{\mathrm{C}, \mathrm{Ant}}$ & WT & $\mathrm{WT}^{\mathrm{E}}$ & & Koole et al., 2012a \\
\hline $\mathrm{N} 304^{\mathrm{ECL} 2}$ & $\mathrm{~A}$ & $\mathrm{WT}^{\mathrm{C}, \mathrm{Ant}}$ & WT & $70 \%^{\mathrm{E}}$ & $\Delta \log \tau_{c}=0.74$ & Koole et al., 2012a \\
\hline $\mathrm{N} 304^{\mathrm{ECL2}}$ & $\mathrm{A}$ & $\mathrm{WT}^{\mathrm{C}, \mathrm{Ag}}$ & WT & $\mathrm{WT}^{\text {cyt }}$ & & Yang et al., 2016 \\
\hline M303/N304 & A/A & $\mathrm{WT}^{\mathrm{M}, \mathrm{Ag}}$ & WT & & & Mann et al., 2010a \\
\hline M303/N304 & $\mathrm{A} / \mathrm{A}$ & & WT & & $91 \% \mathrm{SB}^{\mathrm{M}, \mathrm{Ag}}$ & Dods and Donnelly, 2015 \\
\hline Y305 $5^{5.35 b}$ & $\mathrm{~A}$ & $79^{\mathrm{C}, \mathrm{Ant}}$ & 40 & $52 \%^{\mathrm{E}}$ & & Koole et al., $2012 \mathrm{a}$ \\
\hline $\mathrm{Y}^{3} 05^{5.35 \mathrm{~b}}$ & $\mathrm{~A}$ & $\mathrm{WT}^{\mathrm{M}, \mathrm{Ant}}$ & WT & $22 \%$ Ant & & Dods and Donnelly, 2015 \\
\hline W306 $6^{5.36 b}$ & $\mathrm{~A}$ & $\mathrm{ND}^{\mathrm{C}, \text { Ant }}$ & ND & ND & No receptor expression & Koole et al., $2012 \mathrm{a}$ \\
\hline W306 $6^{5.36}$ & $\mathrm{~A}$ & $109^{\mathrm{M}, \mathrm{Ant}}$ & 206 & $41 \%$ Ant & & Dods and Donnelly, 2015 \\
\hline W306 $6^{5.36}$ & $\mathrm{~A}$ & $\mathrm{ND}^{\mathrm{C}, \mathrm{Ag}}$ & ND & $\mathrm{WT}^{\mathrm{cyt}}$ & & Yang et al., 2016 \\
\hline${ }^{\dagger} \mathrm{Y} 305 / \mathrm{W} 306^{\dagger}$ & $\mathrm{A} / \mathrm{A}$ & $316^{\mathrm{M}, \mathrm{Ant}}$ & 50 & & & $\begin{array}{l}\text { Mann et al., 2010a; } \\
\text { Donnelly, } 2012\end{array}$ \\
\hline Y305/W306 & $\mathrm{A} / \mathrm{A}$ & & 263 & & $60 \% \mathrm{SB}^{\mathrm{M}, \mathrm{Ag}}$ & Dods and Donnelly, 2015 \\
\hline $\mathrm{L} 307^{5.37 \mathrm{~b}}$ & $\mathrm{~A}$ & $13^{\mathrm{C}, \mathrm{Ant}}$ & 25 & $47 \%^{\mathrm{E}}$ & & Koole et al., $2012 \mathrm{a}$ \\
\hline $\mathrm{L} 307^{5.37 b}$ & $\mathrm{~A}$ & & WT & & $87 \% \mathrm{SB}^{\mathrm{M}, \mathrm{Ag}}$ & Dods and Donnelly, 2015 \\
\hline $\mathrm{I} 308^{5.38 b}$ & $\mathrm{~A}$ & & WT & & $78 \% \mathrm{SB}^{\mathrm{M}, \mathrm{Ag}}$ & Dods and Donnelly, 2015 \\
\hline $\mathrm{L}^{3} 07 / \mathrm{I} 308^{\dagger}$ & $\mathrm{A} / \mathrm{A}$ & $251^{\mathrm{M}, \mathrm{Ant}}$ & 6 & & & $\begin{array}{l}\text { Mann et al., 2010a; } \\
\text { Donnelly } 2012\end{array}$ \\
\hline $\mathrm{I} 309^{5.39 b}$ & $\mathrm{~A}$ & $\mathrm{WT}^{\mathrm{M}, \mathrm{Ant}}$ & WT & $\mathrm{WT}^{\mathrm{Ant}}$ & & Dods and Donnelly, 2015 \\
\hline $\mathrm{R} 310^{5.40 \mathrm{~b}}$ & $\mathrm{~A}$ & $10^{\mathrm{M}, \mathrm{Ant}}$ & 1259 & $17 \%$ Ant & & Coopman et al., 2011 \\
\hline $\mathrm{R} 310^{5.40 \mathrm{~b}}$ & $\mathrm{~A}$ & $\mathrm{WT}^{\mathrm{M}, \mathrm{Ant}}$ & 1290 & $19 \%$ Ant & $\Delta \log \tau_{\mathrm{c}}=0.75$ & Dods and Donnelly, 2015 \\
\hline $\mathrm{I} 309 / \mathrm{R} 310^{\dagger}$ & A/A & $50^{\mathrm{M}, \mathrm{Ant}}$ & $>3000$ & & & $\begin{array}{l}\text { Mann et al., 2010a; } \\
\text { Donnelly, } 2012\end{array}$ \\
\hline I309/R310 & $\mathrm{A} / \mathrm{A}$ & & 13,490 & & $13 \% \mathrm{SB}^{\mathrm{M}, \mathrm{Ag}}$ & Dods and Donnelly, 2015 \\
\hline L311/P312 & $\mathrm{A} / \mathrm{A}$ & & WT & & $91 \% \mathrm{SB}^{\mathrm{M}, \mathrm{Ag}}$ & Dods and Donnelly, 2015 \\
\hline A $316^{5.46 b}$ & $\mathrm{~T}$ & $\mathrm{WT}^{\mathrm{C}, \mathrm{Ant}}$ & WT & $<25 \%{ }^{\mathrm{E}}$ & & Koole et al., 2011 \\
\hline $\mathrm{N} 320^{5.50 \mathrm{~b}}$ & $\mathrm{~A}$ & $18^{\mathrm{C}, \text { Ant }}$ & 10 & $\mathrm{WT}^{\mathrm{E} \text {,Ant }}$ & $\Delta \log \tau_{\mathrm{c}}=0.50$ & Wootten et al., 2013c \\
\hline $\mathrm{F} 321^{5.51 \mathrm{~b} \dagger}$ & $\mathrm{A}$ & $\mathrm{WT}^{\mathrm{C}, \mathrm{Ag}}$ & & $\mathrm{WT}^{\mathrm{Ag}}$ & WT cAMP $10^{-7}$ M GLP-1 & Mathi et al., 1997 \\
\hline $\mathrm{L} 322^{5.52 \mathrm{~b} \dagger}$ & $\mathrm{A}$ & $\mathrm{WT}^{\mathrm{C}, \mathrm{Ag}}$ & & $\mathrm{WT}^{\mathrm{Ag}}$ & WT cAMP $10^{-7}$ M GLP-1 & Mathi et al., 1997 \\
\hline $\mathrm{I} 323^{5.53 \mathrm{~b} \dagger}$ & $\mathrm{A}$ & $\mathrm{WT}^{\mathrm{C}, \mathrm{Ag}}$ & & $35 \%$ Ant & WT cAMP $10^{-7}$ M GLP-1 & Mathi et al., 1997 \\
\hline $\mathrm{F} 324^{5.54 \mathrm{~b} \dagger}$ & $\mathrm{A}$ & $\mathrm{WT}^{\mathrm{C}, \mathrm{Ag}}$ & & $\mathrm{WT}^{\mathrm{Ag}}$ & WT cAMP $10^{-7}$ M GLP-1 & Mathi et al., 1997 \\
\hline $\mathrm{I} 325 / \mathrm{F} 326^{\dagger}$ & $\mathrm{A} / \mathrm{A}$ & $\mathrm{WT}^{\mathrm{C}, \mathrm{Ag}}$ & & $\mathrm{WT}^{\mathrm{Ag}}$ & WT cAMP $10^{-7}$ M GLP-1 & Mathi et al., 1997 \\
\hline $\mathrm{V} 327^{5.57 \mathrm{~b}^{\dagger}}$ & $\mathrm{A}$ & $\mathrm{WT}^{\mathrm{C}, \mathrm{Ag}}$ & 15 & $\mathrm{WT}^{\mathrm{Ag}}$ & $42 \%$ cAMP $10^{-7}$ M GLP-1 & Mathi et al., 1997 \\
\hline $\mathrm{I} 328^{5.58 \mathrm{~b} \dagger}$ & $\mathrm{A}$ & $\mathrm{WT}^{\mathrm{C}, \mathrm{Ag}}$ & 9 & $\mathrm{WT}^{\mathrm{Ant}}$ & $39 \%$ cAMP $10^{-7}$ M GLP-1 & Mathi et al., 1997 \\
\hline $\mathrm{C} 329^{5.59 \mathrm{~b} \dagger}$ & $\mathrm{A}$ & $\mathrm{WT}^{\mathrm{C}, \mathrm{Ag}}$ & & $\mathrm{WT}^{\mathrm{Ant}}$ & WT cAMP $10^{-7}$ M GLP-1 & Mathi et al., 1997 \\
\hline $\mathrm{I} 330^{5.60 \mathrm{~b}}$ & $\mathrm{~A}$ & $\mathrm{WT}^{\mathrm{C}, \mathrm{Ag}}$ & & $\mathrm{WT}^{\mathrm{Ag}}$ & WT cAMP $10^{-7}$ M GLP-1 & Mathi et al., 1997 \\
\hline V331 $1^{5.61 b \dagger}$ & $\mathrm{A}$ & $\mathrm{WT}^{\mathrm{C}, \mathrm{Ag}}$ & 14 & $\mathrm{WT}^{\mathrm{Ag}}$ & $45 \%$ cAMP $10^{-7}$ M GLP-1 & Mathi et al., 1997 \\
\hline $\mathrm{I} 332^{5.62 \mathrm{~b}^{\dagger}}$ & $\mathrm{A}$ & $\mathrm{WT}^{\mathrm{C}, \mathrm{Ag}}$ & & $\mathrm{WT}^{\mathrm{Ag}}$ & WT cAMP $10^{-7}$ M GLP-1 & Mathi et al., 1997 \\
\hline $\mathrm{A} 333^{5.63 \mathrm{~b} \dagger}$ & $\mathrm{L}$ & $\mathrm{WT}^{\mathrm{C}, \mathrm{Ag}}$ & & $\mathrm{WT}^{\mathrm{Ag}}$ & WT cAMP $10^{-7}$ M GLP-1 & Mathi et al., 1997 \\
\hline $\mathrm{S} 333^{5.63 \mathrm{~b}}$ & $\mathrm{C}$ & $\mathrm{WT}^{\mathrm{C}, \mathrm{Ant}}$ & WT & $<50 \%{ }^{\mathrm{E}}$ & $\begin{array}{l}\text { For additional residue } \\
\text { substiutions, see Koole } \\
\text { et al., } 2015\end{array}$ & Koole et al., 2011 \\
\hline $\mathrm{K} 334^{5.64 \mathrm{~b} \dagger}$ & A & $\mathrm{WT}^{\mathrm{C}, \mathrm{Ag}}$ & & $\mathrm{WT}^{\mathrm{Ag}}$ & $28 \%$ cAMP $10^{-7}$ M GLP-1 & $\begin{array}{l}\text { Takhar et al., 1996; } \\
\text { Mathi et al., } 1997\end{array}$ \\
\hline $\mathrm{L} 335^{5.65 \mathrm{~b} \dagger}$ & $\mathrm{A}$ & $\mathrm{WT}^{\mathrm{C}, \mathrm{Ag}}$ & & $\mathrm{WT}^{\mathrm{Ag}}$ & WT cAMP $10^{-7}$ M GLP-1 & $\begin{array}{l}\text { Takhar et al., 1996; } \\
\text { Mathi et al., } 1997\end{array}$ \\
\hline $\mathrm{K} 336^{5.66 \mathrm{~b} \dagger}$ & $\mathrm{L}$ & $\mathrm{WT}^{\mathrm{C}, \mathrm{Ag}}$ & & $\mathrm{WT}^{\mathrm{Ag}}$ & WT cAMP $10^{-7}$ M GLP-1 & $\begin{array}{l}\text { Takhar et al., 1996; } \\
\text { Mathi et al., } 1997\end{array}$ \\
\hline K334/K351 & Deletions & $\mathrm{WT}^{\mathrm{C}, \mathrm{Ag}}$ & & $\begin{array}{l}\text { Ag See paper } \\
\text { for details }\end{array}$ & & Takhar et al., 1996 \\
\hline $\mathrm{C} 347^{6.36 \mathrm{~b}}$ & A & & WT & & & Underwood et al., 2013 \\
\hline $\mathrm{R} 348^{6.37 \mathrm{~b} \dagger}$ & $\mathrm{G}$ & $12^{\mathrm{C}, \mathrm{Ag}}$ & ND & $\mathrm{WT}^{\mathrm{mic}}$ & & Heller et al., 1996 \\
\hline $\mathrm{R} 348^{6.37 \mathrm{~b} \dagger}$ & $\mathrm{A}$ & $\mathrm{WT}^{\mathrm{C}, \mathrm{Ag}}$ & & $\mathrm{WT}^{\mathrm{Ag}}$ & WT cAMP $10^{-7}$ M GLP-1 & Takhar et al., 1996 \\
\hline $\mathrm{L} 349^{6.38 \mathrm{~b} \dagger}$ & $\mathrm{A}$ & $\mathrm{WT}^{\mathrm{C}, \mathrm{Ag}}$ & & $76 \% \mathrm{Ag}$ & WT cAMP $10^{-7}$ M GLP-1 & Takhar et al., 1996 \\
\hline $\mathrm{A}-350^{6.39 \mathrm{~b} \dagger}$ & $\mathrm{E}$ & $\mathrm{ND}^{\mathrm{C}, \mathrm{Ag}}$ & & $<10 \%{ }^{\mathrm{Ag}}$ & $5 \%$ cAMP $10^{-7}$ M GLP-1 & Takhar et al., 1996 \\
\hline $\mathrm{A}-350^{6.39 \mathrm{~b} \dagger}$ & $\mathrm{K}$ & $\mathrm{WT}^{\mathrm{C}, \mathrm{Ag}}$ & & $21 \% \mathrm{Ag}$ & $2 \%$ cAMP $10^{-7}$ M GLP-1 & Takhar et al., 1996 \\
\hline
\end{tabular}


TABLE 1-Continued

\begin{tabular}{|c|c|c|c|c|c|c|}
\hline Residue & $\begin{array}{l}\text { Mutated } \\
\text { to }\end{array}$ & $\begin{array}{l}\text {-Fold Reduction } \\
\text { Affinity }\end{array}$ & $\begin{array}{l}\text {-Fold Reduction } \\
\text { Potency }^{*}\end{array}$ & $\begin{array}{c}\text { Cell Surface } \\
\text { Expression } \\
\text { (\% Wild-Type) }\end{array}$ & $\begin{array}{l}\text { Comments and/or Other } \\
\text { Observed Effects }\end{array}$ & Reference \\
\hline $\mathrm{K} 351^{6.40 \mathrm{~b} \dagger}$ & A & $\mathrm{WT}^{\mathrm{C}, \mathrm{Ag}}$ & & $\mathrm{WT}^{\mathrm{Ag}}$ & WT cAMP $10^{-7}$ M GLP-1 & Takhar et al., 1996 \\
\hline T353 $3^{6.42 b}$ & $\mathrm{~A}$ & $\mathrm{ND}^{\mathrm{C}, \mathrm{Ant}}$ & 22 & $30 \%{ }^{\mathrm{E}}, \mathrm{ND}^{\mathrm{Ant}}$ & $\Delta \log \tau_{\mathrm{c}}=0.84$ & Wootten et al., 2013c \\
\hline $\mathrm{H} 363^{6.52 b}$ & $\mathrm{~A}$ & $98^{\mathrm{M}, \mathrm{Ant}}$ & ND & $24 \%$ Ant & & Coopman et al., 2011 \\
\hline H363 ${ }^{6.52 b}$ & $\mathrm{~A}$ & $23^{\mathrm{C}, \mathrm{Ant}}$ & 4 & $59 \%^{\mathrm{E}}, 53 \%$ Ant & $\Delta \log \tau_{c}=1.71$ & Wootten et al., 2013c \\
\hline E364 $6.53 b$ & $\mathrm{~A}$ & $58^{\mathrm{M}, \text { Ant }}$ & 15 & $42 \%$ Ant & & Coopman et al., 2011 \\
\hline $\mathrm{E} 364^{6.53 \mathrm{~b}}$ & $\mathrm{~A}$ & $25^{\mathrm{C}, \mathrm{Ant}}$ & & $51 \%^{\mathrm{E}, \mathrm{Ant}}$ & $\Delta \log \tau_{c}=0.66$ & Wootten et al., 2016 \\
\hline E364 $4^{6.53 b}$ & $\mathrm{~A}$ & $\mathrm{ND}^{\mathrm{C}, \mathrm{Ag}}$ & ND & $6 \%$ cyt & & Yang et al., 2016 \\
\hline E364 $6.53 \mathrm{~b}$ & $\mathrm{Q}$ & $\mathrm{WT}^{\mathrm{C}, \mathrm{Ag}}$ & WT & $42 \%$ cyt & & Yang et al., 2016 \\
\hline $\mathrm{E} 364^{6.53 b}$ & $\mathrm{Q}$ & $0.2^{\mathrm{C}, \mathrm{Ant}}$ & WT & $42 \%{ }^{\text {cyt }}$ & & Yang et al., 2016 \\
\hline E364/E387 & $\mathrm{N} / \mathrm{Q}$ & $\mathrm{ND}^{\mathrm{C}, \mathrm{Ag}}$ & ND & $\mathrm{WT}^{\mathrm{cyt}}$ & & Yang et al., 2016 \\
\hline $\mathrm{E} 364^{6.53 \mathrm{~b}}$ & $\mathrm{Y}$ & $\mathrm{WT}^{\mathrm{C}, \mathrm{Ag}}$ & WT & $\mathrm{WT}^{\mathrm{cyt}}$ & & Yang et al., 2016 \\
\hline $\mathrm{E} 364^{6.53 \mathrm{~b}}$ & $\mathrm{D}$ & $\mathrm{WT}^{\mathrm{C}, \mathrm{Ag}}$ & WT & $53 \%$ cyt & & Yang et al., 2016 \\
\hline F367 $6.56 \mathrm{~b}$ & $\mathrm{~A}$ & $\mathrm{ND}^{\mathrm{C}, \mathrm{Ag}}$ & 72 & $\mathrm{WT}^{\mathrm{cyt}}$ & & Yang et al., 2016 \\
\hline F367 $6.56 b$ & $\mathrm{~A}$ & $32^{\text {C,Ant }}$ & 72 & $\mathrm{WT}^{\mathrm{cyt}}$ & & Yang et al., 2016 \\
\hline F36 $36.56 \mathrm{~b}$ & $\mathrm{I}$ & $\mathrm{ND}^{\mathrm{C}, \mathrm{Ag}}$ & 20 & $\mathrm{WT}^{\mathrm{cyt}}$ & & Yang et al., 2016 \\
\hline F367 $6.56 \mathrm{~b}$ & $\mathrm{H}$ & $7^{\mathrm{C}, \mathrm{Ag}}$ & 131 & $\mathrm{WT}^{\mathrm{cyt}}$ & & Yang et al., 2016 \\
\hline M371 ${ }^{\text {ECL3 }}$ & $\mathrm{A}$ & WT M,Ant & WT & $54 \%$ Ant & & Dods and Donnelly, 2015 \\
\hline D372 $2^{\text {ECL3 }}$ & $\mathrm{A}$ & WT M,Ant & 59 & $13 \%$ Ant & & Dods and Donnelly, 2015 \\
\hline E373 ${ }^{\text {ECL3 }}$ & $\mathrm{A}$ & $\mathrm{WT}^{\mathrm{M}, \mathrm{Ant}}$ & WT & $28 \%$ Ant & & Dods and Donnelly, 2015 \\
\hline H374 ${ }^{\text {ECL3 }}$ & $\mathrm{A}$ & $\mathrm{WT}^{\mathrm{M}, \mathrm{Ant}}$ & WT & $22 \%$ Ant & & Dods and Donnelly, 2015 \\
\hline $\mathrm{H} 374^{\text {ECL3 }}$ & $\mathrm{A}$ & $\mathrm{WT}^{\mathrm{C}, \mathrm{Ag}}$ & WT & $\mathrm{WT}^{\mathrm{cyt}}$ & & Yang et al., 2016 \\
\hline $\mathrm{A}-375^{\mathrm{ECL} 3}$ & $\mathrm{~A}$ & $10^{\mathrm{M}, \mathrm{Ant}}$ & WT & $\mathrm{WT}^{\mathrm{Ant}}$ & & Dods and Donnelly, 2015 \\
\hline $\mathrm{R} 376^{\mathrm{ECL} 3}$ & $\mathrm{G}$ & $\mathrm{WT}^{\mathrm{M}, \mathrm{Ant}}$ & WT & $\mathrm{WT}^{\mathrm{Ant}}$ & & Dods and Donnelly, 2015 \\
\hline R376 ${ }^{\mathrm{ECL} 3}$ & $\mathrm{Q}$ & $\mathrm{WT}^{\mathrm{C}, \mathrm{Ag}}$ & WT & $\mathrm{WT}^{\mathrm{cyt}}$ & & Yang et al., 2016 \\
\hline $\mathrm{G} 377^{\mathrm{ECL} 3}$ & A & $\mathrm{WT}^{\mathrm{M}, \mathrm{Ant}}$ & WT & $19 \%$ Ant & & Dods and Donnelly, 2015 \\
\hline $\mathrm{T} 378^{7.32 \mathrm{~b}}$ & $\mathrm{~A}$ & $\mathrm{WT}^{\mathrm{M}, \mathrm{Ant}}$ & WT & $12 \%$ Ant & & Dods and Donnelly, 2015 \\
\hline L379 $7.33 b$ & $\mathrm{R}$ & $12^{\mathrm{C}, \mathrm{Ag}}$ & 141 & $\mathrm{WT}^{\mathrm{Ag}}$ & & Moon et al., 2015 \\
\hline $\mathrm{L} 379^{7.33 b}$ & $\mathrm{E}$ & $11^{\mathrm{C}, \mathrm{Ag}}$ & 165 & $\mathrm{WT}^{\mathrm{Ag}}$ & & Moon et al., 2015 \\
\hline $\mathrm{L} 379^{7.33 b}$ & $\mathrm{~A}$ & $\mathrm{WT}^{\mathrm{M}, \mathrm{Ant}}$ & WT & $31 \%$ Ant & & Dods and Donnelly, 2015 \\
\hline $\mathrm{R} 380^{7.34 \mathrm{~b}}$ & $\mathrm{D}$ & $21^{\mathrm{C}, \mathrm{Ag}}$ & 1853 & $\mathrm{WT}^{\mathrm{Ag}}$ & & Moon et al., 2015 \\
\hline $\mathrm{R} 380^{7.34 \mathrm{~b}}$ & $\mathrm{G}$ & $4^{\mathrm{C}, \mathrm{Ag}}$ & 40 & $\mathrm{WT}^{\mathrm{Ag}}$ & & Moon et al., 2015 \\
\hline $\mathrm{R} 380^{7.34 \mathrm{~b}}$ & $\mathrm{~A}$ & $128^{\mathrm{M}, \mathrm{Ant}}$ & 263 & $68 \%$ Ant & & Dods and Donnelly, 2015 \\
\hline $\mathrm{R} 380^{7.34 \mathrm{~b}}$ & $\mathrm{Q}$ & $\mathrm{ND}^{\mathrm{C}, \mathrm{Ag}}$ & $\mathrm{ND}$ & $\mathrm{WT}^{\mathrm{cyt}}$ & & Yang et al., 2016 \\
\hline F381 ${ }^{7.35 b}$ & $\mathrm{R}$ & WT $\mathrm{C}, \mathrm{Ag}$ & WT & $\mathrm{WT}^{\mathrm{Ag}}$ & & Moon et al., 2015 \\
\hline F381 & $\mathrm{E}$ & $>200^{\mathrm{C}, \mathrm{Ag}}$ & 234 & $\mathrm{WT}^{\mathrm{Ag}}$ & & Moon et al., 2015 \\
\hline F381 & $\mathrm{A}$ & $\mathrm{WT}^{\mathrm{M}, \mathrm{Ant}}$ & WT & $16 \%$ Ant & & Dods and Donnelly, 2015 \\
\hline F381 & $\mathrm{S}$ & $\mathrm{WT}^{\mathrm{C}, \mathrm{Ag}}$ & WT & $\mathrm{WT}^{\text {cyt }}$ & & Yang et al., 2016 \\
\hline $\mathrm{I} 382^{7.36 \mathrm{~b}}$ & $\mathrm{~A}$ & $\mathrm{WT}^{\mathrm{M}, \mathrm{Ant}}$ & WT & $23 \%$ Ant & & Dods and Donnelly, 2015 \\
\hline $\mathrm{K} 383^{7.37 \mathrm{~b}}$ & $\mathrm{~A}$ & $\mathrm{WT}^{\mathrm{M}, \mathrm{Ant}}$ & 56 & $\mathrm{WT}^{\mathrm{Ant}}$ & $\Delta \log \tau_{c}=1.18$ & Dods and Donnelly, 2015 \\
\hline $\mathrm{L} 384^{7.38 b}$ & $\mathrm{~A}$ & $\mathrm{WT}^{\mathrm{M}, \mathrm{Ant}}$ & WT & $\mathrm{WT}^{\mathrm{Ant}}$ & & Dods and Donnelly, 2015 \\
\hline $\mathrm{L}^{2} 84^{7.38 b}$ & $\mathrm{~A}$ & $\mathrm{ND}^{\mathrm{C}, \mathrm{Ag}}$ & 41 & $\mathrm{WT}^{\mathrm{cyt}}$ & & Yang et al., 2016 \\
\hline $\mathrm{L}^{3} 84^{7.38 b}$ & $\mathrm{~A}$ & $48^{\mathrm{C}, \mathrm{Ant}}$ & 41 & $\mathrm{WT}^{\text {cyt }}$ & & Yang et al., 2016 \\
\hline $\mathrm{L} 384^{7.38 b}$ & V & $\mathrm{ND}^{\mathrm{C}, \mathrm{Ag}}$ & 16 & $\mathrm{WT}^{\text {cyt }}$ & & Yang et al., 2016 \\
\hline F385 & $\mathrm{A}$ & $\mathrm{WT}^{\mathrm{M}, \mathrm{Ant}}$ & WT & $\mathrm{WT}^{\text {Ant }}$ & & Dods and Donnelly, 2015 \\
\hline $\mathrm{T} 386^{7.41 b}$ & $\mathrm{~A}$ & $\mathrm{WT}^{\mathrm{M}, \mathrm{Ant}}$ & WT & $18 \%$ Ant & & Dods and Donnelly, 2015 \\
\hline $\mathrm{E} 387^{7.42 b}$ & $\mathrm{~A}$ & $\mathrm{WT}^{\mathrm{M}, \mathrm{Ant}}$ & WT & $43 \%$ Ant & $\Delta \log \tau_{c}=0.52$ & Dods and Donnelly, 2015 \\
\hline $\mathrm{E} 387^{7.42 b}$ & $\mathrm{~A}$ & $\mathrm{WT}^{\mathrm{M}, \mathrm{Ant}}$ & WT & $\mathrm{WT}^{\mathrm{Ant}}$ & & Coopman et al., 2011 \\
\hline E38 $7.42 b$ & $\mathrm{D}$ & $13^{\mathrm{C}, \mathrm{Ag}}$ & 10 & $\mathrm{WT}^{\mathrm{cyt}}$ & & Yang et al., 2016 \\
\hline $\mathrm{E} 387^{7.42 b}$ & $\mathrm{D}$ & $12^{\mathrm{C}, \mathrm{Ant}}$ & 10 & $\mathrm{WT}^{\text {cyt }}$ & & Yang et al., 2016 \\
\hline $\mathrm{E} 387^{7.42 b}$ & $\mathrm{~N}$ & $\mathrm{WT}^{\mathrm{C}, \mathrm{Ag}}$ & WT & $\mathrm{WT}^{\text {cyt }}$ & & Yang et al., 2016 \\
\hline L388 ${ }^{7.43 b}$ & $\mathrm{~A}$ & $\mathrm{ND}^{\mathrm{C}, \mathrm{Ag}}$ & 208 & $\mathrm{WT}^{\mathrm{cyt}}$ & & Yang et al., 2016 \\
\hline $\mathrm{L}^{2} 88^{7.43 b}$ & $\mathrm{I}$ & $5^{\mathrm{C}, \mathrm{Ag}}$ & 81 & $\mathrm{WT}^{\mathrm{cyt}}$ & & Yang et al., 2016 \\
\hline $\mathrm{L}^{3} 88^{7.43 b}$ & $\mathrm{~F}$ & $\mathrm{WT}^{\mathrm{C}, \mathrm{Ag}}$ & WT & $\mathrm{WT}^{\mathrm{cyt}}$ & & Yang et al., 2016 \\
\hline T391 & A & $\mathrm{WT}^{\mathrm{M}, \mathrm{Ant}}$ & WT & $64 \%^{\mathrm{M}, \mathrm{Ant}}$ & & Coopman et al., 2011 \\
\hline $\mathrm{T} 391^{7.44 \mathrm{~b}}$ & $\mathrm{~A}$ & & WT & $\mathrm{WT}^{\mathrm{Ag}}$ & & Underwood et al., 2011 \\
\hline $\mathrm{S} 392^{7.47 \mathrm{~b}}$ & $\mathrm{~A}$ & $\mathrm{WT}^{\mathrm{C}, \mathrm{Ant}}$ & WT & $\mathrm{WT}^{\mathrm{E}}$, Ant & & Wootten et al., 2013c \\
\hline Q394 & A & $\mathrm{WT}^{\mathrm{C}, \mathrm{Ant}}$ & WT & $\mathrm{WT}^{\mathrm{E}, \text { Ant }}$ & & Wootten et al., 2013c \\
\hline $\mathrm{Q} 394^{7.49 \mathrm{~b}}$ & $\mathrm{~N}$ & $\mathrm{WT}^{\mathrm{C}, \mathrm{Ant}}$ & & $\mathrm{WT}^{\mathrm{E}, \mathrm{Ant}}$ & & Wootten et al., 2016 \\
\hline M397 $7.52 b$ & $\mathrm{~L}$ & $\mathrm{WT}^{\mathrm{C}, \mathrm{Ag}}$ & WT & & & Dong et al., 2012 \\
\hline Y $402^{7.57 b}$ & $\mathrm{~A}$ & $\mathrm{ND}^{\mathrm{C}, \mathrm{Ant}}$ & 10 & $21 \%{ }^{\mathrm{E}}, \mathrm{ND}^{\mathrm{Ant}}$ & $\Delta \log \tau_{c}=1.59$ & Wootten et al., 2013c \\
\hline $\mathrm{C} 403^{7.58 b}$ & $\mathrm{~A}$ & & 5.4 & & & Underwood et al., 2013 \\
\hline $\mathrm{N} 406^{7.61 b}$ & $\mathrm{~A}$ & $\mathrm{WT}^{\mathrm{C}, \mathrm{Ant}}$ & WT & $\mathrm{WT}^{\mathrm{E}, \mathrm{Ant}}$ & & Wootten et al., 2013c \\
\hline $\mathrm{R} 421^{\mathrm{CTT}}$ & $\mathrm{Q}$ & $\mathrm{WT}^{\mathrm{C}, \mathrm{Ant}}$ & WT & $<50 \%{ }^{\mathrm{E}}$ & & Koole et al., 2011 \\
\hline $\mathrm{C} 438^{\mathrm{CTT}}$ & $\mathrm{A}$ & & WT & & & Underwood et al., 2013 \\
\hline $\mathrm{C} 458^{\mathrm{CTT}}$ & $\mathrm{A}$ & & WT & & & Underwood et al., 2013 \\
\hline $\mathrm{C} 462^{\mathrm{CTT}}$ & $\mathrm{A}$ & & WT & & & Underwood et al., 2013 \\
\hline
\end{tabular}

CTT, C-terminal tail; SB, specific binding of radiolabeled ligand; WB, Western blotting.

${ }^{\dagger}$ Note that potency changes can be due to changes in affinity, efficacy, and/or cell surface expression, and hence should be interpreted with caution, especially when either the affinity and/or expression levels are not known.

*Also included in double mutations. 


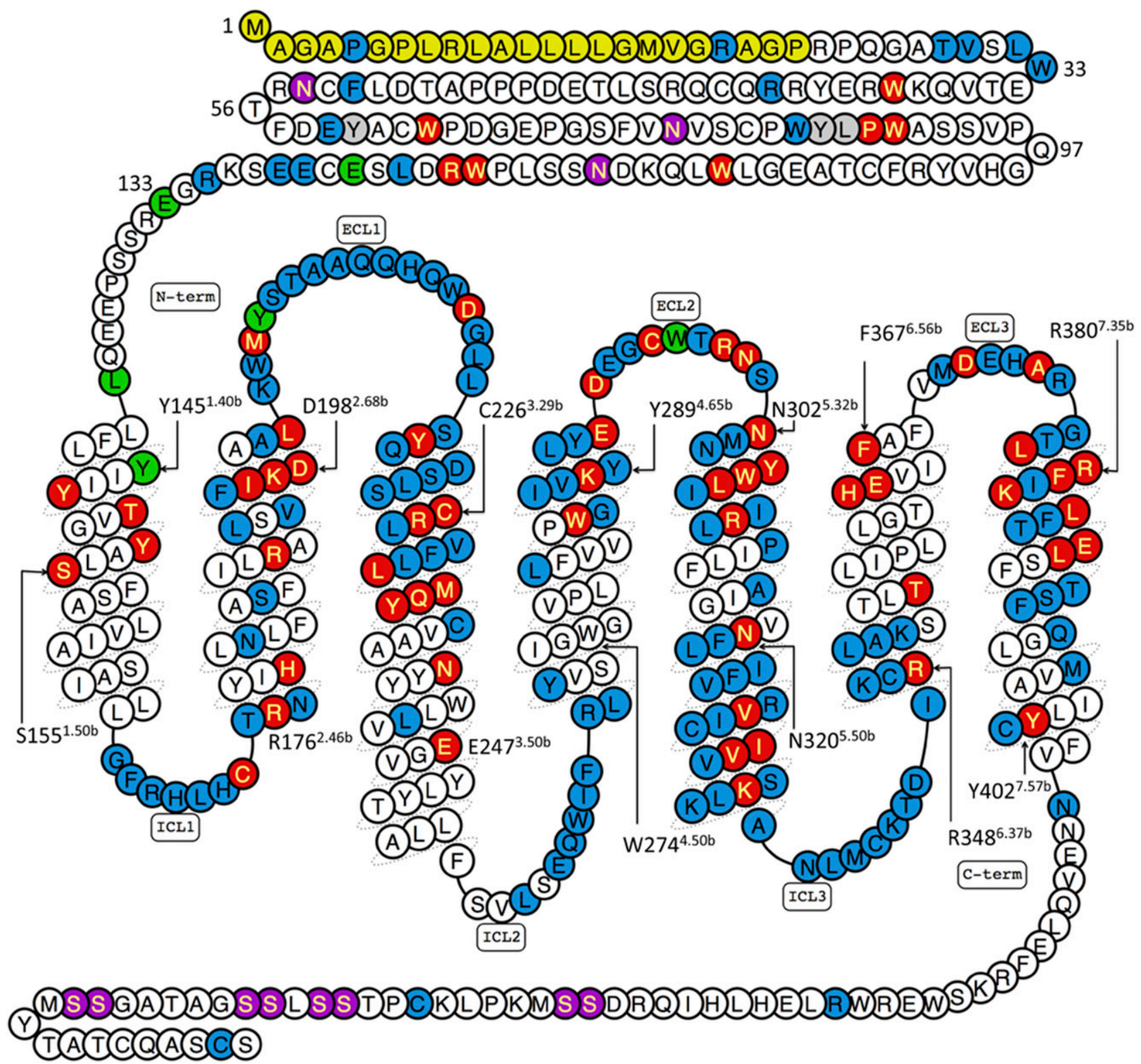

Fig. 3. Summary of GLP-1R mutagenesis. A snake plot of GLP-1R from http://www.gpcrdb.org that has been colored (see Key) to highlight the location of signal peptide, glycosylation, and phosphorylation sites, as well as the mutated residues in Table 1 . It should be noted that the color coding of 74 of the 195 mutated residues (W39, W72, W87, W91, W110, F169-C174, R176, N177, H180, N182, A200-Q213, W214-G225, R227-F230, L232, M233, E262L268, F321-I332, K334-K336, and R348-K351) reflects the effects of rat GLP-1R mutations projected on the hGLP-1R amino acid sequence. The color coding of 27 of the 185 residues (K202, W203, S206-Q211, Q213, W214, G216-Q221, S223-G225, R227-F230, L332, M233, I325, and F326) reflects the effect of double mutations, not single-point mutations. Information on the fold change in ligand affinity and potency as well as expression levels of the GLP-1R mutants is reported in Table 1.

solved that contained 11 thermo-stabilizing mutations and a T4 lysozyme fusion partner inserted into ICL2 (PDB: 5EE7) (Jazayeri et al., 2016). In this structure, a small-molecule antagonist (MK-0893) is bound to an extrahelical allosteric binding site that is distinct from the orthosteric peptide ligand binding site, and the receptor adopts a similar overall conformation as observed in the previously solved GCGR crystal structure.
Analogs of MK-0893 bind GLP-1R with moderate micromolar affinity (Xiong et al., 2012), suggesting that the GCGR crystal structures may also offer a template for the design of small-molecule ligands that target the same extrahelical allosteric binding site in GLP-1R. Even though the general fold of the TM helices is similar to those observed in classes A and C GPCR crystal structures, both $\mathrm{CRF}_{1} \mathrm{R}$ and GCGR structures assume more 
open conformations toward the extracellular side than any other GPCR of known structure. In addition to this open 7TMD pocket, the GCGR structure revealed two other distinct structural features that are not observed in the $\mathrm{CRF}_{1} \mathrm{R}$ structure, namely, a long $\mathrm{N}$-terminal extension of TM helix 1 (defined as stalk region) and a long helix 8 at the $\mathrm{C}$-terminal end of the receptor that is differently oriented than helix 8 observed in the crystal structures of other GPCRs (Hollenstein et al., 2014). Although the distinct orientation of helix 8 in the GCGR structure may be a result of crystal lattice contacts, the stalk is proposed to play a role in the positioning of the ECD relative to the 7TMD (Siu et al., 2013). The electron microscopy map of the full-length GCGR stabilized by a monoclonal antibody (mAb23) suggests that mAb23 interacts with the ECD (preventing glucagon from binding to the receptor) and stabilizes GCGR in an open conformation in which the TM helix 1 stalk connects the ECD and 7TMD and the ECD is almost perpendicular to the membrane surface (Yang et al., 2015b). Hydrogendeuterium exchange studies have indicated that des$\mathrm{His}^{1}-\left[\mathrm{Nle}^{9}-\mathrm{Ala}^{11}-\mathrm{Ala}^{16}\right]$-glucagon- $\mathrm{NH}_{2}$ peptide antagonist binding protects the $\alpha$-helical conformation of the stalk region of GCGR, consistent with molecular dynamicssimulation studies (Yang et al., 2015b). Full-length crystal structure of GLP-1R and other class B GPCRs are required to show whether the stalk region observed in the GCGR 7TMD crystal structure is indeed a conserved structural feature among secretin-like receptors. The GCGR structure lacks the presence of a peptide or small-molecule ligand, but there is clearly a deep and extensive binding pocket comprised of the TM helices at the extracellular side of the 7TM bundle. A glucagonbound full-length GCGR model (Siu et al., 2013) and models of agonist-docked full-length GLP-1R (Dods and Donnelly, 2015; Wootten et al., 2016; Yang et al., 2016) have been proposed based on information from a variety of sources, including the crystal structures of the GCGR 7TMD (Siu et al., 2013), the antibody-bound GCGR ECD (Koth et al., 2012), the GLP-1-bound GLP-1R ECD (Underwood et al., 2010), and molecular dynamics simulations (Wootten et al., 2016; Yang et al., 2016). Although such models can be largely consistent with the results of mutation studies of GLP-1R (Table 1) (Dods and Donnelly, 2015; Wootten et al., 2016; Yang et al., 2016), and can provide insights into GLP-1R-specific mechanisms of ligand-binding selectivity (Yang et al., 2016) and biased signaling (Wootten et al., 2016), a complete interpretation of the mutagenesis data requires a high-resolution structure of the ligand-bound GLP-1R, and, hence, progress in this area is required and keenly anticipated.

b. Mutagenesis of the 7TMD. Although there are numerous molecular pharmacological analyses of various ligands (both peptidic and nonpeptidic) acting at GLP-1R, this section of the review will focus only on those studies that highlight the action of GLP-1 itself.
Likewise, whereas GLP-1R can signal through several pathways, this section will focus on the effects of sitedirected mutagenesis on signaling through the Gs protein that raises intracellular cAMP levels. The ability of GLP-1R to activate other G proteins and pathways will be examined in more detail below. The mutagenesis data are summarized in Table 1 and Fig. 3, with the TM numbering following the convention described by Wootten et al. (2013c).

i. TM1 $\left(S 129^{1.24 b}-L 167^{1.62 b}\right)$. The first TM helix of GLP-1R (TM1) contains two single-nucleotide polymorphism (SNP) sites at positions $131^{1.26 \mathrm{~b}}(\mathrm{Arg} / \mathrm{Asn})$ and $149^{1.44 \mathrm{~b}}$ (Thr/Met). Although the R131 ${ }^{1.26 \mathrm{~b}} \mathrm{~N}$ mutation did not significantly affect GLP-1 action (Koole et al., 2011), Beinborn and colleagues showed that the $\mathrm{T} 149^{1.44 \mathrm{~b}} \mathrm{M}$ mutation (which has been associated with T2DM) led to a 60-fold reduction in GLP-1 affinity and a 30-fold reduction in potency (Tokuyama et al., 2004; Beinborn et al., 2005). The importance of this residue for GLP-1 action has been confirmed subsequently, demonstrating that the same mutation resulted in a 250-fold reduced affinity and a 160 -fold reduced potency, whereas substitution by a variety of other residue types also led to compromised affinity and signaling (Koole et al., 2015), with the exception of the $\mathrm{T} 149^{1.44 b} \mathrm{~S}$ mutant, which has similar ligand affinity and potency as wild-type GLP-1R (Yang et al., 2016). Mutation of Y148 ${ }^{1.43 b}$ to Ala, Asn, or Phe diminished GLP-1 affinity and potency (Yang et al., 2016), while that of Y152 $2^{1.47 b}$ to Ala reduced GLP-1 affinity by 30-fold-potency was compromised as well, although in this case the $B_{\max }$ was low (Coopman et al., 2011), whereas $\mathrm{Y} 152^{1.47 \mathrm{~b}} \mathrm{H}$ does not affect either ligand affinity or potency (Yang et al., 2016). Mutation of the equivalent residue (Y149 $9^{1.47 b}$ ) in GCGR also caused low expression levels, whereas the mutation to His was expressed well and displayed sixfold reduced affinity. Mutation of S155 $5^{1.50 \mathrm{~b}}$ to Ala, in contrast, reduced coupling [efficacy $\left(\mathrm{E}_{\mathrm{max}}\right)$, i.e., the maximal response $=38 \%, \Delta \log \tau_{\mathrm{c}}=$ 0.75] with a minor effect on affinity (Wootten et al., 2013c).

ii. ICL1 (G168-C174). The first residue in the loop is the site of a known SNP (Gly to Ser), but the simulated mutation had no effect on GLP-1 action (Koole et al., 2011). Residues comprising the region between F169 ${ }^{\text {ICL1 }}$ and $\mathrm{C} 174^{\mathrm{ICL} 1}$ in the first intracellular loop were systematically mutated to Ala, but no significant effect upon GLP-1 action was found (Mathi et al., 1997). However, C174 ${ }^{\text {ICL1 }}$ has also been mutated and exhibited a sixfold reduced potency when mutated to Ala and a sevenfold reduced potency when mutated to Ser (Underwood et al., 2013).

iii. TM2 (T175 $\left.2.45 b-L 201^{2.71 b}\right)$. A total of 17 sites within TM2 have been subjected to site-directed mutagenesis (Mathi et al., 1997; Xiao et al., 2000; López de Maturana and Donnelly, 2002; Underwood et al., 2011; Coopman et al., 2011; Moon et al., 2012; Wootten et al., 2013b; Yang et al., 2016). Of these, eight mutations displayed GLP-1 pharmacology similar to the wild-type receptor $\left(\mathrm{T} 175^{2.45 \mathrm{~b}}, \mathrm{~N} 177^{2.47 \mathrm{~b}}, \mathrm{~N} 182^{2} \cdot 52 \mathrm{~b}\right.$, and $\mathrm{A} 200^{2.70 \mathrm{~b}}$ in rat GLP-1R, and S186 $6^{2.56 \mathrm{~b}}, \mathrm{~L} 192^{2.62 \mathrm{~b}}, \mathrm{~V} 194^{2.64 \mathrm{~b}}$, and 
F195 ${ }^{2.65 b}$ in hGLP-1R). In contrast, R176 ${ }^{2.46 b}$ displayed compromised signaling with approximately 13-fold right-shifted potency and $\mathrm{E}_{\max }$ reduced to $26 \%$ (Mathi et al., 1997). The mutation of the conserved $\mathrm{H} 180^{2.50 \mathrm{~b}}$ to Arg resulted in a 21-fold reduced affinity (Heller et al., 1996), whereas substitution to Ala had led to no detectable binding, a 12-fold reduction in potency, and an $\mathrm{E}_{\max }$ of $30 \%\left(\Delta \log \tau_{c}=0.86\right)$ (Wootten et al., 2013c). R190 $0^{2.60}$ has been mutated in multiple different studies: Xiao et al. (2000) found that its mutation to Ala in rat GLP$1 \mathrm{R}$ resulted in $>20$-fold reduced affinity and only $27 \%$ of the wild-type response to $100 \mathrm{nM}$ GLP-1; Coopman et al. (2011) observed a more than 30 -fold reduction in affinity and 270 -fold reduction in potency; Wootten et al. (2013c) described a 20 -fold reduction in GLP-1 affinity with a 34 -fold reduction in potency and $56 \% \mathrm{E}_{\max }\left(\Delta \log \tau_{\mathrm{c}}=\right.$ 0.53); and Yang et al. (2016) reported a diminished GLP-1 affinity and potency for the $\mathrm{R} 190^{2.60 \mathrm{~b}} \mathrm{~A}$ mutant, and a 32-fold reduction in GLP-1 affinity with a 17-fold reduction in potency for the $\mathrm{R} 190^{2.60 \mathrm{~b}} \mathrm{~K}$ mutant. The latter GCGR-mimicking $\mathrm{R} 190^{2.60 \mathrm{~b}} \mathrm{~K}$ mutant does not affect binding affinity of the glucagon mimetic GLP-1 $\mathrm{Glu}^{9} \mathrm{Gln}$ point mutant, suggesting that this residue plays a role in GLP-1 versus glucagon selectivity (Yang et al., 2016). Moon et al. (2012) mutated I196 $6^{2.66 \mathrm{~b}}$ to Ser and observed normal affinity but no activity. K197 $2.67 \mathrm{~b}$ has been mutated to Ala by both Xiao et al. (2000) and Coopman et al. (2011); the former (rat GLP-1R) showed a fivefold reduction in affinity but only $25 \%$ of the wildtype response to $100 \mathrm{nM}$ GLP-1, whereas the latter (hGLP-1R) observed a 28 -fold reduction in affinity and a 630-fold reduction in potency. Yang et al. (2016) showed that mutation of $\mathrm{K} 197^{2.67 \mathrm{~b}}$ to Gln and Ile also diminished GLP-1 affinity and potency in hGLP-1R, whereas the $\mathrm{K} 197^{2.67 \mathrm{~b}} \mathrm{R}$ mutant reduced GLP-1 potency by 23 -fold. The equivalent residues have been mutated in the closely related receptors GCGR (I194 $\left.{ }^{2.67 b}\right)$ and GIPR (R190 $\left.{ }^{2.67 b}\right)$, respectively, with significant impact on their pharmacology and, in the case of GCGR, an association with $\mathrm{Asp}^{3}$ of the ligand (Perret et al., 2002; Runge et al., 2003a; Yaqub et al., 2010; Siu et al., 2013). The neighboring residue D198 ${ }^{2.68 \mathrm{~b}}$ has also been mutated by several groups: Xiao et al. (2000) observed a 10 -fold reduction in affinity and only $20 \%$ of the wild-type response to $100 \mathrm{nM}$ GLP-1 at rat GLP-1R $\left(\mathrm{D} 198^{2.68 \mathrm{~b}} \mathrm{~A}\right)$; López de Maturana and Donnelly (2002) demonstrated a more than 60 -fold reduction in affinity and a more than 40-fold reduction in potency for GLP-1 at rat GLP-1R $\left(\mathrm{D} 198^{2.68 \mathrm{~b}} \mathrm{~A}\right)$; Coopman et al. (2011) described a more than 40 -fold reduction in affinity but a more substantial 980-fold reduction in potency at hGLP-1R (D198 $\left.{ }^{2.68 \mathrm{~b}} \mathrm{~A}\right)$; Yang et al. (2016) showed that in addition to $\mathrm{D} 198^{2.68 \mathrm{~b}} \mathrm{~A}$, $\mathrm{D} 198^{2.68 \mathrm{~b}} \mathrm{~N}$ and $\mathrm{D} 198^{2.68 \mathrm{~b}} \mathrm{E}$ mutants also diminished GLP-1 and exendin-4 affinity and GLP-1 potency.

iv. ECL1 (K202-L218). The ECL1 of rat GLP-1R was scanned using double-Ala mutagenesis by López de Maturana et al. (2004), and only the double mutation of
M204 ${ }^{\text {ECL1 }}$-Y205 ${ }^{\text {ECL1 }}$ displayed some change from the wild-type (87-fold decrease in potency and 37-fold decrease in affinity). M204 ${ }^{\mathrm{ECL} 1} \mathrm{~A}$ and $\mathrm{Y} 205^{\mathrm{ECL} 1} \mathrm{~A}$ single mutants exhibited wild-type GLP-1 pharmacology in rat GLP-1R (López de Maturana et al., 2004), but diminished GLP-1 affinity and potency in hGLP-1R in another study (Yang et al., 2016). Although the W214 ${ }^{\mathrm{ECL} 1}$ $\mathrm{D} 215^{\mathrm{ECL} 1}$ double mutation to Ala showed wild-type properties, Xiao et al. (2000) have reported that the single D215 ${ }^{\text {ECL1 }}$-A mutation has a fourfold reduced affinity and only $57 \%$ of the wild-type cAMP production when treated with $100 \mathrm{nM}$ GLP-1. Yang et al. (2016) showed that $\mathrm{Q} 211^{\mathrm{ECL} 1} \mathrm{D}, \mathrm{Q} 211^{\mathrm{ECL} 1} \mathrm{R}, \mathrm{H} 212^{\mathrm{ECL} 1} \mathrm{~A}$, $\mathrm{W} 214^{\mathrm{ECL} 1} \mathrm{~V}$, and $\mathrm{L} 217^{\mathrm{ECL} 1} \mathrm{~A}$ single amino acid mutants did not affect GLP-1R pharmacology, whereas $W 203^{\mathrm{ECL} 1} \mathrm{~A}$ and $\mathrm{Y} 220^{\mathrm{ECL} 1} \mathrm{D}$ diminished GLP-1 affinity and potency. In contrast, the equivalent region in GCGR plays a critical role in peptide ligand recognition with 15 residues between L198 ${ }^{\mathrm{ECL} 1}$ and A220 ${ }^{\mathrm{ECL} 1}$ affecting peptide response (Roberts et al., 2011; Siu et al., 2013).

v. TM3 $\left(S 219^{3.22 b}-A 256^{3.59 b}\right)$. Although the substitution of $\mathrm{C} 236^{3.39 \mathrm{~b}}$ appeared to have minimal effects on GLP-1 pharmacology, the mutation of the conserved $\mathrm{C} 226^{3.29 \mathrm{~b}}$ in two studies led to either no detectable activity or a 25 -fold reduction in GLP-1 affinity with 38-fold lower potency; the latter was thought to be the result of the breaking of a disulfide bond and the creation of a hydrophobic side chain at position 296 (Mann et al., 2010a; Underwood et al., 2013). The equivalent cysteine in GCGR $\left(\mathrm{C} 224^{3.39 b}\right)$ was also shown to be important for glucagon potency (Prevost et al., 2010; Roberts et al., 2011). The remaining residues spanning $\mathrm{S} 219^{3.22 \mathrm{~b}}$ to $\mathrm{F} 230^{3.33 \mathrm{~b}}$ were included in the double-alanine screening by López de Maturana et al. (2004), and all were found to display wild-typelike GLP-1 pharmacology, although Xiao et al. (2000) reported that the single $\mathrm{R} 227^{3.30 \mathrm{~b}} \mathrm{~A}$ mutation exhibited a more than 20-fold reduced GLP-1 affinity; the equivalent mutation in GCGR $\left(\mathrm{R} 225^{3.30 \mathrm{~b}} \mathrm{~A}\right)$ had no detectable peptide binding (Siu et al., 2013). The double substitution of L232 $2^{3.35 \mathrm{~b}} / \mathrm{M} 233^{3.36 \mathrm{~b}}$ with V/T reduced affinity by 10-fold and potency by 100-fold (Moon et al., 2012), whereas the single substitution of $\mathrm{M} 233^{3.36 \mathrm{~b}}$ with A and T abolished GLP-1 affinity and potency (Yang et al., 2016). The single-alanine substitutions at $Q 234^{3.37 b}$ and Y235 ${ }^{3.38 b}$, respectively, caused a 13 - and 24 -fold reduced affinity with 45 - and 23 -fold reduced potency (Coopman et al., 2011), whereas the $\mathrm{M} 233^{3.36 \mathrm{~b}} \mathrm{~F}$ and $\mathrm{Q} 234^{3.37 \mathrm{~b}} \mathrm{~N}$ mutants did not affect GLP-1 affinity or potency (Yang et al., 2016). The $\mathrm{Q} 234^{3.37 b} \mathrm{E}$ substitution diminished GLP-1 affinity, which was restored by the mutation of $\mathrm{Glu}^{9}$ of GLP-1 into the corresponding Gln residue in glucagon (Yang et al., 2016). Position 3.37b has also been implicated in playing a role in the ligand-binding affinity of GCGR and GIPR (Yaqub et al., 2010; Siu et al., 2013). Replacement of $N 240^{3.43 b}$ with Ala in rat GLP-1R reduced affinity by more than 20 -fold and 
severely impeded cAMP production induced by $100 \mathrm{nM}$ GLP-1 (Xiao et al., 2000), whereas the equivalent replacement in hGLP-1R had minimal effects on affinity but reduced $\mathrm{E}_{\max }$ of GLP-1 to $78 \%\left(\Delta \log \tau_{\mathrm{c}}=0.67\right.$; Wootten et al., 2013c). Likewise, in GCGR the $\mathrm{N} 238^{3.43 b}$ A mutation had no effect on affinity, whereas in GIPR the $\mathrm{N} 230^{3.43 \mathrm{~b}} \mathrm{~A}$ mutant reduced potency by sevenfold. In contrast, substitution of the conserved $\mathrm{E} 247^{3.50 \mathrm{~b}}$ to Ala in hGLP-1R reduced potency by 14-fold and $\mathrm{E}_{\max }$ to $19 \%\left(\Delta \log \tau_{\mathrm{c}}=0.99\right.$; Wootten et al., 2013c), but the equivalent mutation in rat GLP-1R had no major effect (Xiao et al., 2000).

vi. ICL2 (F257-I265). Mutations in the second intracellular loop to date have not shown any effects on GLP-1 pharmacology with the SNP swap Phe/Leu at 260 (Koole et al., 2011) and an Ala scan of E262 ${ }^{\mathrm{ICL} 2}$. I265 ${ }^{\text {ICL2 }}$ (Mathi et al., 1997) displaying wild-type-like properties.

vii. TM4 (F266 $\left.6^{4.42 b}-Y 291^{4.62 b}\right)$. Two sites in TM4 have been highlighted as being key to GLP-1 activity. The first is W284 ${ }^{4.60 \mathrm{~b}}$, which, when mutated to Ala, led to a 32 -fold reduced affinity and a 1350 -fold reduced potency (Coopman et al., 2011). The second important site in this helix is $\mathrm{K} 288^{4.64 \mathrm{~b}}$, which, when mutated to Ala or Leu in rat GLP-1R, respectively, resulted in a 79and 63-fold reduction in affinity with a 251- and 79-fold lower potency (Al-Sabah and Donnelly, 2003b). The $\mathrm{K} 288^{4.64 \mathrm{~b}} \mathrm{~L}$ mutant also abolished GLP-1 affinity and potency in hGLP-1R (Yang et al., 2016). However, the $\mathrm{K} 288^{4.64 \mathrm{~b}}$ to Ala mutation in hGLP-1R caused a 126 -fold reduction in affinity with no detectable cAMP production (Koole et al., 2012a) and substitution of K286 $6^{4.64 b}$ in GCGR abolished radiolabeled ligand binding (Siu et al., 2013). The following mutations did not affect GLP-1 pharmacology: F266 $6^{4.42 \mathrm{~b}} \mathrm{~A}, \mathrm{R} 267^{4.43 \mathrm{~b}} \mathrm{~A}, \mathrm{~L} 268^{4.44 \mathrm{~b}} \mathrm{~A}$, $\mathrm{L} 278^{4.54 \mathrm{~b}} \mathrm{M}, \quad \mathrm{G} 285^{4.61 \mathrm{~b}} \mathrm{~A}, \quad \mathrm{I} 286^{4.62 \mathrm{~b}} \mathrm{~A} / \mathrm{V} 287^{4.63 \mathrm{~b}} \mathrm{~A}$, $\mathrm{I} 286^{4.62 \mathrm{~b}} \mathrm{~A}, \mathrm{~V} 287^{4.63 \mathrm{~b}} \mathrm{~A}, \mathrm{Y} 289^{4.65 \mathrm{~b}} \mathrm{~A}, \mathrm{~L} 290^{4.66 \mathrm{~b}} \mathrm{~A} /$ $\mathrm{Y} 291^{4.67 \mathrm{~b}} \mathrm{~A}, \mathrm{~L} 290^{4.66 \mathrm{~b}} \mathrm{~A}$, and $\mathrm{Y} 291^{4.67 \mathrm{~b}} \mathrm{~A}$ (Mathi et al., 1997; Mann et al., 2010a; Underwood et al., 2010; Koole et al., 2012a).

viii. ECL2 (E292-Y305). The second extracellular loop is an important region for peptide ligand recognition (Mann et al., 2010a; Donnelly, 2012; Koole et al., 2012a; Moon et al., 2012; Dods and Donnelly, 2015). Aside from the key disulfide-forming C296 ${ }^{\mathrm{ECL} 2}$, which has been shown to be crucial for affinity in rat GLP-1R and both affinity and coupling in hGLP-1R (Mann et al., 2010a; Koole et al., 2012a; Underwood et al., 2011), residues E292 ${ }^{\mathrm{ECL} 2}, \mathrm{D} 293^{\mathrm{ECL} 2}$ E294 ${ }^{\mathrm{ECL} 2}$, W297 ${ }^{\mathrm{ECL} 2}$, $\mathrm{R}^{2} 99^{\mathrm{ECL} 2}, \mathrm{~N} 300^{\mathrm{ECL} 2}, \mathrm{~N} 302^{\mathrm{ECL} 2}$, and $\mathrm{N} 304^{\mathrm{ECL} 2}$ all play a role in GLP-1 action (Y305 $5^{5.35 b}$ is involved too-see TM5 below). Koole et al. (2012a) demonstrated that the individual substitutions E292 ${ }^{\mathrm{ECL} 2}$ and D293 ${ }^{\mathrm{ECL} 2}$ in hGLP-1R caused, respectively, $\geq 100$-fold reduced affinity and potency $\left(\Delta \log \tau_{\mathrm{c}}=0.57\right)$ and a 25 -fold reduced affinity with 16 -fold reduced potency, but no change in efficacy. Double mutations of both sites in rat GLP-1R and hGLP-1R support the importance of these residues in GLP-1 pharmacology (Mann et al., 2010a; Dods and Donnelly, 2015). Substitution by Ala of the equivalent E290 ${ }^{\text {ECL2 }}$ and N291 ${ }^{\text {ECL2 }}$ in GCGR led to, respectively, no detectable radioligand binding and a threefold reduction in affinity (Siu et al., 2013). The region encompassing W297 ${ }^{\mathrm{ECL} 2}-\mathrm{N} 300^{\mathrm{ECL} 2}$ was identified as essential in rat GLP-1R and hGLP-1R by double-Ala scanning, whereas the residues spanning S301 ${ }^{\mathrm{ECL} 2}-\mathrm{N} 304^{\mathrm{ECL} 2}$ did not alter GLP-1 pharmacology (Mann et al., 2010a; Donnelly, 2012; Dods and Donnelly, 2015). However, individual substitutions in hGLP-1R by Koole et al. (2012a) demonstrated that, along with E294 ${ }^{\mathrm{ECL} 2}$, $\mathrm{W} 297^{\mathrm{ECL} 2}$, and R299 ${ }^{\mathrm{ECL} 2}$, amino acids $\mathrm{N} 300^{\mathrm{ECL} 2}$, $\mathrm{N} 302^{\mathrm{ECL} 2}$, and N304 ${ }^{\mathrm{ECL} 2}$ were also important (Table 1). In GCGR, the equivalent tryptophan (W295 ${ }^{\mathrm{ECL} 2}$ ) is indispensible for affinity (Siu et al., 2013). The double mutation of N302 $2^{\text {ECL2 }}-\mathrm{M} 303^{\text {ECL2 }}$ to Val-Lys caused a small affinity reduction with a 10-fold decrease in potency (Moon et al., 2012). Residues that appear to be unimportant for GLP-1-mediated production of cAMP are $\mathrm{G} 295^{\mathrm{ECL} 2}, \mathrm{~T} 298^{\mathrm{ECL} 2}, \mathrm{~S} 301^{\mathrm{ECL} 2}$, and $\mathrm{M} 303^{\mathrm{ECL} 2}$ (Mann et al., 2010a; Koole et al., 2012a; Dods and Donnelly, 2015), which correlates with available data for GCGR (Siu et al., 2013).

ix. TM5 (W306 $\left.6^{5.36 b}-K 336^{5.66 b}\right)$. Three double-Ala scan mutations have identified the extracellular end of TM5 of rat GLP-1R as being important for GLP-1 recognition, with substitutions of $\mathrm{Y} 305^{5.35 \mathrm{~b}} / \mathrm{W} 306^{5.36 \mathrm{~b}}$ (this is the ECL2-TM5 interface), L30 $7^{5.37 b} / 1308^{5.38 b}$, and $1309^{5.39 b} / \mathrm{R} 310^{5.40 \mathrm{~b}}$ with Ala residues displaying significantly reduced affinity and potency (Mann et al., 2010a). The equivalent double substitutions in hGLP$1 \mathrm{R}$ supported a role for Y $305^{5.35 \mathrm{~b}} / \mathrm{W} 306^{5.36 \mathrm{~b}}$ and I309 $9^{5.39 \mathrm{~b}} / \mathrm{R} 310^{5.40 \mathrm{~b}}$. However, whereas single mutations of Y305 $5^{5.35 \mathrm{~b}}$, L30 $7^{5.37 \mathrm{~b}}$, and I308 $8^{5.38 \mathrm{~b}}$ to Ala did not significantly alter GLP-1 pharmacology in one study (Dods and Donnelly, 2015), the individual substitutions of Y305 $5.35 \mathrm{~b}$ and L307 $7^{5.37 \mathrm{~b}}$ with Ala resulted in 79-fold decreased affinity with 40-fold reduced potency and 13-fold reduced affinity with 25 -fold lowered potency $\left(\Delta \log \tau_{c}=0.49\right.$ ), respectively, in a second (Koole et al., 2012a). Although W306 $6^{5.36 \mathrm{~b}}$ was not expressed in levels sufficient for analysis in Chinese hamster ovary (CHO) cells (Koole et al., 2012a), in HEK293 cells its mutation to Ala resulted in $>100$-fold reduced affinity and $>200$ fold reduced potency (Dods and Donnelly, 2015). $\mathrm{R} 310^{5.40 \mathrm{~b}} \mathrm{~A}$ resulted in a $>1200$-fold reduced potency, with little effect upon affinity, implying that it plays a key role in agonist-induced signaling (Coopman et al., 2011; Dods and Donnelly, 2015) $\left(\Delta \log \tau_{c}=0.75\right)$. The equivalent mutation in GIPR, R300 ${ }^{5.40 \mathrm{~b}} \mathrm{~A}$, caused a 42-fold reduced affinity and $86 \% \mathrm{E}_{\max }$ (Yaqub et al., 2010). This first part of TM5 is also involved in glucagon affinity in GCGR, with several mutations showing some effect on binding (Siu et al., 2013). N320 ${ }^{5.50 \mathrm{~b}} \mathrm{~A}$ had a modest effect on GLP-1 action with an 18-fold reduced 
affinity and a 10 -fold decreased potency $\left(\Delta \log \tau_{c}=0.50\right)$ (Wootten et al., 2013c). Takhar et al. (1996) and Mathi et al. (1997) Ala scanned the C-terminal end of TM5, demonstrating that V $327^{5.57 \mathrm{~b}}$, I328 ${ }^{5.58 \mathrm{~b}}$, V331 ${ }^{5.61 \mathrm{~b}}$, and K334 $4^{5.64}$ had reduced GLP-1 affinity and activity. However, the mutations of $\mathrm{A} 316^{5.46} \mathrm{~T}, \mathrm{~F} 321^{5.51} \mathrm{~A}, \mathrm{~L} 322^{5.52 \mathrm{~b}} \mathrm{~A}$, $\mathrm{I} 323^{5.53 \mathrm{~b}} \mathrm{~A}, \mathrm{~F} 324^{5.54 \mathrm{~b}} \mathrm{~A}, \mathrm{~V} 325^{5.55 \mathrm{~b}} \mathrm{~A}, \mathrm{R} 326^{5.56 \mathrm{~b}} \mathrm{~A}, \mathrm{C} 329^{5.59 \mathrm{~b}} \mathrm{~A}$, $\mathrm{I} 330^{5.60 \mathrm{~b}} \mathrm{~A}, \mathrm{~V} 332^{5.62 \mathrm{~b}} \mathrm{~A}, \mathrm{~S} 333^{5.63 \mathrm{~b}} \mathrm{~A}, \mathrm{~S} 333^{5.63 \mathrm{~b}} \mathrm{C}, \mathrm{L} 335^{5.65 \mathrm{~b}} \mathrm{~A}$, and $\mathrm{K} 336^{5.66 \mathrm{~b}} \mathrm{~A}$ did not greatly alter GLP-1 pharmacology (Mathi et al., 1997; Koole et al., 2011, 2012a).

x. ICL3 (A337-T343). ICL3 does not appear to play a critical role in GLP-1R pharmacology because Takhar et al. (1996) screened the entire ICL3 region via deletion mutagenesis in three- or four-residue sections but found no effect on GLP-1 action, whereas Underwood et al. (2013) found that C341 ${ }^{\text {ICL3 }}$-A also had no effect (Takhar et al., 1996; Underwood et al., 2013).

xi. TM6 (D344 $\left.{ }^{6.33 b}-F 369^{6.58 b}\right)$. Takhar's ICL3 Ala screening also extended into the first eight residues of TM6, but no significant effect on GLP-1 action was shown (Takhar et al., 1996). Whereas Underwood et al. (2013) confirmed that $\mathrm{C} 347^{6.36 \mathrm{~b}} \mathrm{~A}$ also had no effect, Heller et al. (1996) showed that $\mathrm{R} 348^{6.37 \mathrm{~b}} \mathrm{G}$ resulted in a 12 -fold reduced affinity and inability to produce cAMP. T353 ${ }^{6.36 \mathrm{~b}} \mathrm{~A}$ led to a 22 -fold reduction in potency $\left(\Delta \log \tau_{c}=0.84\right.$ ) (Wootten et al., 2013c). The Ala substitution at E364 ${ }^{6.53 b}$ caused a 58-fold reduction in affinity with a 15 -fold reduced potency (Coopman et al., 2011), whereas the individual substitutions of E364 ${ }^{6.53 \mathrm{~b}}$ with Tyr, Asp, or Gln did not affect GLP-1 affinity or potency (Yang et al., 2016). Interestingly, the E364 ${ }^{6.53 b} \mathrm{Q}$ mutation resulted in a 50-fold and a 40 -fold increase in GLP-1- and exendin-4-binding affinity, respectively, in exendin 9-39 $_{\text {radioligand competition studies }}$ (Yang et al., 2016). Mutation of $\mathrm{H} 363^{6.52 \mathrm{~b}}$ to Ala resulted in either a 100-fold reduced affinity with no detectable potency (Coopman et al., 2011) or a 23 -fold reduced affinity with poor coupling $\left(17 \% \mathrm{E}_{\max }, \Delta \log \tau_{\mathrm{c}}=1.71\right)$. Mutation of F367 ${ }^{6.56 \mathrm{~b}}$ into Ala, Ile, and His decreased GLP-1 affinity and potency by 72 -fold, 20 -fold, and 131-fold, respectively (Yang et al., 2016).

xii. ECL3 (V370-G377). Residues from M371 ${ }^{\mathrm{ECL} 3}$ to G37 ${ }^{\text {ECL3 }}$ have been mutated to Ala (with A376 to Gly), showing only a modest effect upon GLP-1 pharmacology (Dods and Donnelly, 2015). D372 ${ }^{\mathrm{ECL} 3} \mathrm{~A}$ displayed wildtype affinity but a 60 -fold reduced potency, although analysis with the operational model suggested no effect upon efficacy; hence, this is likely to be the result of reduced receptor expression (13\% wild-type). $\mathrm{A} 375^{\mathrm{ECL} 3} \mathrm{G}$ resulted in a 10 -fold reduced affinity for GLP-1. ECL3 appears to play a role in ligand and/or ECD recognition in GCGR (Koth et al., 2012).

xiii. TM7 (Y378 $\left.{ }^{7.33 b}-Y 402^{7.57 b}\right)$. Residues from T378 ${ }^{7.33 b}$ to $\mathrm{E} 387^{7.42 \mathrm{~b}}$ have been mutated to Ala, suggesting that several residues play no major role in GLP-1 recognition-

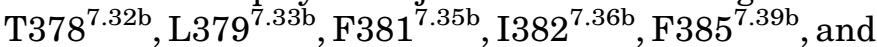
T386 ${ }^{7.40 \mathrm{~b}}$ (Dods and Donnelly, 2015). However, Moon et al. (2015) showed that, although $\mathrm{F} 381^{7.36 \mathrm{~b}} \mathrm{R}$ had no effect, $\mathrm{F} 381^{7.36 \mathrm{~b}} \mathrm{E}$ reduced potency and affinity by $>200$ fold, and L379 ${ }^{7.34 b} \mathrm{R}$ and $\mathrm{L} 379^{7.34 \mathrm{~b}} \mathrm{E}$ resulted in a more than 10-fold reduced affinity and in the range of 150 -fold decreased potency. $\mathrm{R} 380^{7.35 \mathrm{~b}} \mathrm{~A}$ resulted in 128 -fold reduced affinity, without reduced efficacy (Dods and Donnelly, 2015). The $R 380^{7.35 b} \mathrm{Q}$ substitution resulted in a more than 15-fold reduction in GLP-1 affinity and abolished potency (Yang et al., 2016), whereas $R 380^{7.35 b} \mathrm{D}$ lowered potency by 1850 -fold, but affinity was only reduced by 21 -fold, and $R 380^{7.35 b} \mathrm{G}$ decreased potency by 40 -fold and affinity by fourfold. K383 ${ }^{7.38 \mathrm{~b}} \mathrm{~A}$ showed no significant change in agonist affinity, but displayed reduced potency and efficacy (56-fold reduced potency, $\Delta$ Log $\tau_{\mathrm{c}}=1.18$ ) (Dods and Donnelly, 2015). Mutation of Y $402^{7.57 b} \mathrm{~A}$ demonstrated a role for this residue in coupling because substitution to Ala resulted in a large decrease in efficacy ( $\Delta \log \tau_{\mathrm{c}}=1.59$ ) (Wootten et al., 2013c), whereas the equivalent mutation in GIPR, Y392 ${ }^{7.57 b} \mathrm{~A}$, caused a fivefold reduction in potency (Yaqub et al., 2010). Substitutions at E387 $7.42 \mathrm{~b}, \mathrm{~T} 391^{7.46 \mathrm{~b}}, \mathrm{Q} 394^{7.49 \mathrm{~b}}, \mathrm{M} 397^{7.52 \mathrm{~b}}$, and C403 ${ }^{7.58 b}$ to Ala did not affect GLP-1 pharmacology (Coopman et al., 2011; Dong et al., 2012; Underwood et al., 2011; Wootten et al., 2013c). Substitution of L384 ${ }^{7.39}$ (Ala, Val) and L388 $8^{7.43}$ (Ala, Ile) into other aliphatic residues diminished GLP-1 affinity and potency in a similar way as corresponding mutants of homologous L382 $2^{7.39 b}$ and L386 $6^{7.43 b}$ residues in GCGR. The L386 ${ }^{7.43 b} \mathrm{~F}$ did not affect GLP-1R affinity or potency, whereas the corresponding L386 ${ }^{7.43 b} \mathrm{~F}$ mutant of GCGR abolished glucagon affinity and potency (Siu et al., 2013; Yang et al., 2016). The E387 ${ }^{7.42 b} \mathrm{~N}$ mutant did not affect GLP-1 binding or potency, whereas the E387 $7^{7.42 \mathrm{~b}} \mathrm{D}$ mutant decreased GLP-1 affinity and potency by 13 -fold and 10-fold, respectively, and the double E $364^{6.53 \mathrm{~b}} \mathrm{~N} / \mathrm{E} 387^{7.42 \mathrm{~b}} \mathrm{Q}$ mutant completely abolished GLP-1 binding (Yang et al., 2016). The reciprocal Ser ${ }^{8}$ Ala substitution of glucagon restored binding of the GLP-1R-mimicking D385 ${ }^{7.42 b} \mathrm{E}$ mutant of GCGR (Runge et al., 2003a), indicating that this receptorligand residue pair plays an important role in ligand selectivity between GLP-1R and GCGR.

xiv. $C$ terminus (C404-S463). The mutations of N406-A, R421-Q, C438-A, C458-A, and C462-A had no major effect on GLP-1 action (Koole et al., 2011; Underwood et al., 2013; Wootten et al., 2013c). Widmann et al. (1996a) investigated the role of serine residues (four serine doublets in the C-terminal tail) in receptor desensitization. Single- and double-Ala mutations of the doublets S431/S432, S441/S442, S444/S445, and S451/ S452 were analyzed to demonstrate their role in receptor phosphorylation and phorbol-12-myristate-13-acetateinduced desensitization (Widmann et al., 1996b).

\section{Receptor Function}

As a member of class B GPCRs, GLP-1R is highly conserved across species, thus underlining the physiologic importance (Huang et al., 2012). GLP-1R mediates the actions of GLP-1 via the incretin axis that is the 
functional connection between the intestine and the islets of Langerhans in the pancreas. Stimulation of the GLP-1R with GLP-1 primarily triggers the insulin release from islet $\beta$ cells in a glucose-dependent manner and suppresses glucagon secretion from islet $\alpha$ cells, in addition to several other effects such as delay of gastric emptying and inhibition of appetite (Koole et al., 2013a; II. Glucagon-Like Peptide-1).

\section{Signaling.}

a. Recombinant cells. The physiologic effects of GLP-1 are mediated by its interaction with GLP-1R and subsequent activation of its downstream signaling pathways. GLP-1R is pleiotropically coupled and signals through G-protein-dependent and independent mechanisms. It couples to G $\alpha$ s-elevating cAMP when overexpressed in recombinant cell lines, including CHO, HEK cells, Chinese hamster lung (CHL) fibroblasts, and COS cells (Wheeler et al., 1993; Widmann et al., 1994; Montrose-Rafizadeh et al., 1999; Wootten et al., 2013b). Increased calcium mobilization has also been observed in CHO, HEK, and COS, but not CHL cells (Wheeler et al., 1993; Widmann et al., 1994; Montrose-Rafizadeh et al., 1999; Coopman et al., 2010; Koole et al., 2010). The mechanism behind this, however, is controversial. Azidoanilide-GTP cross-linking provided evidence for GLP-1R activation of $\mathrm{G} \alpha \mathrm{q} / 11$ in $\mathrm{CHO}$ cells, whereas in HEK cells GTP $\gamma \mathrm{S}$ binding and immunoprecipitation studies showed no activation of $\mathrm{G} \alpha_{\mathrm{q}}$ or $\mathrm{G} \alpha_{\mathrm{i}}$ (Montrose-Rafizadeh et al., 1999; Coopman et al., 2010). Furthermore, although Wheeler et al. observed a rapid increase in inositol trisphosphates, corresponding with increased calcium levels, and suggesting phospholipase $\mathrm{C}$ activation in GLP-1R/COS cells, these intermediates did not seem to be involved in other studies with COS and HEK cells (Wheeler et al., 1993; Widmann et al., 1994; Coopman et al., 2010).

Azidoanilide-GTP cross-linking developed by MontroseRafizadeh and colleagues also revealed GLP-1R activation of $\mathrm{G} \alpha \mathrm{i} 1,2$ but not $\mathrm{G} \alpha \mathrm{i} 3$ in CHO cells. In this cell line, GLP-1 also activates MAPKs, including ERK1/2 (MontroseRafizadeh et al., 1999; Koole et al., 2010), and p38, through a cholera toxin-dependent pathway (MontroseRafizadeh et al., 1999). GLP-1R can also elicit G-proteinindependent signaling, via recruitment of the regulatory/ scaffolding $\beta$-arrestin proteins (Sonoda et al., 2008; Quoyer et al., 2010). $\beta$-arrestin 2 recruitment has been observed in CHO, HEK293, and COS-7 cells, and $\beta$-arrestin 1 in CHO cells (Jorgensen et al., 2005; Schelshorn et al., 2012; Wootten et al., 2013b).

b. Pancreatic $\beta$ cells. Research has largely focused on characterizing the GLP-1R signaling in $\beta$ cells, where it mediates increased insulin secretion, storage, and synthesis as well as increased $\beta$ cell mass. Consistent with the findings in recombinant cells, elevated cAMP is vital in $\beta$ cells for glucose-dependent insulin secretion mediated by GLP-1R, and, although calcium mobilization, phosphorylation of ERK, and $\beta$-arrestin are important, $\mathrm{G} \alpha \mathrm{q} / 11$ does not seem to play a major role. A summary of known pathways involved in pancreatic $\beta$ cell function is illustrated in Fig. 4.

i. Insulin Secretion. $\quad \beta$ cells undergo glucose-stimulated insulin secretion. Glucose enters the $\beta$ cell through glucose transporter 2 and is converted through glycolysis to pyruvate, which enters the mitochondria for oxidative phosphorylation, increasing the cytosolic ATP/ADP ratio (MacDonald et al., 2005). The increased ATP closes $K_{\text {ATP }}$ channels, depolarizing the plasma membrane and increasing calcium influx through L-type voltage-dependent calcium channels, causing release of calcium from intracellular stores through calcium-induced calcium release (CICR) (MacDonald et al., 2005). Increased cytoplasmic calcium stimulates exocytosis of the insulin secretory granules.

GLP-1R signaling enhances this glucose-dependent insulin secretion through activation of $\mathrm{G} \alpha \mathrm{s}$, upregulation of cAMP, and subsequent activation of PKA and exchange protein activated by cAMP (Epac). PKA is a holoenzyme composed of both regulatory and catalytic subunits; the latter are released upon binding of cAMP to the regulatory subunits, leading to phosphorylation of downstream substrates, whereas Epac2 primarily functions as a guanine nucleotide exchange factor for small Ras-like G proteins, thereby regulating their activity. PKA phosphorylates the sulfonylurea receptor (SUR1) subunit of the $\mathrm{K}_{\mathrm{ATP}}$ channels, closing them and further depolarizing the membrane (Light et al., 2002). Epac also inhibits $\mathrm{K}_{\mathrm{ATP}}$ by increasing its sensitivity to ATP (Kang et al., 2008). The cAMP/PKA pathway, in concert with the PI3K/protein kinase $\mathrm{C}$ (PKC) $\zeta$ pathway (discussed in more detail below), also inhibits voltagegated potassium channels, which in response to depolarization, opens and allows $\mathrm{K}^{+}$efflux, repolarizing the cell (MacDonald et al., 2003). This delays repolarization, allowing increased calcium influx via voltagedependent calcium channels (MacDonald et al., 2003). PKA and Epac1/2 are also involved in CICR from the ER, which is mediated by PKA via the inositol 1,4,5trisphosphate receptor and by Epac1/2 through ryanodine receptors, increasing intracellular calcium (Kang et al., 2003; Tsuboi et al., 2003; Dyachok and Gylfe, 2004). These mechanisms, therefore, enhance the ability of $\beta$ cells to exocytose insulin secretory granules. There is evidence that CICR also contributes to increased mitochondrial ATP, in conjunction with GLP-1induced increases in ATP (Tsuboi et al., 2003). It was proposed that the intracellular calcium may enter the mitochondria to activate mitochondrial dehydrogenases to increase ATP production (Tsuboi et al., 2003). PKA and Epac also seem to have a role in the exocytosis of insulin secretory granules, with PKA phosphorylating snapin, a protein that is vital to the regulation of vesicle assembly, and Rab-3-interacting molecule (Rim) and Munc-131, which are involved in vesicle fusion (Kwan et al., 2007; Song et al., 2011). Epac2 also interacts with 


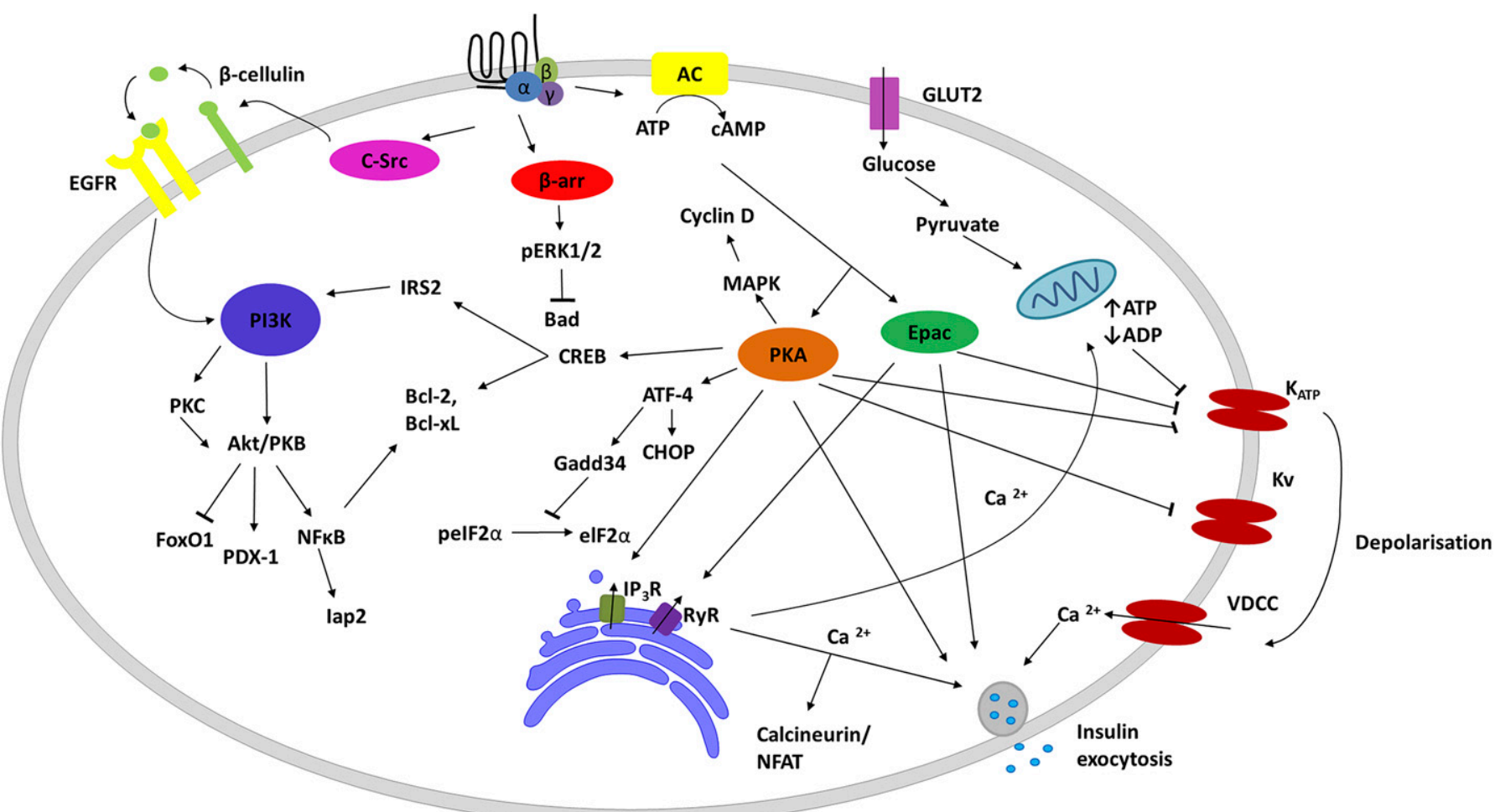

Fig. 4. Summary of the main characterized pathways of glucose and GLP-1 signaling in the pancreatic $\beta$ cell. Glucose enters the cell through glucose transporter 2 and undergoes glycolysis to produce pyruvate that enters the mitochondria for oxidative metabolism and ATP production. This increase in cytosolic ATP closes the $\mathrm{K}_{\mathrm{ATP}}$ channels, depolarizing the membrane and opening the voltage-dependent calcium channels, increasing calcium influx into the cell, causing insulin exocytosis. GLP-1 increases insulin exocytosis through a number of mechanisms. GLP-1R couples to G $\alpha$ s, activates adenylate cyclase that converts ATP to cAMP, and mobilizes two downstream effectors, PKA and Epac. These have a range of effects, including closing $\mathrm{K}_{\mathrm{ATP}}$ channels, enhancing fusion of insulin secretory granules with the membrane, whereas PKA also closes Kv channels, inhibiting membrane repolarization. PKA and Epac also increase intracellular calcium by facilitating CICR through the opening of inositol 1,4,5-trisphosphate receptor and ryanodine receptor calcium channels, respectively. This increase in calcium has also been proposed to upregulate mitochondrial ATP production and activate calcineurin, so nuclear factor of activated T cells promotes insulin gene transcription to increase insulin stores. Alongside increasing insulin synthesis and exocytosis, GLP-1 signals through a number of pathways to increase $\beta$ cell mass. PKA reduces ER stress through ATF-Gadd34 signaling, increases $\beta$ cell neogenesis by activating cyclin $\mathrm{D}$, and elevates the expression of insulin receptor substrate 2 (IRS2), a $\beta$ cell survival factor, as well as anti-apoptotic proteins Bcl-2 and Bcl- $x_{\mathrm{L}}$ through CREB. PI3K is activated by IRS2 and transactivation of epidermal growth factor receptor, and this further promotes increased $\beta$ cell mass through upregulation of PDX-1 and nuclear factor $\kappa \mathrm{B}$, which upregulates anti-apoptotic Bcl-2 /Bcl- $\mathrm{x}_{\mathrm{L}}$ and inhibitor of apoptosis protein-2.

Rim2 and Piccolo, a calcium sensor, to form a complex that is important for insulin secretion (Fujimoto et al., 2002). Studies in islets of phospholipase $C$ and Epac knockout mice also suggest that Epac2 acts via Rap1regulated phospholipase $\mathrm{C} \varepsilon$ to cause calcium-induced insulin exocytosis (Shibasaki et al., 2007; Dzhura et al., 2011). GLP-1 enhances glucokinase activity through Epac2 in a Rim2/Rab3A-dependent manner, sensitizing $\beta$ cells to glucose. Knockdown of $\beta$-arrestin 1 in cultured pancreatic cells resulted in a reduction of both GLP1mediated cAMP production and insulin secretion (Sonoda et al., 2008). Evidence suggests that this occurs via an increase in $\beta$-arrestin 1 -mediated ERK1/2 phosphorylation that enhances cAMP response element-binding protein (CREB) activation and cAMP levels, subsequently contributing to insulin secretion (Quoyer et al., 2010).

ii. Insulin Synthesis and Storage. GLP-1 also acts to increase insulin stores in $\beta$ cells by promoting insulin gene transcription, its mRNA stability, and biosynthesis. This occurs in both cAMP/PKA-dependent and independent manners (Baggio and Drucker, 2007). Studies with the rat insulin I promoter have implicated basic region leucine zipper proteins, similar in structure to CREB, which bind to the cAMP-responsive element site to upregulate insulin transcription in a cAMP/ PKA-independent mechanism (Skoglund et al., 2000; Chepurny et al., 2002). The upregulation of the expression and activity of transcription factor pancreaticduodenum homeobox-1 (PDX-1) and its increased activity also promote insulin transcription and biosynthesis via a PKA-mediated mechanism (Wang et al., 1999, 2001). Glucose and GLP-1 potentiate insulin gene transcription, by increasing calcium levels, activating calcineurin that dephosphorylates nuclear factor of activated $\mathrm{T}$ cells, resulting in its nuclear localization and the promotion of insulin gene transcription (Lawrence et al., 2002).

iii. $\beta$ Cell Survival, Proliferation, and Neogenesis. GLP-1 promotes $\beta$ cell proliferation, neogenesis, and inhibition of apoptosis in rat models of T2DM (Buteau, 2011). GLP-1R activation facilitates the release of $\beta$-cellulin by membrane-bound metalloproteinases, inducing transactivation of epidermal growth factor receptor, which then signals through PI3K and activates 
Akt/protein kinase $\mathrm{B}(\mathrm{PKB}), \mathrm{PKC} \zeta$, and p38 MAPK and ERK downstream (Buteau, 2011).

PDX-1 is also critical in increasing $\beta$ cell mass due to its proliferative and anti-apoptotic effects ( $\mathrm{Li}$ et al., 2005). As well as the increased activity mentioned above, GLP-1R enhances its expression. Akt inhibits forkhead transcription factor (Foxo1) action through its nuclear exclusion, relieving its inhibition on expression of forkhead box protein A2, which controls PDX-1 expression (Kitamura et al., 2002; Buteau et al., 2006). In addition, GLP-1-mediated activation of PKA upregulates CREB, increasing the expression of IRS2, a $\beta$ cell survival factor, leading to activation of PI3K/PKB and the anti-apoptotic protein Bcl-2 (Wilson et al., 1996; Rhodes and White, 2002; Jhala et al., 2003). Bcl-2 and Bcl- $\mathrm{x}_{\mathrm{L}}$ are also upregulated through the activation of nuclear factor $\kappa \mathrm{B}$ downstream of PKB (Buteau et al., 2004). Nuclear factor $\kappa \mathrm{B}$ increases expression of inhibitor of apoptosis protein-2, thereby preventing apoptosis (Buteau et al., 2004). GLP-1 signaling has also been implicated in downregulation of proapoptotic caspase-3 and decrease in the cleavage of poly-ADPribose (Hui et al., 2003; D'Amico et al., 2005). ERK1/2 signaling via $\beta$-arrestin 1 also activates p90RSK, which phosphorylates proapoptotic $\mathrm{Bad}$ and inactivates it (Quoyer et al., 2010).

PKA activation of MAPK and cyclin D1 is important in the transition of the G1/Gs phase essential to cell cycle progression: they promote $\beta$ cell neogenesis (Friedrichsen et al., 2006). GLP-1R agonists also improve $\beta$ cell function and survival upon ER stress. This is believed to occur through PKA activation of C/EBP homologous protein and growth arrest and DNA damage-inducible protein (Gadd34), which prevents the dephosphorylation of translation initiator elF2 $\alpha$, allowing the ER to recover from stress (Yusta et al., 2006).

c. Extrapancreatic signaling. Although GLP-1 signaling mediates many other physiologic effects, and GLP-1R is expressed in extrapancreatic tissues, the mechanistic basis for these effects is less well characterized. Therefore, there is limited knowledge regarding the underlying signaling mechanisms, although some important pathways and molecules have been identified in a limited subset of tissues.

$i$. Liver. GLP-1 reduces hepatic gluconeogenesis and lipogenesis and increases glycogen formation, but there is some debate over whether these effects are mediated by GLP-1R in hepatocytes, or whether the effects may be indirectly mediated through CNS or insulin release. GLP-1 promotes glycogen synthesis and decreased gluconeogenesis in vitro through upregulation of glycogen synthase that occurs downstream of PI3K/PKB, $\mathrm{PKC}$, and serine/threonine protein phosphatase 1 , and also by reduced expression of gluconeogenetic enzyme phosphoenol pyruvate carboxykinase in rat hepatocytes (Redondo et al., 2003; Raab et al., 2009). In this system, GLP-1 also increases the phosphorylation of MAPK and p70s6 $\mathrm{k}$ that perhaps are involved in other GLP-1 effects (Redondo et al., 2003). However, in both studies, the presence of GLP-1R in these hepatocytes was not confirmed.

Signaling mechanisms for fatty acid oxidation and insulin sensitization, important in reducing hepatic steatosis, however, have been characterized in rat hepatocytes confirmed to express GLP-1R (Svegliati-Baroni et al., 2011). Exendin-4 treatment resulted in increased peroxisome proliferator-activated receptor (PPAR) activity and PPAR $\gamma$ expression through the activation of PI3K and $5^{\prime}$ AMP-activated protein kinase (AMPK) pathways (Svegliati-Baroni et al., 2011). Increased PPAR activity induced the transcription of fatty acid $\beta$-oxidizing enzymes such as acyl-coenzyme A oxidase 1-palmitoyl and carnitine palmitoyltransferase $1 \mathrm{~A}$, thereby reducing fatty acid levels in hepatocytes (Svegliati-Baroni et al., 2011). Increased PPARy expression allowed increased insulin sensitization through the reduction of $\mathrm{Ser}^{307}$ c-Jun N-terminal kinase phosphorylation (Svegliati-Baroni et al., 2011). GLP-1R activation also seems to reduce insulin resistance by intersecting the insulin-signaling pathway. In HepG2 and Huh7 cells expressing GLP$1 \mathrm{R}$, exendin-4 treatment led to the phosphorylation of key mediators of the insulin-signaling pathway; PDX1, Akt1, and PKC $\zeta$ and small interfering RNA of GLP1R knocked down this phosphorylation for PDX-1 and PKC $\zeta$ (Gupta et al., 2010).

ii. Kidney. GLP-1R activation in the kidney mediates natriuretic and diuretic effects of GLP-1, including decreasing renal proximal tubule reabsorption, improving endothelial integrity, and reducing hypertension. The transporter NHE3, which largely mediates this reabsorption, is inhibited by GLP-1 through PKA and Epac. Exendin-4 causes decreased NHE3 function in the porcine kidney epithelial cell line, LLC-PK(1), with phosphorylation at serine 552, a PKA consensus site. The mechanism by which Epac inhibits the NHE3 transporter has not been determined (Carraro-Lacroix et al., 2009). It is known that angiotensin II signaling increases NHE3 activity, and oxidative stress is inhibited by GLP-1R-mediated cAMP elevation. In glomerular endothelial cells, exendin-4 promoted PKA-dependent phosphorylation of $\mathrm{c}-\operatorname{Raf}\left(\operatorname{Ser}^{259}\right)$, preventing the activation of the angiotensin IIdependent pathway, p-c-Raf(Ser $\left.{ }^{338}\right) / E R K 1 / 2 /$ plasminogen activator inhibitor-1, a pathway upregulated in diabetes due to a hyperglycemic increase in PKC- $\beta$ (Mima et al., 2012).

GLP-1R may also mediate renal protective effects through a reduction of PPAR $\alpha$ as exendin- 4 treatment decreased PPAR $\alpha$ expression in both $d b / m$ and $d b / d b$ mouse models (Park et al., 2007). This decreased expression of PPAR $\alpha$ was paralleled by decreased transforming growth factor- $\beta 1$, decreased type IV collagen, decreased caspase-3 expression, and reduced mesangial expansion (Park et al., 2007). 
iii. Adipocytes. GLP-1R signaling has been implicated in increased glucose uptake in human adipocytes, believed to be mediated by PI3K and MAPK (Sancho et al., 2007). GLP-1R also promotes increased adipocyte mass through preadipocyte proliferation and inhibition of apoptosis, mediated by ERK-, PKC-, and AKT-signaling pathways (Challa et al., 2012).

iv. Nervous System. GLP-1R expressed in the nucleus tractus solitarius signals to suppress food intake and reduce body weight, and this is proposed to be mediated through PKA (Hayes et al., 2011). PKA decreases phosphorylation of AMPK and increases phosphorylation of $\mathrm{p} 44 / 23 \mathrm{MAPKs} / \mathrm{mitogen}$-activated protein kinase (MEK) (Hayes et al., 2011).

v. Cardiovascular System. GLP-R is thought to confer cardioprotection through a number of mechanisms, including reducing damage caused by ischemia via inhibiting apoptosis and through oxidative stress, and improving energy utilization. GLP-1 is able to reduce infarct size in rat hearts, an effect that is attenuated by inhibition of cAMP, PI3K, and p42/44 MAPK and GLP-1R, and also requires activation of the $\mathrm{mTOR} / \mathrm{p} 70 \mathrm{~s} 6$ kinase pathway (Bose et al., 2005b, 2007). Murine studies have implicated additional important signaling molecules. The GLP-1Rdependent cardioprotective effects of liraglutide in mouse cardiomyocytes were associated with an elevation of cAMP and increased caspase-3 activity (Noyan-Ashraf et al., 2009). In addition, liraglutide-mediated suppression of glycogen synthase kinase $3-\beta$ (GSK- $3 \beta$ ) and caspase-3 activation in murine hearts was not evident in GLP-1R knockout mice (Noyan-Ashraf et al., 2009), confirming that these effects are receptor-dependent. Stimulation of GLP$1 \mathrm{R}$ also decreases $\mathrm{H}_{2} \mathrm{O}_{2}$-induced production of reactive oxygen species and upregulation of antioxidant enzymes in an Epac-dependent manner (Mangmool et al., 2015).

GLP-1 may signal via AMPK and Akt to improve glucose metabolism after an injury to promote recovery. These were important mediators upon exenatide treatment of TG9 mice that caused improved cardiac contractility, elevated myocardial GLUT4 expression, and increased uptake of 2-deoxyglucose (Vyas et al., 2011).

2. Ligand-Directed Signal Bias. As described above, GLP-1R is pleiotropically coupled to a range of signaling effectors, each of which can impact on the physiologic response elicited by receptor activation. This allows the potential for individual ligands to evoke different patterns of response upon interaction with the receptor in what is termed ligand-directed biased signaling (Shonberg et al., 2014). Such biased signaling and regulation are thought to arise through the stabilization of distinct ensembles of receptor conformations that occur through the varying chemical contacts between ligands and the receptor. Although gross changes in signal bias can be recognized as a reversal of potency or efficacy (Kenakin and Miller, 2010; Koole et al., 2013b; Shonberg et al., 2014), less dramatic effects require a quantitative framework to identify significant differences in ligand response. The most robust method to quantify efficacy is the operational model of Black and Leff (Kenakin and Christopoulos, 2013 ), with the transduction ratio of $\tau / \mathrm{K}_{\mathrm{A}}$ used to define strength of signaling of an individual ligand for a specific pathway, and this ratio can be determined from classic concentration-response experiments (Kenakin et al., 2012).

a. Peptide-mediated signal bias. There are multiple endogenous peptides that can interact with and activate GLP-1R, including the fully processed GLP-1 $1_{7-36}$ amide and GLP-1 $1_{7-37}$ peptides, the extended 1-36 and 1-37 forms of these peptides, as well as oxyntomodulin. The search for more stable forms of GLP-1 has also provided a range of mimetic peptides, including exendin and modified forms of GLP-1 that have been developed for therapeutic use (V. Pharmaceutical Development and Therapeutics). Evidence for biased signaling requires measurement of multiple signaling endpoints. Early work characterizing ligands for GLP-1R was largely limited to measurement of cAMP, as the latter is the most well-coupled pathway and critical for the incretin effect on $\beta$ cells. Therefore, it is only relatively recently that evidence for ligand-directed signaling has emerged.

Initial studies used heterologously expressed GLP-1R to more broadly examine three canonical pathways that have been linked to physiologic signaling in $\beta$ cells, specifically cAMP accumulation, ERK phosphorylation, and intracellular calcium $\left(\mathrm{iCa}^{2+}\right)$ mobilization. Even with this relatively limited assessment of signaling, quantitative evidence for peptide-mediated signal bias was observed, with oxyntomodulin exhibiting a relative bias toward pERK over cAMP and $\mathrm{iCa}^{2+}$ signaling compared with either GLP-1 or exendin (Koole et al., 2010), and this supported earlier observations of differences in cAMP signaling and $\beta$-arrestin recruitment between oxyntomodulin and GLP-1 $1_{7-36}$ amide (Jorgensen et al., 2007). Likewise, the extended form of GLP-1 $1_{1-36}$ amide had a relative bias toward pERK, but a loss of $\mathrm{iCa}^{2+}$ signaling. Thus, this work demonstrated GLP-1R was subject to the potential for peptide-mediated biased signaling, although no significant bias was observed between amidated and nonamidated forms of GLP-1 $1_{7-36}$ or between GLP-1 and exendin for these pathways (Koole et al., 2010). More extensive analysis of signaling and regulatory pathways that included recruitment of arrestins revealed additional tiers of biased signaling with both exendin and oxyntomdulin exhibiting stronger arrestin recruitment relative to GLP-1 ${ }_{7-36}$ amide, whereas GLP-1 $1_{1-36}$ did not recruit arrestins (Wootten et al., 2013b) (Fig. 5, left panel), at least in this recombinant system. The synthetic 11-mer peptide, BMS21, although much less potent than GLP- $1_{7-36}$ amide, had a relatively similar profile of activation of the canonical pathways (cAMP, pERK, and $\mathrm{iCa}^{2+}$ ), but did not elicit recruitment of arrestins (Wootten et al., 2013b). Intriguingly, the principal metabolite of GLP-1, GLP-1 $1_{9-36}$ amide, displays a relative preservation of $\mathrm{pERK}$ signaling 
(Li et al., 2012a; Wootten et al., 2012), despite a marked attenuation of cAMP production and ability to induce insulin secretion (Wootten et al., 2012), indicating that this peptide is strongly biased toward pERK and may be capable of signaling in a physiologically relevant manner. Additional evidence for peptide-mediated signaling bias is seen with a yeast assay of chimeric $G$ protein activation (Weston et al., 2014), consisting of the yeast $\mathrm{G}$ protein GPA1 substituted by five amino acids from the human $\mathrm{G} \alpha$ sequence. In this system, exendin, oxyntomodulin, and glucagon exhibited relative bias toward the GPA1/G $\alpha$ i chimera over the GPA1/G $\alpha \mathrm{S}$ chimera when compared with signaling by GLP-1 $1_{7-36}$, whereas liraglutide had a similar profile of signaling to GLP-1 across these two pathways (Weston et al., 2014). This indicates that biased signaling occurs even at the level of $G$ protein recruitment and is consistent with stabilization of different ensembles of receptor conformations by different peptide ligands.

$b$. Nonpeptide-mediated bias. There has been considerable interest in the development of nonpeptidic ligands for GLP-1R as leads with improved bioavailability (Chen et al., 2007; Knudsen et al., 2007; Willard et al., 2012b). Although these ligands are principally tested in assays of cAMP formation and insulin secretion, a number of these compounds have now been evaluated across a more broad range of pathways, enabling assessment of the extent to which these compounds exhibit bias relative to native GLP-1 signaling. The most extensively studied are the Eli Lilly compound, 4-(3-benzyloxyphenyl)2-ethylsulfinyl-6-(trifluoromethyl)pyrimidine (BETP), and the Novo Nordisk compound 2 (Coopman et al., 2010; Koole et al., 2010; Cheong et al., 2012; Willard et al., 2012a; Wootten et al., 2013b), but there are now some data comparing signaling for Boc5 and the TransTech Pharma compound TT15 (Wootten et al., 2013b). Although these compounds have low potency for cAMP production, relative to native GLP- $1_{7-36}$ amide, they also exhibit distinct patterns of signal bias (Koole et al., 2010; Wootten et al., 2013b). This can be illustrated in a web of bias (Fig. 5, right panel), which reveals that Boc5 and TT15 have similar patterns of signaling via GLP-1R for canonical pathways (cAMP, pERK, and $\mathrm{iCa}^{2+}$ ), but have diminished ability to recruit arrestin proteins. In contrast, compound 2 and BETP have a relatively enriched ability to recruit arrestins, but distinct effects on pERK and $\mathrm{iCa}^{2+}$ mobilization, where BETP trends towards reduced $\mathrm{pERK}$ but higher $\mathrm{iCa}^{2+}$, and compound 2 has the opposite profile (compared to the reference cAMP pathway and the signaling profile of GLP-1). Additional differences in the behavior of compound 2 and/or BETP have also been noted by others (Coopman et al., 2010; Cheong et al., 2012; Thompson and Kanamarlapudi, 2015), albeit that a lack of a quantitative framework for these analyses meant that potential implications for signaling bias were not fully explored. The fact that these nonpeptidic compounds have distinct profiles of signaling is not surprising, as their chemical diversity means that they interact with the receptor very differently from peptidic ligands. Indeed, BETP and compound 2 interact with the intracellular face of GLP-1R in a manner that involves covalent modification of C347 in ICL3/TM6 (Nolte et al., 2014).

3. Molecular Basis for Signal Bias. There is increasing evidence demonstrating that the conformational change that drives activation transition involves reordering of hydrogen bond networks and intrahelical packing. Such a change promotes reorganization of the intracellular face of the receptor, thereby enabling interaction with intracellular effectors (Zhou et al., 2000; Curran and Engelman, 2003; Angel et al., 2009; Illergard et al., 2011). These networks are relatively well conserved within GPCR subfamilies and involve conserved polar residues (Venkatakrishnan et al., 2013). This is best studied for class A GPCRs, and suggests that there is evolutionary conservation of the mechanisms underpinning activation transition. Although there is limited direct homology in the amino acid sequence of the TM domains between the major receptor families, it is expected that such conserved polar residues within class B GPCRs play a similarly important structural role and contribute to the mechanism of ligand-directed biased signaling. This view is supported by experiments in which conserved intramembranous polar residues in GLP-1R were mutated to alanine. A cluster of residues, including $\mathrm{R} 190^{2.60 \mathrm{~b}}$, $\mathrm{N} 240^{3.43 \mathrm{~b}}$, $\mathrm{H} 363^{6.52 \mathrm{~b}}$, and Q394 ${ }^{7.49 \mathrm{~b}}$, when mutated, was shown to change the signaling profile of the receptor for cAMP, $\mathrm{pERK}$, and $\mathrm{iCa}^{2+}$ in both a residue-specific and ligand-specific manner (Wootten et al., 2013c). Although differences were particularly marked between GLP-1 and oxyntomodulin, selective differences in the effect of mutation were also seen between GLP-1 and exendin, providing molecular evidence that the mechanism of receptor activation differs between these two peptides, despite relatively similar overall efficacy for the peptides across these pathways at the wild-type receptor (Wootten et al., 2013c). In addition to these residues that displayed ligand-specific effects, there was a series of serine residues $\left(\mathrm{S} 155^{1.50 \mathrm{~b}}, \mathrm{~S} 186^{2.56 \mathrm{~b}}\right.$, and $\mathrm{S} 392^{7.47 \mathrm{~b}}$ ) that played a role in the control of receptor-dependent signal bias; however, these had global effects on all peptides (Wootten et al., 2013c). These residues sit at the interface between either TM1 and TM7 (S155 $5^{1.50 \mathrm{~b}}$ and S392 $2^{7.47 \mathrm{~b}}$ ) or TM2 and TM3 $\left(\mathrm{S} 186^{2.56 \mathrm{~b}}\right)$ and are most likely involved in tight packing of these helices. With the solution of crystal structures of the TM domain of the related $\mathrm{CRF}_{1}$ and glucagon receptors (Hollenstein et al., 2013; Siu et al., 2013; Jazayeri et al., 2016), it is now possible to model the location of these residues with improved precision. Intriguingly, such modeling indicates that these residues reside at a fulcrum position of the receptor TM 

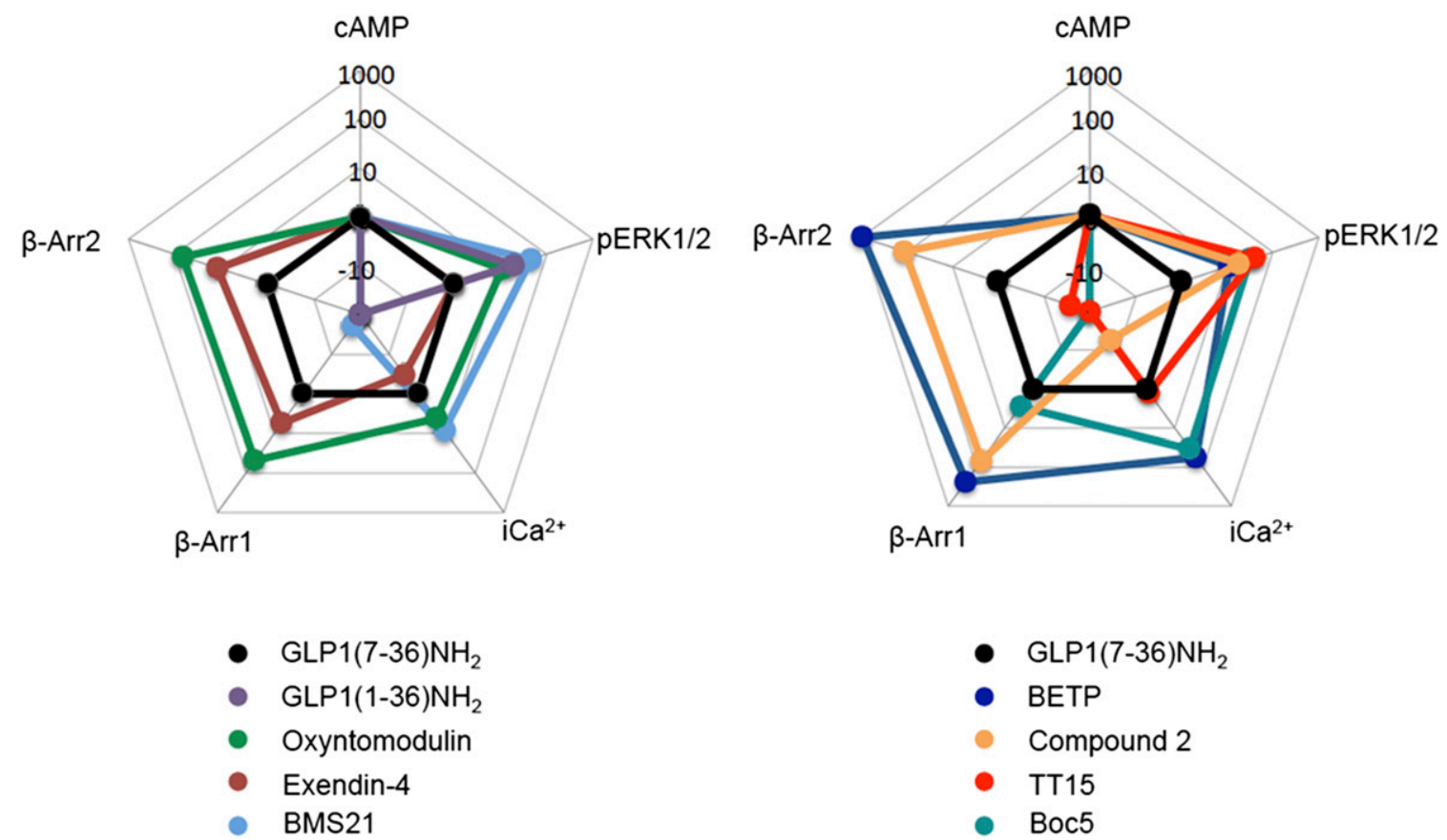

$\begin{array}{ll}\text { - } & \text { GLP1(7-36) } \mathrm{NH}_{2} \\ \text { - } & \text { BETP } \\ \text { - Compound } 2 \\ \text { - TT15 } \\ \text { - Boc5 }\end{array}$

Fig. 5. Web of bias illustrating distinctions in the pattern of signaling of different peptide agonists (left panel) or nonpeptidic modulators (right panel) at GLP-1R. The web of bias plots $\Delta \Delta \tau / \mathrm{K}_{\mathrm{A}}$ values on a logarithmic scale for each ligand and for every signaling pathway tested. Formation of these values included normalization to the reference ligand GLP-1 $1_{7-36}$ amide and the reference pathway, cAMP accumulation. The plots do not provide information on absolute potency, but on relative efficacy for signaling of individual pathways in comparison with that for cAMP. Data are from Koole et al., 2010; Willard et al., 2012a; and Wootten et al., 2013b.

bundle, where the splayed helices of the open extracellular face of the receptor converge (Fig. 6), with the residues that contribute to ligand-dependent signaling forming a central interaction network and the smaller polar residues that are globally important for signaling external to this core.

Although not broadly conserved across the class B subfamily, there is an additional polar threonine in TM1, at position $149^{1.44 b}$, that is the site of a naturally occurring polymorphism leading to incorporation of a methionine in this position (Beinborn et al., 2005). Methionine at this position leads to a marked loss in coupling of the receptor to cAMP accumulation (Fortin et al., 2010; Koole et al., 2011) and $\mathrm{iCa}^{2+}$ mobilization, whereas ERK phosphorylation is relatively preserved (Koole et al., 2011). This is a global effect for peptide activators of the receptor (Koole et al., 2011). Exploration of the tolerance of this position to different amino acids revealed that serine substitution had relatively minimal detrimental effect, suggesting that maintenance of the polar nature of this residue is important. However, amino acids of similar size, although leading to attenuation of response in a pathway-specific manner, were the least affected, indicating that side chain packing was also important (Koole et al., 2015). Thus, this mutation changes the physiologic bias of the receptor in addition to markedly attenuating peptide-mediated signaling.

Although these experiments have been principally interpreted in the context of in cis signaling of receptor to effectors, GLP-1R, like most, if not all class B GPCRs, undergoes functionally important dimerization (Harikumar et al., 2006, 2010, 2012; Gao et al., 2009; Schelshorn et al., 2012). Intriguingly, disruption of the TM4 dimer interface of GLP-1R differentially impacted the signaling elicited by agonist peptides with a greater abrogation of $\mathrm{iCa}^{2+}$ mobilization relative to that of either cAMP accumulation or ERK phosphorylation (Harikumar et al., 2012). This indicates that differential ligand-mediated signaling could also involve the allosteric interaction of protomers within a receptor dimer.

In addition to an emerging appreciation of how ligands can selectively alter key hydrogen-bonding networks, there is some limited information on how extracellular loop residues contribute to peptide-mediated bias, in particular for ECL2 (Koole et al., 2012a,b). K288 ${ }^{\text {ECL2 }}$, $\mathrm{C} 296^{\mathrm{ECL} 2}, \mathrm{~W} 297^{\mathrm{ECL} 2}$, and N300 ${ }^{\mathrm{ECL} 2}$ had critical roles in governing signal bias of the receptor, but were also globally important for peptide signaling. Nonetheless, peptide-specific effects on relative efficacy and signal bias were most frequently observed for residues 301-305, although $\mathrm{R} 299^{\mathrm{ECL} 2} \mathrm{~A}$ mutation also exhibited different effects for individual peptides. M303 ${ }^{\text {ECL2 }}$ appeared to play a greater role for exendin and oxyntomodulin actions than those of GLP-1 peptides. Interestingly, ECL2 mutation was generally more detrimental to exendinmediated $\mathrm{iCa}^{2+}$ mobilization than GLP-1 ${ }_{7-36}$ amide, providing additional support for subtle variances in receptor activation by these two peptides.

4. Allosteric Modulation. As described above (II. Glucagon-Like Peptide-1 and III. Glucagon-Like Peptide 1 Receptor), peptide ligands engage GLP-1R via a diffuse pharmacophore that includes key interactions for affinity 

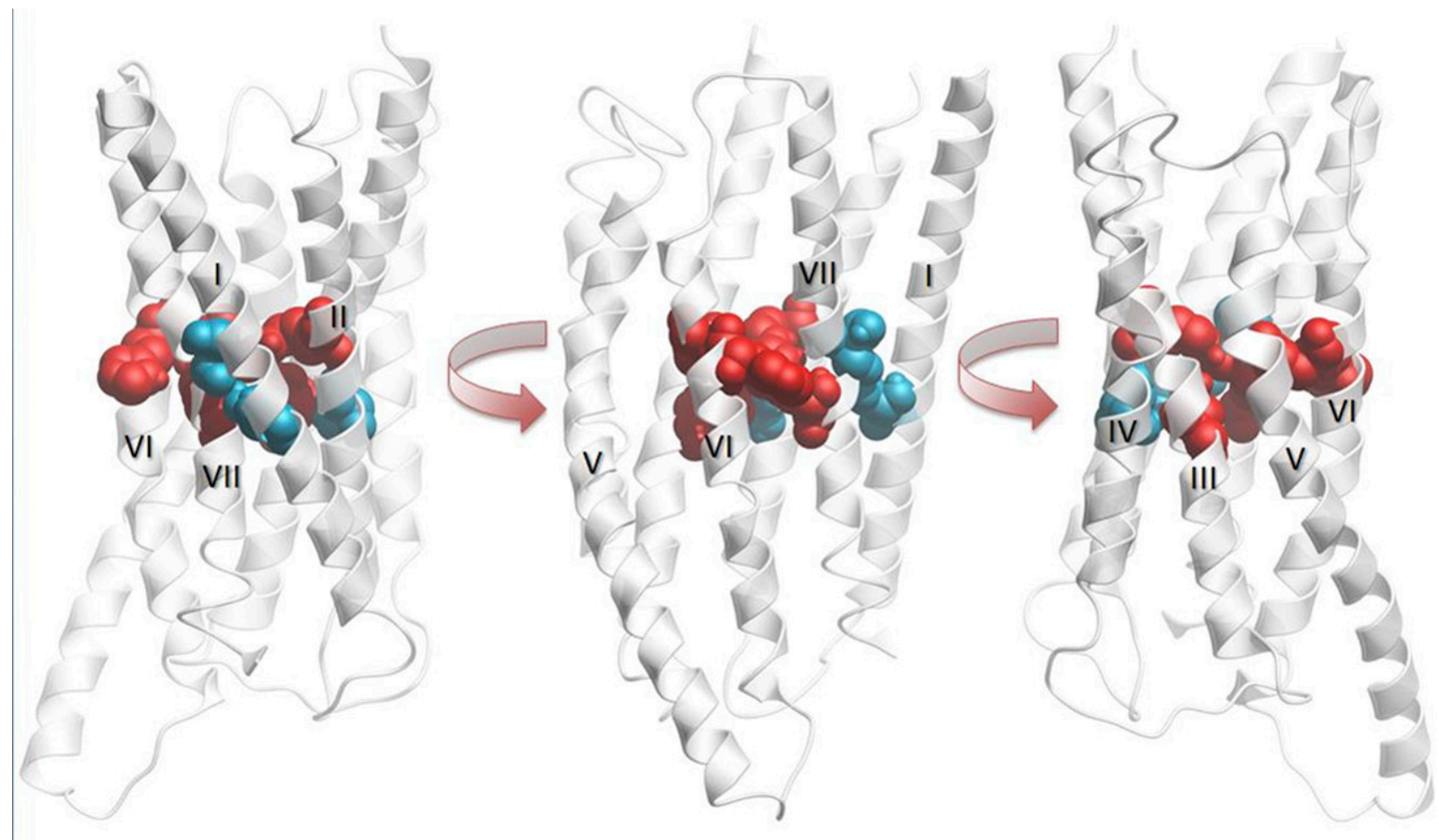

Fig. 6. Homology model of GLP-1R illustrating the relative position of key residues involved in the receptor-signaling bias. The modeling indicates that these residues reside at a fulcrum position of the receptor transmembrane bundle, where the splayed helices of the open extracellular face of the receptor converge, with the residues that contribute to ligand-dependent signaling forming a central interaction network (space fill, red) and the smaller polar residues that are globally important for signaling external to this core (space fill, blue). The receptor is displayed in three views at different horizontal rotation. Transmembrane helices are numbered with Roman numerals.

with the N-terminal extracellular domain, as well as poorly defined interactions with the extracellular loops and TM domain core that drive receptor activation. Nonpeptidic ligands have a distinct mode of binding, and in many cases can do so via topographically distinct, allosteric sites from those of the native peptides. Such ligands can bind simultaneously with peptide ligands and interact in a cooperative manner to alter the binding and/or efficacy of peptide signaling (and vice versa) (Leach et al., 2007; May et al., 2007; Keov et al., 2011; Wootten et al., 2013a; Gentry et al., 2015). Allosteric modulation of GPCRs is now a well-established paradigm that provides both advantages and challenges for drug discovery when compared with classic orthosteric ligands (Wootten et al., 2013a). The first characterized allosteric modulators of GLP-1R were identified by Novo Nordisk, and are exemplified by compound 2, which, as noted above, is an allosteric agonist of the receptor. In addition to its intrinsic efficacy, this series of compounds augmented the binding of radiolabeled GLP-1, indicating that it could act as a positive allosteric modulator (Knudsen et al., 2007). Nonetheless, there was only limited impact on the efficacy of GLP-1R for cAMP signaling. A hallmark of allosteric interactions is the phenomenon of probe dependence that describes the capacity for different effects, depending upon the orthosteric and allosteric ligand combination. This is also observed for ligands of GLP-1R. Although compound
2 has only limited effect on GLP- $1_{7-36}$ amide-induced cAMP production, it yields a $\sim 30$-fold augmentation of oxyntomodulin signaling via this pathway (Koole et al., 2010; Willard et al., 2012b; Wootten et al., 2013b). Probe dependence is also seen for effects on the extended GLP-1 peptides and exendin, where very limited augmentation of cAMP signaling is observed. A similar pattern of effect is observed for BETP in that this compound also augments oxyntomodulin signaling, but has minimal effect on GLP-1 $1_{7-36}$ amide - , exendin-, or GLP-1 $1_{1-36}$ amide ${ }^{-}$ mediated cAMP production (Willard et al., 2012b; Wootten et al., 2013b). A weak potentiation of compound 2-mediated cAMP production (or surrogate readouts such as cAMP response element-driven luciferase reporter) has also been reported for interaction of this ligand with truncated forms of exendin, including exendin ${ }_{5-39}$, exendin $_{7-39}$, and exendin ${ }_{9-39}$, even in the absence of any measurable intrinsic activity (Coopman et al., 2010; Cheong et al., 2012).

Intriguingly, if the cooperative effect of BETP or compound 2 is studied over a broader array of signaling endpoints, then marked differences in effect are observed (Koole et al., 2012a; Li et al., 2012a; Wootten et al., 2012, $2013 \mathrm{~b})$. In contrast to the selective augmentation of cAMP response for oxyntomodulin, the allosteric ligands confer a similar, modest enhancement of arrestin recruitment for both GLP-1 and oxyntomodulin (Willard et al., 2012b; Wootten et al., 2013b), although a greater 
degree of negative cooperativity is seen on pERK responses of GLP-1 $1_{7-36}$ amide versus oxyntomodulin. Thus, the allosteric ligands also alter the signaling bias mediated by endogenous peptides. This is perhaps not surprising as both signaling bias and the cooperative effects of cobound ligands are driven through changes to the ensemble of conformations that the receptor samples (Wootten et al., 2013a). Indeed, the allosteric ligandbound receptor can effectively be considered a distinct receptor for the interaction of the natural ligand(s).

Remarkably, both BETP and compound 2 very strikingly enhanced the cAMP response of the principal GLP-1 metabolite, GLP-1 $1_{9-36}$ amide, by up to $\sim 400$-fold in the case of compound 2 ( $\mathrm{Li}$ et al., 2012a; Wootten et al., 2012). Parallel augmentation of insulin secretion was observed in isolated rat islets and in vivo when pharmacological levels of the metabolite were coadministered with subthreshold levels of BETP (Wootten et al., 2012). The augmentation of signaling was principally limited to cAMP production, although weak potentiation of $\mathrm{pERK}$ and $\mathrm{iCa}^{2+}$ signaling was seen in HEK293 cells recombinantly expressing the receptor and INS-1E cells (Li et al., 2012a), but not in CHO cells recombinantly expressing the receptor (Wootten et al., 2012), providing further evidence that allosteric modulators can alter the signal bias of the receptor. Nonetheless, the lack of effect on the extended GLP-1 peptide indicates that the magnitude of the cooperative effect of BETP and compound 2 is not driven solely by the intrinsic efficacy of the activating peptide.

Ligands such as BETP and compound 2 are highly electrophilic and can form adducts with free cysteine residues (Eng et al., 2013). This is a feature of the action of these molecules to form, in particular, the covalent interaction with $\mathrm{C} 347^{6.36 \mathrm{~b}}$ in ICL3/TM6 that is critical for both the intrinsic efficacy and the cooperative allosteric effect (Nolte et al., 2014). Nevertheless, the covalent modification of the cysteine is insufficient to explain the full extent of activity of these compounds as BETP and compound 2 have differences in their profile of intrinsic efficacy (see above) and also in their cooperative effect (Wootten et al., 2013b). Interestingly, there is a naturally occurring polymorphism that can appear at position 333 of the human receptor, being either a serine or a cysteine with the latter occurring only rarely (Koole et al., 2011, 2015). However, the C333 ${ }^{\text {ICL3 }}$ variant selectively abrogates compound 2mediated cAMP production and cooperativity between compound 2 and oxyntomodulin for this pathway (Koole et al., 2011) while maintaining peptide-mediated signaling, suggesting that the environment surrounding $\mathrm{C} 347^{6.36 \mathrm{~b}}$ is important for the action of compound 2 . In contrast to the effect of the C333 ${ }^{\text {ICL3 }}$ polymorphism, the M149 ${ }^{1.44 b}$ polymorphic variant has no effect on the intrinsic efficacy of compound 2 , but, as noted above, causes a very marked attenuation of peptide-mediated cAMP signaling with over 100 -fold loss of GLP- $1_{7-36}$ amide or exendin potency. At this mutant, compound 2 could restore the potency of both GLP-1 $1_{7-36}$ amide and exendin to that of the wild-type receptor (Koole et al., 2011). Thus, allosteric modulators have the capacity to alleviate genetic disease arising from the loss of function of GLP-1R.

Disruption of the TM4 dimer interface of GLP-1R also abolishes the intrinsic efficacy of BETP and compound 2 , but the cooperative augmentation of cAMP signaling by oxyntomodulin is maintained, indicating that the allosteric effect occurs in cis at least for this class of ligands (Harikumar et al., 2012).

Although compounds such as BETP and compound 2 were among the first recognized and the most widely studied allosteric modulators of GLP-1R, a number of additional compounds have been reported to act as modulators of peptide response. This includes the flavonol, quercetin, which lacks intrinsic activity, but could selectively augment calcium signaling of efficacious peptides such as GLP-1 $1_{7-36}$ amide, GLP- $1_{7-37}$, and exendin, albeit that an inhibition of response was seen with high concentrations of quercetin (Koole et al., 2010; Wootten et al., 2011). This augmentation of $\mathrm{iCa}^{2+}$ was dependent upon the presence of a 3-hydroxyl group on the flavone backbone and was improved when a $3^{\prime} 4^{\prime}$ dihydroxyl modification was present (Wootten et al., 2011). An additional series of compounds that could selectively enhance $\mathrm{iCa}^{2+}$ signaling was recently reported following high-throughput screening (Morris et al., 2014). Unlike the flavonols, this series of compounds had significant intrinsic efficacy for mobilization of $\mathrm{iCa}^{2+}$. An exemplar from this series, termed (S)-9b, was examined in further detail, in which it was reported to also augment liraglutide-mediated GLP-1R internalization in recombinant cells and exendin-mediated insulin secretion in primary mouse islets (Morris et al., 2014). This latter effect occurred in both high and low glucose, which would be problematic from a therapeutic standpoint, but may also require further investigation to determine whether this is mediated by GLP-1R. Likewise, compound (S)-9b reduced haloperidol-induced catalepsy in rats (Morris et al., 2014), but, again, it needs to be confirmed whether this effect is mediated via GLP-1R. In other work, using virtual screening against a molecular model of GCGR, de Graaf et al. (2011) identified a number of compounds that had activity at both GCGR and GLP-1R, including weak antagonists and one compound that acted as an inhibitor of glucagon-mediated cAMP production at GCGR, but as a weak positive modulator of GLP-1R-mediated cAMP response.

At this point in time, the physiologic or therapeutic implications of biased signaling are largely unknown. Despite differences in the signaling/regulatory profile of compounds such as Boc5 or TT15, these compounds (or similar analogs) have demonstrated efficacy in modulation of ex vivo and in vivo insulin secretion (Chen et al., 2007; He et al., 2012). Allosteric compounds such 
as BETP or compound 2 likewise can augment insulin secretion, albeit that this is complicated by endogenous circulating peptides. Pharmacologically, both BETP and compound 2 can augment responses of oxyntomodulin and the GLP-1 metabolite (GLP-19-36 amide), and this is linked to increased in vivo insulin secretion, at least in the context of threshold doses of the allosteric and orthosteric peptides (Willard et al., 2012b; Wootten et al., 2012). In vitro assessment of the effect of the modulators on the signaling profile of oxyntomodulin or GLP-19-36 amide indicates that their most prominent effect is to augment cAMP production, suggesting that this alone may be sufficient to improve insulin secretion, at least in the context of the baseline signaling of the peptides/modulators. The significance for GLP-1R function outside of insulin secretion is even less clear, as there has been very limited assessment of these other functions and none controlled for the influence of biased signaling. A major current limitation is the range of tools available to probe the significance of signaling differences. Most nonpeptidic compounds have low potency, nonfavorable pharmacokinetic profiles, or unknown interaction with other targets that limits their utility. Exendin is biased relative to GLP-1 $1_{7-36}$ amide, but this difference is subtle in the context of canonical signaling, making interpretation of physiologic data difficult. Oxyntomodulin is the most biased of the characterized peptides, displays good affinity for GLP-1R, and has distinctions in its physiologic actions from GLP-1, but dissecting the importance of this bias is complicated by its significant interaction with GCGR (Pocai, 2013). Nonetheless, this may be a useful tool to study biased signaling in GCGR knockout animals. Additional approaches to infer the potential role of selective activation of signaling/regulatory pathways include the generation of knock-in mice that have receptors designed to selectively abrogate signaling via a specific pathway (e.g., G protein versus arrestin), although the development of potent biased peptide ligands could also provide novel scope for better understanding this phenomenon.

\section{Receptor Regulation}

1. Receptor Desensitization. The activity of GLP-1R, like all GPCRs, is regulated by a coordinated balance between molecular mechanisms governing receptor signaling, desensitization, and resensitization. Receptor desensitization, or the reduced responsiveness of GPCR signaling to an agonist with time, is an important physiologic feedback mechanism that protects against both acute and chronic receptor overstimulation (Ferguson, 2001). This has important biologic and therapeutic implications.

GLP-1R undergoes rapid and reversible homologous and heterologous desensitization that has been observed in recombinant cell systems, in primary islets of Langerhans, and in insulinoma $\beta$ cell lines (Fehmann and Habener, 1991; Gromada et al., 1996; Widmann et al., 1996a,b). Homologous desensitization refers to the loss of response to subsequent agonist stimulation following direct stimulation of the receptor and can occur through controlling the number of receptors present at the cell surface or by regulating the efficacy of the receptors at the cell surface. Heterologous desensitization describes receptor activation-independent regulation of receptors as well as mechanisms that occur after receptor activation that do not discriminate between activated, and nonactivated, receptors (Ferguson, 2001).

Acute incubation of islet cells with native GLP-1 induces rapid homologous GLP-1R desensitization in vitro (Fehmann and Habener, 1991; Gromada et al., 1996). In addition, exendin- 4 also produces GLP-1R desensitization in islet cells following both acute and chronic exposure; however, this ligand is associated with a greater degree of desensitization compared with comparable incubations with GLP-1 (Baggio et al., 2004). Heterologous desensitization has been demonstrated in islets in response to multiple stimuli. For instance, GLP-1R heterologous desensitization was observed via acute exposure to phorbol myristate acetate (PMA), an activator of PKC (Widmann et al., 1996a,b; Baggio et al., 2004). In addition, prolonged hyperglycemia can induce desensitization, followed by downregulation with diminished responses to GLP-1R agonists that include weakened insulin secretion, reduced phosphorylation of CREB, impaired cAMP responses and PKA activity, and a reduction in GLP-1R mRNA (Rajan et al., 2015).

There is also the possibility that heterologous desensitization may occur due to activation of other receptors expressed in $\beta$ cells that are involved in tightly controlling insulin secretion. To date, this has only been assessed with the ligand for GIPR. GLP-1R can heterodimerize with GIPR in vitro, with evidence that this heterodimerization can alter cellular signaling and trafficking profiles of GLP-1R (Schelshorn et al., 2012; Roed et al., 2015). However, despite their overlapping functions for signaling and insulin secretion, GIP does not produce meaningful heterologous desensitization of GLP-1R in islet cell studies (Rajan et al., 2015).

2. Underlying Mechanisms. Desensitization is a consequence of a number of different mechanisms. They include uncoupling the receptor from heterotrimeric $\mathrm{G}$ proteins in response to receptor phosphorylation, the internalization of receptors to intramembranous compartments, and the downregulation of the total complement of receptors (Ferguson, 2001).

a. GLP-1R phosphorylation. The most rapid mechanism by which GPCRs are desensitized is through the covalent modification of the receptor as a consequence of phosphorylation by intracellular kinases, either G proteincoupled receptor kinases (GRKs) or second messenger kinases (Ferguson, 2001). Both homologous and heterologous desensitization of GLP-1R are accompanied by phosphorylation of serine residues within the last 33 amino acids of the C-terminal tail (Widmann et al., 1996a,b). 
b. GRKs. For most GPCRs, homologous desensitization is thought to involve phosphorylation by GRKs that results in recruitment of $\beta$-arrestins. In vitro studies in fibroblast cells expressing GLP-1R revealed that homologous desensitization was associated with phosphorylation of serine residues 441/442, 444/445, and 451/452 in the $\mathrm{C}$ terminus (Widmann et al., 1997). Desensitization of GLP-1R-mediated cAMP responses after a first initial exposure of cells to GLP-1 was strictly dependent on the extent of phosphorylation with no, intermediate, or maximum phosphorylation observed in the presence of one, two, or three of the serine doublet phosphorylation sites, respectively.

Although various groups, using a number of techniques, have demonstrated that stimulation of GLP-1R leads to recruitment of both GRKs and $\beta$-arrestins (Jorgensen et al., 2005, 2011; Wootten et al., 2013a), to date, the direct involvement of these proteins in desensitization of GLP-1R response has not been demonstrated. In recombinant systems, GRK2 can interact with GLP-1R in response to stimulation by GLP-1 (Jorgensen et al., 2007, 2011). In addition, GRK2 has been linked to potentiation of $\beta$-arrestin 2 recruitment to the receptor. $\beta$-arrestin 1 is also recruited to GLP-1R upon activation by agonist ligands (Sonoda et al., 2008; Wootten et al., 2013a); however, whether recruitment of these proteins is required for internalization is currently not clear.

c. Second messenger protein kinases. Heterologous desensitization of GLP-1R occurs at least in part via the second messenger kinases, cAMP-dependent PKA and PKC (Widmann et al., 1996b; Rajan et al., 2015). Direct activation of PKC by PMA markedly reduced the amplitude of GLP-1R-mediated calcium responses, whereas inhibitors of PKC slowed desensitization and increased the duration of calcium transients (Gromada et al., 1996). Using fusion proteins of wild-type and mutant GLP-1R C-terminal tails, in vitro phosphorylation experiments revealed that PKC-mediated phosphorylation occurred predominantly at residues $431 / 432$, and, whereas positions $444 / 445$ and $451 / 452$ could be phosphorylated by PKC, they were poor substrates. In these studies, the serine doublet $441 / 442$ was not a substrate for PKC (Widmann et al., 1996a). In contrast, studies performed in intact COS-7 cells showed all four of these doublets could be phosphorylated following PKC activation by PMA. This could be attributed to different isoforms of PKC in intact cells or the possibility that other kinases downstream of PKC activation may be important for PMA-induced GLP-1R phosphorylation. Phosphorylation of at least two of these serine doublets was required to engender PMA-induced heterologous desensitization, with removal of any pair of doublets leading to receptors that were completely resistant to PMA-induced GLP-1R desensitization (Widmann et al., 1996a).

PKA has been implicated as a mediator of desensitization and downregulation of GLP-1R from the cell surface in pancreatic islets in conditions of chronic hyperglycemia (Rajan et al., 2015). In MIN6 cells, reductions in cell surface receptor numbers in conditions of high glucose were mimicked by overexpression of a constitutively active PKA or continuous activation by forskolin. In addition, inhibition of PKA activity attenuated glucose-mediated downregulation of GLP-1R from the cell surface of these cells. Phosphorylation at serine 301 , within the third intracellular loop of the receptor, is implicated in this PKA-mediated activity, with mutation of this residue abolishing glucose-dependent loss of the receptor from the cell surface. This was associated with a loss of an interaction between the receptor and the small ubiquitin-related modifier, SUMO (Rajan et al., 2015). Sumoylation of GLP-1R causes intracellular retention of the receptor and desensitization of receptor signaling as well as prevents resensitization of the receptor back to the cell surface (Rajan et al., 2012). This heterologous desensitization and subsequent downregulation of GLP-1R on the $\beta$ cell surface by chronic hyperglycemic conditions have substantial implications for the efficacy of GLP-1-based therapies. Because SUMO expression is increased in mouse islets exposed to high glucose (Rajan et al., 2012), its interaction with GLP-1R may in part contribute to the reduced efficacy of incretin therapies in some T2DM patients with poorly controlled hyperglycemia (Fritsche et al., 2000; Stumvoll et al., 2002; Kjems et al., 2003).

3. In Vivo Evidence of Desensitization. Given the clinical interest in the therapeutic benefits of achieving sustained chronic elevations of GLP-1R agonists in the plasma through repeated or continual administration, the scope of GLP-1R desensitization has direct therapeutic relevance. Up to now, only a few studies have addressed the relevance of GLP-1R desensitization in vivo. Chronic or intermittent intracerebroventricular GLP-1 administration inhibited food intake and reduced weight gain in rats (Davis et al., 1998). Similarly, chronic intermittent administration of GLP-1R agonists to diabetic rodents is associated with inhibition of food intake, improvement of glycemia, and reduction in hemoglobin A1c (HbA1c), demonstrating that repeated administration of GLP-1 is not associated with a diminished therapeutic response in vivo (Szayna et al., 2000; Rolin et al., 2002; Kim et al., 2003).

Considering the prolonged activity and stability of GLP-1 mimetics compared with native GLP-1 (Young et al., 1999), it seems likely that islet cells and extrapancreatic GLP-1R would be exposed for a greater period of time with these ligands than the endogenous peptides. In vitro studies showed that exendin- 4 is more potent than GLP-1 in producing GLP-1R desensitization; however, chronic exposure to exendin-4 in normal or transgenic mice that express exendin-4 was not associated with significant downregulation of GLP1R-dependent responses coupled to glucose homeostasis (Baggio et al., 2004). Furthermore, patients treated 
with twice-daily exendin-4 or once-weekly liraglutide continue to exhibit a decrease in $\mathrm{HbA1c}$ and marked reductions in postprandial glycemic excursion (Buse et al., 2004; de Wit et al., 2014). Therefore, although in vitro experiments clearly demonstrate that GLP-1R has the capacity for desensitization, there is little evidence that it undergoes clinically meaningful desensitization in vivo in terms of glucose regulation.

There is, however, some limited evidence for desensitization occurring in vivo (Nauck et al., 2011). Administering native GLP-1 continuously into healthy human subjects for 8.5 hours, followed by assessment of glucoregulatory responses to liquid test meals given 5 hours apart with ongoing continuous GLP-1 infusion, demonstrated a reduced ability of GLP-1 to suppress gastric emptying and glucagon levels by the second test meal. In addition, levels of pancreatic polypeptide, a marker of vagal activation, were not as inhibited during the second test meal compared with the first. However, C-peptide and insulin levels were preserved with only a small reduction in the second meal. These studies reveal that even short-term continuous GLP-1R stimulation may be associated with some degree of rapid tachyphylaxis, most evident in effects mediated through the vagus nerve and gastric emptying (Nauck et al., 2011). Despite this evidence for in vivo desensitization, the physiologic significance of this is still unclear.

4. Receptor Internalization. GLP-1R rapidly internalizes as a complex associated with its bound ligand. This has been observed for GLP-1, exendin-4, liraglutide, and compound 2 (Widmann et al., 1995; Kuna et al., 2013; Roed et al., 2014). In BRIN-BD11 cells, antibodylabeled GLP-1R and fluorescently labeled GLP-1 were colocalized at the perinuclear space following internalization (Kuna et al., 2013). Furthermore, internalization of fluorescently labeled GLP-1, exendin-4, and liraglutide has been observed in both recombinant cell systems and primary mouse pancreatic islets (Roed et al., 2014). There is also some evidence of ligand-directed bias with GLP-1R internalization, although this has not been directly quantified. The potencies for internalization by GLP-1 and exendin-4 were 10-fold higher than liraglutide, although kinetics of internalization were similar for all three ligands (Roed et al., 2014).

Currently, the mechanism for GLP-1R internalization in vivo is unclear, as there is evidence that this mechanism may be cell-type dependent (Widmann et al., 1995; Vazquez et al., 2005; Syme et al., 2006; Thompson and Kanamarlapudi, 2015). As GLP-1R is expressed in multiple tissues throughout the body with distinct physiologic functions depending upon the location of the receptor, this implies that the mechanism and role of receptor internalization and desensitization may be tissue-dependent. In different cell backgrounds, two distinct mechanisms of internalization have been observed, with both mechanisms dependent on dynamin (Widmann et al., 1995; Syme et al., 2006; Kuna et al., 2013).
Studies performed in CHO and CHL cells recombinantly expressing GLP-1R revealed internalization via clathrin-coated pits, although these studies indicated that there might not be complete internalization via this method (Widmann et al., 1995; Vazquez et al., 2005). Studies in CHL fibroblasts demonstrated that the same three phosphorylation sites linked to homologous desensitization of GLP-1R-mediated cAMP responses (441/442, 444/445, and 451/452) are also important for internalization, supporting a correlation between phosphorylation at these sites and internalization (Widmann et al., 1997). However, the exact contribution of each phosphorylation site to desensitization of the cAMP response and internalization is different, indicating that the precise molecular basis for control of receptor desensitization and endocytosis is, at least in part, distinct. In addition to phosphorylation of these serine doublets, a region of the C-terminal tail close to the bottom of TMD7 is also of importance in mediating agonist-dependent GLP-1R internalization (Vazquez et al., 2005).

Traditionally for GPCRs, $\beta$-arrestin recruitment targets receptors for clathrin-mediated internalization (Ferguson, 2001). However, the literature indicates that this may not be the case for GLP-1R in some cell backgrounds, despite the ability of the receptor to interact with these proteins (Syme et al., 2006). Although there is little literature on the role that $\beta$-arrestin 2 plays in GLP$1 \mathrm{R}$ internalization and desensitization, there is some evidence that these processes can occur independently of $\beta$-arrestin recruitment, in a clathrin-independent mechanism. Studies performed in HEK293, COS-7, and MIN6 cells exhibited caveolin-1 regulation of internalization (Syme et al., 2006; Thompson and Kanamarlapudi, 2015). GLP-1R contains a classic caveolin-1-binding motif within the second intracellular loop such that it interacts and colocalizes with the protein intracellularly. Occurrence of this interaction was also supported by studies using inhibitors of caveolin-1. This mechanism of GLP$1 \mathrm{R}$ internalization has been proposed to occur through activation of $\mathrm{G} \alpha \mathrm{q}$ proteins, followed by activation of PKC (Thompson and Kanamarlapudi, 2015). In support of $\beta$-arrestin-independent internalization, it was shown that knockdown of $\beta$-arrestin 1 in INS- 1 cells had no effect on internalization or desensitization of GLP-1R (Sonoda et al., 2008).

Although internalization can contribute to desensitization of receptors, internalization and desensitization are two distinct phenomena. Internalization has the potential to contribute to termination of some GLP-1Rmediated signaling events; however, it does not terminate all components of this signaling (Kuna et al., 2013; Roed et al., 2015). A recent study revealed that inhibition of internalization using a dominant-negative form of dynamin (K44E) resulted in decreased cAMP formation, ERK1/2 phosphorylation, and calcium signaling, suggesting that at least some of the signaling mediated 
by these pathways occurs via internalized receptors. In addition, inhibition of GLP-1R internalization also attenuates insulin secretion, confirming that internalization is an integral part of the signaling process for this receptor (Kuna et al., 2013). Using fluorescently labeled ligands and receptors, studies in recombinant systems displayed colocalization of internalized GLP$1 \mathrm{R}$ with adenylate cyclase in endosomes (Kuna et al., 2013). Taken together, these findings all imply that GLP-1R continues to signal following internalization, and that internalization is not necessarily part of the mechanism for desensitization. This level of spatiotemporal control achieved by the compartmentalization of signaling through internalization may be vital to specific, fine-tuned responses from GLP-1R activation and may have substantial implications both physiologically and therapeutically for targeting this receptor in disease management.

5. Receptor Resensitization. GLP-1R resensitization has not been extensively studied. Following desensitization, GLP-1R-mediated calcium signaling resensitizes after removal of extracellular ligand within 1 hour and does not require de novo protein synthesis (Gromada et al., 1996). After internalization, GLP-1R colocalizes with transferrin, a marker for recycling endosomes, suggesting that it is recycling GLP-1R that is returned back to the cell surface after internalization (Roed et al., 2014). Moreover, there is evidence in HEK293 cells that GLP-1R recycles back to the cell surface in response to GLP-1, exendin-4, and liraglutide, even after prolonged agonist treatment. However, recycling was two to three times slower for exendin- 4 and liraglutide compared with GLP-1 (Roed et al., 2014). In the presence of GLP-1, receptors were found in transferrin-positive endosomes up to 45 minutes post ligand addition, whereas in the presence of exendin- 4 and liraglutide colocalization of internalized GLP-1R and transferrin was observed for up to 60 minutes following ligand addition. Resensitization is important, as irreversible desensitization would leave a cell unable to respond to external stimuli, and therefore this mechanism protects against prolonged desensitization (Ferguson, 2001). Nonetheless, to date, the mechanism of GLP-1R resensitization remains largely unknown and requires further investigation.

There is also evidence of partial degradation of GLP$1 \mathrm{R}$ upon prolonged activation with exendin-4, liraglutide, and GLP-1, with colocalization observed between the receptor and lysosomes (Kuna et al., 2013; Roed et al., 2014). This mechanism leads to downregulation of receptor numbers.

\section{Pharmacological Tools}

\section{A. Traditional Bioassays}

As GLP-1 exerts its vital physiologic and pharmacological functions through interacting with its receptor on the cell membrane, it is important to measure and determine the activities of the peptide and its analogs through the biologic actions of the protein. There are traditional bioassays (Table 2) to determine receptorbinding affinity and activation of the receptor, and several unique methods have been reported for assessing molecular recognition and biochemical consequences. In addition, molecular imaging of the receptor has demonstrated a potential for noninvasive monitoring of pancreatic $\beta$ cells, which may be of great value in the diagnosis and prognosis of diabetes, tracking the disease progression, and evaluating therapeutic interventions, including islet regeneration and transplantation.

\section{B. Other Bioassays}

1. Receptor Trafficking. Endocytosis of GPCRs and postendocytic trafficking between recycling and endosomal degradation are fundamental mechanisms that control the signaling capacity of receptors. Syme et al. (2006) studied the trafficking of green fluorescent protein-fused GLP-1R stably expressed on HEK293 cells in response to a GLP-1 agonist. It was found that GLP-1R associates with caveolin-1 in the lipid rafts of the cell membrane of HEK293 cells transfected with the green fluorescent protein-GLP-1R plasmid and MIN6 cells endogenously expressing the GLP-1 receptor. With confocal fluorescence microscopy and immunoprecipitation techniques, it was observed that stimulation by an agonist induces rapid and extensive internalization of the receptor in association with caveolin-1, and nearly all of the receptor was internalized after 15 minutes.

Similarly, Roed et al. (2014) combined microscopy and a time-resolved fluorescence resonance energy transfer-based technique for monitoring both receptor trafficking and signaling in living cells. The GLP-1 receptor rapidly and repeatedly internalizes and recycles upon ligand activation. GLP-1R recycling was found to be two to three times slower when induced by exendin-4 and liraglutide, compared with GLP-1 at equipotent concentrations. Independent of the ligand, activated receptors demonstrated cycling for a prolonged period of time as well as sustained cAMP signaling.

2. Immunoassays. Determination of incretins, especially endogenous ones, by immunologic assays is considered difficult because differential processing of precursor molecules gives rise to a number of different peptides that may crossreact with antisera raised against GLP-1 (Deacon and Holst, 2009). Subsequent degradation of the peptides by DPP-4 and NEP 24.11 further complicates the assay process (Heijboer et al., 2011).

When the full processing pattern of proglucagon was not fully elucidated, the assays were directed to the middle region of GLP-1 and became independent to the processing pattern. This caused peptides with a deletion or extension at either the $\mathrm{N}$ or $\mathrm{C}$ terminus to be detected in addition to GLP-1 itself. Antisera were then made toward either the $\mathrm{C}$ terminus (Hendrick et al., 1993; Holst et al., 1994; Ørskov et al., 1994) or the $\mathrm{N}$ terminus 
(Gutniak et al., 1996). However, it was found that C-terminally directed antisera reacted with peptides truncated or extended at the $\mathrm{N}$ terminus and could not distinguish the full-length GLP-1 from the metabolite resulting from DPP-4 degradation. Similarly, N-terminally directed antisera could not differentiate the intact peptide from truncated or extended peptides at the $\mathrm{C}$ terminus.

Kirk and coworkers developed a sandwich enzymelinked immunosorbent assay for determining exogenous GLP-1 $1_{7-36}$ amide in plasma samples collected from pharmacokinetic studies (Pridal et al., 1995). It is referred to as active GLP-1 assay. This experiment employs an N-terminally directed antibody (polyclonal rabbit antibody directed to the residues at position 714) and a C-terminally directed antibody (monoclonal mouse antibody directed to the residues at position 26 33 ). When compared with a radioimmunoassay employing a polyclonal rabbit antibody directed to an epitope in the midregion of the peptide, it was found to be superior due to reduced crossreactivity with GLP-1 fragments. It has a working range from 10 to $500 \mathrm{pmol} / \mathrm{L}$.

3. Pancreatic $\beta$ Cell Regeneration. Besides insulin secretion from the pancreatic $\beta$ cells in a glucosedependent manner, GLP-1 can induce regeneration of the cells evidenced by immunohistochemical staining studies (Edvell and Lindstrom, 1999; Xu et al., 2006). PDX-1 is essential for pancreogenesis, pancreatic cell differentiation, and maturation. Perfetti and coworkers demonstrated that continuous infusion of GLP-1 to young and old rats upregulates PDX-1 expression in islets and total pancreas (Hui and Perfetti, 2002). They also found induced pancreatic cell proliferation and $\beta$ cell neogenesis (Perfetti et al., 2000).

\section{Molecular Imaging}

Pancreatic $\beta$ cells play an important role in glucose homeostasis, and their destruction is well documented with diabetes, in particular type 1 . Whereas several clinical parameters, such as plasma glucose, glycated $\mathrm{HbA1c}$, insulin, and C-peptide levels, indicate onset and progress of the disease, the majority of the $\beta$ cells are destroyed by the time the symptoms appear (Matveyenko and Butler, 2008). Late diagnosis of the disease results from the remaining $\beta$ cells compensating for the loss of insulin production due to cell death, and abnormalities in blood glucose concentrations are not typically observed until $\beta$ cell mass (BCM) is diminished by more than $50 \%$ (Souza et al., 2006). Thus, noninvasive imaging of BCM would provide accurate and in-time status as well as useful prediction. $\beta$ cell imaging has potential in monitoring the disease progression, evaluating efficacy of therapeutic interventions in preserving and restoring BCM, and following up $\beta$ cell replacement therapies such as islet transplantation (Halban, 2004).

Imaging modalities, such as positron emission tomography (PET), magnetic resonance imaging, as well as other nuclear imaging techniques like single-photon emission-computed tomography (SPECT), and optical absorption or fluorescence spectroscopy and imaging, have shown promises in visualizing and quantitating molecular targets (Souza et al., 2006). However, assessing $\mathrm{BCM}$ in vivo has been challenging because the $\beta$ cells that exist in pancreatic islets are small (50-300 $\mu \mathrm{m}$ in diameter), scarce (1-2\% of pancreatic mass), and scattered throughout the organ (Ueberberg et al., 2009).

As nonselective imaging modalities like magnetic resonance imaging and computed tomography have failed to offer a reliable detection method, $\beta$ cell-specific biomarkers have been investigated and evaluated (Shiue et al., 2004; Souza et al., 2006; Saudek et al., 2008; Malaisse et al., 2009; Moore, 2009; Ichise and Harris, 2010). Although a number of potential candidates for $\beta$ cell imaging have been reported, such as vesicular monoamine transporter, GLP-1R, SUR1, glucose transporter 2, glycogen, zinc transporters, fluorodithizone, and monoclonal antibodies, many did not show promising results due to either low expression levels or insufficient $\beta$ cell specificity (Murthy et al., 2008; Schneider, 2008; Moore, 2009). Vesicular monoamine transporter is expressed at dopamine nerve ends in the CNS and pancreatic $\beta$ cells, and its ligands, ${ }^{11} \mathrm{C}$ - and ${ }^{18} \mathrm{~F}$-labeled dihydrotetrabenazine derivatives, have demonstrated a potential for $\beta$ cell imaging (Souza et al., 2006; Harris et al., 2008; Kung et al., 2008). However, controversial findings of nonspecific binding of $\left[{ }^{11} \mathrm{C}\right]$-dihydrotetrabenazine in human pancreas raised questions for its clinical application (Goland et al., 2009).

Compared with other $\beta$ cell biomarkers, GLP-1R showed promise because of its highly specific expression in $\beta$ cells and strong interaction with its ligands (Tornehave et al., 2008). However, its endogenous ligand, GLP-1, is difficult to be used as an imaging agent because of its extremely short half-life (II. Glucagon-Like Peptide-1) resulting from rapid metabolic degradation. This emphasizes that $\beta$ cell imaging via GLP-1R as a biomarker requires GLP-1 analogs that have high metabolic stability as well as strong binding affinity.

Exendin-3 and exendin- 4 are found to be resistant to DPP-4 degradation, and these stable GLP-1R agonists have been developed for $\beta$ cell imaging (Gotthardt et al., 2006; Wild et al., 2006, 2010; Brom et al., 2010; Reiner et al., 2010; Wu et al., 2011; Connolly et al., 2012). Alternatively, bicyclic GLP-1 analogs developed by Ahn and coworkers have also demonstrated outstanding results in determining BCM (Ahn et al., 2011; Gao et al., 2011). A metal chelator, such as DOTA, NOTA, or DTPA, was conjugated to these peptides and subsequently labeled with a radiotracer (e.g., ${ }^{64} \mathrm{Cu},{ }^{68} \mathrm{Ga},{ }^{99 \mathrm{~m}} \mathrm{Tc}$, and $\left.{ }^{111} \mathrm{In}\right)$ for PET- and SPECT-imaging experiments.

A SPECT probe was developed based on exendin-4, $\left[\mathrm{Lys}^{40}\left(\mathrm{Ahx}-\mathrm{DTPA}-{ }^{111} \mathrm{In}\right) \mathrm{NH}_{2}\right.$ ]-exendin-4, to target GLP1R for imaging insulinoma in Rip1Tag2 transgenic mice and showed high tumor uptake (Wild et al., 2006; Wicki et al., 2007). Although imaging agents derived from 
TABLE 2

Summary of traditional biologic assays for GLP-1 analogs

\begin{tabular}{|c|c|c|}
\hline Molecular/Cellular Measurement & Description/Outcome & References \\
\hline \multicolumn{3}{|l|}{ Receptor-binding assays: } \\
\hline Radioligand competition & $\begin{array}{l}\text { Determines binding affinity }\left(\mathrm{IC}_{50} \text { or } \mathrm{K}_{\mathrm{d}}\right) \text { by } \\
\text { measuring radioactivity remaining on the } \\
\text { receptor after competitive inhibition of } \\
\text { radioligand with a GLP-1 analog. }\end{array}$ & $\begin{array}{l}\text { Mathi et al., 1997; Tibaduiza et al., 2001; Xiao } \\
\text { et al., } 2001\end{array}$ \\
\hline $\begin{array}{l}\text { Time-resolved fluorescence resonance } \\
\text { energy transfer (FRET) }\end{array}$ & $\begin{array}{l}\text { Determines binding affinity }\left(\mathrm{IC}_{50}\right) \text { by measuring } \\
\text { a decrease in FRET signal between Tb-labeled } \\
\text { receptor and fluorescent ligand after } \\
\text { competitive inhibition with a GLP-1 analog. }\end{array}$ & $\begin{array}{l}\text { Maurel et al., 2008; Zwier et al., 2010; Roed } \\
\text { et al., } 2014\end{array}$ \\
\hline $\begin{array}{l}\text { Circular dichroism and fluorescence } \\
\text { spectroscopies }\end{array}$ & $\begin{array}{l}\text { Determines binding affinity by measuring } \\
\text { conformational changes of a receptor protein } \\
\text { upon ligand binding. }\end{array}$ & Runge et al., 2007 \\
\hline Isothermal titration calorimetry & $\begin{array}{l}\text { Determines thermodynamic parameters of } \\
\text { binding, such as the dissociation constant, } \\
\text { enthalpy change, entropy change, and reaction } \\
\text { stoichiometry by measuring heat changes } \\
\text { during receptor-ligand interaction. }\end{array}$ & $\begin{array}{l}\text { Wiseman et al., 1989; Bazarsuren et al., 2002; } \\
\text { Donnelly, } 2012\end{array}$ \\
\hline $\begin{array}{l}\text { Total-internal reflection fluorescence } \\
\text { imaging }\end{array}$ & $\begin{array}{l}\text { Determines equilibrium constants and } \\
\text { dissociation rates by monitoring fluorescence- } \\
\text { labeled single molecule on lipid bilayer. }\end{array}$ & Fox et al., 2009; Myers et al., 2012 \\
\hline Surface plasmon resonance & $\begin{array}{l}\text { Determines dissociation constants by real-time } \\
\text { measurement of receptor-ligand interaction. }\end{array}$ & $\begin{array}{l}\text { Bazarsuren et al., 2002; Schroder-Tittmann } \\
\text { et al., 2010; Locatelli-Hoops et al., } 2013\end{array}$ \\
\hline Photoaffinity labeling & $\begin{array}{l}\text { Identifies residues of peptide and receptor at } \\
\text { binding interface that are spatially proximal } \\
\text { to each other. }\end{array}$ & $\begin{array}{l}\text { Chowdhry and Westheimer, 1979; Ji et al., 1997; } \\
\text { Vodovozova, 2007; Chen et al., 2009; Miller } \\
\text { et al., } 2011\end{array}$ \\
\hline \multicolumn{3}{|l|}{ Receptor functional assays: } \\
\hline $\begin{array}{l}\text { Homogeneous time-resolved fluorescence } \\
\text { (HTRF) or alpha screen cAMP assays }\end{array}$ & $\begin{array}{l}\text { HTRF technology is an immunoassay based on a } \\
\text { FRET between a Tris-bipyridine europium } \\
\text { cryptate used as a long-lived fluorescent donor } \\
\text { and a chemically modified allophycocyanin } \\
\text { used as acceptor. Alpha technology is a bead- } \\
\text { based proximity assay. }\end{array}$ & $\begin{array}{l}\text { Gesellchen et al., 2006; Einhorn and } \\
\text { Krapfenbauer, } 2015\end{array}$ \\
\hline Radioimmunoassay & $\begin{array}{l}\text { Determines receptor-stimulating potency }\left(\mathrm{EC}_{50}\right) \\
\text { by quantitative analysis of cAMP production } \\
\text { with immobilized anti-cAMP or anti-cGMP } \\
\text { antibodies and radiolabeled cAMP/cGMP. }\end{array}$ & Farmer et al., 1975; Wheeler et al., 1995 \\
\hline FRET-based cAMP assay & $\begin{array}{l}\text { Determines receptor-stimulating potency }\left(\mathrm{EC}_{50}\right) \\
\text { by measuring a decreased FRET signal } \\
\text { between fluorescent proteins (CFP and YFP) } \\
\text { and Epac protein. }\end{array}$ & $\begin{array}{l}\text { Holz, 2004; Nikolaev et al., 2004; Landa et al., } \\
\text { 2005; Harbeck et al., 2006; Sloop et al., } 2010\end{array}$ \\
\hline Luciferase reporter assay & $\begin{array}{l}\text { Determines receptor-stimulating potency }\left(\mathrm{EC}_{50}\right) \\
\text { by measuring luminescence that is increased } \\
\text { by transcription of transfected luciferase } \\
\text { reporter plasmid linked to cAMP response } \\
\text { element. }\end{array}$ & $\begin{array}{l}\text { Grynkiewicz et al., 1985; Cullinan et al., 1994; } \\
\text { Bode et al., 1999; Miranda et al., 2008; Murage } \\
\text { et al., 2008; Smale, } 2010\end{array}$ \\
\hline Intracellular calcium ion & $\begin{array}{l}\text { Determines receptor activation by measuring } \\
\text { intracellular } \mathrm{Ca}^{2+} \text { level with calcium-sensitive } \\
\text { dye, Fura- } 2 \text {. }\end{array}$ & $\begin{array}{l}\text { Grynkiewicz et al., 1985; Cullinan et al., 1994; } \\
\text { Bode et al., } 1999\end{array}$ \\
\hline \multicolumn{3}{|l|}{ Determination of incretin effects: } \\
\hline $\begin{array}{l}\text { Glucose tolerance test (GTT; oral GTT, } \\
\text { OGTT; intraperitoneal GTT, IPGTT; } \\
\text { intravenous GTT, IVGTT) }\end{array}$ & $\begin{array}{l}\text { Determines insulinotropic action of GLP-1 } \\
\text { analogs by measuring glucose level after their } \\
\text { administration. }\end{array}$ & Kreymann et al., 1987; Toft-Nielson et al., 1996 \\
\hline Insulin secretion & $\begin{array}{l}\text { Determines potency of GLP-1 analogs by } \\
\text { measuring insulin secretagogue action. }\end{array}$ & $\begin{array}{l}\text { Albano et al., 1972; Andersen et al., 1993; Goke } \\
\text { et al., 1993b; Toft-Nielson et al., 1996; Kjems } \\
\text { et al., 2003; Peyot et al., } 2009\end{array}$ \\
\hline
\end{tabular}

these stable GLP-1R agonists showed a high renal uptake, the potential of GLP-1R in visualizing $\beta$ cells has been demonstrated by PET and SPECT imaging of insulinoma with radiolabeled exendin-3 and exendin-4 (Gotthardt et al., 2006; Brom et al., 2010; Wild et al., 2010). As an excellent PET tracer, ${ }^{64} \mathrm{Cu}$ was used to label exendin-4, and the resulting ${ }^{64} \mathrm{Cu}$-labeled exendin-4 analog $\left({ }^{64} \mathrm{Cu}\right.$-DO3A-VERSUS-Cys ${ }^{40}$-exendin-4) demonstrated a feasibility for in vivo imaging of intraportally transplanted islets in mice by showing high and specific uptake in INS-1 tumors despite substantial renal uptake (Wu et al., 2011). Remarkably, $\left[\mathrm{Lys}^{40}(\mathrm{Ahx}-\right.$ DTPA- $\left.{ }^{111} \mathrm{In}\right) \mathrm{NH}_{2}$ ]-exendin-4 was successfully used to visualize autologous islets that have been transplanted into human muscle, proving a clinical potential of human $\beta$ cell imaging via GLP-1R (Pattou et al., 2010).

In addition to GLP-1R agonists described above, antagonists, such as exendin ${ }_{9-39}$, were also developed as $\beta$ cell imaging agents (Mukai et al., 2009). Radiolabeled at lysine residues with ${ }^{125}$ I-Bolton-Hunter reagent, exendin ${ }_{9-39}$ was examined for receptor specificity in vitro and in vivo. Accumulation of radioactive signals in $\beta$ cells was observed, although the resolution of the imaging technique was low.

Ahn and colleagues have developed PET-imaging agents based on the GLP-1 sequence to quantitate BCM 
(Ahn et al., 2011; Gao et al., 2011). As the endogenous GLP-1 suffers from rapid metabolic degradation, they introduced two lactam bridges to GLP-1, one in the $\mathrm{N}$-terminal region and the other near the $\mathrm{C}$ terminus. These two strategically placed lactam bridges were found to protect the cyclic peptides from proteolytic cleavages by NEP 24.11 (Murage et al., 2010). In addition, Ala at position 8 was replaced with D-Ala to prevent the DPP-4 degradation. These modifications not only enhanced metabolic stability of the GLP-1 analog, but also increased binding affinity to the receptor. Dynamic PET scans over 60 minutes showed a high pancreatic uptake of a bicyclic GLP-1 analog, [D-Ala $\left.\left.{ }^{8}\right]-\mathrm{c}^{2} \mathrm{Glu}^{18}, \mathrm{Lys}^{22}\right]-\mathrm{c}\left[\mathrm{Glu}^{30}, \mathrm{Lys}^{34}{ }^{34}\right.$ GLP-1 $1_{7-36}$, labeled with ${ }^{64} \mathrm{Cu}$ or ${ }^{68} \mathrm{Ga}$, which disappeared in streptozotocin-induced type 1 diabetic mice or was competitively displaced by coinjection of unlabeled exendin-4 (Manandhar et al., 2013). Ex vivo scans and histology were also carried out to further confirm the PET-imaging findings (unpublished data).

\section{Pharmaceutical Development and Therapeutics}

As noted above, GLP-1 is a gastrointestinal peptide hormone that is secreted from L cells scattered in the distal small intestinal mucosa. It is released physiologically when ingested nutrients reach that level of the bowel, typically reflecting a meal large enough to benefit from the incretin effect of this hormone. Among its other physiologic effects is the slowing of gastric emptying, again reflecting the benefit of titration of the rate of delivery of nutrients to the absorptive surface so as not to overwhelm the absorptive capacity of the intestine. This peptide has a very short half-life in the circulation, with proteolysis, particularly by DPP-4, and renal clearance having prominent roles. This endoprotease cleaves the amino-terminal dipeptide from endogenous GLP-1 peptides; GLP-1 $1_{7-36}$ amide or GLP-1 ${ }_{7-37}$ is cleaved to yield GLP- $1_{9-36}$ amide and GLP-1 $1_{9-37}$, respectively, which are four orders of magnitude less active than the natural hormone (Montrose-Rafizadeh et al., 1997). Recognition of this led to the earliest strategy to increase endogenous GLP-1 levels using DPP-4 inhibitors, rather than acting at GLP-1R. This was ultimately followed by the development of molecules having intrinsic GLP-1R agonist activity. As described below, most of these are peptides and their derivatives, but small-molecule agonists have also surfaced. All of them have potential importance for diabetes, obesity, and associated metabolic and cardiovascular complications. Other candidate applications are also discussed.

The earliest recognition that GLP-1 exhibited effects useful in the management of diabetes came from rodent studies (Gutniak et al., 1992; Nauck et al., 1993c). This was followed by a seminal study in which GLP-1 itself was administered s.c. over a 6 -week period in patients with T2DM (Zander et al., 2002). These patients displayed reduced levels of fasting glucose and $\mathrm{HbA1c}$, as well as improved insulin sensitivity and $\beta$ cell function. The proof-of-concept for the use of GLP-1 in diabetes demonstrated in this study was clear, yet major challenges existed that involved acceptable modes of administration and the need to improve duration of hormonal action.

There are now a wide variety of different peptidic GLP-1R agonists with variable durations of action and different advantages and disadvantages for clinical use. The first GLP-1R agonists to enter clinical use were peptide analogs and derivatives, as exemplified by exendin- 4 (Eng et al., 2014), liraglutide, albiglutide, and dulaglutide (Table 3). Unfortunately, these peptides require parenteral administration, due to instability in the proteolytic milieu of the digestive tract, making it impossible to deliver adequate drug reproducibly via oral route. Technology has advanced so much, however, that easy-to-use pens for s.c. administration has become quite common. Semaglutide is a potent peptide that was recently tested with s.c. administration in a phase 2 clinical study of 411 patients (Nauck at al., 2016), showing improvement in HbA1c levels by $1.7 \%$. This peptide is now being studied for oral dosing. This field has progressed so quickly that there are now even devices that allow longer-term activity being developed. Intarcia is an implantable device that releases exenatide over 612 months. This has been studied in two phase 3 trials (Mullard, 2014).

We have finally entered an era in which smallmolecule nonpeptidic GLP-1R agonists are now available (Fig. 7), although it still poses a tremendous challenge to make them adequately potent and bioavailable (Yang et al., 2015a). Most of these are thought to act within the helical bundle of GLP-1R. Crystallization of two members of the class B GPCR subfamily in 2013 (Hollenstein et al., 2013; Siu et al., 2013) provided a clue to why development of nonpeptidic modulators has been so difficult. Unlike the class A GPCRs that have tight helical bundles with a well-defined pocket for small-molecule ligands, the class B helical bundles appear to be much more open without defined small-molecule docking sites. Recently, one of the small-molecule agonists (BETP) had its site of action determined as covalently binding to a cysteine residue in intracellular loop 3 on the cytosolic side of the plasma membrane (Nolte et al., 2014), a molecular mechanism that was a major surprise, without precedent in other members of this subfamily. A molecule from TransTech Pharma, TTP054, is presently undergoing phase 2 clinical trials (Mullard, 2014).

\section{A. Peptidic Analogs}

1. GLP-1 Analogs. Several GLP-1 analogs have successfully reached the market and become an important treatment of obesity and T2DM today (Table 3). Several reviews have been published during the past that describe the approved and emerging GLP-1R agonists and also summarize the most important clinical trials as well 
as toxicological considerations (Lund et al., 2011; Madsbad et al., 2011; Garber, 2012; Meier, 2012; Montanya, 2012; Lorenz et al., 2013; Trujillo and Nuffer, 2014; Trujillo et al., 2015; Madsbad, 2016; Zaccardi et al., 2016).

Taspoglutide (BIM-51077; Roche, Basel, Switzerland) is a potent GLP-1 analog with $93 \%$ homology to human GLP-1 $1_{7-36}$ amide as it has two amino acid substitutions in positions 8 and 35 with aminoisobutyric acid (Aib). Taspoglutide is protected against DPP-4 degradation by the $\mathrm{Aib}^{8} / \mathrm{Aib}^{35}$ substitution but is still cleared rapidly by the kidney. A sustained-release formulation that contains zinc-chloride and precipitates as a sub cutis deposition was developed. This once-weekly dosing formulation of taspoglutide has shown promising antidiabetic efficacy in clinical trials. Unfortunately, the analog was discontinued in phase 3 clinical trials due to severe gastrointestinal side effects (Retterstol, 2009; Dong et al., 2011; Garber, 2012).

The GLP-1 human serum albumin fusion protein, albiglutide, also known as albugon, discovered by Genentech (South San Francisco, CA) and developed by GlaxoSmithKline (Brentford, UK), was approved by the Food and Drug Administration (FDA) in 2014. To have a free N-terminal, which is important for GLP-1 activity, the peptide was fused via the $\mathrm{C}$ terminus to albumin. The construct is composed of a tandem repeat of $\mathrm{Ala}^{8}$ to $\mathrm{Gly}^{8} \mathrm{GLP}-1_{7-36}$ that is fused to the $\mathrm{N}$ terminus of human albumin. Gly ${ }^{8}$ GLP-1 was used to protect for DPP-4 degradation of the $\mathrm{N}$ terminus. The plasma halflife was extended to about $6-8$ days, which made this fusion construct applicable for once-weekly dosing. One drawback relates to a significantly reduced potency that was probably due to a combination of the $\mathrm{Gly}^{8}$ modification and the covalent attachment to the larger protein human serum albumin. The tandem repeat of GLP-1 was used to obtain longer distance between albumin and the distal GLP-1 peptide, but the affinity for GLP$1 \mathrm{R}$ is $0.61 \mathrm{nM}$ compared with $0.02 \mathrm{nM}$ of the native peptide. The approved initial dose to treat T2DM is $30 \mathrm{mg}$ given s.c. once per week, which may be increased to $50 \mathrm{mg}$ on the same day of each week if the glycemic response is inadequate (Kim et al., 2003; Tomkin, 2009; St Onge and Miller, 2010).

Dulaglutide (LY2189265; Eli Lilly, Indianapolis, IN) is a GLP-1R agonist fused to a modified Fc fragment of immunoglobulin that acts as a carrier to secure long residence time by reduced in vivo clearance. The sequence of the fusion protein $\mathrm{Gly}^{8} \mathrm{Glu}^{22} \mathrm{Gly}^{36}{ }_{-} \mathrm{GLP}-1_{7-37^{-}}$ $\left(\mathrm{Gly}_{4} \mathrm{Ser}\right)_{3} \mathrm{Ala}-\mathrm{Ala}{ }^{234,235} \mathrm{Pro}^{228}{ }_{-} \mathrm{IgG}_{4}-\mathrm{Fc}$ is modified in the $\mathrm{N}$ terminus of GLP-1 to protect against DPP-4 cleavage $\left(\mathrm{Gly}^{8}\right)$, and the $\mathrm{IgG}_{4} \mathrm{Fc}$ is modified to reduce interaction with high-affinity Fc receptors and avoid antibodydependent cell-mediated cytotoxicity. The linker $\left(\mathrm{Gly}_{4-}\right.$ Ser) ${ }_{3}$ Ala was carefully selected to retain high GLP-1R affinity as direct fusion dramatically reduced in vitro activity by $\sim 95 \%$. The half-life is above 1.5 days in rats and more than 2 days in monkeys. Dulaglutide was approved by the FDA in 2014 for once-weekly dosing of $0.75 \mathrm{mg}$ s.c. (Glaesner et al., 2010).

There is still a need for optimization of once-weekly GLP-1 analogs because both albiglutide and dulaglutide are less efficacious than liraglutide with respect to weight loss (Dungan et al., 2014; Pratley et al., 2014).

The next generation of GLP-1 analogs using the reversible albumin affinity is represented by semaglutide (Novo Nordisk, Bagsværd, Denmark) currently in phase 3 clinical trials. This analog $\mathrm{Aib}^{8} \mathrm{Lys}^{26} \mathrm{Arg}^{34}-\mathrm{GLP}-1_{7-37}$ has a more complex side chain composed of hexadecandinoyl attached to Lys ${ }^{26}$ via an L- $\gamma$-glutamic acid linker and a small hydrophilic spacer. The $\mathrm{Aib}^{8}$ was introduced to improve the DPP-4 stability beyond that of liraglutide, and the new side chain increased the albumin affinity, resulting in a half-life in humans long enough for once-weekly dosing. In a phase 2 study over 12 weeks, semaglutide dose-dependently reduced HbA1c and body weight, with higher doses being more effective than liraglutide (Nauck and Sesti, 2012; Trujillo and Nuffer, 2014; Lau et al., 2015).

2. Exenatide Experience. In 1993, exendin-4 was isolated from the salivary glands of the gila monster (Heloderma suspectum) and found to be highly active on GLP-1R (Goke et al., 1993a). Exendin-4 is a 39 amino-acid peptide that shares approximately 50\% amino acid sequence identity with human GLP-1. The discovery of the antidiabetic effects of exendin-4 increased the interest in this peptide, and several pharmaceutical companies thus focused on its clinical development as well as on discovery efforts in exendinbased GLP-1 analogs.

In 2005, the first GLP-1R agonist exenatide (AstraZeneca, Cambridge, UK) was approved by the FDA for treatment of T2DM. Exenatide, as well as other GLP-1R agonists, reduces blood glucose through multiple mechanisms, including enhancement of glucose-dependent insulin secretion, suppression of excess glucagon secretion, reduction of food intake, and slowing of gastric emptying. It is administered s.c. twice daily with doses between 5 and $10 \mu \mathrm{g}$ (Cvetkovic and Plosker, 2007).

The very low therapeutic dose of exenatide made it an excellent candidate for an extended release formulation. Amylin Pharmaceuticals (San Diego, CA), Eli Lilly, and Alkermes (Dublin, Ireland) developed a formulation to prolong exenatide release, using biodegradable polymeric microspheres that entrap exenatide. The microspheres consist of exenatide incorporated into a matrix of poly (D,L-lactide-co-glycolide), which is a common biodegradable medical polymer with a history of safe use in humans. The final formulation was approved in 2011 as Bydureon for once-weekly dosing (Kim et al., 2007; Malone et al., 2009).

Lixisenatide (Sanofi, Paris, France) is a once-daily short-acting GLP-1R agonist used in the treatment of T2DM. The discovery of lixisenatide was inspired by the development of structure-inducing probe technology, 
TABLE 3

Peptidic GLP-1R agonists that have been launched or are in late-stage clinical trials

\begin{tabular}{|c|c|c|c|c|}
\hline Analog & Description & Company & Dosing Regimen & Status \\
\hline Exenatide & Exendin-4 & Amylin/Eli Lilly; AstraZeneca & Twice daily & Launched \\
\hline Exenatide once weekly & Exendin-4 & $\begin{array}{l}\text { Amylin/Alkermes/Eli Lilly; } \\
\text { AstraZeneca }\end{array}$ & Once weekly & Launched \\
\hline Lixisenatide & Exendin-4 analog & Sanofi-Aventis & Once daily & Launched \\
\hline Liraglutide & GLP-1 analog linked with a fatty acid & Novo Nordisk & Once daily & Launched \\
\hline Albiglutide & GLP-1 analog fused to albumin & GlaxoSmithKline & Once weekly & Launched \\
\hline Dulaglutide & GLP-1 analog fused to Fc & Eli Lilly & Once weekly & Launched \\
\hline Semaglutide & GLP-1 analog linked with a fatty acid & Novo Nordisk & Once weekly & Phase 3 \\
\hline Semaglutide (NN9924) & GLP-1 analog linked with a fatty di-acid & Novo Nordisk & Oral & Phase 2 \\
\hline Taspoglutide & GLP-1 analog formulated for sustained release & Roche & Once weekly & Suspended \\
\hline
\end{tabular}

which improves the half-life of structure-inducing probe conjugates compared with the parent peptide by enhancing stability and, as a consequence, creating resistance to proteolytic degradation. Lixisenatide, desPro $^{36}$-exendin- $4_{1-39}-$ Lys $^{6}$ amide, comprises 44 amino acids and is based on the exendin- 4 sequence with a proline deletion in position 36 and C-terminal extension with six lysines. Lixisenatide has an extended in vivo half-life of 91 minutes after i.v. administration (in rabbits) compared with 43 minutes for exendin-4. Lixisenatide was approved in 2013 and has a substantial sustained effect on gastric emptying and postprandial glucose excursions that may be related to the relatively shorter half-life. The combination with basal insulin to lower fasting plasma glucose is clinically valuable and could differentiate lixisenatide from other GLP-1R agonists, especially from those longacting ones with little effect on gastric emptying and postprandial glucose (Werner et al., 2010; Christensen et al., 2014; Rosenstock et al., 2014).

3. GLP-1 Mimetics. A novel class of 11-amino-acid GLP-1R agonists was discovered that consist of an analog of only the first 9 amino acids of GLP-1, in which the remaining 21 amino acids have been truncated and attached to an unnatural biphenyl diamino acid at the $\mathrm{C}$ terminus. The optimization of the sequence with several unnatural amino acids provided 11-mer GLP-1 mimetics with similar in vitro potency as the natural 30-mer peptide. The optimized 11-mers (e.g., BMS686117) were reported to reduce plasma glucose and increase plasma insulin concentrations in diabetic $o b /$ $o b$ mice after a single relative high s.c. dose of the peptide (Mapelli et al., 2009; Haque et al., 2010). Based on NMR studies, the structures of these 11-mer peptides were determined and used to design lactam- and disulfidebased cyclic constrained peptides that retained high GLP$1 \mathrm{R}$ potency (Hoang et al., 2015).

BMS-686117 has a relatively shorter half-life of about 2 hours in dogs, and, accordingly, a sustained release formulation is required for clinical use. Formulation with $\mathrm{Zn}$ (II) forms a poorly water-soluble adduct such that the BMS-686117 content and reversible release of which can be tailored by varying the $\mathrm{Zn}$ :peptide ratio. An in vivo pharmacokinetics study in dogs of an optimized sustained release formulation concluded that a minimal initial burst and constant release are applicable to once-daily dosing (Qian et al., 2009).

\section{B. Nonpeptidic Modulators}

Even though more than two decades have passed since the cloning of the GLP-1R (Thorens, 1992), very few nonpeptidyl-based GLP-1R agonists have been published. One might speculate on the reason: the ligandreceptor interaction leading to activation requires multiple interfaces that are difficult to mimic with a small molecule. The majority of the nonpeptidyl-based GLP-1R agonists described in the literature are receptor modulators that bind to allosteric sites and do not compete with GLP-1 in the orthostatic site. Many such GLP-1R agonists were only mentioned in patents and are not included in this section, as their experimental data have not been peer-reviewed. There are also small numbers of nonpeptidic GLP-1R antagonists, and these will be discussed in the last part of this section. One review article is also referred concerning nonpeptidic GLP-1R modulators (Willard et al., 2012a).

To date, only one nonpeptidic GLP-1R agonist has entered into clinical trials. The compound has not been specifically disclosed, but a publication from the company described that it stimulated glucose-dependent insulin release in isolated islets from rodents and improved glycemic control in an oral glucose tolerance test (Gustavson et al., 2014).

Boc5, a substituted cyclobutane with four chiral centers, is among the few nonpeptidic GLP-1R agonists that have been comprehensively studied. It was discovered during a high-throughput screening against 48,160 small molecules using a luciferase reporter assay in HEK293 cells expressing the rat GLP-1R (Chen et al., 2007). Boc5 is the only nonpeptidic GLP-1R agonist that has been shown to compete for ${ }^{125}$ I-GLP-1 in binding assays and to be functionally antagonized by exendin $_{9-39}$, thus acting like the natural peptide ligand.

The antidiabetic effect of Boc5 was demonstrated in various animal models. A 4-week dose-response study was performed in $d b / d b$ mice with daily i.p. dosing of Boc5 at 0.1-3 mg. There was a dose-dependent decline in $\mathrm{HbA1c}$ during the treatment that continued to decrease until 2-3 weeks after the last dose. In contrast, Boc5 had no effect on HbA1c in nondiabetic C57BL/6J 
<smiles>CCc1cc(OC)ccc1-c1ccc(C[C@H](NC(=O)[C@H](CC(=O)O)NC(=O)[C@H](CO)NC(=O)[C@H](NC(=O)[C@](C)(Cc2ccccc2F)NC(=O)[C@H](NC(=O)CNC(=O)[C@H](CCC(=O)O)NC(=O)C(C)(C)NC(=O)[C@H](C)Cc2c[nH]cn2)[C@@H](C)O)[C@@H](C)O)C(=O)N[C@@H](Cc2ccc(-c3ccccc3C)cc2)C(N)=O)cn1</smiles>

Compound 1

Compound 2<smiles>CCS(=O)(=O)c1cc(-c2ccc(Cl)cc2)cc(C(F)(F)F)c1</smiles>

Compound A<smiles>CCS(=O)c1cc(-c2cccc(OCc3ccccc3)c2)cc(C(F)(F)F)c1</smiles>

Compound B, BETP

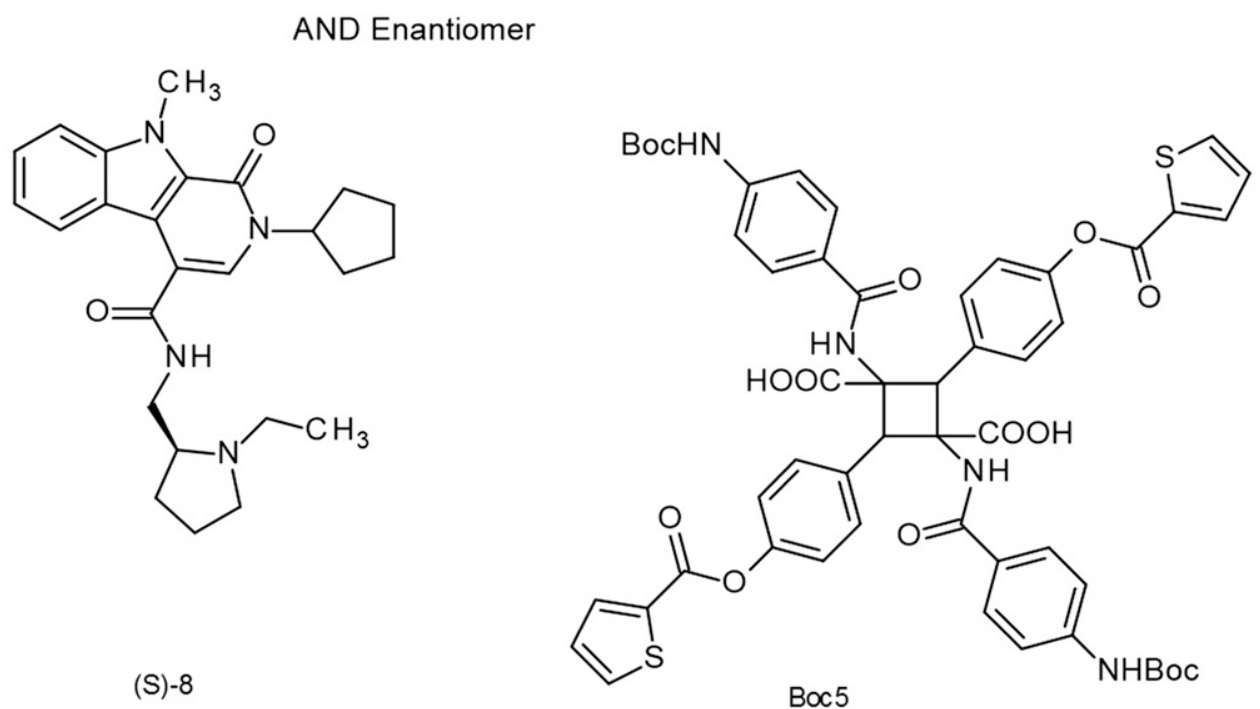

Fig. 7. Nonpeptidic GLP-1R modulators and peptide mimetics. Liraglutide (Novo Nordisk) was the first approved human GLP-1 analog to teat diabetes (European Union, 2009; United States, 2010) and obesity (United States, 2014; European Union, 2015). Liraglutide has 97\% sequence 
(wild-type) mice. Boc5 also inhibited food intake and decreased body weight of $d b / d b$ mice by up to $16 \%$ during the study with reduced fat mass. In addition, it was demonstrated that Boc5 decreased the elevated levels of fasting insulin in $d b / d b$ mice, and the insulin sensitivity was improved after 4 weeks of treatment. Thus, the effects of Boc5 seen both in vitro and in vivo suggest that this compound has similar pharmacological properties as peptidic GLP-1R agonists. Medicinal chemistry efforts were made to understand the SAR, and one analog, WB4-24, was found to be more potent in vitro compared with Boc5. WB4-24 was shown to have beneficial effects on glucose homeostasis and body weight in diet-induced obese mice ( $\mathrm{Su}$ et al., 2008; He et al., 2010, 2012; Liu et al., 2012).

After an unsuccessful screening of 500,000 discrete small molecules with a GLP-1 competitive binding assay, a substituted quinoxaline was identified in subsequently performed functional screening of 250,000 compounds. This hit as well as some closely related analogs activated the formation of cAMP in BHK cells expressing the GLP-1R (Fig. 7, compounds 1 and 2). However, it did not compete with ${ }^{125}$ I-GLP-1 in a binding assay, but augmented the binding of the tracer. Furthermore, its functional effect could not be antagonized by exendin 9-39. $^{2}$ Functionality counter-screening analysis showed that the quinoxalines were inactive in BHK cells expressing GIPR, GLP-2 receptor, and GCGR. They were capable of releasing insulin from wild-type mouse islets, but not from islets isolated from GLP-1R knockout mice. These quinoxalines were thus concluded to be selective GLP-1R ago-allosteric modulators. Further SAR studies around these initial leads as well as some of the later identified nonpeptidic modulators have all been published (Knudsen et al., 2007; Teng et al., 2007; Coopman et al., 2010; Irwin et al., 2010; Koole et al., 2010; Cheong et al., 2012; Li et al., 2012a).

Pyrimidine-based ago-allosteric modulators (Fig. 7, compounds A and B) of GLP-1R were found by screening of a small library enriched with relevant pharmacophores for GPCRs. The screening was performed in HEK293 cells coexpressing hGLP-1R and a cAMP response element luciferase reporter. Like the quinoxalinebased modulators, the pyrimidines did not compete with ${ }^{125}$ I-GLP-1 in a binding assay and the functional effect was not antagonized with exendin $9-39$. They were shown to enhance GLP-1-induced cAMP signaling and insulin release in isolated rat islets. Compound $\mathrm{B}$ (BETP) increased plasma insulin levels in a glucose tolerance study using Sprague Dawley rats as well as hyperglycemic clamped Spraqgue Dawley rats. One drawback for further development of both quinoxaline- and pyrimidinebased leads is the chemical instability in the presence of nucleophiles (Sloop et al., 2010).

Recently, a tricyclic pyridoindole-based series of GLP-1R allosteric modulators was identified by highthroughput screening measuring intracellular calcium mobilization in the presence of low levels of GLP-1 or glucagon. These compounds were shown to potentiate glucose-dependent insulin release in primary mouse islets, but no in vivo studies were described (Morris et al., 2014).

The potential use of GLP-1R allosteric modulators to enhance the effect of endogenous GLP-1 was studied in vivo. An i.v. glucose tolerance study was carried out in which coadministration of BETP to rats dosed with subsaturating concentrations of oxyntomodulin markedly increased the insulinotropic effect of oxyntomodulin (Willard et al., 2012b).

\section{Currently Approved Clinical Applications}

A number of GLP-1R agonists have been approved for clinical use to improve glycemic control in adults with T2DM. Diet and exercise are the expected initial approach to treat these patients, with GLP-1R agonists prescribed as second-line drugs for patients who are refractory to the current standard of care. GLP-1R agonists have no current role in the management of patients with type 1 diabetes, and should be avoided in those patients having a history of pancreatitis or medullary thyroid cancer. The use of this category of drugs in T2DM reflects their direct effects on the $\beta$ cells within the pancreatic islets to increase insulin biosynthesis and secretion, to stimulate cell proliferation, and to reduce apoptosis. A key benefit of GLP-1R agonists is the glucose-dependent effects on the $\beta$ cell, with reduction in blood glucose only in patients exhibiting hyperglycemia. This eliminates the hypoglycemia risk of many other antidiabetic drugs. In fact, GLP-1R agonists have now largely replaced the use of thiazolidinediones (e.g., rosiglitazone). Adverse responses such as peripheral edema, weight gain, congestive heart failure, and osteoporosis associated with the latter are not observed in patients treated with GLP-1R agonists (Drucker et al., 2010). GLP-1R agonists also exhibited cardiovascular benefits, such as lowering blood pressure, improving lipid profiles, and possibly even enhancing cardiac contractility and

homology to human GLP-1 and was designed to reversibly bind to albumin by attachment of palmatic acid via a L- $\gamma$-glutamic linker to lys ${ }^{26}$ in Arg ${ }^{34}$ GLP-1 $1_{7-37}$. The modification of $\mathrm{Lys}^{34}$ to $\mathrm{Arg}^{34}$ made it possible to produce Arg ${ }^{34}$ GLP-1 $1_{7-37}$ in yeast, followed by acylation of Lys ${ }^{26}$. The native GLP-1 peptide has a half-life of approximately 2 minutes due to rapid cleavage of GLP- $1_{7-37}$ to GLP-19-37 by DPP-4 (Deacon et al., 1995a,b; Vilsboll et al., 2003). Liraglutide comprises the natural GLP-1 N terminus, but has a half-life of about 11 hours after s.c. dosing to humans combined with a delayed absorption from sub cutis that gives a pharmacokinetic profile applicable for once-daily administration. The reason for extended circulation is due to reversible albumin binding that protects from DPP-4 degradation and glomerular filtration, whereas the delayed absorption is explained by the ability of liraglutide to form heptamers by self-assemble controlled by the fatty acid side-chain at position 26 . Liraglutide is well tolerated and capable of substantially improving glycemic control with low risk of hypoglycemia and weight loss benefit (Knudsen et al., 2000; Knudsen, 2004; Madsen et al., 2007; Steensgaard et al., 2008; Dharmalingam et al., 2011; Wang et al., 2015). 
endothelial function. In addition to its action on islet $\beta$ cells, this hormone exerts effects on the following: 1) pancreatic $\alpha$ cells to reduce glucagon secretion and hepatic glucose output; 2) the cardiovascular system to elevate glucose utilization and protect the heart and vasculature against inflammation; 3) the brain to decrease food intake; and 4) the gut indirectly to slow gastric emptying.

1. Selection. As the number of GLP-1R agonists in clinical use and trials expands, it will become clearer whether there are advantages of one drug over another in a specific clinical setting. We have already observed differences in the frequency of nausea, presumably reflecting the reduced gastric emptying, among these drugs. There is also variation in the pathway of inactivation and excretion of these drugs, making the selection of agent in patients with renal insufficiency important. Although biased agonism is now recognized for some of these agonists, there is still insufficient information on the broad pharmacodynamics properties of these drugs to differentiate the contribution of biased agonism from pharmacokinetic behavior to differential clinical responses, side effects, or toxicities, although this is likely to be an important consideration in future therapeutic development.

A very practical and useful categorization of GLP-1R agonists is based on their duration of action. The shortest-acting agents, like exenatide and lixisenatide with half-lives of $2-5$ hours, seem to exert their beneficial effects predominantly by slowing gastric emptying and thereby reducing the rate of carbohydrate delivery, digestion, and absorption, leading to normal postprandial glucose levels. Of course, other nutrients also empty more slowly, and this has resulted in lower postprandial levels of free fatty acids and triglycerides, which has some metabolic benefits. For instance, decreased levels of chylomicrons are desired in patients with diabetes or metabolic syndrome. As the duration of GLP-1R agonist action is extended with peptides like albiglutide, dulaglutide, long-acting release exenatide, or liraglutide (half-lives of 12 hours to several days), the impact on blood glucose levels is enhanced as insulinotropic action and activity to reduce glucagon become more prominent than the effect on gastric emptying. Such prolonged activities have also been associated with increased side effects, such as diarrhea, tachycardia, immune responses, and injection site reactions. Of interest, nausea and vomiting appear to have greater incidence with the shorter-acting peptides. The longeracting agents have typically provided more consistent effects on GLP-1R activation and blood glucose levels. Because the insulinotropic action of GLP-1 is dependent on the presence of hyperglycemia, long duration activity does not increase the risk of hypoglycemia. Therefore, long-acting release formulations have obvious advantages if the goal is to lower early morning glucose levels, because they retain efficacy throughout the night.
It is curious whether the induction of nausea, which is believed to be a result of slowed gastric emptying, is similar in the short-acting and longer-acting GLP-1R agonists, yet this seems to be attenuated quickly in the longer duration agonists and may take weeks to months to improve for the shorter duration agonists. Such a phenomenon most likely reflects differences in desensitization of GLP-1R, but the underlying mechanism has not been carefully studied. Although the weight loss effect seen with some of these GLP-1R agonists was thought to be related to slowed gastric emptying, the long-acting agents seem to result in greater weight reduction than the short-acting ones, suggesting certain other mechanism is dominant. This might relate to reduced appetite, potentially following GLP-1 action in the hypothalamus or other CNS regions.

2. Efficacy. The first phase 2 trial to study a GLP-1R agonist in T2DM was started in 1999, comparing twicedaily administration of exenatide to saline (Kolterman et al., 2003). Not only was the exenatide effective in reducing glucose levels, but it did not induce hypoglycemia in patients who were on insulin therapy. Although exenatide was approved by the FDA for use in diabetes in 2005 , concerns about hypoglycemia in insulinrequiring patients persisted, and this was finally studied in 2008 (Buse et al., 2011). The dramatic observations of enhanced reduction in HbA1c and no increase in hypoglycemic episodes, as well as weight loss, formally launched a new series of clinical studies to follow. A meta review published in Lancet in 2014 (Eng et al., 2014) chose 15 of 2905 studies that represented 4348 subjects to evaluate. HbA1c levels were decreased by an average of $0.44 \%$, and weight was reduced by an average of $3.22 \mathrm{~kg}$, without any increase in hypoglycemic episodes. Of note, the levels of HbA1c were not different using GLP-1R agonist with boluses of insulin, but the frequency of hypoglycemic episodes was significantly reduced with the former.

Short-acting GLP-1R agonists known to better control postprandial glucose levels have not been directly compared with that of long duration administered with basal insulin. Each has theoretical advantages and perhaps should be chosen based on the characteristics of the patient. Because long-acting agents can be given with short-acting insulin at meal time now, another important option exists, but needs to be evaluated.

The DURATION-3 trial (Diamant et al., 2014) compared long-acting release exenatide administered once per week to insulin glargine (a long-acting, man-made version of human insulin) in 456 patients with T2DM who had not achieved satisfactory glucose control with maximal tolerated doses of oral agents. Exenatide was able to better manage the blood glucose, with HbA1c levels being reduced by $1.01 \%$, whereas glargine plus oral agents only reduced this by $0.81 \%$. Again, the most notable observation was a much lower incidence of hypoglycemia in patients taking the GLP-1R agonist. 
The AWARD-4 study did use dulaglutide injected only once per week and rapid-acting insulin with each meal to demonstrate improved levels of HbA1c than were observed in basal and bolus insulin administration (Jendle et al., 2014). Fixed-dose combinations of GLP$1 R$ agonist and basal insulin have substantial appeal for convenience, although, at the current time, expense is a major concern. The DUAL-1 and DUAL-2 studies exhibited a $1.9 \%$ reduction in $\mathrm{HbA1c}$ in patients who followed a titration algorithm with a pen delivery device (Buse et al., 2011; Gough et al., 2014).

3. Benefits. In addition to its action on blood glucose, mediated largely by impact on the pancreatic islet $\beta$ and $\alpha$ cells, GLP-1R agonists have been shown to display favorable cardiovascular effects. This includes improvements in serum lipids, myocardial contractility, and endothelial function (Mudaliar and Henry, 2010). The effects on lipids are likely to reflect slowed gastric emptying and reduced delivery for absorption, as well as impact on hepatic and fat cell metabolism. Presumably, the direct cardiovascular benefit was achieved via abundant levels of GLP-1R expression in cardiomyocytes and blood vessels (Nolte et al., 2014). Experiments in rodents showed that GLP-1R agonists can reduce myocardial infarct size and improve left ventricular function, including better wall motility and ejection fractions (Nikolaidis et al., 2004b; Noyan-Ashraf et al., 2009). There also exists encouraging evidence in rodents of the neuroprotective role of GLP-1 for AD, PD, and even stroke (Harkavyi et al., 2008; McClean et al., 2011; Teramoto et al., 2011), although this has not been followed up by clinical studies.

A recent meta-analysis explored the impact of GLP$1 \mathrm{R}$ agonists on lipids in clinical trials (Sun et al., 2015). Thirty-five trials with 13 treatments lasting a minimum of 8 weeks were included, and GLP-1R agonists were associated with modest improvements in total cholesterol, low-density lipoprotein cholesterol, and triglyceride levels, whereas there was no change in high-density lipoprotein cholesterol. It is not yet clear whether this translates into an improvement in cardiac and vascular events. It follows that a latest retrospective review of macrovascular outcomes in patients with T2DM who had been treated with insulin alone or with GLP-1R agonists (Paul et al., 2015) suggested that heart failure, myocardial infarction, and stroke were less common in patients who were taking the drug. Although such epidemiologic studies need to be interpreted carefully, this is inspiring and clearly worthy of prospective evaluation. A nested case-control study was also recently performed (Gejl et al., 2015). T2DM patients who developed one or more of these cardiovascular problems were compared with control patients with diabetes who did not develop such cardiovascular events. More than 10,000 patients were included, and 1,947 had one or more of these events. Liraglutide was associated with significant risk reduction, with the degree of reduction being dose- and duration-dependent. Another retrospective cohort study also showed a reduction in heart failure in patients with diabetes (Velez et al., 2015). More than 19,000 adult patients with diabetes were studied and matched with twice as many controls, and 1,426 patients with diabetes used GLP-1R agonists. This was also encouraging, but only points toward the need for prospective studies.

There is increasing excitement about the potential role for fixed-dose combinations of a long-acting insulin preparation and a GLP-1R agonist as an adjunct to diet and exercise to improve glycemic control in adults with T2DM. Two such preparations have recently been recommended for FDA approval by advisory committees, based on portfolios of clinical trials, including doubleblinded designs. These include IDegLira from Novo Nordisk, combining Tresiba (insulin degludec) and Victoza (liraglutide) into a once-daily injectable product (http://www.fda.gov/downloads/advisorycommittees/ committeesmeetingmaterials/drugs/endocrinologicandmetabolicdrugsadvisorycommittee/ucm502074.pdf), and iGlarLixi from Sanofi, combining Lantus (insulin glargine) and Lyxumia (lixisenatide) into a similar product (http://www.fda.gov/downloads/advisorycommittees/ committeesmeetingmaterials/drugs/endocrinologicandmetabolicdrugsadvisorycommittee/ucm502559.pdf). Both of the component drugs in IDegLira and the insulin glargine in iGlarLixi are already FDA approved, whereas lixisenatide is currently undergoing independent regulatory review. The efficacy of both combination products was superior to that of the components alone, leading to the successful outcomes. The safety of them appears to be consistent with that of the individual components, and there are indications that some side effects like nausea and weight gain may actually be less in the combination preparations than for some individual components. It seems likely that at least one of these products or something alike will achieve a prominent role in therapy soon.

\section{Potential Applications for Other Diseases}

1. Neurologic Disorders. The expression of GLP-1R in multiple sites of the nervous system and the involvement of GLP-1 in satiety and food intake regulation are well-established (II. Glucagon-Like Peptide-1). Because this incretin was also shown to improve learning in rats (Oka et al., 2000) and exert neurotrophic or neuroprotective effects when given intracerebroventricularly, its potential therapeutic value for $\mathrm{AD}, \mathrm{PD}$, and pain has been investigated (During et al., 2003; McClean et al., 2011; Gong et al., 2014b).

$\mathrm{AD}$ is characterized by progressive cognitive decline with a defined neuropathology of $\mathrm{A} \beta$ production and tau hyperphosphorylation (Claeysen et al., 2012). T2DM has been identified as a risk factor for $\mathrm{AD}$, and an epidemiologic study indicated that $65 \%$ of T2DM patients showed evidence of featured $\mathrm{AD}$ plaques in their autopsied brains (Bassil et al., 2014), indicating that 
insulin desensitization in the periphery may be a factor in initiating or accelerating the development of neurodegenerative processes. It has led to the notion that drugs developed for the treatment of T2DM may be beneficial in modifying the pathophysiology of $\mathrm{AD}$. In preclinical models of $\mathrm{AD}$, GLP- 1 decreases $\mathrm{A} \beta$ toxicity in vitro (During et al., 2003; Perry et al., 2003). A follow-up study demonstrated that GLP-1 and exendin4 could protect cultured rat hippocampal neurons against glutamate-induced apoptosis (Perry et al., 2003). Furthermore, $\mathrm{Val}^{8}$ GLP-1 blocked synaptic degradation and rescued synaptic plasticity in the hippocampus (Gengler et al., 2012). The neuroprotection effect of GLP-1 was further displayed in studies in which treatment with liraglutide, lixisenatide, or geniposide in the transgenic amyloid precursor peptide/presenilin 1 mice produced improvements in synaptic plasticity and reduced neuronal damage, tau hyperphosphorylation, microglial activation, plaque, and oligomer formation in the CNS (Perry et al., 2002; McClean et al., 2011; Lv et al., 2014; McClean and Holscher, 2014). These GLP-1 analogs also attenuated memory deficits, restored impaired signaling within the Akt/GSK- $3 \beta$ pathway, and reversed elevated levels of reactive oxygen species in amyloid precursor peptide/presenilin 1 mice. The recent reported effect of liraglutide on an early-stage sporadic $\mathrm{AD}$ model supports the notion that liraglutide-induced GLP-1R activity might be a viable target in $\mathrm{AD}$ therapy (Hansen et al., 2015).

PD is typified by a loss of dopaminergic neurons and cellular degeneration in the striatum (Le et al., 2009). Epidemiologic data suggest an association between T2DM and some neurodegenerative disorders, such as PD (Pressley et al., 2003). In patients with PD, it was found that the levels of insulin receptors were markedly reduced in the basal ganglia and the substantia nigra (Moroo et al., 1994). Furthermore, increased IRS2 phosphorylation, a marker of insulin resistance, was found in the basal ganglia of the 6-hydroxydopamine lesion rat PD model (Morris et al., 2008). An earlier report demonstrated that in high-fat-diet-fed rats, insulin resistance was accompanied by attenuated dopamine release and diminished dopamine clearance in the basal ganglia (Morris et al., 2011). Neuropathological studies in patients with PD also revealed that loss of insulin receptor immunoreactivity and mRNA coincides with loss of tyrosine hydroxylase mRNA (the rate-limiting enzyme in dopamine synthesis) (Takahashi et al., 1996).

GLP-1 and exendin-4 have been shown to exert a neurotrophic effect on 6-hydroxydopamine-treated dopamine neurons ( $\mathrm{Li}$ et al., 2010). A growing body of evidence now exists to support the neurotrophic properties of GLP-1: activating GLP-1R-signaling pathways in neurons leads to proliferation and differentiation of cells from precursors into neurons, thereby drawing a striking similarity between cellular responses in pancreatic $\beta$ cells and neurons (During et al., 2003). Indeed, such a protective action was observed in rodent models of PD: GLP-1 and its mimetics were effective to protect tyrosine hydroxylase-positive dopaminergic neurons and to preserve dopamine levels in 1-methyl-4-phenyl1,2,3,6-tetrahydropyridine-treated animals. Treatment with a GLP-1R agonist resulted in improved motor function, as demonstrated by rotarod and pole tests (Kim et al., 2009; Li et al., 2009). Recently, a single-blind clinical trial of exendin-4 in PD patients showed that exenatide was well tolerated, and the treated group exhibited clinically relevant improvements across motor and cognitive measures compared with the control group (Aviles-Olmos et al., 2013).

In a study to investigate glucoregulatory effects of exendins, a research group in Shanghai serendipitously discovered that GLP-1 produced a potent antinociception in the formalin test (Gong et al., 2011). They further examined the inhibitory role of the spinal GLP-1R signaling pathway in pain hypersensitivity states and its mechanism of action. Specific expression of GLP-1R on the spinal dorsal horn microglia was found, and the efficacy as well as potency of GLP-1, exenatide, and geniposide on antinociception in a variety of animal models of pain hypersensitivity versus acute nociceptive responses were evaluated (Gong et al., 2014b). The involvement of GLP-1 in antinociception was also verified by a nonpeptidic GLP-1R agonist (WB4-24) indicating that the effect is mediated by GLP-1R (Gong et al., 2014a; Fan et al., 2015).

2. Oncological Association. As previously described above, GLP-1-based therapy is a long-term approach to the control of metabolic disorders exemplified by T2DM, whereby chronic adverse events become a major concern, including an increased risk of cancer (Esposito et al., 2012). Pancreas and thyroid are the main tissues of the concern.

Since the first case report of exenatide-induced pancreatitis in 2006 (Denker and Dimarco, 2006), increasing numbers of controversial observations were reported associated with the risk of pancreatitis when treating T2DM patients with GLP-1R agonists or DPP-4 inhibitors. Acute pancreatitis may potentially progress to chronic pancreatitis and ultimately develop pancreatic cancer (Yachida et al., 2010; Nauck and Friedrich, 2013). Safety alerts have been issued by the FDA for exenatide and sitagliptin in 2008 and 2009, respectively, for patients who have a history of pancreatitis or current symptoms suggestive of pancreatitis. In 2013, a workshop sponsored by the National Institute of Diabetes and Digestive and Kidney Diseases and the National Cancer Institute on Pancreatitis-Diabetes-Pancreatic Cancer was held in Bethesda, MD (Andersen et al., 2013). Based on current data, neither the GLP-1R agonist liraglutide nor the DPP-4 inhibitor sitagliptin established a solid link with the increase of risk for pancreatic ductal adenocarcinoma in the therapy of patients with T2DM. Ongoing surveillance will be important in future outcome 
and epidemiologic studies that will be performed as the indications for use of these drugs expand; however, existing studies have been reassuring to date.

The effects of GLP-1R activation by liraglutide on human pancreatic cancer cells, MIA PaCa-2 and PANC1, were studied recently (Zhao et al., 2014). Liraglutide dose- and time-dependently increased GLP-1R expression, arrested cell cycles in the S phase, and induced apoptosis through stimulation of cAMP production and inhibition of Akt and ERK1/2 pathways. Pancreatic tumor growth was also attenuated by liraglutide in a mouse xenograft model in vivo. In patients with pancreatic cancer, $43.3 \%$ were GLP-1R-negative (13 of 30) and, in GLP-1R-positive tissues, tumor size was inversely correlated with GLP-1R expression. This is the only report of the cytoreductive effect of GLP-1R signaling activated by liraglutide on pancreatic cancer cells (Zhao et al., 2014).

Thyroid cancer is a relatively rare disease, although its incidence has increased at an alarming rate in both men and women in the United States (AschebrookKilfoy et al., 2011; Udelsman and Zhang, 2014). C cells constitute a minor fraction of the thyroid mass $(0.1 \%)$ and are the only cells that produce calcitonin (Huang et al., 2006). GLP-1R agonists are capable of inducing increased calcitonin gene expression and $\mathrm{C}$ cell hyperplasia in the thyroid of wild-type, but not the GLP-1R knockout ( $\left.G l p-1 r^{-1-}\right)$ mice (Yamada et al., 2008; Madsen et al., 2012). Long-term exposure to GLP-1R agonists resulted in $\mathrm{C}$ cell proliferation and the formation of $\mathrm{C}$ cell adenomas and (medullary thyroid) carcinomas in rodents (Elashoff et al., 2011; Bulchandani et al., 2012; Rosol, 2013). Data derived from the FDA Adverse Event Reporting System database also indicate a significantly elevated risk for thyroid cancer (histology not specified) with exenatide, but not sitagliptin treatment (Nauck and Friedrich, 2013). Several cases of thyroid cancer were reported during the liraglutide clinical development program (Neumiller et al., 2010; Elashoff et al., 2011). Thus, a Black Box warning regarding the risk of thyroid $\mathrm{C}$ cell cancer was labeled in all approved long-acting GLP-1 analogs (Parks and Rosebraugh, 2010; Andersen et al., 2013). However, a meta-analysis of serious adverse events reported with GLP-1R agonists indicates that neither liraglutide nor exenatide had an association with an increased risk of thyroid cancer (Alves et al., 2012). Such a discrepancy between humans and rodents may reside on the different expression level of GLP-1R in the thyroid because $\mathrm{C}$ cells are very sparse in primates when compared with rodents (Gallo, 2013; Pyke et al., 2014). Adequately powered long-term epidemiologic studies will be necessary to clarify the association between GLP-1-based therapies and the risk of thyroid cancer (Andersen et al., 2013).

Besides the pancreas and thyroid, GLP-1R is widely expressed in other tissues, such as the stomach, pituitary, heart, lung, kidney, and nervous system. Analysis of the FDA Adverse Event Reporting System database suggests that for several malignancies, excluding pancreatic and thyroid cancers, exenatide and sitagliptin apparently significantly reduced the odds ratios of some special cancers, such as lung cancer, prostate cancer, lymphoma/multiple myeloma for exenatide, and colon cancer and prostate cancer for sitagliptin (Vigneri et al., 2009; Koehler et al., 2011; Nauck and Friedrich, 2013). However, the data need to be interpreted with caution because the results are based on relatively small numbers of tumors and most likely are subject to reporting bias.

\section{E. GLP-1R Antagonists}

Under normal physiologic conditions, GLP-1 is secreted directly by the $\mathrm{L}$ cells of the gastrointestinal tract and, as described in this review, acts predominantly to enhance insulin secretion, decrease glucagon secretion, and inhibit gastric motility (II. Glucagon-Like Peptide-1). Based on these data, the main therapeutic strategy targeting GLP-1R has been development of agonists for the treatment of hyperglycemia.

It is established that the insulinotropic action of GLP-1 is highly glucose-dependent such that excessive GLP-1 secretion or sensitivity will not lead to hypoglycemia. However, clinical studies demonstrate that administration of GLP-1 in the presence of a nonglucosedependent insulin secretagogue (e.g., a sulphonylurea that acts on the $\mathrm{K}_{\mathrm{ATP}}$ channel) or even directly infusing supraphysiological levels of GLP-1 into normal subjects is associated with an increased risk of hypoglycemia (Toft-Nielsen et al., 1999; Buse et al., 2004). These data suggest that under rare conditions in which either the secretion of or sensitivity to GLP-1 is significantly enhanced, patients may display an increased incidence of insulin secretion and concomitant hypoglycemia. Furthermore, these conditions would be predicted to be responsive to treatment with a GLP-1R antagonist.

GLP-1R has a limited expression profile, and its agonists exhibit an excellent safety record, as indicated by the successful treatment of patients with T2DM. Furthermore, mouse GLP-1R knockouts are viable, develop normally, and demonstrate no overt phenotype (Scrocchi et al., 1996). Therefore, it is perhaps unsurprising that interest in the potential therapeutic opportunity for GLP$1 \mathrm{R}$ antagonists has emerged only recently, originating largely from a small number of reported clinical conditions that present severe hypoglycemia. These will be briefly discussed in the following paragraphs.

Congenital hyperinsulinism (CHI) represents the most frequent cause of severe, persistent hyperinsulinemic hypoglycemia in newborn babies and children, occurring in approximately $1 / 25,000$ to $1 / 50,000$ births (Lord et al., 2015). CHI is caused by genetic defects in key genes responsible for regulating insulin secretion and arises as a consequence of excess insulin secretion from the pancreatic $\beta$ cells (Rahman et al., 2015). Insulin directly lowers blood sugars, causing hypoglycemia, 
but insulin hypersecretion also reduces the supply of alternative sources of energy substrates for oxidative metabolism in the CNS. As these normally act as a protective measure against hypoglycemia, adverse neurodevelopment outcomes affect approximately onethird of all patients (Avatapalle et al., 2013). The most severe forms of $\mathrm{CHI}$ accounting for approximately $45 \%$ of cases are due to recessive inactivating mutations in ABCC8 and KCJN11 that encode the two components of the $\beta$ cell ATP-sensitive $\mathrm{K}^{+}$channel (SUR1 and Kir6.2, respectively). The current treatment paradigm principally involves agonists of $K_{\mathrm{ATP}}$ channels (diazoxide) and somatostatin receptors. However, as the pathology of CHI mainly involves genetic defects in $\mathrm{K}_{\mathrm{ATP}}$ channels, these patients $(>50 \%)$ will therefore be refractory to diazoxide treatment that acts via this receptor. Both targets are expressed on $\beta$ cells, but also many other cell types; and this widespread expression profile contributes to multiple off-target complications of these drugs. Pharmacologically unresponsive $\mathrm{CHI}$ requires surgical intervention that may be a limited pancreatectomy/ lesionectomy, or in some cases children will require a near-total pancreatectomy (95-98\%). Despite surgery, patients with the diffuse form of the disease often require further surgical episodes, and a significant majority of them will develop iatrogenic and early-onset diabetes.

The initial link between CHI and GLP-1 was first reported in rodent models of hyperinsulinism in which the $\mathrm{K}_{\mathrm{ATP}}$ channel subunit ABCC8 was knocked out (De Leon et al., 2008). In this study, treatment with the GLP-1R antagonist exendin ${ }_{9-39}$ decreased cAMP levels, insulin secretion, and glucose disposal. Interestingly, these effects were generated in the fasting state (i.e., when glucose levels, and hence GLP-1 levels, should have been lowest) and in isolated islets to observe impact on both the basal and stimulated insulin secretion. Recently, these findings have been extended to testing exendin ${ }_{9-39}$ in adult human subjects with $\mathrm{CHI}$ owing to inactivating mutations in the $\mathrm{K}_{\mathrm{ATP}}$ channel. Acute infusion of exendin ${ }_{9-39}$ significantly increased mean basal glucose levels and glucose area-under-thecurve, and markedly lowered the insulin:glucose ratios (Calabria et al., 2012). Currently, it is unclear in this setting whether the levels of GLP-1 are increased, or whether the responsiveness to exendin ${ }_{9-39}$ reflects the inverse agonist property of this peptide in controlling excessive insulin secretion by $\beta$ cells. Recent published data from the Dunne laboratory in Manchester support the identification of a specific subpopulation with elevated GLP-1 levels that may at least partly explain the pathophysiological role of GLP-1 in this condition.

Roux-en-Y gastric bypass surgery is being used increasingly in the treatment of morbidly obese type 2 diabetic patients, and in the vast majority of patients this results in a very beneficial outcome with significant weight loss and resolution of diabetes. However, in a small minority of patients, gastric bypass surgery can lead to a profound postprandial hyperinsulinemic hypoglycemic state that emerges several years after surgery (Patti et al., 2005; Ashrafian et al., 2011). Typically, adult patients experience dizziness, weakness, headache, confusion, lethargy, slurred speech, coma, and seizures depending on the severity and duration of the hypoglycemic episode. In a landmark paper (Marsk et al., 2010), the incidence of severe hyperinsulinemic hypoglycemia was highlighted in approximately $0.2-0.5 \%$ of 5040 patients undergoing gastric bypass surgery in Sweden between 1986 and 2006. Discussion with clinical trust representatives of United Kingdom National Health Service confirmed this as an increasingly recognized unmet need, with the figure requiring pharmacological therapy after bypass surgery in the United Kingdom likely to be closer to $1 \%$, in which by the end of 2012 a total of 18,577 procedures had been performed under the National Health Service. In the United States, the American Society for Metabolic and Bariatric Surgery reported the number of procedures increased from about 16,000 in the early 1990 s to more than 103,000 in 2003 , with the total number of surgeries performed by the end of 2005 exceeding 590,000. Based on such evidence, it is likely that this condition represents an area of considerable future commercial value that is predicted to increase in market size as the awareness and related diagnosis continue to expand.

A number of alternative hypotheses have been suggested to explain postprandial hyperinsulinemic hypoglycemia, as follows: 1) an exaggerated form of dumping syndrome; 2) the result of failure of the pancreas to revert after weight loss; and 3) an outcome from altered incretin secretion. Roux-en-Y gastric bypass surgery has been demonstrated to significantly increase the levels of GLP-1 secretion. Although the mechanism behind the postsurgery hyperinsulinemic hypoglycemia syndrome remains to be confirmed, an emerging number of affected patients present with higher insulin and GLP-1 responses (Service et al., 2005; Salehi et al., 2011). Furthermore, the effects of exogenously administered GLP-1 on gastrointestinal motility and secretion can be blocked by exendin ${ }_{9-39}$ (Schirra et al., 2006). Very recently, Salehi et al. (2014) have reported that this severe postprandial hypoglycemia in gastric bypass patients can be corrected by infusion of exendin ${ }_{9-39}$, consistent with a fundamental role for GLP-1 and its receptor in this mechanism.

Currently, no small-molecule GLP-1R antagonist is available that can be used to further understand the consequences of an overactive GLP-1 system. However, exendin $_{9-39}$ is a selective, competitive GLP-1R antagonist that blocks GLP-1-mediated insulin secretion in vitro and in vivo and impairs glucose tolerance in response to endogenous and exogenous GLP-1 in humans and a variety of rodent models (Schirra et al., 1998; Edwards et al., 1999). Moreover, a number of groups have reported that exendin $_{9-39}$ inhibits insulin secretion even in the absence of increased GLP-1 levels, suggesting that exendin ${ }_{9-39}$ may be an inverse agonist of GLP-1R (Serre et al., 1998; 
Salehi et al., 2011). A modified version of exendin $9-39$ has also been developed (Patterson et al., 2011) and was demonstrated to cause significant increases in glucose intolerance, food intake, and body weight in diet-induced obese mice, further supporting a role for endogenous GLP1 as a key hormone in the obese state.

Clearly, the findings discussed above have a number of important implications that form the scientific basis for targeting GLP-1R with specific small-molecule antagonists. These not only highlight possible control of hyperinsulinemic hypoglycemia in different patient populations, but also suggest that an antagonist may reduce the enhanced rate of $\beta$ cell expansion associated with $\mathrm{CHI}$, thereby offering potential disease-modifying treatment opportunities.

\section{Conclusions}

In this review, we have provided insights into the discovery, characterization, physiology, and pharmacology of GLP-1 and GLP-1R, a hormone-receptor system that seems to be ideally designed to facilitate the management of T2DM. This peptide hormone is secreted from enteroendocrine $\mathrm{L}$ cells in the distal intestine, exerting its effects through a class B GPCR on various target cells. Most prominent among these are the pancreatic islet $\beta$ cell, where GLP- 1 exerts a glucosedependent insulinotropic action. It also has other roles in glucose homoeostasis, as well as in regulating gastric motility and appetite useful in the control of body weight. A series of analogs of the GLP-1 peptide have been developed to enhance activity and bioavailability, but efforts in obtaining therapeutically viable nonpeptidic GLP-1R modulators were less successful. The molecular basis of the docking and action of these ligands is reviewed, along with implications for their spectrum of biologic actions. Some of these GLP-1R agonists have already launched to the worldwide market with many more under development. Such an endeavor will definitely lead to better and more efficacious drugs to treat T2DM and obesity.

\section{Acknowledgments}

We thank Dr. Xiao Luo for information collection.

\section{Authorship Contributions}

Participated in research design: Wang.

Wrote or contributed to the writing of the manuscript: de Graaf, Donnelly, Wootten, Lau, Sexton, Miller, Ahn, Liao, Fletcher, Yang, Brown, Zhou, Deng, Wang.

\section{References}

Abrahamsen N, Lundgren K, and Nishimura E (1995) Regulation of glucagon receptor mRNA in cultured primary rat hepatocytes by glucose and cAMP. $J$ Biol Chem 270:15853-15857.

Adelhorst K, Hedegaard BB, Knudsen LB, and Kirk O (1994) Structure-activity studies of glucagon-like peptide-1. J Biol Chem 269:6275-6278.

Ahn J-M, Murage EN, Lo S-T, Lin M, and Sun X (2011) Highly constrained GLP-1 analogues as non-invasive PET imaging agents for the assessment of pancreatic beta-cell mass, in Peptides: Building Bridges, Proceedings of the 22nd American Peptide Symposium (Lebl M ed); 2011 June 25-30; San Diego, CA. pp 220-221, American Peptide Society.
Al-Sabah S and Donnelly D (2003a) A model for receptor-peptide binding at the glucagon-like peptide-1 (GLP-1) receptor through the analysis of truncated ligands and receptors. $\mathrm{Br} J$ Pharmacol 140:339-346.

Al-Sabah S and Donnelly D (2003b) The positive charge at Lys-288 of the glucagonlike peptide-1 (GLP-1) receptor is important for binding the N-terminus of peptide agonists. FEBS Lett 553:342-346.

Alaña I, Parker JC, Gault VA, Flatt PR, O'Harte FP, Malthouse JP, and Hewage CM (2006) NMR and alanine scan studies of glucose-dependent insulinotropic polypeptide in water. J Biol Chem 281:16370-16376.

Albano JD, Ekins RP, Maritz G, and Turner RC (1972) A sensitive, precise radioimmunoassay of serum insulin relying on charcoal separation of bound and free hormone moieties. Acta Endocrinol 70:487-509.

Alken M, Rutz C, Köchl R, Donalies U, Oueslati M, Furkert J, Wietfeld D, Hermosilla R, Scholz A, Beyermann M, et al. (2005) The signal peptide of the rat corticotropinreleasing factor receptor 1 promotes receptor expression but is not essential for establishing a functional receptor. Biochem $J$ 390:455-464.

Alves C, Batel-Marques F, and Macedo AF (2012) A meta-analysis of serious adverse events reported with exenatide and liraglutide: acute pancreatitis and cancer Diabetes Res Clin Pract 98:271-284.

Andersen DK, Andren-Sandberg Å, Duell EJ, Goggins M, Korc M, Petersen GM, Smith JP, and Whitcomb DC (2013) Pancreatitis-diabetes-pancreatic cancer: summary of an NIDDK-NCI workshop. Pancreas 42:1227-1237.

Andersen L, Dinesen B, Jørgensen PN, Poulsen F, and Røder ME (1993) Enzyme immunoassay for intact human insulin in serum or plasma. Clin Chem 39:578-582

Angel TE, Chance MR, and Palczewski K (2009) Conserved waters mediate structural and functional activation of family A (rhodopsin-like) G protein-coupled receptors. Proc Natl Acad Sci USA 106:8555-8560.

Aschebrook-Kilfoy B, Ward MH, Sabra MM, and Devesa SS (2011) Thyroid cancer incidence patterns in the United States by histologic type, 1992-2006. Thyroid 21: 125-134

Ashrafian H, Athanasiou T, Li JV, Bueter M, Ahmed K, Nagpal K, Holmes E, Darzi A, and Bloom SR (2011) Diabetes resolution and hyperinsulinaemia after metabolic Roux-en-Y gastric bypass. Obes Rev 12:e257-e272.

Astrup A, Rössner S, Van Gaal L, Rissanen A, Niskanen L, Al Hakim M, Madsen J, Rasmussen MF, and Lean ME; NN8022-1807 Study Group (2009) Effects of liraglutide in the treatment of obesity: a randomised, double-blind, placebo-controlled study. Lancet 374:1606-1616.

Audigier Y, Friedlander M, and Blobel G (1987) Multiple topogenic sequences in bovine opsin. Proc Natl Acad Sci USA 84:5783-5787.

Avatapalle HB, Banerjee I, Shah S, Pryce M, Nicholson J, Rigby L, Caine L, Didi M, Skae M, Ehtisham S, et al. (2013) Abnormal neurodevelopmental outcomes are common in children with transient congenital hyperinsulinism. Front Endocrinol 4:60

Aviles-Olmos I, Dickson J, Kefalopoulou Z, Djamshidian A, Ell P, Soderlund T, Whitton P, Wyse R, Isaacs T, Lees A, et al. (2013) Exenatide and the treatment of patients with Parkinson's disease. J Clin Invest 123:2730-2736.

Avogaro A, Vigili de Kreutzenberg S, and Fadini GP (2014) Cardiovascular actions of GLP-1 and incretin-based pharmacotherapy. Curr Diab Rep 14:483.

Baggio LL and Drucker DJ (2007) Biology of incretins: GLP-1 and GIP. Gastroenterology 132:2131-2157.

Baggio LL, Kim JG, and Drucker DJ (2004) Chronic exposure to GLP-1R agonists promotes homologous GLP-1 receptor desensitization in vitro but does not attenuate GLP-1R-dependent glucose homeostasis in vivo. Diabetes 53 (Suppl 3): S205-S214.

Ballesteros JA and Weinstein H (1995) Integrated methods for the construction of three-dimensional models and computational probing of structure-function relations in G protein-coupled receptors. Methods Neurosci 25:366-428.

Ban K, Kim KH, Cho CK, Sauvé M, Diamandis EP, Backx PH, Drucker DJ, and Husain M (2010) Glucagon-like peptide (GLP)-1(9-36)amide-mediated cytoprotection is blocked by exendin(9-39) yet does not require the known GLP-1 receptor. Endocrinology 151:1520-1531.

Barragán JM, Rodríguez RE, Eng J, and Blázquez E (1996) Interactions of exendin(9-39) with the effects of glucagon-like peptide-1-(7-36) amide and of exendin-4 on arterial blood pressure and heart rate in rats. Regul Pept 67:63-68.

Barrera JG, Sandoval DA, D'Alessio DA, and Seeley RJ (2011) GLP-1 and energy balance: an integrated model of short-term and long-term control. Nat Rev Endocrinol 7:507-516.

Bassil F, Fernagut PO, Bezard E, and Meissner WG (2014) Insulin, IGF-1 and GLP-1 signaling in neurodegenerative disorders: targets for disease modification? Prog Neurobiol 118:1-18.

Bazarsuren A, Grauschopf U, Wozny M, Reusch D, Hoffmann E, Schaefer W, Panzner $\mathrm{S}$, and Rudolph R (2002) In vitro folding, functional characterization, and disulfide pattern of the extracellular domain of human GLP-1 receptor. Biophys Chem 96: 305-318.

Beinborn M, Worrall CI, McBride EW, and Kopin AS (2005) A human glucagon-like peptide-1 receptor polymorphism results in reduced agonist responsiveness. Regul Pept 130:1-6.

Belin D, Bost S, Vassalli JD, and Strub K (1996) A two-step recognition of signal sequences determines the translocation efficiency of proteins. $E M B O J \mathbf{1 5}$ $468-478$.

Bell GI, Sanchez-Pescador R, Laybourn PJ, and Najarian RC (1983a) Exon duplication and divergence in the human preproglucagon gene. Nature 304:368-371.

Bell GI, Santerre RF, and Mullenbach GT (1983b) Hamster preproglucagon contain the sequence of glucagon and two related peptides. Nature 302:716-718.

Bendtsen JD, Nielsen H, von Heijne G, and Brunak S (2004) Improved prediction of signal peptides: SignalP 3.0. J Mol Biol 340:783-795.

Bergwitz C, Gardella TJ, Flannery MR, Potts JT Jr, Kronenberg HM, Goldring SR, and Jüppner H (1996) Full activation of chimeric receptors by hybrids between parathyroid hormone and calcitonin: evidence for a common pattern of ligandreceptor interaction. J Biol Chem 271:26469-26472. 
Bhashyam S, Fields AV, Patterson B, Testani JM, Chen L, Shen YT, and Shannon RP (2010) Glucagon-like peptide-1 increases myocardial glucose uptake via p38alpha MAP kinase-mediated, nitric oxide-dependent mechanisms in conscious dogs with dilated cardiomyopathy. Circ Heart Fail 3:512-521.

Bode HP, Moormann B, Dabew R, and Göke B (1999) Glucagon-like peptide 1 elevates cytosolic calcium in pancreatic beta-cells independently of protein kinase A. Endocrinology 140:3919-3927.

Bose AK, Mocanu MM, Carr RD, Brand CL, and Yellon DM (2005a) Glucagon-like peptide 1 can directly protect the heart against ischemia/reperfusion injury. $\mathrm{Di}$ abetes 54:146-151.

Bose AK, Mocanu MM, Carr RD, and Yellon DM (2005b) Glucagon like peptide-1 is protective against myocardial ischemia/reperfusion injury when given either as a preconditioning mimetic or at reperfusion in an isolated rat heart model. Cardiovasc Drugs Ther 19:9-11.

Bose AK, Mocanu MM, Carr RD, and Yellon DM (2007) Myocardial ischaemiareperfusion injury is attenuated by intact glucagon like peptide-1 (GLP-1) in the in vitro rat heart and may involve the p70s6K pathway. Cardiovasc Drugs Ther 21 253-256

Braun W, Wider G, Lee KH, and Wüthrich K (1983) Conformation of glucagon in a lipid-water interphase by $1 \mathrm{H}$ nuclear magnetic resonance. J Mol Biol 169:921-948.

Brom M, Oyen WJ, Joosten L, Gotthardt M, and Boerman OC (2010) 68Ga-labelled exendin-3, a new agent for the detection of insulinomas with PET. Eur J Nucl Med Mol Imaging 37:1345-1355.

Brown JC, Dryburgh JR, Ross SA, and Dupré J (1975) Identification and actions of gastric inhibitory polypeptide. Recent Prog Horm Res 31:487-532.

Bulchandani D, Nachnani JS, Herndon B, Molteni A, Pathan MH, Quinn T, Hamdan HA, Alba LM, and Graves L (2012) Effect of exendin (exenatide): GLP 1 receptor agonist on the thyroid and parathyroid gland in a rat model. Eur J Pharmacol $\mathbf{6 9 1}$ $292-296$

Bullock BP, Heller RS, and Habener JF (1996) Tissue distribution of messenger ribonucleic acid encoding the rat glucagon-like peptide-1 receptor. Endocrinology 137:2968-2978.

Burcelin R, Da Costa A, Drucker D, and Thorens B (2001) Glucose competence of the hepatoportal vein sensor requires the presence of an activated glucagon-like peptide-1 receptor. Diabetes 50:1720-1728.

Burcelin R, Dolci W, and Thorens B (1999) Long-lasting antidiabetic effect of a dipeptidyl peptidase IV-resistant analog of glucagon-like peptide-1. Metabolism 48 252-258.

Buse JB, Bergenstal RM, Glass LC, Heilmann CR, Lewis MS, Kwan AY, Hoogwerf BJ, and Rosenstock J (2011) Use of twice-daily exenatide in Basal insulin-treated patients with type 2 diabetes: a randomized, controlled trial. Ann Intern Med 154 103-112.

Buse JB, Henry RR, Han J, Kim DD, Fineman MS, and Baron AD; Exenatide-113 Clinical Study Group (2004) Effects of exenatide (exendin-4) on glycemic control over 30 weeks in sulfonylurea-treated patients with type 2 diabetes. Diabetes Care 27:2628-2635

Buteau J (2011) GLP-1 signaling and the regulation of pancreatic $\beta$-cells mass/ function. Avances en Diabetología 27:3-8.

Buteau J, El-Assaad W, Rhodes CJ, Rosenberg L, Joly E, and Prentki M (2004) Glucagon-like peptide-1 prevents beta cell glucolipotoxicity. Diabetologia 47:806-815.

Buteau J, Spatz ML, and Accili D (2006) Transcription factor FoxO1 mediates glucagon-like peptide-1 effects on pancreatic beta-cell mass. Diabetes 55:1190-1196

Calabria AC, Li C, Gallagher PR, Stanley CA, and De León DD (2012) GLP-1 receptor antagonist exendin-(9-39) elevates fasting blood glucose levels in congenital hyperinsulinism owing to inactivating mutations in the ATP-sensitive $\mathrm{K}^{+}$channel. Diabetes 61:2585-2591.

Campbell JE and Drucker DJ (2013) Pharmacology, physiology, and mechanisms of incretin hormone action. Cell Metab 17:819-837.

Campos RV, Lee YC, and Drucker DJ (1994) Divergent tissue-specific and developmental expression of receptors for glucagon and glucagon-like peptide- 1 in the mouse. Endocrinology 134:2156-2164.

Carraro-Lacroix LR, Malnic G, and Girardi AC (2009) Regulation of Na+/H+ exchanger NHE3 by glucagon-like peptide 1 receptor agonist exendin- 4 in renal proximal tubule cells. Am J Physiol Renal Physiol 297:F1647-F1655.

Challa TD, Beaton N, Arnold M, Rudofsky G, Langhans W, and Wolfrum C (2012) Regulation of adipocyte formation by GLP-1/GLP-1R signaling. J Biol Chem 287: $6421-6430$

Chen D, Liao J, Li N, Zhou C, Liu Q, Wang G, Zhang R, Zhang S, Lin L, Chen K, et al. (2007) A nonpeptidic agonist of glucagon-like peptide 1 receptors with efficacy in diabetic db/db mice. Proc Natl Acad Sci USA 104:943-948.

Chen Q, Miller LJ, and Dong M (2010a) Role of N-linked glycosylation in biosynthesis, trafficking, and function of the human glucagon-like peptide 1 receptor Am J Physiol Endocrinol Metab 299:E62-E68.

Chen Q, Pinon DI, Miller LJ, and Dong M (2009) Molecular basis of glucagon-like peptide 1 docking to its intact receptor studied with carboxyl-terminal photolabile probes. J Biol Chem 284:34135-34144.

Chen Q, Pinon DI, Miller LJ, and Dong M (2010b) Spatial approximations between residues 6 and 12 in the amino-terminal region of glucagon-like peptide 1 and its receptor: a region critical for biological activity. J Biol Chem 285:24508-24518.

Cheong YH, Kim MK, Son MH, and Kaang BK (2012) Two small molecule agonists of glucagon-like peptide-1 receptor modulate the receptor activation response differently. Biochem Biophys Res Commun 417:558-563.

Chepurny OG, Hussain MA, and Holz GG (2002) Exendin-4 as a stimulator of rat insulin I gene promoter activity via bZIP/CRE interactions sensitive to serine/ threonine protein kinase inhibitor Ro 31-8220. Endocrinology 143:2303-2313.

Chowdhry V and Westheimer FH (1979) Photoaffinity labeling of biological systems. Annu Rev Biochem 48:293-325.

Christensen M, Miossec P, Larsen BD, Werner U, and Knop FK (2014) The design and discovery of lixisenatide for the treatment of type 2 diabetes mellitus. Expert Opin Drug Discov 9:1223-1251.
Claeysen S, Cochet M, Donneger R, Dumuis A, Bockaert J, and Giannoni P (2012) Alzheimer culprits: cellular crossroads and interplay. Cell Signal 24:1831-1840.

Clérico EM, Maki JL, and Gierasch LM (2008) Use of synthetic signal sequences to explore the protein export machinery. Biopolymers 90:307-319.

Connolly BM, Vanko A, McQuade P, Guenther I, Meng X, Rubins D, Waterhouse R, Hargreaves R, Sur C, and Hostetler E (2012) Ex vivo imaging of pancreatic beta cells using a radiolabeled GLP-1 receptor agonist. Mol Imaging Biol 14: $79-87$

Coopman K, Huang Y, Johnston N, Bradley SJ, Wilkinson GF, and Willars GB (2010 Comparative effects of the endogenous agonist glucagon-like peptide-1 (GLP-1)-(736) amide and the small-molecule ago-allosteric agent "compound 2" at the GLP-1 receptor. J Pharmacol Exp Ther 334:795-808

Coopman K, Wallis R, Robb G, Brown AJ, Wilkinson GF, Timms D, and Willars GB (2011) Residues within the transmembrane domain of the glucagon-like peptide-1 receptor involved in ligand binding and receptor activation: modelling the ligandbound receptor. Mol Endocrinol 25:1804-1818.

Crajoinas RO, Oricchio FT, Pessoa TD, Pacheco BP, Lessa LM, Malnic G, and Girardi AC (2011) Mechanisms mediating the diuretic and natriuretic actions of the incretin hormone glucagon-like peptide-1. Am $J$ Physiol Renal Physiol 301: F355-F363.

Creutzfeldt W (1979) The incretin concept today. Diabetologia 16:75-85

Creutzfeldt W (2005) The [pre-] history of the incretin concept. Regul Pept 128:87-91.

Creutzfeldt W and Ebert R (1985) New developments in the incretin concept. Diabetologia 28:565-573.

Creutzfeldt WO, Kleine N, Willms B, Orskov C, Holst JJ, and Nauck MA (1996) Glucagonostatic actions and reduction of fasting hyperglycemia by exogenous glucagon-like peptide I(7-36) amide in type I diabetic patients. Diabetes Care 19: $580-586$

Cullinan CA, Brady EJ, Saperstein R, and Leibowitz MD (1994) Glucose-dependent alterations of intracellular free calcium by glucagon-like peptide-1(7-36amide) in individual ob/ob mouse beta-cells. Cell Calcium 15:391-400.

Curran AR and Engelman DM (2003) Sequence motifs, polar interactions and conformational changes in helical membrane proteins. Curr Opin Struct Biol 13: $412-417$.

Cvetković RS and Plosker GL (2007) Exenatide: a review of its use in patients with type 2 diabetes mellitus (as an adjunct to metformin and/or a sulfonylurea). Drugs 67:935-954.

D’Amico E, Hui H, Khoury N, Di Mario U, and Perfetti R (2005) Pancreatic beta-cells expressing GLP-1 are resistant to the toxic effects of immunosuppressive drugs. $J$ Mol Endocrinol 34:377-390.

Davis HR Jr, Mullins DE, Pines JM, Hoos LM, France CF, Compton DS, Graziano MP, Sybertz EJ, Strader CD, and Van Heek M (1998) Effect of chronic central administration of glucagon-like peptide-1 (7-36) amide on food consumption and body weight in normal and obese rats. Obes Res 6:147-156.

Day JW, Li P, Patterson JT, Chabenne J, Chabenne MD, Gelfanov VM, and Dimarch RD (2011) Charge inversion at position 68 of the glucagon and glucagon-like peptide-1 receptors supports selectivity in hormone action. J Pept Sci 17:218-225.

de Graaf C, Rein C, Piwnica D, Giordanetto F, and Rognan D (2011) Structure-based discovery of allosteric modulators of two related class B G-protein-coupled receptors. ChemMedChem 6:2159-2169.

de Heer J, Rasmussen C, Coy DH, and Holst JJ (2008) Glucagon-like peptide-1, but not glucose-dependent insulinotropic peptide, inhibits glucagon secretion via somatostatin (receptor subtype 2) in the perfused rat pancreas. Diabetologia 51: $2263-2270$

De León DD, Li C, Delson MI, Matschinsky FM, Stanley CA, and Stoffers DA (2008) Exendin-(9-39) corrects fasting hypoglycemia in SUR-1-/- mice by lowering cAMP in pancreatic beta-cells and inhibiting insulin secretion. J Biol Chem 283 25786-25793.

de Wit HM, Vervoort GMM, Jansen HJ, de Grauw WJC, de Galan BE, and Tack CJ (2014) Liraglutide reverses pronounced insulin-associated weight gain, improves glycaemic control and decreases insulin dose in patients with type 2 diabetes: a 26 week, randomised clinical trial (ELEGANT). Diabetologia 57:1812-1819.

Deacon CF and Holst JJ (2009) Immunoassays for the incretin hormones GIP and GLP-1. Best Pract Res Clin Endocrinol Metab 23:425-432.

Deacon CF, Johnsen AH, and Holst JJ (1995a) Degradation of glucagon-like peptide1 by human plasma in vitro yields an N-terminally truncated peptide that is a major endogenous metabolite in vivo. J Clin Endocrinol Metab 80:952-957.

Deacon CF, Knudsen LB, Madsen K, Wiberg FC, Jacobsen O, and Holst JJ (1998) Dipeptidyl peptidase IV resistant analogues of glucagon-like peptide-1 which have extended metabolic stability and improved biological activity. Diabetologia 41: 271-278.

Deacon CF, Nauck MA, Toft-Nielsen M, Pridal L, Willms B, and Holst JJ (1995b) Both subcutaneously and intravenously administered glucagon-like peptide I are rapidly degraded from the $\mathrm{NH} 2$-terminus in type II diabetic patients and in healthy subjects. Diabetes 44:1126-1131.

Denker PS and Dimarco PE (2006) Exenatide (exendin-4)-induced pancreatitis: a case report. Diabetes Care 29:471.

Dharmalingam M, Sriram U, and Baruah MP (2011) Liraglutide: a review of its therapeutic use as a once daily GLP-1 analog for the management of type 2 diabetes mellitus. Indian J Endocrinol Metab 15:9-17.

Diamant M, Van Gaal L, Guerci B, Stranks S, Han J, Malloy J, Boardman MK, and Trautmann ME (2014) Exenatide once weekly versus insulin glargine for type 2 diabetes (DURATION-3): 3-year results of an open-label randomised trial. Lancet Diabetes Endocrinol 2:464-473.

Dillon JS, Tanizawa Y, Wheeler MB, Leng XH, Ligon BB, Rabin DU, Yoo-Warren H, Permutt MA, and Boyd AE 3rd (1993) Cloning and functional expression of the human glucagon-like peptide-1 (GLP-1) receptor. Endocrinology 133:1907-1910.

Dods RL and Donnelly D (2015) The peptide agonist-binding site of the glucagon-like peptide-1 (GLP-1) receptor based on site-directed mutagenesis and knowledgebased modelling. Biosci Rep 36:e0285. 
Dong M, Pinon DI, and Miller LJ (2012) Site of action of a pentapeptide agonist at the glucagon-like peptide-1 receptor: insight into a small molecule agonist-binding pocket. Bioorg Med Chem Lett 22:638-641.

Dong JZ, Shen Y, Zhang J, Tsomaia N, Mierke DF, and Taylor JE (2011) Discovery and characterization of taspoglutide, a novel analogue of human glucagon-like peptide-1, engineered for sustained therapeutic activity in type 2 diabetes. $\mathrm{Di}$ abetes Obes Metab 13:19-25.

Donnelly D (2012) The structure and function of the glucagon-like peptide-1 receptor and its ligands. $\mathrm{Br} J$ Pharmacol 166:27-41.

Drucker DJ and Asa S (1988) Glucagon gene expression in vertebrate brain. J Biol Chem 263:13475-13478.

Drucker DJ, Dritselis A, and Kirkpatrick P (2010) Liraglutide. Nat Rev Drug Discov 9:267-268.

Drucker DJ, Philippe J, Mojsov S, Chick WL, and Habener JF (1987) Glucagon-like peptide I stimulates insulin gene expression and increases cyclic AMP levels in a rat islet cell line. Proc Natl Acad Sci USA 84:3434-3438.

Dungan KM, Povedano ST, Forst T, González JG, Atisso C, Sealls W, and Fahrbach JL (2014) Once-weekly dulaglutide versus once-daily liraglutide in metformintreated patients with type 2 diabetes (AWARD-6): a randomised, open-label, phase 3, non-inferiority trial. Lancet 384:1349-1357.

Dunphy JL, Taylor RG, and Fuller PJ (1998) Tissue distribution of rat glucagon receptor and GLP-1 receptor gene expression. Mol Cell Endocrinol 141:179-186.

Dupre J, Caussignac Y, McDonald TJ, and Van Vliet S (1991) Stimulation of glucagon secretion by gastric inhibitory polypeptide in patients with hepatic cirrhosis and hyperglucagonemia. J Clin Endocrinol Metab 72:125-129.

Dupre J, Ross SA, Watson D, and Brown JC (1973) Stimulation of insulin secretion by gastric inhibitory polypeptide in man. J Clin Endocrinol Metab 37:826-828.

During MJ, Cao L, Zuzga DS, Francis JS, Fitzsimons HL, Jiao X, Bland RJ, Klugmann M, Banks WA, Drucker DJ, et al. (2003) Glucagon-like peptide-1 receptor is involved in learning and neuroprotection. Nat Med 9:1173-1179.

Dyachok O and Gylfe E (2004) $\mathrm{Ca}\left({ }^{2+}\right)$-induced $\mathrm{Ca}\left({ }^{2+}\right)$ release via inositol 1,4,5trisphosphate receptors is amplified by protein kinase $\mathrm{A}$ and triggers exocytosis in pancreatic beta-cells. J Biol Chem 279:45455-45461.

Dyachok O, Isakov Y, Sågetorp J, and Tengholm A (2006) Oscillations of cyclic AMP in hormone-stimulated insulin-secreting beta-cells. Nature 439:349-352.

Dzhura I, Chepurny OG, Leech CA, Roe MW, Dzhura E, Xu X, Lu Y, Schwede F, Genieser HG, Smrcka AV, et al. (2011) Phospholipase C- $\varepsilon$ links Epac2 activation to the potentiation of glucose-stimulated insulin secretion from mouse islets of Langerhans. Islets 3:121-128.

Ebert R and Creutzfeldt W (1982) Influence of gastric inhibitory polypeptide antiserum on glucose-induced insulin secretion in rats. Endocrinology 111:1601-1606.

Ebert R and Creutzfeldt W (1987) Gastrointestinal peptides and insulin secretion. Diabetes Metab Rev 3:1-26.

Edvell A and Lindström P (1999) Initiation of increased pancreatic islet growth in young normoglycemic mice (Umeå +/?). Endocrinology 140:778-783.

Edwards CM, Todd JF, Mahmoudi M, Wang Z, Wang RM, Ghatei MA, and Bloom SR (1999) Glucagon-like peptide 1 has a physiological role in the control of postprandial glucose in humans: studies with the antagonist exendin 9-39. Diabetes 48: 86-93.

Einhorn L and Krapfenbauer K (2015) HTRF: a technology tailored for biomarker determination-novel analytical detection system suitable for detection of specific autoimmune antibodies as biomarkers in nanogram level in different body fluids. EPMA J 6:23.

Elahi D, Andersen DK, Brown JC, Debas HT, Hershcopf RJ, Raizes GS, Tobin JD, and Andres R (1979) Pancreatic alpha- and beta-cell responses to GIP infusion in normal man. Am J Physiol 237:E185-E191.

Elahi D, McAloon-Dyke M, Fukagawa NK, Meneilly GS, Sclater AL, Minaker KL, Habener JF, and Andersen DK (1994) The insulinotropic actions of glucosedependent insulinotropic polypeptide (GIP) and glucagon-like peptide-1 (7-37) in normal and diabetic subjects. Regul Pept 51:63-74.

Elashoff M, Matveyenko AV, Gier B, Elashoff R, and Butler PC (2011) Pancreatitis, pancreatic, and thyroid cancer with glucagon-like peptide-1-based therapies. Gastroenterology 141:150-156.

Elrick H, Stimmler L, Hlad CJ Jr, and Arai Y (1964) Plasma insulin response to oral and intravenous glucose administration. J Clin Endocrinol Metab 24:1076-1082.

Eng C, Kramer CK, Zinman B, and Retnakaran R (2014) Glucagon-like peptide-1 receptor agonist and basal insulin combination treatment for the management of type 2 diabetes: a systematic review and meta-analysis. Lancet 384:2228-2234.

Eng H, Sharma R, McDonald TS, Edmonds DJ, Fortin JP, Li X, Stevens BD, Griffith DA, Limberakis C, Nolte WM, et al. (2013) Demonstration of the innate electrophilicity of 4-(3-(benzyloxy)phenyl)-2-(ethylsulfinyl)-6-(trifluoromethyl)pyrimidine (BETP), a small-molecule positive allosteric modulator of the glucagon-like peptide-1 receptor. Drug Metab Dispos 41:1470-1479.

Erdogdu O, Nathanson D, Sjöholm A, Nyström T, and Zhang Q (2010) Exendin-4 stimulates proliferation of human coronary artery endothelial cells through eNOS-, PKA- and PI3K/Akt-dependent pathways and requires GLP-1 receptor. Mol Cell Endocrinol 325:26-35.

Esposito K, Chiodini P, Colao A, Lenzi A, and Giugliano D (2012) Metabolic syndrome and risk of cancer: a systematic review and meta-analysis. Diabetes Care $\mathbf{3 5}$ $2402-2411$

Fan H, Gong N, Li TF, Ma AN, Wu XY, Wang MW, and Wang YX (2015) The nonpeptide GLP-1 receptor agonist WB4-24 blocks inflammatory nociception by stimulating $\beta$-endorphin release from spinal microglia. Br J Pharmacol 172:64-79.

Farmer RW, Harrington CA, and Brown DH (1975) A simple radioimmunoassay for $3^{\prime}, 5^{\prime}$, cyclic adenosine monophosphate. Anal Biochem 64:455-460.

Fehmann HC and Habener JF (1991) Homologous desensitization of the insulinotropic glucagon-like peptide-I (7-37) receptor on insulinoma (HIT-T15) cells. Endocrinology 128:2880-2888.

Ferguson SS (2001) Evolving concepts in G protein-coupled receptor endocytosis: the role in receptor desensitization and signaling. Pharmacol Rev 53:1-24.
Flint A, Raben A, Astrup A, and Holst JJ (1998) Glucagon-like peptide 1 promotes satiety and suppresses energy intake in humans. $J$ Clin Invest 101:515-520.

Fortin JP, Schroeder JC, Zhu Y, Beinborn M, and Kopin AS (2010) Pharmacological characterization of human incretin receptor missense variants. $J$ Pharmacol Exp Ther 332:274-280.

Fox CB, Wayment JR, Myers GA, Endicott SK, and Harris JM (2009) Single-molecule fluorescence imaging of peptide binding to supported lipid bilayers. Anal Chem 81: $5130-5138$

Friedrichsen BN, Neubauer N, Lee YC, Gram VK, Blume N, Petersen JS, Nielsen JH, and Møldrup A (2006) Stimulation of pancreatic beta-cell replication by incretins involves transcriptional induction of cyclin D1 via multiple signalling pathways. $J$ Endocrinol 188:481-492.

Fritsche A, Stefan N, Hardt E, Häring H, and Stumvoll M (2000) Characterisation of beta-cell dysfunction of impaired glucose tolerance: evidence for impairment of incretin-induced insulin secretion. Diabetologia 43:852-858.

Fujimoto K, Shibasaki T, Yokoi N, Kashima Y, Matsumoto M, Sasaki T, Tajima N, Iwanaga T, and Seino S (2002) Piccolo, a Ca2+ sensor in pancreatic beta-cells: involvement of cAMP-GEFII.Rim2. Piccolo complex in cAMP-dependent exocytosis. $J$ Biol Chem 277:50497-50502.

Gallo M (2013) Thyroid safety in patients treated with liraglutide. $J$ Endocrinol Invest 36:140-145.

Gallwitz B, Ropeter T, Morys-Wortmann C, Mentlein R, Siegel EG, and Schmidt WE (2000) GLP-1-analogues resistant to degradation by dipeptidyl-peptidase IV in vitro. Regul Pept 86:103-111.

Gallwitz B, Witt M, Paetzold G, Morys-Wortmann C, Zimmermann B, Eckart K, Fölsch UR, and Schmidt WE (1994) Structure/activity characterization of glucagonlike peptide-1. Eur J Biochem 225:1151-1156.

Gao F, Harikumar KG, Dong M, Lam PC, Sexton PM, Christopoulos A, Bordner A, Abagyan R, and Miller LJ (2009) Functional importance of a structurally distinct homodimeric complex of the family B G protein-coupled secretin receptor. Mol Pharmacol 76:264-274.

Gao H, Niu G, Yang M, Quan Q, Ma Y, Murage EN, Ahn J-M, Kiesewetter DO, and Chen X (2011) PET of insulinoma using ${ }^{18} \mathrm{~F}-\mathrm{FBEM}-\mathrm{EM} 3106 \mathrm{~B}$, a new GLP-1 analogue. Mol Pharm 8:1775-1782.

Garber AJ (2012) Novel GLP-1 receptor agonists for diabetes. Expert Opin Investig Drugs 21:45-57.

Gault VA and Hölscher C (2008) GLP-1 agonists facilitate hippocampal LTP and reverse the impairment of LTP induced by beta-amyloid. Eur $J$ Pharmacol 587: 112-117.

Ge Y, Yang D, Dai A, Zhou C, Zhu Y, and Wang MW (2014) The putative signal peptide of glucagon-like peptide-1 receptor is not required for receptor synthesis but promotes receptor expression. Biosci Rep 34:e00152.

Gejl M, Starup-Linde J, Scheel-Thomsen J, Gregersen S, and Vestergaard P (2015) Risk of cardiovascular disease: the effects of diabetes and anti-diabetic drugs: a nested case-control study. Int J Cardiol 178:292-296.

Gengler S, McClean PL, McCurtin R, Gault VA, and Hölscher C (2012) Val(8)GLP-1 rescues synaptic plasticity and reduces dense core plaques in APP/PS1 mice. Neurobiol Aging 33:265-276.

Gentry PR, Sexton PM, and Christopoulos A (2015) Novel allosteric modulators of G protein-coupled receptors. J Biol Chem 290:19478-19488.

Gesellchen F, Prinz A, Zimmermann B, and Herberg FW (2006) Quantification of cAMP antagonist action in vitro and in living cells. Eur J Cell Biol 85:663-672.

Glaesner W, Vick AM, Millican R, Ellis B, Tschang SH, Tian Y, Bokvist K, Brenner M, Koester A, Porksen N, et al. (2010) Engineering and characterization of the long-acting glucagon-like peptide-1 analogue LY2189265, an Fc fusion protein. Diabetes Metab Res Rev 26:287-296.

Godoy-Matos AF (2014) The role of glucagon on type 2 diabetes at a glance. Diabetol Metab Syndr 6:91.

Göke R, Fehmann HC, Linn T, Schmidt H, Krause M, Eng J, and Göke B (1993a) Exendin-4 is a high potency agonist and truncated exendin-(9-39)-amide an antagonist at the glucagon-like peptide 1-(7-36)-amide receptor of insulin-secreting beta-cells. J Biol Chem 268:19650-19655.

Göke R, Just R, Lankat-Buttgereit B, and Göke B (1994) Glycosylation of the GLP-1 receptor is a prerequisite for regular receptor function. Peptides 15:675-681.

Göke R, Wagner B, Fehmann HC, and Göke B (1993b) Glucose-dependency of the insulin stimulatory effect of glucagon-like peptide-1 (7-36) amide on the rat pancreas. Res Exp Med 193:97-103.

Goland R, Freeby M, Parsey R, Saisho Y, Kumar D, Simpson N, Hirsch J, Prince M, Maffei A, Mann JJ, et al. (2009) 11C-dihydrotetrabenazine PET of the pancreas in subjects with long-standing type 1 diabetes and in healthy controls. J Nucl Med 50: 382-389.

Gong N, Fan H, Ma AN, Xiao Q, and Wang YX (2014a) Geniposide and its iridoid analogs exhibit antinociception by acting at the spinal GLP-1 receptors. Neuropharmacology 84:31-45.

Gong N, Ma AN, Zhang LJ, Luo XS, Zhang YH, Xu M, and Wang YX (2011) Sitespecific PEGylation of exenatide analogues markedly improved their glucoregulatory activity. Br J Pharmacol 163:399-412.

Gong N, Xiao Q, Zhu B, Zhang CY, Wang YC, Fan H, Ma AN, and Wang YX (2014b) Activation of spinal glucagon-like peptide-1 receptors specifically suppresses pain hypersensitivity. J Neurosci 34:5322-5334.

Goodman RH, Jacobs JW, Chin WW, Lund PK, Dee PC, and Habener JF (1980a) Nucleotide sequence of a cloned structural gene coding for a precursor of pancreatic somatostatin. Proc Natl Acad Sci USA 77:5869-5873.

Goodman RH, Lund PK, Jacobs JW, and Habener JF (1980b) Pre-prosomatostatins: products of cell-free translations of messenger RNAs from anglerfish islets. J Biol Chem 255:6549-6552.

Gotthardt M, Lalyko G, van Eerd-Vismale J, Keil B, Schurrat T, Hower M, Laverman P, Behr TM, Boerman OC, Göke B, et al. (2006) A new technique for in vivo imaging of specific GLP-1 binding sites: first results in small rodents. Regul Pept 137: 162-167. 
Gough SCL, Bode B, Woo V, Rodbard HW, Linjawi S, Poulsen P, Damgaard LH, and Buse JB; NN9068-3697 (DUAL-I) Trial Investigators (2014) Efficacy and safety of a fixed-ratio combination of insulin degludec and liraglutide (IDegLira) compared with its components given alone: results of a phase 3, open-label, randomised, 26-week, treat-to-target trial in insulin-naive patients with type 2 diabetes. Lancet Diabetes Endocrinol 2:885-893.

Graziano MP, Hey PJ, Borkowski D, Chicchi GG, and Strader CD (1993) Cloning and functional expression of a human glucagon-like peptide-1 receptor. Biochem Biophys Res Commun 196:141-146.

Green BD, Gault VA, Irwin N, Mooney MH, Bailey CJ, Harriott P, Greer B, Flatt PR, and O'Harte FP (2003) Metabolic stability, receptor binding, cAMP generation, insulin secretion and antihyperglycaemic activity of novel N-terminal Glu9substituted analogues of glucagon-like peptide-1. Biol Chem 384:1543-1551.

Green BD, Mooney MH, Gault VA, Irwin N, Bailey CJ, Harriott P, Greer B, Flatt PR and O'Harte FP (2004) Lys9 for Glu9 substitution in glucagon-like peptide-1(736)amide confers dipeptidylpeptidase IV resistance with cellular and metabolic actions similar to those of established antagonists glucagon-like peptide-1(936)amide and exendin (9-39). Metabolism 53:252-259.

Grieve DJ, Cassidy RS, and Green BD (2009) Emerging cardiovascular actions of the incretin hormone glucagon-like peptide-1: potential therapeutic benefits beyond glycaemic control? Br J Pharmacol 157:1340-1351.

Gromada J, Brock B, Schmitz O, and Rorsman P (2004) Glucagon-like peptide-1: regulation of insulin secretion and therapeutic potential. Basic Clin Pharmacol Toxicol 95:252-262.

Gromada J, Dissing S, and Rorsman P (1996) Desensitization of glucagon-like peptide 1 receptors in insulin-secreting beta TC3 cells: role of PKA-independent mechanisms. Br J Pharmacol 118:769-775.

Gromada J, Holst JJ, and Rorsman P (1998) Cellular regulation of islet hormone secretion by the incretin hormone glucagon-like peptide 1. Pflugers Arch 435 $583-594$.

Gromada J and Rorsman P (2004) New insights into the regulation of glucagon secretion by glucagon-like peptide-1. Horm Metab Res 36:822-829.

Grynkiewicz G, Poenie M, and Tsien RY (1985) A new generation of Ca2+ indicators with greatly improved fluorescence properties. $J$ Biol Chem 260:3440-3450.

Gupta NA, Mells J, Dunham RM, Grakoui A, Handy J, Saxena NK, and Anania FA (2010) Glucagon-like peptide-1 receptor is present on human hepatocytes and has a direct role in decreasing hepatic steatosis in vitro by modulating elements of the insulin signaling pathway. Hepatology 51:1584-1592.

Gustavson S, Burstein A, Valcarce C, Grimes I, and Mjalli A (2014) TTP273, an orally-available glucagon-like peptide-1 (GLP-1) agonist, notably reduces glycemia in subjects with type 2 diabetes mellitus (T2DM). Diabetes 63 (Suppl 1):A41.

Gutniak MK, Juntti-Berggren L, Hellström PM, Guenifi A, Holst JJ, and Efendic $S$ (1996) Glucagon-like peptide I enhances the insulinotropic effect of glibenclamide in NIDDM patients and in the perfused rat pancreas. Diabetes Care $\mathbf{1 9}$ $857-863$

Gutniak M, Orskov C, Holst JJ, Ahrén B, and Efendic S (1992) Antidiabetogenic effect of glucagon-like peptide-1 (7-36)amide in normal subjects and patients with diabetes mellitus. $N$ Engl J Med 326:1316-1322.

Gutzwiller JP, Tschopp S, Bock A, Zehnder CE, Huber AR, Kreyenbuehl M, Gutmann H, Drewe J, Henzen C, Goeke B, et al. (2004) Glucagon-like peptide 1 induce natriuresis in healthy subjects and in insulin-resistant obese men. J Clin Endocrinol Metab 89:3055-3061.

Hadjiyanni I, Siminovitch KA, Danska JS, and Drucker DJ (2010) Glucagon-like peptide-1 receptor signalling selectively regulates murine lymphocyte proliferation and maintenance of peripheral regulatory T cells. Diabetologia 53:730-740.

Halban PA (2004) Cellular sources of new pancreatic beta cells and therapeutic implications for regenerative medicine. Nat Cell Biol 6:1021-1025.

Halic M and Beckmann R (2005) The signal recognition particle and its interactions during protein targeting. Curr Opin Struct Biol 15:116-125.

Hansen HH, Fabricius K, Barkholt P, Niehoff ML, Morley JE, Jelsing J, Pyke C, Knudsen LB, Farr SA, and Vrang N (2015) The GLP-1 receptor agonist liraglutide improves memory function and increases hippocampal CA1 neuronal numbers in a senescence-accelerated mouse model of Alzheimer's disease. J Alzheimers Dis 46 $877-888$.

Haque TS, Lee VG, Riexinger D, Lei M, Malmstrom S, Xin L, Han S, Mapelli C, Cooper CB, Zhang G, et al. (2010) Identification of potent 11mer glucagon-like peptide-1 receptor agonist peptides with novel C-terminal amino acids: homohomophenylalanine analogs. Peptides 31:950-955

Harbeck MC, Chepurny O, Nikolaev VO, Lohse MJ, Holz GG, and Roe MW (2006) Simultaneous optical measurements of cytosolic Ca2+ and cAMP in single cells. Sci STKE 2006:pl6.

Hareter A, Hoffmann E, Bode HP, Göke B, and Göke R (1997) The positive charge of the imidazole side chain of histidine7 is crucial for GLP-1 action. Endocr $J \mathbf{4 4}$ 701-705

Harikumar KG, Ball AM, Sexton PM, and Miller LJ (2010) Importance of lipidexposed residues in transmembrane segment four for family B calcitonin receptor homo-dimerization. Regul Pept 164:113-119.

Harikumar KG, Morfis MM, Lisenbee CS, Sexton PM, and Miller LJ (2006) Constitutive formation of oligomeric complexes between family B G protein-coupled vasoactive intestinal polypeptide and secretin receptors. Mol Pharmacol 69: 363-373

Harikumar KG, Wootten D, Pinon DI, Koole C, Ball AM, Furness SG, Graham B Dong M, Christopoulos A, Miller LJ, et al. (2012) Glucagon-like peptide-1 receptor dimerization differentially regulates agonist signaling but does not affect small molecule allostery. Proc Natl Acad Sci USA 109:18607-18612.

Harkavyi A, Abuirmeileh A, Lever R, Kingsbury AE, Biggs CS, and Whitton PS (2008) Glucagon-like peptide 1 receptor stimulation reverses key deficits in distinct rodent models of Parkinson's disease. J Neuroinflammation 5:19.

Harris PE, Ferrara C, Barba P, Polito T, Freeby M, and Maffei A (2008) VMAT2 gene expression and function as it applies to imaging $\beta$-cell mass. $J$ Mol Med 86:5-16.
Hayes MR, Leichner TM, Zhao S, Lee GS, Chowansky A, Zimmer D, De Jonghe BC, Kanoski SE, Grill HJ, and Bence KK (2011) Intracellular signals mediating the food intake-suppressive effects of hindbrain glucagon-like peptide-1 receptor activation. Cell Metab 13:320-330.

He M, Guan N, Gao WW, Liu Q, Wu XY, Ma DW, Zhong DF, Ge GB, Li C, Chen XY, et al. (2012) A continued saga of Boc5, the first non-peptidic glucagon-like peptide-1 receptor agonist with in vivo activities. Acta Pharmacol Sin 33:148-154

He M, Su H, Gao W, Johansson SM, Liu Q, Wu X, Liao J, Young AA, Bartfai T, and Wang MW (2010) Reversal of obesity and insulin resistance by a non-peptidic glucagon-like peptide-1 receptor agonist in diet-induced obese mice. PLoS One 5: e14205.

Heijboer AC, Frans A, Lomecky M, and Blankenstein MA (2011) Analysis of glucagon-like peptide 1; what to measure? Clin Chim Acta 412:1191-1194.

Heinrich G, Gros P, Lund PK, Bentley RC, and Habener JF (1984) Pre-proglucagon messenger ribonucleic acid: nucleotide and encoded amino acid sequences of the rat pancreatic complementary deoxyribonucleic acid. Endocrinology 115:2176-2181.

Heller RS, Kieffer TJ, and Habener JF (1996) Point mutations in the first and third intracellular loops of the glucagon-like peptide-1 receptor alter intracellular signaling. Biochem Biophys Res Commun 223:624-632.

Heller RS, Kieffer TJ, and Habener JF (1997) Insulinotropic glucagon-like peptide I receptor expression in glucagon-producing alpha-cells of the rat endocrine pancreas. Diabetes 46:785-791.

Hendrick GK, Gjinovci A, Baxter LA, Mojsov S, Wollheim CB, Habener JF, and Weir GC (1993) Glucagon-like peptide-I-(7-37) suppresses hyperglycemia in rats. $\mathrm{Me}$ tabolism 42:1-6.

Heppner KM and Perez-Tilve D (2015) GLP-1 based therapeutics: simultaneously combating T2DM and obesity. Front Neurosci 9:92

Higy M, Junne T, and Spiess M (2004) Topogenesis of membrane proteins at the endoplasmic reticulum. Biochemistry 43:12716-12722.

Hinke SA, Manhart S, Pamir N, Demuth $\mathrm{H}$, W Gelling R, Pederson RA, and McIntosh CHS (2001) Identification of a bioactive domain in the amino-terminus of glucosedependent insulinotropic polypeptide (GIP). Biochim Biophys Acta 1547:143-155.

Hjorth SA, Adelhorst K, Pedersen BB, Kirk O, and Schwartz TW (1994) Glucagon and glucagon-like peptide 1: selective receptor recognition via distinct peptide epitopes. J Biol Chem 269:30121-30124.

Hoang HN, Song K, Hill TA, Derksen DR, Edmonds DJ, Kok WM, Limberakis C, Liras S, Loria PM, Mascitti V, et al. (2015) Short hydrophobic peptides with cyclic constraints are potent glucagon-like peptide-1 receptor (GLP-1R) agonists. J Med Chem 58:4080-4085.

Hoare SR (2005) Mechanisms of peptide and nonpeptide ligand binding to class B G-protein-coupled receptors. Drug Discov Today 10:417-427.

Hogan AE, Tobin AM, Ahern T, Corrigan MA, Gaoatswe G, Jackson R, O'Reilly V, Lynch L, Doherty DG, Moynagh PN, et al. (2011) Glucagon-like peptide-1 (GLP-1) and the regulation of human invariant natural killer T cells: lessons from obesity, diabetes and psoriasis. Diabetologia 54:2745-2754.

Hollenstein K, de Graaf C, Bortolato A, Wang MW, Marshall FH, and Stevens RC (2014) Insights into the structure of class B GPCRs. Trends Pharmacol Sci $\mathbf{3 5}$ $12-22$

Hollenstein K, Kean J, Bortolato A, Cheng RK, Doré AS, Jazayeri A, Cooke RM, Weir M, and Marshall FH (2013) Structure of class B GPCR corticotropin-releasing factor receptor 1. Nature 499:438-443.

Holst JJ (1997) Enteroglucagon. Annu Rev Physiol 59:257-271.

Holst JJ (2007) The physiology of glucagon-like peptide 1. Physiol Rev 87:1409-1439. Holst JJ, Bersani M, Johnsen AH, Kofod H, Hartmann B, and Orskov C (1994) Proglucagon processing in porcine and human pancreas. $J$ Biol Chem 269: 18827-18833.

Holst JJ and Orskov C (2001) Incretin hormones: an update. Scand J Clin Lab Invest Suppl 234:75-85.

Holst JJ, Orskov C, Nielsen OV, and Schwartz TW (1987) Truncated glucagon-like peptide I, an insulin-releasing hormone from the distal gut. FEBS Lett 211 $169-174$

Holz GG (2004) Epac: A new cAMP-binding protein in support of glucagon-like peptide-1 receptor-mediated signal transduction in the pancreatic beta-cell. $\mathrm{Di}$ abetes 53:5-13.

Huang G, Li J, Fu H, Yan Z, Bu G, He X, and Wang Y (2012) Characterization of glucagon-like peptide 1 receptor (GLP1R) gene in chickens: functional analysis, tissue distribution, and identification of its transcript variants. Domest Anim Endocrinol 43:1-15.

Huang CL, Sun L, Moonga BS, and Zaidi M (2006) Molecular physiology and pharmacology of calcitonin. Cell Mol Biol 52:33-43.

Huang Y, Wilkinson GF, and Willars GB (2010) Role of the signal peptide in the synthesis and processing of the glucagon-like peptide-1 receptor. Br J Pharmacol 159:237-251.

Hui H, Nourparvar A, Zhao X, and Perfetti R (2003) Glucagon-like peptide-1 inhibits apoptosis of insulin-secreting cells via a cyclic $5^{\prime}$-adenosine monophosphatedependent protein kinase A- and a phosphatidylinositol 3-kinase-dependent pathway. Endocrinology 144:1444-1455.

Hui $\mathrm{H}$ and Perfetti R (2002) Pancreas duodenum homeobox-1 regulates pancreas development during embryogenesis and islet cell function in adulthood. Eur $J$ Endocrinol 146:129-141.

Ichise M and Harris PE (2010) Imaging of $\beta$-cell mass and function. J Nucl Med 51 1001-1004

Illergård K, Kauko A, and Elofsson A (2011) Why are polar residues within the membrane core evolutionary conserved? Proteins 79:79-91.

Inooka H, Ohtaki T, Kitahara O, Ikegami T, Endo S, Kitada C, Ogi K, Onda H, Fujino M, and Shirakawa M (2001) Conformation of a peptide ligand bound to its G-protein coupled receptor. Nat Struct Biol 8:161-165.

Irwin N, Flatt PR, Patterson S, and Green BD (2010) Insulin-releasing and metabolic effects of small molecule GLP-1 receptor agonist 6,7-dichloro-2-methylsulfonyl-3-Ntert-butylaminoquinoxaline. Eur J Pharmacol 628:268-273. 
Isberg V, de Graaf C, Bortolato A, Cherezov V, Katritch V, Marshall FH, Mordalski S, Pin JP, Stevens RC, Vriend G, et al. (2015) Generic GPCR residue numbers: aligning topology maps while minding the gaps. Trends Pharmacol Sci 36:22-31.

Ishii M, Shibata R, Kondo K, Kambara T, Shimizu Y, Tanigawa T, Bando YK, Nishimura M, Ouchi N, and Murohara T (2014) Vildagliptin stimulates endothelial cell network formation and ischemia-induced revascularization via an endothelial nitric-oxide synthase-dependent mechanism. $J$ Biol Chem 289 $27235-27245$.

Jazayeri A, Doré AS, Lamb D, Krishnamurthy H, Southall SM, Baig AH, Bortolato A, Koglin M, Robertson NJ, Errey JC, et al. (2016) Extra-helical binding site of a glucagon receptor antagonist. Nature 533:274-277.

Jendle J, Rosenstock J, Blonde L, Woo V, Gross J, Jiang H, and Milicevic Z (2014) Better glycemic control and less weight gain with once-weekly dulaglutide vs. oncedaily insulin glargine, both combined with premeal insulin lispro, in type 2 diabetes patients (AWARD-4). Diabetes 63 (Suppl 1):A246.

Jhala US, Canettieri G, Screaton RA, Kulkarni RN, Krajewski S, Reed J, Walker J, Lin X, White M, and Montminy M (2003) cAMP promotes pancreatic beta-cell survival via CREB-mediated induction of IRS2. Genes Dev 17:1575-1580.

Ji Z, Hadac EM, Henne RM, Patel SA, Lybrand TP, and Miller LJ (1997) Direct identification of a distinct site of interaction between the carboxyl-terminal residue of cholecystokinin and the type A cholecystokinin receptor using photoaffinity labeling. J Biol Chem 272:24393-24401.

Jin SL, Han VK, Simmons JG, Towle AC, Lauder JM, and Lund PK (1988) Distribution of glucagonlike peptide I (GLP-I), glucagon, and glicentin in the rat brain: an immunocytochemical study. J Comp Neurol 271:519-532.

Jorgensen R, Kubale V, Vrecl M, Schwartz TW, and Elling CE (2007) Oxyntomodulin differentially affects glucagon-like peptide-1 receptor beta-arrestin recruitment and signaling through Galpha(s). J Pharmacol Exp Ther 322:148-154.

Jorgensen R, Martini L, Schwartz TW, and Elling CE (2005) Characterization of glucagon-like peptide-1 receptor beta-arrestin 2 interaction: a high-affinity receptor phenotype. Mol Endocrinol 19:812-823.

Jorgensen R, Norklit Roed S, Heding A, and Elling CE (2011) Beta-arrestin2 as a competitor for GRK2 interaction with the GLP-1 receptor upon receptor activation. Pharmacology 88:174-181.

Kang G, Joseph JW, Chepurny OG, Monaco M, Wheeler MB, Bos JL, Schwede F, Genieser HG, and Holz GG (2003) Epac-selective cAMP analog 8-pCPT-2'-O-MecAMP as a stimulus for $\mathrm{Ca}^{2+}$-induced $\mathrm{Ca}^{2+}$ release and exocytosis in pancreatic beta-cells. J Biol Chem 278:8279-8285.

Kang G, Leech CA, Chepurny OG, Coetzee WA, and Holz GG (2008) Role of the cAMP sensor Epac as a determinant of KATP channel ATP sensitivity in human pancreatic beta-cells and rat INS-1 cells. J Physiol 586:1307-1319.

Kanse SM, Kreymann B, Ghatei MA, and Bloom SR (1988) Identification and characterization of glucagon-like peptide-1 7-36 amide-binding sites in the rat brain and lung. FEBS Lett 241:209-212.

Kenakin T and Christopoulos A (2013) Signalling bias in new drug discovery: detection, quantification and therapeutic impact. Nat Rev Drug Discov 12:205-216.

Kenakin T and Miller LJ (2010) Seven transmembrane receptors as shapeshifting proteins: the impact of allosteric modulation and functional selectivity on new drug discovery. Pharmacol Rev 62:265-304.

Kenakin T, Watson C, Muniz-Medina V, Christopoulos A, and Novick S (2012) A simple method for quantifying functional selectivity and agonist bias. ACS Chem Neurosci 3:193-203.

Keov P, Sexton PM, and Christopoulos A (2011) Allosteric modulation of G proteincoupled receptors: a pharmacological perspective. Neuropharmacology 60:24-35.

Kim JG, Baggio LL, Bridon DP, Castaigne JP, Robitaille MF, Jetté L, Benquet C, and Drucker DJ (2003) Development and characterization of a glucagon-like peptide 1-albumin conjugate: the ability to activate the glucagon-like peptide 1 receptor in vivo. Diabetes 52:751-759.

Kim D, MacConell L, Zhuang D, Kothare PA, Trautmann M, Fineman M, and Taylor $\mathrm{K}$ (2007) Effects of once-weekly dosing of a long-acting release formulation of exenatide on glucose control and body weight in subjects with type 2 diabetes. Diabetes Care 30:1487-1493

Kim S, Moon M, and Park S (2009) Exendin-4 protects dopaminergic neurons by inhibition of microglial activation and matrix metalloproteinase-3 expression in an animal model of Parkinson's disease. J Endocrinol 202:431-439.

Kitamura T, Nakae J, Kitamura Y, Kido Y, Biggs WH 3rd, Wright CV, White MF, Arden KC, and Accili D (2002) The forkhead transcription factor Foxo1 links insulin signaling to $\mathrm{Pdx} 1$ regulation of pancreatic beta cell growth. J Clin Invest 110 1839-1847.

Kjems LL, Holst JJ, Vølund A, and Madsbad S (2003) The influence of GLP-1 on glucose-stimulated insulin secretion: effects on beta-cell sensitivity in type 2 and nondiabetic subjects. Diabetes 52:380-386.

Knudsen LB (2004) Glucagon-like peptide-1: the basis of a new class of treatment for type 2 diabetes. $J$ Med Chem 47:4128-4134.

Knudsen LB, Kiel D, Teng M, Behrens C, Bhumralkar D, Kodra JT, Holst JJ, Jeppesen CB, Johnson MD, de Jong JC, et al. (2007) Small-molecule agonists for the glucagon-like peptide 1 receptor. Proc Natl Acad Sci USA 104:937-942.

Knudsen LB, Nielsen PF, Huusfeldt PO, Johansen NL, Madsen K, Pedersen FZ, Thøgersen H, Wilken M, and Agersø H (2000) Potent derivatives of glucagon-like peptide-1 with pharmacokinetic properties suitable for once daily administration. $J$ Med Chem 43:1664-1669.

Köchl R, Alken M, Rutz C, Krause G, Oksche A, Rosenthal W, and Schülein R (2002) The signal peptide of the $\mathrm{G}$ protein-coupled human endothelin $\mathrm{B}$ receptor is necessary for translocation of the N-terminal tail across the endoplasmic reticulum membrane. J Biol Chem 277:16131-16138.

Kodera R, Shikata K, Kataoka HU, Takatsuka T, Miyamoto S, Sasaki M, Kajitani N, Nishishita S, Sarai K, Hirota D, et al. (2011) Glucagon-like peptide-1 receptor agonist ameliorates renal injury through its anti-inflammatory action without lowering blood glucose level in a rat model of type 1 diabetes. Diabetologia 54: 965-978.
Koehler JA, Baggio LL, Lamont BJ, Ali S, and Drucker DJ (2009) Glucagon-like peptide-1 receptor activation modulates pancreatitis-associated gene expression but does not modify the susceptibility to experimental pancreatitis in mice. $\mathrm{Di}$ abetes 58:2148-2161.

Koehler JA, Kain T, and Drucker DJ (2011) Glucagon-like peptide-1 receptor activation inhibits growth and augments apoptosis in murine CT26 colon cancer cells. Endocrinology 152:3362-3372.

Kolterman OG, Buse JB, Fineman MS, Gaines E, Heintz S, Bicsak TA, Taylor K, Kim D, Aisporna M, Wang Y, et al. (2003) Synthetic exendin-4 (exenatide) significantly reduces postprandial and fasting plasma glucose in subjects with type 2 diabetes. $J$ Clin Endocrinol Metab 88:3082-3089.

Koole C, Savage EE, Christopoulos A, Miller LJ, Sexton PM, and Wootten D (2013b) Minireview: signal bias, allosterism, and polymorphic variation at the GLP-1R: implications for drug discovery. Mol Endocrinol 27:1234-1244.

Koole C, Pabreja K, Savage EE, Wootten D, Furness SG, Miller LJ, Christopoulos A, and Sexton PM (2013a) Recent advances in understanding GLP-1R (glucagon-like peptide-1 receptor) function. Biochem Soc Trans 41:172-179.

Koole C, Wootten D, Simms J, Miller LJ, Christopoulos A, and Sexton PM (2012a) Second extracellular loop of human glucagon-like peptide-1 receptor (GLP-1R) has a critical role in GLP-1 peptide binding and receptor activation. J Biol Chem 287: $3642-3658$

Koole C, Wootten D, Simms J, Miller LJ, Christopoulos A, and Sexton PM (2015) Differential impact of amino acid substitutions on critical residues of the human glucagon-like peptide-1 receptor involved in peptide activity and small-molecule allostery. J Pharmacol Exp Ther 353:52-63.

Koole C, Wootten D, Simms J, Savage EE, Miller LJ, Christopoulos A, and Sexton PM (2012b) Second extracellular loop of human glucagon-like peptide-1 receptor (GLP-1R) differentially regulates orthosteric but not allosteric agonist binding and function. J Biol Chem 287:3659-3673.

Koole C, Wootten D, Simms J, Valant C, Miller LJ, Christopoulos A, and Sexton PM (2011) Polymorphism and ligand dependent changes in human glucagon-like peptide-1 receptor (GLP-1R) function: allosteric rescue of loss of function mutation. Mol Pharmacol 80:486-497.

Koole C, Wootten D, Simms J, Valant C, Sridhar R, Woodman OL, Miller LJ, Summers RJ, Christopoulos A, and Sexton PM (2010) Allosteric ligands of the glucagonlike peptide 1 receptor (GLP-1R) differentially modulate endogenous and exogenous peptide responses in a pathway-selective manner: implications for drug screening. Mol Pharmacol 78:456-465.

Koth CM, Murray JM, Mukund S, Madjidi A, Minn A, Clarke HJ, Wong T, Chiang V, Luis E, Estevez A, et al. (2012) Molecular basis for negative regulation of the glucagon receptor. Proc Natl Acad Sci USA 109:14393-14398.

Kreymann B, Williams G, Ghatei MA, and Bloom SR (1987) Glucagon-like peptide-1 7-36: a physiological incretin in man. Lancet 2:1300-1304.

Kuna RS, Girada SB, Asalla S, Vallentyne J, Maddika S, Patterson JT, Smiley DL, DiMarchi RD, and Mitra P (2013) Glucagon-like peptide-1 receptor-mediated endosomal cAMP generation promotes glucose-stimulated insulin secretion in pancreatic $\beta$-cells. Am J Physiol Endocrinol Metab 305:E161-E170.

Kung MP, Hou C, Lieberman BP, Oya S, Ponde DE, Blankemeyer E, Skovronsky D, Kilbourn MR, and Kung HF (2008) In vivo imaging of beta-cell mass in rats using 18F-FP-(+)-DTBZ: a potential PET ligand for studying diabetes mellitus. J Nucl Med 49:1171-1176.

Kwan EP, Xie L, Sheu L, Ohtsuka T, and Gaisano HY (2007) Interaction between Munc13-1 and RIM is critical for glucagon-like peptide-1 mediated rescue of exocytotic defects in Munc13-1 deficient pancreatic beta-cells. Diabetes 56 2579-2588.

La Barre J (1932) Sur les possibilite's d'un traitement du diabète par l'incrétine. Bull Acad R Med Belg 12:14.

Landa LR Jr, Harbeck M, Kaihara K, Chepurny O, Kitiphongspattana K, Graf O, Nikolaev VO, Lohse MJ, Holz GG, and Roe MW (2005) Interplay of $\mathrm{Ca}^{2+}$ and cAMP signaling in the insulin-secreting MIN6 beta-cell line. $J$ Biol Chem 280: 31294-31302.

Lau J, Bloch P, Schäffer L, Pettersson I, Spetzler J, Kofoed J, Madsen K, Knudsen LB, McGuire J, Steensgaard DB, et al. (2015) Discovery of the once-weekly glucagon-like peptide-1 (GLP-1) analogue semaglutide. J Med Chem 58:7370-7380.

Lawrence MC, Bhatt HS, and Easom RA (2002) NFAT regulates insulin gene promoter activity in response to synergistic pathways induced by glucose and glucagon-like peptide-1. Diabetes 51:691-698.

Le WD, Chen S, and Jankovic J (2009) Etiopathogenesis of Parkinson disease: a new beginning? Neuroscientist 15:28-35.

Leach K, Sexton PM, and Christopoulos A (2007) Allosteric GPCR modulators: taking advantage of permissive receptor pharmacology. Trends Pharmacol Sci 28: 382-389.

Lean ME, Carraro R, Finer N, Hartvig H, Lindegaard ML, Rössner S, Van Gaal L and Astrup A; NN8022-1807 Investigators (2014) Tolerability of nausea and vomiting and associations with weight loss in a randomized trial of liraglutide in obese, non-diabetic adults. Int J Obes 38:689-697.

$\mathrm{Li} \mathrm{N}$, Lu J, and Willars GB (2012a) Allosteric modulation of the activity of the glucagon-like peptide-1 (GLP-1) metabolite GLP-1 9-36 amide at the GLP-1 receptor. PLoS One 7:e47936.

Li Y, Cao X, Li LX, Brubaker PL, Edlund H, and Drucker DJ (2005) beta-Cell Pdx1 expression is essential for the glucoregulatory, proliferative, and cytoprotective actions of glucagon-like peptide-1. Diabetes 54:482-491.

Li Y, Chigurupati S, Holloway HW, Mughal M, Tweedie D, Bruestle DA, Mattson MP, Wang Y, Harvey BK, Ray B, et al. (2012b) Exendin-4 ameliorates motor neuron degeneration in cellular and animal models of amyotrophic lateral sclerosis. PLoS One 7:e32008.

Li Y, Perry T, Kindy MS, Harvey BK, Tweedie D, Holloway HW, Powers K, Shen H, Egan JM, Sambamurti K, et al. (2009) GLP-1 receptor stimulation preserves primary cortical and dopaminergic neurons in cellular and rodent models of stroke and parkinsonism. Proc Natl Acad Sci USA 106:1285-1290. 
Li Y, Tweedie D, Mattson MP, Holloway HW, and Greig NH (2010) Enhancing the GLP-1 receptor signaling pathway leads to proliferation and neuroprotection in human neuroblastoma cells. J Neurochem 113:1621-1631.

Light PE, Manning Fox JE, Riedel MJ, and Wheeler MB (2002) Glucagon-like peptide-1 inhibits pancreatic ATP-sensitive potassium channels via a protein kinase A- and ADP-dependent mechanism. Mol Endocrinol 16:2135-2144.

Liu Q, Li N, Yuan Y, Lu H, Wu X, Zhou C, He M, Su H, Zhang M, Wang J, et al. (2012) Cyclobutane derivatives as novel nonpeptidic small molecule agonists of glucagonlike peptide-1 receptor. J Med Chem 55:250-267.

Locatelli-Hoops S, Yeliseev AA, Gawrisch K, and Gorshkova I (2013) Surface plasmon resonance applied to $\mathrm{G}$ protein-coupled receptors. Biomed Spectrosc Imaging 2 155-181

López de Maturana R and Donnelly D (2002) The glucagon-like peptide-1 receptor binding site for the N-terminus of GLP-1 requires polarity at Asp198 rather than negative charge. FEBS Lett 530:244-248.

López de Maturana R, Treece-Birch J, Abidi F, Findlay JB, and Donnelly D (2004) Met-204 and Tyr-205 are together important for binding GLP-1 receptor agonists but not their N-terminally truncated analogues. Protein Pept Lett 11:15-22.

López de Maturana R, Willshaw A, Kuntzsch A, Rudolph R, and Donnelly D (2003) The isolated N-terminal domain of the glucagon-like peptide-1 (GLP-1) receptor binds exendin peptides with much higher affinity than GLP-1. J Biol Chem 278 10195-10200.

Lord K, Radcliffe J, Gallagher PR, Adzick NS, Stanley CA, and De León DD (2015) High risk of diabetes and neurobehavioral deficits in individuals with surgically treated hyperinsulinism. J Clin Endocrinol Metab 100:4133-4139.

Lorenz M, Evers A, and Wagner M (2013) Recent progress and future options in the development of GLP-1 receptor agonists for the treatment of diabesity. Bioorg Med Chem Lett 23:4011-4018.

Lund A, Knop FK, and Vilsbøll T (2011) Emerging GLP-1 receptor agonists. Expert Opin Emerg Drugs 16:607-618.

Lund PK (2005) The discovery of glucagon-like peptide 1. Regul Pept 128:93-96.

Lund PK, Goodman RH, Dee PC, and Habener JF (1982) Pancreatic preproglucagon cDNA contains two glucagon-related coding sequences arranged in tandem. Proc Natl Acad Sci USA 79:345-349.

Lund PK, Goodman RH, and Habener JF (1981) Pancreatic pre-proglucagons are encoded by two separate mRNAs. J Biol Chem 256:6515-6518.

Lv C, Liu X, Liu H, Chen T, and Zhang W (2014) Geniposide attenuates mitochondrial dysfunction and memory deficits in APP/PS1 transgenic mice. Curr Alzheimer Res 11:580-587.

MacDonald PE, Joseph JW, and Rorsman P (2005) Glucose-sensing mechanisms in pancreatic beta-cells. Philos Trans R Soc Lond B Biol Sci 360:2211-2225.

MacDonald PE, Wang X, Xia F, El-kholy W, Targonsky ED, Tsushima RG, and Wheeler MB (2003) Antagonism of rat beta-cell voltage-dependent $\mathrm{K}^{+}$currents by exendin 4 requires dual activation of the cAMP/protein kinase A and phosphatidylinositol 3-kinase signaling pathways. J Biol Chem 278:52446-52453.

MacLusky NJ, Cook S, Scrocchi L, Shin J, Kim J, Vaccarino F, Asa SL, and Drucker DJ (2000) Neuroendocrine function and response to stress in mice with complete disruption of glucagon-like peptide-1 receptor signaling. Endocrinology 141:752-762.

Madsbad S (2016) Review of head-to-head comparisons of glucagon-like peptide-1 receptor agonists. Diabetes Obes Metab 18:317-332.

Madsbad S, Kielgast U, Asmar M, Deacon CF, Torekov SS, and Holst JJ (2011) An overview of once-weekly glucagon-like peptide-1 receptor agonists: available efficacy and safety data and perspectives for the future. Diabetes Obes Metab 13 394-407.

Madsen LW, Knauf JA, Gotfredsen C, Pilling A, Sjögren I, Andersen S, Andersen L, de Boer AS, Manova K, Barlas A, et al. (2012) GLP-1 receptor agonists and the thyroid: C-cell effects in mice are mediated via the GLP-1 receptor and not associated with RET activation. Endocrinology 153:1538-1547.

Madsen K, Knudsen LB, Agersoe H, Nielsen PF, Thøgersen H, Wilken M, and Johansen NL (2007) Structure-activity and protraction relationship of longacting glucagon-like peptide-1 derivatives: importance of fatty acid length, polarity, and bulkiness. $J$ Med Chem 50:6126-6132.

Malaisse WJ, Louchami K, and Sener A (2009) Noninvasive imaging of pancreatic beta cells. Nat Rev Endocrinol 5:394-400.

Maljaars PW, Peters HP, Mela DJ, and Masclee AA (2008) Ileal brake: a sensible food target for appetite control: a review. Physiol Behav 95:271-281.

Malone J, Trautmann M, Wilhelm K, Taylor K, and Kendall DM (2009) Exenatide once weekly for the treatment of type 2 diabetes. Expert Opin Investig Drugs 18 359-367.

Manandhar B and Ahn JM (2015) Glucagon-like peptide-1 (GLP-1) analogs: recent advances, new possibilities, and therapeutic implications. J Med Chem 58 1020-1037.

Manandhar B, Lo S-T, Murage E, Lin M, Sun X, and Ahn J-M (2013) Targeting GLP1R for non-invasive assessment of pancreatic beta-cell mass, in Peptides Across The Pacific: Proceedings of the 23rd American Peptide Symposium (Lebl M ed); 2013 June 22-27; Big Island, Hawaii. pp 172-173, American Peptide Society.

Mangmool S, Hemplueksa P, Parichatikanond W, and Chattipakorn N (2015) Epac is required for GLP-1R-mediated inhibition of oxidative stress and apoptosis in cardiomyocytes. Mol Endocrinol 29:583-596.

Mann R, Nasr N, Hadden D, Sinfield J, Abidi F, Al-Sabah S, de Maturana RL, Treece-Birch J, Willshaw A, and Donnelly D (2007) Peptide binding at the GLP-1 receptor. Biochem Soc Trans 35:713-716.

Mann RJ, Al-Sabah S, de Maturana RL, Sinfield JK, and Donnelly D (2010a) Functional coupling of Cys-226 and Cys-296 in the glucagon-like peptide-1 (GLP-1) receptor indicates a disulfide bond that is close to the activation pocket. Peptides 31:2289-2293.

Mann RJ, Nasr NE, Sinfield JK, Paci E, and Donnelly D (2010b) The major determinant of exendin-4/glucagon-like peptide 1 differential affinity at the rat glucagon-like peptide 1 receptor $\mathrm{N}$-terminal domain is a hydrogen bond from SER32 of exendin-4. Br J Pharmacol 160:1973-1984.
Mapelli C, Natarajan SI, Meyer JP, Bastos MM, Bernatowicz MS, Lee VG, Pluscec J, Riexinger DJ, Sieber-McMaster ES, Constantine KL, et al (2009) Eleven amino acid glucagon-like peptide-1 receptor agonists with antidiabetic activity. $J \mathrm{Med}$ Chem 52:7788-7799.

Marsk R, Jonas E, Rasmussen F, and Näslund E (2010) Nationwide cohort study of post-gastric bypass hypoglycaemia including 5,040 patients undergoing surgery for obesity in 1986-2006 in Sweden. Diabetologia 53:2307-2311.

Mathi SK, Chan Y, Li X, and Wheeler MB (1997) Scanning of the glucagon-like peptide-1 receptor localizes $\mathrm{G}$ protein-activating determinants primarily to the $\mathrm{N}$ terminus of the third intracellular loop. Mol Endocrinol 11:424-432.

Mattson MP, Perry T, and Greig NH (2003) Learning from the gut. Nat Med $\mathbf{9}$ $1113-1115$

Matveyenko AV and Butler PC (2008) Relationship between $\beta$-cell mass and diabetes onset. Diabetes Obes Metab 10 (Suppl 4):23-31.

Maurel D, Comps-Agrar L, Brock C, Rives ML, Bourrier E, Ayoub MA, Bazin H, Tinel N, Durroux T, Prézeau L, et al. (2008) Cell-surface protein-protein interaction analysis with time-resolved FRET and snap-tag technologies: application to GPCR oligomerization. Nat Methods 5:561-567.

May LT, Leach K, Sexton PM, and Christopoulos A (2007) Allosteric modulation of G protein-coupled receptors. Annu Rev Pharmacol Toxicol 47:1-51.

McClean PL and Hölscher C (2014) Lixisenatide, a drug developed to treat type 2 diabetes, shows neuroprotective effects in a mouse model of Alzheimer's disease. Neuropharmacology 86:241-258.

McClean PL, Parthsarathy V, Faivre E, and Hölscher C (2011) The diabetes drug liraglutide prevents degenerative processes in a mouse model of Alzheimer's disease. $J$ Neurosci 31:6587-6594.

McIntyre N, Holdsworth CD, and Turner DS (1964) New interpretation of oral glucose tolerance. Lancet 2:20-21.

Meier JJ (2012) GLP-1 receptor agonists for individualized treatment of type 2 diabetes mellitus. Nat Rev Endocrinol 8:728-742.

Miller LJ, Chen Q, Lam PC, Pinon DI, Sexton PM, Abagyan R, and Dong M (2011) Refinement of glucagon-like peptide 1 docking to its intact receptor using midregion photolabile probes and molecular modeling. J Biol Chem 286:15895-15907.

Mima A, Hiraoka-Yamomoto J, Li Q, Kitada M, Li C, Geraldes P, Matsumoto M, Mizutani K, Park K, Cahill C, et al. (2012) Protective effects of GLP-1 on glomerular endothelium and its inhibition by $\mathrm{PKC} \beta$ activation in diabetes. Diabetes 61 : 2967-2979.

Miranda LP, Winters KA, Gegg CV, Patel A, Aral J, Long J, Zhang J, Diamond S, Guido M, Stanislaus S, et al. (2008) Design and synthesis of conformationally constrained glucagon-like peptide-1 derivatives with increased plasma stability and prolonged in vivo activity. J Med Chem 51:2758-2765.

Mojsov S (1992) Structural requirements for biological activity of glucagon-like peptide-I. Int $J$ Pept Protein Res 40:333-343.

Mojsov S, Heinrich G, Wilson IB, Ravazzola M, Orci L, and Habener JF (1986) Preproglucagon gene expression in pancreas and intestine diversifies at the level of post-translational processing. J Biol Chem 261:11880-11889.

Mojsov S, Weir GC, and Habener JF (1987) Insulinotropin: glucagon-like peptide I (737) co-encoded in the glucagon gene is a potent stimulator of insulin release in the perfused rat pancreas. J Clin Invest 79:616-619.

Montanya E (2012) A comparison of currently available GLP-1 receptor agonists for the treatment of type 2 diabetes. Expert Opin Pharmacother 13:1451-1467.

Montrose-Rafizadeh C, Avdonin P, Garant MJ, Rodgers BD, Kole S, Yang H, Levine MA, Schwindinger W, and Bernier M (1999) Pancreatic glucagon-like peptide-1 receptor couples to multiple G proteins and activates mitogen-activated protein kinase pathways in Chinese hamster ovary cells. Endocrinology 140 $1132-1140$

Montrose-Rafizadeh C, Yang H, Rodgers BD, Beday A, Pritchette LA, and Eng J (1997) High potency antagonists of the pancreatic glucagon-like peptide-1 receptor. J Biol Chem 272·21201-21206.

Moon MJ, Kim HY, Park S, Kim DK, Cho EB, Park CR, You DJ, Hwang JI, Kim K Choe H, et al. (2012) Evolutionarily conserved residues at glucagon-like peptide-1 GLP-1) receptor core confer ligand-induced receptor activation. J Biol Chem 287: $3873-3884$

Moon MJ, Lee YN, Park S, Reyes-Alcaraz A, Hwang JI, Millar RP, Choe H, and Seong JY (2015) Ligand binding pocket formed by evolutionarily conserved residues in the glucagon-like peptide-1 (GLP-1) receptor core domain. J Biol Chem 290: $5696-5706$.

Moore A (2009) Advances in beta-cell imaging. Eur J Radiol 70:254-257.

Moore B (1906) On the treatment of diabetus mellitus by acid extract of duodenal mucous membrane. Biochem $J$ 1:28-38.

Moretto TJ, Milton DR, Ridge TD, Macconell LA, Okerson T, Wolka AM, and Brodows RG (2008) Efficacy and tolerability of exenatide monotherapy over 24 weeks in antidiabetic drug-naive patients with type 2 diabetes: a randomized double-blind, placebo-controlled, parallel-group study. Clin Ther 30:1448-1460.

Moroo I, Yamada T, Makino H, Tooyama I, McGeer PL, McGeer EG, and Hirayama K (1994) Loss of insulin receptor immunoreactivity from the substantia nigra pars compacta neurons in Parkinson's disease. Acta Neuropathol 87:343-348.

Morris JK, Bomhoff GL, Gorres BK, Davis VA, Kim J, Lee PP, Brooks WM, Gerhard GA, Geiger PC, and Stanford JA (2011) Insulin resistance impairs nigrostriatal dopamine function. Exp Neurol 231:171-180.

Morris LC, Nance KD, Gentry PR, Days EL, Weaver CD, Niswender CM, Thompson AD, Jones CK, Locuson CW, Morrison RD, et al. (2014) Discovery of (S)-2-cyclopentyl$\mathrm{N}$-((1-isopropylpyrrolidin2-yl)-9-methyl-1-oxo-2,9-dihydro-1H-pyrrido[3,4-b]indole-4carboxamide (VU0453379): a novel, CNS penetrant glucagon-like peptide 1 receptor GLP-1R) positive allosteric modulator (PAM). J Med Chem 57:10192-10197.

Morris JK, Zhang H, Gupte AA, Bomhoff GL, Stanford JA, and Geiger PC (2008) Measures of striatal insulin resistance in a 6-hydroxydopamine model of Parkinson's disease. Brain Res 1240:185-195. 
Mudaliar S and Henry RR (2010) Effects of incretin hormones on beta-cell mass and function, body weight, and hepatic and myocardial function. Am J Med 123: S19-S27.

Mukai E, Toyoda K, Kimura H, Kawashima H, Fujimoto H, Ueda M, Temma T, Hirao K, Nagakawa K, Saji H, et al. (2009) GLP-1 receptor antagonist as a potential probe for pancreatic beta-cell imaging. Biochem Biophys Res Commun 389: $523-526$.

Mullard A (2014) Once-yearly device takes on daily and weekly diabetes drugs. Nat Biotechnol 32:1178.

Murage EN, Gao G, Bisello A, and Ahn J-M (2010) Development of potent glucagonlike peptide-1 agonists with high enzyme stability via introduction of multiple lactam bridges. J Med Chem 53:6412-6420.

Murage EN, Schroeder JC, Beinborn M, and Ahn JM (2008) Search for alpha-helical propensity in the receptor-bound conformation of glucagon-like peptide-1. Bioorg Med Chem 16:10106-10112.

Murthy R, Harris P, Simpson N, Van Heertum R, Leibel R, Mann JJ, and Parsey R (2008) Whole body [11C]-dihydrotetrabenazine imaging of baboons: biodistribution and human radiation dosimetry estimates. Eur J Nucl Med Mol Imaging 35:790-797.

Myers GA, Gacek DA, Peterson EM, Fox CB, and Harris JM (2012) Microscopic rates of peptide-phospholipid bilayer interactions from single-molecule residence times. $J$ Am Chem Soc 134:19652-19660.

Nauck MA, Baller B, and Meier JJ (2004) Gastric inhibitory polypeptide and glucagon-like peptide- 1 in the pathogenesis of type 2 diabetes. Diabetes $\mathbf{5 3}$ (Suppl 3):S190-S196.

Nauck MA, Büsing M, Orskov C, Siegel EG, Talartschik J, Baartz A, Baartz T, Hopt UT, Becker HD, and Creutzfeldt W (1993a) Preserved incretin effect in type 1 diabetic patients with end-stage nephropathy treated by combined heterotopic pancreas and kidney transplantation. Acta Diabetol 30:39-45.

Nauck MA and Friedrich N (2013) Do GLP-1-based therapies increase cancer risk? Diabetes Care 36 (Suppl 2):S245-S252.

Nauck MA, Heimesaat MM, Orskov C, Holst JJ, Ebert R, and Creutzfeldt W (1993b) Preserved incretin activity of glucagon-like peptide 1 [7-36 amide] but not of synthetic human gastric inhibitory polypeptide in patients with type-2 diabetes mellitus. J Clin Invest 91:301-307.

Nauck MA, Kemmeries G, Holst JJ, and Meier JJ (2011) Rapid tachyphylaxis of the glucagon-like peptide 1-induced deceleration of gastric emptying in humans. $\mathrm{Di}$ abetes 60:1561-1565.

Nauck MA, Kleine N, Orskov C, Holst JJ, Willms B, and Creutzfeldt W (1993c) Normalization of fasting hyperglycaemia by exogenous glucagon-like peptide 1 (736 amide) in type 2 (non-insulin-dependent) diabetic patients. Diabetologia 36: $741-744$.

Nauck MA, Niedereichholz U, Ettler R, Holst JJ, Orskov C, Ritzel R, and Schmiegel WH (1997) Glucagon-like peptide 1 inhibition of gastric emptying outweighs its insulinotropic effects in healthy humans. Am J Physiol 273:E981-E988.

Nauck MA, Petrie JR, Sesti G, Mannucci E, Courrèges JP, Lindegaard ML, Jensen CB, and Atkin SL; Study 1821 Investigators (2016) A Phase 2, randomized, dosefinding study of the novel once-weekly human GLP-1 analog, semaglutide, compared with placebo and open-label liraglutide in patients with type 2 diabetes. Diabetes Care 39:231-241.

Nauck MPJ and Sesti G (2012) The once-weekly human GLP1 analogue semaglutide provides significant reductions in HbA1c and body weight in patients with type 2 diabetes. Diabetologia 55 (Suppl 1):S7.

Neidigh JW, Fesinmeyer RM, Prickett KS, and Andersen NH (2001) Exendin-4 and glucagon-like-peptide-1: NMR structural comparisons in the solution and micelleassociated states. Biochemistry 40:13188-13200.

Neumann JM, Couvineau A, Murail S, Lacapère JJ, Jamin N, and Laburthe M (2008) Class-B GPCR activation: is ligand helix-capping the key? Trends Biochem Sci 33: 314-319.

Neumiller JJ, Sonnett TE, Wood LD, Setter SM, and Campbell RK (2010) Pharmacology, efficacy and safety of liraglutide in the management of type 2 diabetes Diabetes Metab Syndr Obes 3:215-226.

Nielsen H, Engelbrecht J, Brunak S, and von Heijne G (1997) Identification of prokaryotic and eukaryotic signal peptides and prediction of their cleavage sites. Protein Eng 10:1-6.

Nikolaev VO, Bünemann M, Hein L, Hannawacker A, and Lohse MJ (2004) Novel single chain cAMP sensors for receptor-induced signal propagation. $J$ Biol Chem 279:37215-37218

Nikolaidis LA, Elahi D, Hentosz T, Doverspike A, Huerbin R, Zourelias L, Stolarski C, Shen YT, and Shannon RP (2004a) Recombinant glucagon-like peptide-1 in creases myocardial glucose uptake and improves left ventricular performance in conscious dogs with pacing-induced dilated cardiomyopathy. Circulation 110: 955-961.

Nikolaidis LA, Mankad S, Sokos GG, Miske G, Shah A, Elahi D, and Shannon RP (2004b) Effects of glucagon-like peptide-1 in patients with acute myocardial infarction and left ventricular dysfunction after successful reperfusion. Circulation 109:962-965

Nolte WM, Fortin JP, Stevens BD, Aspnes GE, Griffith DA, Hoth LR, Ruggeri RB, Mathiowetz AM, Limberakis C, Hepworth D, et al. (2014) A potentiator of orthosteric ligand activity at GLP-1R acts via covalent modification. Nat Chem Biol 10:629-631.

Norris SL, Lee N, Thakurta S, and Chan BK (2009) Exenatide efficacy and safety: a systematic review. Diabet Med 26:837-846.

Noyan-Ashraf MH, Momen MA, Ban K, Sadi AM, Zhou YQ, Riazi AM, Baggio LL, Henkelman RM, Husain M, and Drucker DJ (2009) GLP-1R agonist liraglutide activates cytoprotective pathways and improves outcomes after experimental myocardial infarction in mice. Diabetes 58:975-983.

Nyström T, Gutniak MK, Zhang Q, Zhang F, Holst JJ, Ahrén B, and Sjöholm A (2004) Effects of glucagon-like peptide-1 on endothelial function in type 2 diabetes patients with stable coronary artery disease. Am J Physiol Endocrinol Metab 287: E1209-E1215.
Oka J, Suzuki E, and Kondo Y (2000) Endogenous GLP-1 is involved in beta-amyloid protein-induced memory impairment and hippocampal neuronal death in rats. Brain Res 878:194-198.

Orskov C, Bersani M, Johnsen AH, Højrup P, and Holst JJ (1989) Complete sequences of glucagon-like peptide-1 from human and pig small intestine. $J$ Biol Chem 264:12826-12829.

Orskov C, Holst JJ, and Nielsen OV (1988) Effect of truncated glucagon-like peptide1 [proglucagon-(78-107) amide] on endocrine secretion from pig pancreas, antrum, and nonantral stomach. Endocrinology 123:2009-2013.

Orskov C, Holst JJ, Poulsen SS, and Kirkegaard P (1987) Pancreatic and intestinal processing of proglucagon in man. Diabetologia 30:874-881.

Ørskov C, Rabenhøj L, Wettergren A, Kofod H, and Holst JJ (1994) Tissue and plasma concentrations of amidated and glycine-extended glucagon-like peptide I in humans. Diabetes 43:535-539.

Ozaki N, Shibasaki T, Kashima Y, Miki T, Takahashi K, Ueno H, Sunaga Y, Yano H, Matsuura Y, Iwanaga T, et al. (2000) cAMP-GEFII is a direct target of cAMP in regulated exocytosis. Nat Cell Biol 2:805-811.

Pal K, Melcher K, and Xu HE (2012) Structure and mechanism for recognition of peptide hormones by class B G-protein-coupled receptors. Acta Pharmacol Sin 33: 300-311.

Palczewski K, Kumasaka T, Hori T, Behnke CA, Motoshima H, Fox BA, Le Trong I, Teller DC, Okada T, Stenkamp RE, et al. (2000) Crystal structure of rhodopsin: a G protein-coupled receptor. Science 289:739-745.

Panjwani N, Mulvihill EE, Longuet C, Yusta B, Campbell JE, Brown TJ, Streutker C, Holland D, Cao X, Baggio LL, et al. (2013) GLP-1 receptor activation indirectly reduces hepatic lipid accumulation but does not attenuate development of atherosclerosis in diabetic male ApoE(-/-) mice. Endocrinology 154:127-139.

Park CW, Kim HW, Ko SH, Lim JH, Ryu GR, Chung HW, Han SW, Shin S.J, Bang BK, Breyer MD, et al. (2007) Long-term treatment of glucagon-like peptide-1 analog exendin-4 ameliorates diabetic nephropathy through improving metabolic anomalies in db/db mice. J Am Soc Nephrol 18:1227-1238.

Parks M and Rosebraugh C (2010) Weighing risks and benefits of liraglutide: the FDA's review of a new antidiabetic therapy. N Engl J Med 362:774-777.

Parthier C, Kleinschmidt M, Neumann P, Rudolph R, Manhart S, Schlenzig D, Fanghänel J, Rahfeld JU, Demuth HU, and Stubbs MT (2007) Crystal structure of the incretin-bound extracellular domain of a G protein-coupled receptor. Proc Natl Acad Sci USA 104:13942-13947.

Parthier C, Reedtz-Runge S, Rudolph R, and Stubbs MT (2009) Passing the baton in class B GPCRs: peptide hormone activation via helix induction? Trends Biochem Sci 34:303-310.

Patterson JT, Li P, Day JW, Gelfanov VM, and Dimarchi RD (2013) A hydrophobic site on the GLP-1 receptor extracellular domain orients the peptide ligand for signal transduction. Mol Metab 2:86-91.

Patterson JT, Ottaway N, Gelfanov VM, Smiley DL, Perez-Tilve D, Pfluger PT, Tschöp MH, and Dimarchi RD (2011) A novel human-based receptor antagonist of sustained action reveals body weight control by endogenous GLP-1. ACS Chem Biol 6:135-145.

Patti ME, McMahon G, Mun EC, Bitton A, Holst JJ, Goldsmith J, Hanto DW, Callery M, Arky R, Nose V, et al. (2005) Severe hypoglycaemia post-gastric bypass requiring partial pancreatectomy: evidence for inappropriate insulin secretion and pancreatic islet hyperplasia. Diabetologia 48:2236-2240.

Pattou F, Kerr-Conte J, and Wild D (2010) GLP-1-receptor scanning for imaging of human beta cells transplanted in muscle. $N$ Engl $J$ Med 363:1289-1290.

Paul SK, Klein K, Maggs D, and Best JH (2015) The association of the treatment with glucagon-like peptide-1 receptor agonist exenatide or insulin with cardiovascular outcomes in patients with type 2 diabetes: a retrospective observational study. Cardiovasc Diabetol 14:10.

Pawson AJ, Sharman JL, Benson HE, Faccenda E, Alexander SP, Buneman OP, Davenport AP, McGrath JC, Peters JA, Southan C, et al.; NC-IUPHAR (2014) The IUPHAR/BPS Guide to PHARMACOLOGY: an expert-driven knowledgebase of drug targets and their ligands. Nucleic Acids Res 42:D1098-D1106.

Perfetti R, Zhou J, Doyle ME, and Egan JM (2000) Glucagon-like peptide-1 induces cell proliferation and pancreatic-duodenum homeobox-1 expression and increases endocrine cell mass in the pancreas of old, glucose-intolerant rats. Endocrinology 141:4600-4605.

Perley MJ and Kipnis DM (1967) Plasma insulin responses to oral and intravenous glucose: studies in normal and diabetic sujbjects. J Clin Invest 46:1954-1962.

Perret J, Van Craenenbroeck M, Langer I, Vertongen P, Gregoire F, Robberecht P, and Waelbroeck M (2002) Mutational analysis of the glucagon receptor: similarities with the vasoactive intestinal peptide (VIP)/pituitary adenylate cyclase-activating peptide (PACAP)/secretin receptors for recognition of the ligand's third residue. Biochem J 362:389-394.

Perry T, Haughey NJ, Mattson MP, Egan JM, and Greig NH (2002) Protection and reversal of excitotoxic neuronal damage by glucagon-like peptide-1 and exendin-4. J Pharmacol Exp Ther 302:881-888.

Perry T, Lahiri DK, Sambamurti K, Chen D, Mattson MP, Egan JM, and Greig NH (2003) Glucagon-like peptide-1 decreases endogenous amyloid-beta peptide (Abeta) levels and protects hippocampal neurons from death induced by Abeta and iron. $J$ Neurosci Res 72:603-612.

Peyot ML, Gray JP, Lamontagne J, Smith PJ, Holz GG, Madiraju SR, Prentki M, and Heart E (2009) Glucagon-like peptide-1 induced signaling and insulin secretion do not drive fuel and energy metabolism in primary rodent pancreatic betacells. PLoS One 4:e6221.

Plamboeck A, Holst JJ, Carr RD, and Deacon CF (2005) Neutral endopeptidase 24.11 and dipeptidyl peptidase IV are both mediators of the degradation of glucagon-like peptide 1 in the anaesthetised pig. Diabetologia 48:1882-1890.

Pocai A (2013) Action and therapeutic potential of oxyntomodulin. Mol Metab 3 241-251.

Pratley RE, Nauck MA, Barnett AH, Feinglos MN, Ovalle F, Harman-Boehm I, Ye J, Scott R, Johnson S, Stewart M, et al.; HARMONY 7 Study Group (2014) Once- 
weekly albiglutide versus once-daily liraglutide in patients with type 2 diabetes inadequately controlled on oral drugs (HARMONY 7): a randomised, open-label, multicentre, non-inferiority phase 3 study. Lancet Diabetes Endocrinol 2:289-297.

Prentki M and Matschinsky FM (1987) Ca2+, cAMP, and phospholipid-derived messengers in coupling mechanisms of insulin secretion. Physiol Rev 67: $1185-1248$.

Pressley JC, Louis ED, Tang MX, Cote L, Cohen PD, Glied S, and Mayeux R (2003) The impact of comorbid disease and injuries on resource use and expenditures in parkinsonism. Neurology 60:87-93.

Prévost M, Vertongen P, Raussens V, Roberts DJ, Cnudde J, Perret J, and Waelbroeck M (2010) Mutational and cysteine scanning analysis of the glucagon receptor N-terminal domain. J Biol Chem 285:30951-30958.

Pridal L, Ingwersen SH, Larsen FS, Holst JJ, Adelhorst K, and Kirk O (1995) Comparison of sandwich enzyme-linked immunoadsorbent assay and radioimmunoassay for determination of exogenous glucagon-like peptide-1(7-36)amide in plasma. J Pharm Biomed Anal 13:841-850.

Pyke C, Heller RS, Kirk RK, Ørskov C, Reedtz-Runge S, Kaastrup P, Hvelplund A Bardram L, Calatayud D, and Knudsen LB (2014) GLP-1 receptor localization in monkey and human tissue: novel distribution revealed with extensively validated monoclonal antibody. Endocrinology 155:1280-1290.

Qian F, Ni N, Burton LS, Wang YF, Desikan S, Hussain M, and Smith RL (2009) Sustained release subcutaneous delivery of BMS-686117, a GLP-1 receptor peptide agonist, via a zinc adduct. Int $J$ Pharm 374:46-52.

Quoyer J, Longuet C, Broca C, Linck N, Costes S, Varin E, Bockaert J, Bertrand G, and Dalle S (2010) GLP-1 mediates antiapoptotic effect by phosphorylating Bad through a beta-arrestin 1-mediated ERK1/2 activation in pancreatic beta-cells. $J$ Biol Chem 285:1989-2002.

Raab EL, Vuguin PM, Stoffers DA, and Simmons RA (2009) Neonatal exendin-4 treatment reduces oxidative stress and prevents hepatic insulin resistance in intrauterine growth-retarded rats. Am J Physiol Regul Integr Comp Physiol 297: R1785-R1794.

Rahman SA, Nessa A, and Hussain K (2015) Molecular mechanisms of congenital hyperinsulinism. J Mol Endocrinol 54:R119-R129.

Rajan S, Dickson LM, Mathew E, Orr CM, Ellenbroek JH, Philipson LH, and Wicksteed B (2015) Chronic hyperglycemia downregulates GLP-1 receptor signaling in pancreatic $\beta$-cells via protein kinase A. Mol Metab 4:265-276.

Rajan S, Torres J, Thompson MS, and Philipson LH (2012) SUMO downregulates GLP-1-stimulated cAMP generation and insulin secretion. Am J Physiol Endocrinol Metab 302:E714-E723.

Read N, French S, and Cunningham K (1994) The role of the gut in regulating food intake in man. Nutr Rev 52:1-10.

Redondo A, Trigo MV, Acitores A, Valverde I, and Villanueva-Peñacarrillo ML (2003) Cell signalling of the GLP-1 action in rat liver. Mol Cell Endocrinol 204:43-50.

Reiner T, Kohler RH, Liew CW, Hill JA, Gaglia J, Kulkarni RN, and Weissleder R (2010) Near-infrared fluorescent probe for imaging of pancreatic $\beta$ cells. Bioconjug Chem 21:1362-1368.

Renström E, Eliasson L, and Rorsman P (1997) Protein kinase A-dependent and -independent stimulation of exocytosis by cAMP in mouse pancreatic B-cells. $J$ Physiol 502:105-118.

Retterst $\varnothing$ K K (2009) Taspoglutide: a long acting human glucagon-like polypeptide-1 analogue. Expert Opin Investig Drugs 18:1405-1411.

Rhodes CJ and White MF (2002) Molecular insights into insulin action and secretion. Eur J Clin Invest 32 (Suppl 3):3-13.

Roberts DJ, Vertongen P, and Waelbroeck M (2011) Analysis of the glucagon receptor first extracellular loop by the substituted cysteine accessibility method. Peptides 32:1593-1599.

Roed SN, Nøhr AC, Wismann P, Iversen H, Bräuner-Osborne H, Knudsen SM, and Waldhoer M (2015) Functional consequences of glucagon-like peptide-1 receptor cross-talk and trafficking. J Biol Chem 290:1233-1243.

Roed SN, Wismann P, Underwood CR, Kulahin N, Iversen H, Cappelen KA, Schäffer L, Lehtonen J, Hecksher-Soerensen J, Secher A, et al. (2014) Real-time trafficking and signaling of the glucagon-like peptide-1 receptor. Mol Cell Endocrinol 382 938-949.

Rolin B, Larsen MO, Gotfredsen CF, Deacon CF, Carr RD, Wilken M, and Knudsen LB (2002) The long-acting GLP-1 derivative NN2211 ameliorates glycemia and increases beta-cell mass in diabetic mice. Am J Physiol Endocrinol Metab 283: E745-E752.

Rosenstock J, Hanefeld M, Shamanna P, Min KW, Boka G, Miossec P, Zhou T, Muehlen-Bartmer I, and Ratner RE (2014) Beneficial effects of once-daily lixisenatide on overall and postprandial glycemic levels without significant excess of hypoglycemia in type 2 diabetes inadequately controlled on a sulfonylurea with or without metformin (GetGoal-S). J Diabetes Complications 28:386-392.

Rosol TJ (2013) On-target effects of GLP-1 receptor agonists on thyroid C-cells in rats and mice. Toxicol Pathol 41:303-309.

Ruiz-Grande C, Alarcón C, Alcántara A, Castilla C, López Novoa JM, VillanuevaPeñacarrillo ML, and Valverde I (1993) Renal catabolism of truncated glucagonlike peptide 1. Horm Metab Res 25:612-616.

Runge S, Gram C, Brauner-Osborne H, Madsen K, Knudsen LB, and Wulff BS (2003a) Three distinct epitopes on the extracellular face of the glucagon receptor determine specificity for the glucagon amino terminus. J Biol Chem 278:28005-28010

Runge S, Schimmer S, Oschmann J, Schiødt CB, Knudsen SM, Jeppesen CB, Madsen K, Lau J, Thøgersen H, and Rudolph R (2007) Differential structural properties of GLP-1 and exendin-4 determine their relative affinity for the GLP-1 receptor N-terminal extracellular domain. Biochemistry 46:5830-5840.

Runge S, Thøgersen H, Madsen K, Lau J, and Rudolph R (2008) Crystal structure of the ligand-bound glucagon-like peptide-1 receptor extracellular domain. $J$ Biol Chem 283:11340-11347.

Runge S, Wulff BS, Madsen K, Bräuner-Osborne H, and Knudsen LB (2003b) Different domains of the glucagon and glucagon-like peptide-1 receptors provide the critical determinants of ligand selectivity. Br J Pharmacol 138:787-794.
Rutz C, Renner A, Alken M, Schulz K, Beyermann M, Wiesner B, Rosenthal W, and Schülein $\mathrm{R}$ (2006) The corticotropin-releasing factor receptor type 2a contains an N-terminal pseudo signal peptide. J Biol Chem 281:24910-24921.

Salehi M, Gastaldelli A, and D'Alessio DA (2014) Blockade of glucagon-like peptide 1 receptor corrects postprandial hypoglycemia after gastric bypass. Gastroenter ology 146:669-680.e2

Salehi M, Prigeon RL, and D'Alessio DA (2011) Gastric bypass surgery enhance glucagon-like peptide 1-stimulated postprandial insulin secretion in humans. $D i$ abetes 60:2308-2314.

Sancho V, Nuche B, Arnés L, Cancelas J, González N, Díaz-Miguel M, Martín-Duce A, Valverde I, and Villanueva-Peñacarrillo ML (2007) The action of GLP-1 and exendins upon glucose transport in normal human adipocytes, and on kinase activity as compared to morbidly obese patients. Int J Mol Med 19:961-966.

Sarrauste de Menthière C, Chavanieu A, Grassy G, Dalle S, Salazar G, Kervran A Pfeiffer B, Renard P, Delagrange P, Manechez D, et al. (2004) Structural requirements of the N-terminal region of GLP-1-[7-37]-NH2 for receptor interaction and cAMP production. Eur J Med Chem 39:473-480.

Sarson DL, Wood SM, Kansal PC, and Bloom SR (1984) Glucose-dependent insulinotropic polypeptide augmentation of insulin: physiology or pharmacology? $\mathrm{Di}$ abetes 33:389-393.

Satoh F, Beak SA, Small CJ, Falzon M, Ghatei MA, Bloom SR, and Smith DM (2000) Characterization of human and rat glucagon-like peptide-1 receptors in the neurointermediate lobe: lack of coupling to either stimulation or inhibition of adenylyl cyclase. Endocrinology 141:1301-1309.

Saudek F, Brogren CH, and Manohar S (2008) Imaging the beta-cell mass: why and how. Rev Diabet Stud 5:6-12.

Schelshorn D, Joly F, Mutel S, Hampe C, Breton B, Mutel V, and Lütjens R (2012) Lateral allosterism in the glucagon receptor family: glucagon-like peptide 1 induces G-protein-coupled receptor heteromer formation. Mol Pharmacol $\mathbf{8 1}$ 309-318.

Schiöth HB, Lagerström MC, Watanobe H, Jonsson L, Vergoni AV, Ringholm A Skarphedinsson JO, Skuladottir GV, Klovins J, and Fredriksson R (2003) Functional role, structure, and evolution of the melanocortin-4 receptor. Ann N Y Acad Sci 994:74-83.

Schirra J, Nicolaus M, Roggel R, Katschinski M, Storr M, Woerle HJ, and Göke B (2006) Endogenous glucagon-like peptide 1 controls endocrine pancreatic secretion and antro-pyloro-duodenal motility in humans. Gut 55:243-251.

Schirra J, Sturm K, Leicht P, Arnold R, Göke B, and Katschinski M (1998) Exendin(939)amide is an antagonist of glucagon-like peptide-1(7-36)amide in humans. J Clin Invest 101:1421-1430.

Schmidt WE, Siegel EG, and Creutzfeldt W (1985) Glucagon-like peptide-1 but not glucagon-like peptide-2 stimulates insulin release from isolated rat pancreatic islets. Diabetologia 28:704-707.

Schneider G and Fechner U (2004) Advances in the prediction of protein targeting signals. Proteomics 4:1571-1580.

Schneider S (2008) Efforts to develop methods for in vivo evaluation of the native $\beta$-cell mass. Diabetes Obes Metab 10 (Suppl 4):109-118.

Schröder-Tittmann K, Bosse-Doenecke E, Reedtz-Runge S, Ihling C, Sinz A Tittmann K, and Rudolph R (2010) Recombinant expression, in vitro refolding, and biophysical characterization of the human glucagon-like peptide-1 receptor. Biochemistry 49:7956-7965

Schülein R, Westendorf C, Krause G, and Rosenthal W (2012) Functional significance of cleavable signal peptides of G protein-coupled receptors. Eur J Cell Biol 91: 294-299.

Scrocchi LA, Brown TJ, MaClusky N, Brubaker PL, Auerbach AB, Joyner AL, and Drucker DJ (1996) Glucose intolerance but normal satiety in mice with a null mutation in the glucagon-like peptide 1 receptor gene. Nat Med 2:1254-1258.

Segre GV and Goldring SR (1993) Receptors for secretin, calcitonin, parathyroid hormone $(\mathrm{PTH}) / \mathrm{PTH}-$ related peptide, vasoactive intestinal peptide, glucagonlike peptide 1, growth hormone-releasing hormone, and glucagon belong to a newly discovered G-protein-linked receptor family. Trends Endocrinol Metab 4:309-314.

Serre V, Dolci W, Schaerer E, Scrocchi L, Drucker D, Efrat S, and Thorens B (1998) Exendin-(9-39) is an inverse agonist of the murine glucagon-like peptide-1 receptor: implications for basal intracellular cyclic adenosine $3^{\prime}, 5^{\prime}$-monophosphate levels and beta-cell glucose competence. Endocrinology 139:4448-4454.

Service GJ, Thompson GB, Service FJ, Andrews JC, Collazo-Clavell ML, and Lloyd RV (2005) Hyperinsulinemic hypoglycemia with nesidioblastosis after gastricbypass surgery. $N$ Engl J Med 353:249-254.

Shah P, Vella A, Basu A, Basu R, Schwenk WF, and Rizza RA (2000) Lack of suppression of glucagon contributes to postprandial hyperglycemia in subjects with type 2 diabetes mellitus. $J$ Clin Endocrinol Metab 85:4053-4059.

Shan SO and Walter P (2005) Co-translational protein targeting by the signal recognition particle. FEBS Lett 579:921-926.

Shibasaki T, Takahashi H, Miki T, Sunaga Y, Matsumura K, Yamanaka M, Zhang C, Tamamoto A, Satoh T, Miyazaki J, et al. (2007) Essential role of Epac2/Rap1 signaling in regulation of insulin granule dynamics by cAMP. Proc Natl Acad Sci USA 104:19333-19338.

Shields D, Warren TG, Roth SE, and Brenner MJ (1981) Cell-free synthesis and processing of multiple precursors to glucagon. Nature 289:511-514.

Shiue CY, Schmitz A, Schirrmacher R, Shiue GG, and Alavi A (2004) Potential approaches for beta cell imaging with PET and SPECT. Curr Med Chem 4:271-280. Shonberg J, Lopez L, Scammells PJ, Christopoulos A, Capuano B, and Lane JR (2014) Biased agonism at $\mathrm{G}$ protein-coupled receptors: the promise and the challenges-a medicinal chemistry perspective. Med Res Rev 34:1286-1330.

Siu FY, He M, de Graaf C, Han GW, Yang D, Zhang Z, Zhou C, Xu Q, Wacker D, Joseph JS, et al. (2013) Structure of the human glucagon class B G-protein-coupled receptor. Nature 499:444-449.

Skoglund G, Hussain MA, and Holz GG (2000) Glucagon-like peptide 1 stimulates insulin gene promoter activity by protein kinase A-independent activation of the rat insulin I gene cAMP response element. Diabetes 49:1156-1164. 
Sloop KW, Willard FS, Brenner MB, Ficorilli J, Valasek K, Showalter AD, Farb TB, Cao JX, Cox AL, Michael MD, et al. (2010) Novel small molecule glucagon-like peptide-1 receptor agonist stimulates insulin secretion in rodents and from human islets. Diabetes 59:3099-3107.

Smale ST (2010) Luciferase assay. Cold Spring Harb Protoc 2010:pdb.prot5421.

Song WJ, Seshadri M, Ashraf U, Mdluli T, Mondal P, Keil M, Azevedo M, Kirschner LS, Stratakis CA, and Hussain MA (2011) Snapin mediates incretin action and augments glucose-dependent insulin secretion. Cell Metab 13:308-319.

Sonoda N, Imamura T, Yoshizaki T, Babendure JL, Lu JC, and Olefsky JM (2008) Beta-arrestin-1 mediates glucagon-like peptide-1 signaling to insulin secretion in cultured pancreatic beta cells. Proc Natl Acad Sci USA 105:6614-6619.

Souza F, Freeby M, Hultman K, Simpson N, Herron A, Witkowsky P, Liu E, Maffei A, and Harris PE (2006) Current progress in non-invasive imaging of beta cell mass of the endocrine pancreas. Curr Med Chem 13:2761-2773.

St Onge EL and Miller SA (2010) Albiglutide: a new GLP-1 analog for the treatment of type 2 diabetes. Expert Opin Biol Ther 10:801-806.

Steensgaard DB, Thomsen JK, Olsen HB, and Knudsen LB (2008) The molecular basis for the delayed absorption of the once-daily human GLP-1 analoge, liraglutide. Diabetes 57 (Suppl 1):A164.

Stumvoll M, Fritsche A, and Häring HU (2002) Clinical characterization of insulin secretion as the basis for genetic analyses. Diabetes 51 (Suppl 1):S122-S129.

Su H, He M, Li H, Liu Q, Wang J, Wang Y, Gao W, Zhou L, Liao J, Young AA, et al. (2008) Boc5, a non-peptidic glucagon-like peptide-1 receptor agonist, invokes sustained glycemic control and weight loss in diabetic mice. PLoS One 3:e2892.

Suarez-Pinzon WL, Power RF, Yan Y, Wasserfall C, Atkinson M, and Rabinovitch A (2008) Combination therapy with glucagon-like peptide-1 and gastrin restores normoglycemia in diabetic NOD mice. Diabetes 57:3281-3288.

Sun F, Wu S, Wang J, Guo S, Chai S, Yang Z, Li L, Zhang Y, Ji L, and Zhan S (2015) Effect of glucagon-like peptide-1 receptor agonists on lipid profiles among type 2 diabetes: a systematic review and network meta-analysis. Clin Ther 37:225-241.e8.

Svegliati-Baroni G, Saccomanno S, Rychlicki C, Agostinelli L, De Minicis S, Candelaresi C, Faraci G, Pacetti D, Vivarelli M, Nicolini D, et al. (2011) Glucagonlike peptide-1 receptor activation stimulates hepatic lipid oxidation and restores hepatic signalling alteration induced by a high-fat diet in nonalcoholic steatohepatitis. Liver Int 31:1285-1297.

Syme CA, Zhang L, and Bisello A (2006) Caveolin-1 regulates cellular trafficking and function of the glucagon-like peptide 1 receptor. Mol Endocrinol 20:3400-3411.

Szayna M, Doyle ME, Betkey JA, Holloway HW, Spencer RG, Greig NH, and Egan JM (2000) Exendin-4 decelerates food intake, weight gain, and fat deposition in Zucker rats. Endocrinology 141:1936-1941.

Takahashi M, Yamada T, Tooyama I, Moroo I, Kimura H, Yamamoto T, and Okada H (1996) Insulin receptor mRNA in the substantia nigra in Parkinson's disease. Neurosci Lett 204:201-204.

Takhar S, Gyomorey S, Su RC, Mathi SK, Li X, and Wheeler MB (1996) The third cytoplasmic domain of the GLP-1[7-36 amide] receptor is required for coupling to the adenylyl cyclase system. Endocrinology 137:2175-2178.

Tang-Christensen M, Larsen PJ, Göke R, Fink-Jensen A, Jessop DS, Møller M, and Sheikh SP (1996) Central administration of GLP-1-(7-36) amide inhibits food and water intake in rats. Am J Physiol 271:R848-R856.

Tang-Christensen M, Vrang N, and Larsen PJ (1998) Glucagon-like peptide 1(7-36) amide's central inhibition of feeding and peripheral inhibition of drinking are abolished by neonatal monosodium glutamate treatment. Diabetes 47:530-537.

Teng M, Johnson MD, Thomas C, Kiel D, Lakis JN, Kercher T, Aytes S, Kostrowicki J, Bhumralkar D, Truesdale L, et al. (2007) Small molecule ago-allosteric modulators of the human glucagon-like peptide-1 (hGLP-1) receptor. Bioorg Med Chem Lett 17:5472-5478.

Teramoto S, Miyamoto N, Yatomi K, Tanaka Y, Oishi H, Arai H, Hattori N, and Urabe T (2011) Exendin-4, a glucagon-like peptide-1 receptor agonist, provides neuroprotection in mice transient focal cerebral ischemia. J Cereb Blood Flow Metab 31:1696-1705.

Thompson A and Kanamarlapudi V (2014) The regions within the N-terminus critical for human glucagon like peptide-1 receptor (hGLP-1R) cell surface expression. Sci Rep 4:7410.

Thompson A and Kanamarlapudi V (2015) Agonist-induced internalisation of the glucagon-like peptide-1 receptor is mediated by the Goq pathway. Biochem Pharmacol 93:72-84.

Thorens B (1992) Expression cloning of the pancreatic beta cell receptor for the glucoincretin hormone glucagon-like peptide 1. Proc Natl Acad Sci USA 89:8641-8645.

Thorens B, Porret A, Bühler L, Deng SP, Morel P, and Widmann C (1993) Cloning and functional expression of the human islet GLP-1 receptor: demonstration that exendin- 4 is an agonist and exendin-(9-39) an antagonist of the receptor. Diabetes 42:1678-1682.

Thornton K and Gorenstein DG (1994) Structure of glucagon-like peptide (7-36) amide in a dodecylphosphocholine micelle as determined by 2D NMR. Biochemistry 33:3532-3539.

Tibaduiza EC, Chen C, and Beinborn M (2001) A small molecule ligand of the glucagon-like peptide 1 receptor targets its amino-terminal hormone binding domain. J Biol Chem 276:37787-37793.

Toft-Nielsen MB, Damholt MB, Madsbad S, Hilsted LM, Hughes TE, Michelsen BK, and Holst JJ (2001) Determinants of the impaired secretion of glucagon-like peptide-1 in type 2 diabetic patients. J Clin Endocrinol Metab 86:3717-3723.

Toft-Nielson M, Madsbad S, and Holst JJ (1996) The effect of glucagon-like peptide I (GLP-I) on glucose elimination in healthy subjects depends on the pancreatic glucoregulatory hormones. Diabetes 45:552-556.

Toft-Nielsen MB, Madsbad S, and Holst JJ (1999) Continuous subcutaneous infusion of glucagon-like peptide 1 lowers plasma glucose and reduces appetite in type 2 diabetic patients. Diabetes Care 22:1137-1143.

Tokuyama Y, Matsui K, Egashira T, Nozaki O, Ishizuka T, and Kanatsuka A (2004) Five missense mutations in glucagon-like peptide 1 receptor gene in Japanese population. Diabetes Res Clin Pract 66:63-69.
Tomkin GH (2009) Albiglutide, an albumin-based fusion of glucagon-like peptide 1 for the potential treatment of type 2 diabetes. Curr Opin Mol Ther 11:579-588. Tornehave D, Kristensen P, Rømer J, Knudsen LB, and Heller RS (2008) Expression of the GLP-1 receptor in mouse, rat, and human pancreas. J Histochem Cytochem 56:841-851.

Tsuboi T, da Silva Xavier G, Holz GG, Jouaville LS, Thomas AP, and Rutter GA (2003) Glucagon-like peptide-1 mobilizes intracellular $\mathrm{Ca} 2+$ and stimulates mitochondrial ATP synthesis in pancreatic MIN6 beta-cells. Biochem $J$ 369: 287-299.

Trujillo JM and Nuffer W (2014) GLP-1 receptor agonists for type 2 diabetes mellitus: recent developments and emerging agents. Pharmacotherapy 34:1174-1186.

Trujillo JM, Nuffer W, and Ellis SL (2015) GLP-1 receptor agonists: a review of headto-head clinical studies. Ther Adv Endocrinol Metab 6:19-28.

Turton MD, O'Shea D, Gunn I, Beak SA, Edwards CM, Meeran K, Choi SJ, Taylor GM, Heath MM, Lambert PD, et al. (1996) A role for glucagon-like peptide-1 in the central regulation of feeding. Nature 379:69-72.

Udelsman R and Zhang Y (2014) The epidemic of thyroid cancer in the United States: the role of endocrinologists and ultrasounds. Thyroid 24:472-479.

Ueberberg S, Meier JJ, Waengler C, Schechinger W, Dietrich JW, Tannapfel A Schmitz I, Schirrmacher R, Köller M, Klein HH, et al. (2009) Generation of novel single-chain antibodies by phage-display technology to direct imaging agents highly selective to pancreatic $\beta$ - or $\alpha$-cells in vivo. Diabetes 58:2324-2334.

Underwood CR, Garibay P, Knudsen LB, Hastrup S, Peters GH, Rudolph R, and Reedtz-Runge S (2010) Crystal structure of glucagon-like peptide-1 in complex with the extracellular domain of the glucagon-like peptide-1 receptor. J Biol Chem 285:723-730.

Underwood CR, Knudsen LB, Garibay PW, Peters GH, and Reedtz-Runge S (2013) Development of a cysteine-deprived and C-terminally truncated GLP-1 receptor. Peptides 49:100-108.

Underwood CR, Knudsen SM, Wulff BS, Brauner-Osborne H, Lau J, Knudsen LB, Peters GH, and Reedtz-Runge S (2011) Transmembrane alpha-helix 2 and 7 are important for small molecule-mediated activation of the GLP-1 receptor. Phar macology 88:340-348.

Unson CG, Gurzenda EM, Iwasa K, and Merrifield RB (1989) Glucagon antagonists: contribution to binding and activity of the amino-terminal sequence $1-5$, position 12, and the putative alpha-helical segment 19-27. J Biol Chem 264:789-794.

Ussher JR and Drucker DJ (2014) Cardiovascular actions of incretin-based therapies. Circ Res 114:1788-1803.

van Genugten RE, Möller-Goede DL, van Raalte DH, and Diamant M (2013) Extrapancreatic effects of incretin-based therapies: potential benefit for cardiovascularrisk management in type 2 diabetes. Diabetes Obes Metab 15:593-606.

Vázquez P, Roncero I, Blázquez E, and Alvarez E (2005) The cytoplasmic domain close to the transmembrane region of the glucagon-like peptide-1 receptor contains sequence elements that regulate agonist-dependent internalisation. $J$ Endocrinol 186:221-231.

Velez M, Peterson EL, Wells K, Swadia T, Sabbah HN, Williams LK, and Lanfear DE (2015) Association of antidiabetic medications targeting the glucagon-like peptide 1 pathway and heart failure events in patients with diabetes. J Card Fail 21:2-8.

Venkatakrishnan AJ, Deupi X, Lebon G, Tate CG, Schertler GF, and Babu MM (2013) Molecular signatures of G-protein-coupled receptors. Nature 494:185-194.

Verdich C, Flint A, Gutzwiller JP, Näslund E, Beglinger C, Hellström PM, Long SJ, Morgan LM, Holst JJ, and Astrup A (2001) A meta-analysis of the effect of glucagon-like peptide-1 (7-36) amide on ad libitum energy intake in humans. J Clin Endocrinol Metab 86:4382-4389.

Vigneri P, Frasca F, Sciacca L, Pandini G, and Vigneri R (2009) Diabetes and cancer. Endocr Relat Cancer 16:1103-1123.

Vila Petroff MG, Egan JM, Wang X, and Sollott SJ (2001) Glucagon-like peptide-1 increases cAMP but fails to augment contraction in adult rat cardiac myocytes. Circ Res 89:445-452.

Vilsbøll T, Agers $\emptyset$ H, Krarup T, and Holst JJ (2003) Similar elimination rates of glucagon-like peptide- 1 in obese type 2 diabetic patients and healthy subjects. $J$ Clin Endocrinol Metab 88:220-224.

Vilsbøll T and Holst JJ (2004) Incretins, insulin secretion and type 2 diabetes mellitus. Diabetologia 47:357-366.

Vodovozova EL (2007) Photoaffinity labeling and its application in structural biology. Biochemistry 72:1-20.

Vyas AK, Yang KC, Woo D, Tzekov A, Kovacs A, Jay PY, and Hruz PW (2011) Exenatide improves glucose homeostasis and prolongs survival in a murine model of dilated cardiomyopathy. PLoS One 6:e17178.

Wallin E and von Heijne G (1995) Properties of N-terminal tails in G-protein coupled receptors: a statistical study. Protein Eng 8:693-698.

Wang X, Cahill CM, Piñeyro MA, Zhou J, Doyle ME, and Egan JM (1999) Glucagonlike peptide-1 regulates the beta cell transcription factor, PDX-1, in insulinoma cells. Endocrinology 140:4904-4907.

Wang Y, Lomakin A, Kanai S, Alex R, and Benedek GB (2015) Transformation of oligomers of lipidated peptide induced by change in pH. Mol Pharm 12:411-419.

Wang X, Zhou J, Doyle ME, and Egan JM (2001) Glucagon-like peptide-1 causes pancreatic duodenal homeobox-1 protein translocation from the cytoplasm to the nucleus of pancreatic beta-cells by a cyclic adenosine monophosphate/protein kinase A-dependent mechanism. Endocrinology 142:1820-1827.

Watanabe Y, Kawai K, Ohashi S, Yokota C, Suzuki S, and Yamashita K (1994) Structure-activity relationships of glucagon-like peptide-1(7-36)amide: insulinotropic activities in perfused rat pancreases, and receptor binding and cyclic AMP production in RINm5F cells. $J$ Endocrinol 140:45-52.

Wei Y and Mojsov S (1995) Tissue-specific expression of the human receptor for glucagon-like peptide-I: brain, heart and pancreatic forms have the same deduced amino acid sequences. FEBS Lett 358:219-224.

Werner U, Haschke G, Herling AW, and Kramer W (2010) Pharmacological profile of lixisenatide: a new GLP-1 receptor agonist for the treatment of type 2 diabetes. Regul Pept 164:58-64. 
Weston C, Poyner D, Patel V, Dowell S, and Ladds G (2014) Investigating G protein signalling bias at the glucagon-like peptide-1 receptor in yeast. $\mathrm{Br} J$ Pharmacol 171:3651-3665.

Wettergren A, Maina P, Boesby S, and Holst JJ (1997) Glucagon-like peptide-1 7-36 amide and peptide YY have additive inhibitory effect on gastric acid secretion in man. Scand J Gastroenterol 32:552-555.

Wettergren A, Petersen H, Orskov C, Christiansen J, Sheikh SP, and Holst JJ (1994) Glucagon-like peptide-1 7-36 amide and peptide YY from the L-cell of the ileal mucosa are potent inhibitors of vagally induced gastric acid secretion in man Scand J Gastroenterol 29:501-505.

Wettergren A, Pridal L, Wøjdemann M, and Holst JJ (1998) Amidated and nonamidated glucagon-like peptide-1 (GLP-1): non-pancreatic effects (cephalic phase acid secretion) and stability in plasma in humans. Regul Pept 77:83-87.

Wettergren A, Schjoldager B, Mortensen PE, Myhre J, Christiansen J, and Holst JJ (1993) Truncated GLP-1 (proglucagon 78-107-amide) inhibits gastric and pancreatic functions in man. Dig Dis Sci 38:665-673.

Wheeler MB, Gelling RW, McIntosh CH, Georgiou J, Brown JC, and Pederson RA (1995) Functional expression of the rat pancreatic islet glucose-dependent insulinotropic polypeptide receptor: ligand binding and intracellular signaling properties. Endocrinology 136:4629-4639.

Wheeler MB, Lu M, Dillon JS, Leng XH, Chen C, and Boyd AE 3rd (1993) Functional expression of the rat glucagon-like peptide-I receptor, evidence for coupling to both adenylyl cyclase and phospholipase-C. Endocrinology 133:57-62.

Wicki A, Wild D, Storch D, Seemayer C, Gotthardt M, Behe M, Kneifel S, Mihatsch MJ, Reubi J-C, Mäcke HR, et al. (2007) [Lys40(Ahx-DTPA-111In)NH2]-Exendin-4 is a highly efficient radiotherapeutic for glucagon-like peptide-1 receptor-targeted therapy for insulinoma. Clin Cancer Res 13:3696-3705.

Widmann C, Bürki E, Dolci W, and Thorens B (1994) Signal transduction by the cloned glucagon-like peptide-1 receptor: comparison with signaling by the endogenous receptors of beta cell lines. Mol Pharmacol 45:1029-1035.

Widmann C, Dolci W, and Thorens B (1995) Agonist-induced internalization and recycling of the glucagon-like peptide- 1 receptor in transfected fibroblasts and in insulinomas. Biochem $J \mathbf{3 1 0} \cdot 203-214$.

Widmann C, Dolci W, and Thorens B (1996a) Heterologous desensitization of the glucagon-like peptide-1 receptor by phorbol esters requires phosphorylation of the cytoplasmic tail at four different sites. J Biol Chem 271:19957-19963.

Widmann C, Dolci W, and Thorens B (1996b) Desensitization and phosphorylation of the glucagon-like peptide-1 (GLP-1) receptor by GLP-1 and 4-phorbol 12-myristate 13-acetate. Mol Endocrinol 10:62-75.

Widmann C, Dolci W, and Thorens B (1997) Internalization and homologous desensitization of the GLP-1 receptor depend on phosphorylation of the receptor carboxyl tail at the same three sites. Mol Endocrinol 11:1094-1102.

Wild D, Béhé M, Wicki A, Storch D, Waser B, Gotthardt M, Keil B, Christofori G, Reubi JC, and Mäcke HR (2006) [Lys40(Ahx-DTPA-111In)NH2] exendin-4, a very promising ligand for glucagon-like peptide-1 (GLP-1) receptor targeting. $J$ Nucl Med 47:2025-2033.

Wild D, Wicki A, Mansi R, Béhé M, Keil B, Bernhardt P, Christofori G, Ell PJ, and Mäcke HR (2010) Exendin-4-based radiopharmaceuticals for glucagonlike peptide-1 receptor PET/CT and SPECT/CT. J Nucl Med 51:1059-1067.

Wildhage I, Trusheim H, Göke B, and Lankat-Buttgereit B (1999) Gene expression of the human glucagon-like peptide-1 receptor is regulated by Sp1 and Sp3. Endocrinology 140:624-631.

Willard FS, Bueno AB, and Sloop KW (2012a) Small molecule drug discovery at the glucagon-like peptide-1 receptor. Exp Diabetes Res 2012:709893.

Willard FS, Wootten D, Showalter AD, Savage EE, Ficorilli J, Farb TB, Bokvist K Alsina-Fernandez J, Furness SG, Christopoulos A, et al. (2012b) Small molecule allosteric modulation of the glucagon-like peptide-1 receptor enhances the insulinotropic effect of oxyntomodulin. Mol Pharmacol 82:1066-1073.

Wilmen A, Göke B, and Göke R (1996) The isolated N-terminal extracellular domain of the glucagon-like peptide-1 (GLP)-1 receptor has intrinsic binding activity. FEBS Lett 398:43-47.

Wilmen A, Van Eyll B, Göke B, and Göke R (1997) Five out of six tryptophan residues in the N-terminal extracellular domain of the rat GLP-1 receptor are essential for its ability to bind GLP-1. Peptides 18:301-305.

Wilson BE, Mochon E, and Boxer LM (1996) Induction of bcl-2 expression by phosphorylated CREB proteins during B-cell activation and rescue from apoptosis. $M o l$ Cell Biol 16:5546-5556.

Wiseman T, Williston S, Brandts JF, and Lin LN (1989) Rapid measurement of binding constants and heats of binding using a new titration calorimeter. Anal Biochem 179:131-137.

Wootten D, Christopoulos A, and Sexton PM (2013a) Emerging paradigms in GPCR allostery: implications for drug discovery. Nat Rev Drug Discov 12:630-644.

Wootten D, Savage EE, Valant C, May LT, Sloop KW, Ficorilli J, Showalter AD, Willard FS, Christopoulos A, and Sexton PM (2012) Allosteric modulation of endogenous metabolites as an avenue for drug discovery. Mol Pharmacol 82:281-290.

Wootten D, Savage EE, Willard FS, Bueno AB, Sloop KW, Christopoulos A, and Sexton PM (2013b) Differential activation and modulation of the glucagon-like peptide-1 receptor by small molecule ligands. Mol Pharmacol 83:822-834.

Wootten D, Simms J, Koole C, Woodman OL, Summers RJ, Christopoulos A and Sexton PM (2011) Modulation of the glucagon-like peptide-1 receptor signaling by naturally occurring and synthetic flavonoids. J Pharmacol Exp Ther 336: $540-550$.

Wootten D, Simms J, Miller LJ, Christopoulos A, and Sexton PM (2013c) Polar transmembrane interactions drive formation of ligand-specific and signal pathwaybiased family B G protein-coupled receptor conformations. Proc Natl Acad Sci USA 110:5211-5216.

Wootten D, Reynolds CA, Koole C, Smith KJ, Mobarec JC, Simms J, Quon T, Coudrat T, Furness SG, Miller LJ, et al. (2016) A hydrogen-bonded polar network in the core of the glucagon-like peptide-1 receptor is a fulcrum for biased agonism: lessons from class B crystal structures. Mol Pharmacol 89:335-347.

Wu Z, Todorov I, Li L, Bading JR, Li Z, Nair I, Ishiyama K, Colcher D, Conti PE, Fraser SE, et al. (2011) In vivo imaging of transplanted islets with 64Cu-DO3A-VSCys40-Exendin-4 by targeting GLP-1 receptor. Bioconjug Chem 22:1587-1594.

Xiao Q, Giguere J, Parisien M, Jeng W, St-Pierre SA, Brubaker PL, and Wheeler MB (2001) Biological activities of glucagon-like peptide-1 analogues in vitro and in vivo. Biochemistry 40:2860-2869.

Xiao Q, Jeng W, and Wheeler MB (2000) Characterization of glucagon-like peptide-1 receptor-binding determinants. J Mol Endocrinol 25:321-335.

Xiong Y, Guo J, Candelore MR, Liang R, Miller C, Dallas-Yang Q, Jiang G, McCann PE, Qureshi SA, Tong X, et al. (2012) Discovery of a novel glucagon receptor an tagonist N-[(4-(1S)-1-[3-(3, 5-dichlorophenyl)-5-(6-methoxynaphthalen-2-yl)-1Hpyrazol-1-yl]ethylphenyl)carbonyl]- $\beta$-alanine (MK-0893) for the treatment of type II diabetes. J Med Chem 55:6137-6148.

Xu G, Kaneto H, Lopez-Avalos MD, Weir GC, and Bonner-Weir S (2006) GLP-1/ exendin- 4 facilitates $\beta$-cell neogenesis in rat and human pancreatic ducts. Diabetes Res Clin Pract 73:107-110.

Yachida S, Jones S, Bozic I, Antal T, Leary R, Fu B, Kamiyama M, Hruban RH, Eshleman JR, Nowak MA, et al. (2010) Distant metastasis occurs late during the genetic evolution of pancreatic cancer. Nature 467:1114-1117.

Yamada C, Yamada Y, Tsukiyama K, Yamada K, Udagawa N, Takahashi N, Tanaka K, Drucker DJ, Seino Y, and Inagaki N (2008) The murine glucagon-like peptide-1 receptor is essential for control of bone resorption. Endocrinology 149:574-579.

Yang D, de Graaf C, Yang L, Song G, Dai A, Cai X, Feng Y, Reedtz-Runge S, Hanson MA, Yang H, et al. (2016) Structural determinants of binding the seventransmembrane domain of the glucagon-like peptide-1 receptor. $J$ Biol Chem 291:12991-13004

Yang L, Yang D, de Graaf C, Moeller A, West GM, Dharmarajan V, Wang C, Siu FY, Song G, Reedtz-Runge S, et al. (2015b) Conformational states of the full-length glucagon receptor. Nat Commun 6:7859.

Yang DH, Zhou CH, Liu Q, and Wang MW (2015a) Landmark studies on the glucagon subfamily of GPCRs: from small molecule modulators to a crystal structure. Acto Pharmacol Sin 36:1033-1042.

Yaqub T, Tikhonova IG, Lättig J, Magnan R, Laval M, Escrieut C, Boulègue C, Hewage C, and Fourmy D (2010) Identification of determinants of glucosedependent insulinotropic polypeptide receptor that interact with $\mathrm{N}$-terminal biologically active region of the natural ligand. Mol Pharmacol 77:547-558.

Young AA, Gedulin BR, Bhavsar S, Bodkin N, Jodka C, Hansen B, and Denaro M (1999) Glucose-lowering and insulin-sensitizing actions of exendin-4: studies in obese diabetic (ob/ob, db/db) mice, diabetic fatty Zucker rats, and diabetic rhesus monkeys (Macaca mulatta). Diabetes 48:1026-1034.

Yusta B, Baggio LL, Estall JL, Koehler JA, Holland DP, Li H, Pipeleers D, Ling Z, and Drucker DJ (2006) GLP-1 receptor activation improves beta cell function and urvival following induction of endoplasmic reticulum stress. Cell Metab 4: 391-406.

Zaccardi F, Htike ZZ, Webb DR, Khunti K, and Davies MJ (2016) Benefits and harms of once-weekly glucagon-like peptide-1 receptor agonist treatments: a systematic review and network meta-analysis. Ann Intern Med 164:102-113.

Zander M, Madsbad S, Madsen JL, and Holst JJ (2002) Effect of 6-week course of glucagon-like peptide 1 on glycaemic control, insulin sensitivity, and beta-cell function in type 2 diabetes: a parallel-group study. Lancet 359:824-830.

Zheng H, Cai L, and Rinaman L (2015) Distribution of glucagon-like peptide 1-immunopositive neurons in human caudal medulla. Brain Struct Funct $\mathbf{2 2 0}$ 1213-1219

Zhao T, Parikh P, Bhashyam S, Bolukoglu H, Poornima I, Shen YT, and Shannon RP (2006) Direct effects of glucagon-like peptide-1 on myocardial contractility and glucose uptake in normal and postischemic isolated rat hearts. J Pharmacol Exp Ther 317:1106-1113.

Zhao H, Wei R, Wang L, Tian Q, Tao M, Ke J, Liu Y, Hou W, Zhang L, Yang J, et al (2014) Activation of glucagon-like peptide-1 receptor inhibits growth and promotes apoptosis of human pancreatic cancer cells in a cAMP-dependent manner. Am $J$ Physiol Endocrinol Metab 306:E1431-E1441.

Zhou FX, Cocco MJ, Russ WP, Brunger AT, and Engelman DM (2000) Interhelical hydrogen bonding drives strong interactions in membrane proteins. Nat Struct Biol 7:154-160.

Zunz E and LaBarre J (1928) Hyperinsulinémie consécutive a l'injection de solution de secrétine non hypotensive. C R Soc Biol 98:1435-1438.

Zwier JM, Roux T, Cottet M, Durroux T, Douzon S, Bdioui S, Gregor N, Bourrier E Oueslati N, Nicolas L, et al. (2010) A fluorescent ligand-binding alternative using Tag-lite® technology. J Biomol Screen 15:1248-1259. 